ISTITUTO LOMBARDO

ACCADEMIA di SCIENZE E LETTERE

INCONTRO DI STUDI N. 68

\title{
IDEE IN CERCA DI PAROLE \\ PAROLE IN CERCA DI IDEE
}

20 dicembre 2012

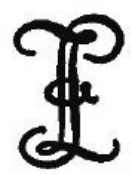

MILANO

2014 
Comitato di Presidenza

Gianpiero Sironi Presidente

Angelo Stella Vicepresidente

Giuseppe Frasso Segretario della Classe di Lettere

Leone Corradi dell'Acqua Segretario della Classe di Scienze

Cancelliere

Adele Bianchi Robbiati 
Al decano dell'Istituto Lombardo Maurizio Vitale nel suo novantesimo compleanno 



\title{
Gianpiero Sironi
}

\author{
«ABitARE LA LINGUA»
}

Sono molto lieto di rivolgere un saluto di benvenuto ai relatori ed a tutti i partecipanti.

L'incontro odierno ha un significato particolare: quello di onorare Maurizio Vitale, decano dell'Istituto, che all'Istituto appartiene da quasi cinquanta anni. Il titolo per il quale lo si onora non è tanto l'appartenere a questa Accademia di Scienze e Lettere da un tempo maggiore di quello di ogni altro membro o socio (o quello di avere una età di tutto rispetto), ma di avere continuato a essere un Maestro nello studio della storia della lingua italiana, la disciplina che ha insegnato per quattro decenni all'Università degli Studi di Milano.

L'«incontro di studio» vede invitati come relatori colleghi e allievi che illustreranno alcuni punti focali della sua lezione, momenti e temi, questioni e figure che hanno stimolato e ancora orientano la sua ricerca.

Vorrei ricordare uno di questi temi, trattato da Maurizio Vitale molto recentemente, lo scorso anno, in un testo presentato all'Accademia dei Lincei, dal titolo La lingua italiana e l'unità nazionale.

Inizia con l'affermazione che la lingua costituisce l'elemento primario della identità nazionale: un'affermazione sempre coinvolgente, in particolare in momenti, come questi, dove è doveroso per la cultura identificare ciò che unisce, non ciò che può dividere.

Mi piace rilevare che una considerazione, anzi un programma unitario, si colloca all'origine del ruolo di protagonista che l'Istituto Lombardo ha avuto nel dibattito sulla lingua della nazione che si voleva costituire, a partire dall'epoca

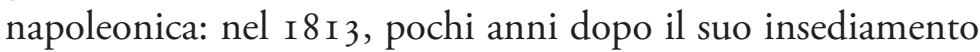


a Milano, l'allora «Istituto Nazionale Italiano di Scienze, Lettere ed Arti» - di cui il Lombardo è continuazione e sviluppo, con nome che lo àncora alla tradizione illuministica e riformatrice della capitale morale - fu indotto «d'improvviso a occuparsi di problemi di lingua da una lettera del Ministro dell'Interno del Regno Italico, il conte Luigi Vaccari».

Da allora, l'impegno del Lombardo per lo studio della lingua italiana è stato costante, nella lezione di Monti, Stratico, Gherardini, Manzoni, Ascoli, Salvioni ed altri ancora, per giungere e proseguire nella riflessione storica e nella proiezione critica di cui Maurizio Vitale è eccellente interprete.

Desidero ringraziare i Colleghi del Comitato Scientifico, che hanno programmato l'incontro: Ilaria Bonomi, Gabriella Cartago, Giuseppe Frasso, Silvia Morgana, Enrico Isacco Rambaldi, Angelo Stella.

Tra gli studiosi che hanno inviato un messaggio di partecipazione, desidero segnalare:

- il sindaco di Milano Giuliano Pisapia;

- Gian Antonio Danieli, presidente dell'Istituto Veneto di Scienze, Lettere ed Arti;

- Fulvio Tessitore, presidente dell'Unione Accademica Nazionale, che assicura la sua ideale partecipazione, con i più vivi complimenti e auguri all'amico;

- Francesco Sabatini, presidente onorario dell'Accademia della Crusca, che rende piena testimonianza del debito istituzionale verso Maurizio Vitale per il suo lavoro instancabile, per il dono dei frutti preziosi delle sue ricerche, per la guida e l'incoraggiamento che ha dato a una folta schiera di seguaci;

- Lamberto Maffei, presidente dell'Accademia dei Lincei, del cui messaggio desidero leggere un passo:

Il socio Vitale, che ho avuto modo di conoscere meglio dopo la mia presidenza, mi ha immediatamente colpito per la sua raffinata sensibilità culturale. Quando lo si incontra lungo le belle sale dei Lincei, ci accoglie sempre con un sorriso cordiale e amichevole: in 
lui il 'continuo lavoro' di studio e di ricerca traspare dalla sensibilità e dalla serenità del volto. Quando tenne la sua conferenza per le celebrazioni dell'Unità d'Italia, citando Elias Canetti, Vitale disse «La cultura è anche legame tra gli individui; non abitiamo un paese, ma una lingua dove sono riposte le nostre tradizioni e la nostra maniera di relazionarci e anche di pensare. Ho imparato da lui e condivido il suo pensiero che la cultura è sorella della gentilezza e dell'amicizia tra le persone e tra i popoli».

Sono sicuro di avere in comune con il professor Vitale l'idea che la cultura è l'unica speranza che abbiamo di uscire da questi momenti, come direbbe il Guicciardini, di barbarie.

L'incontro di studio, promosso dal nostro Istituto, si apre e si svolge con la partecipe collaborazione di:

- Università degli Studi di Milano

- Centro Nazionale Studi Manzoniani

- Centro di Judaica Goren-Goldstein

Desidero ricordare che al termine dell'incontro di studio avrà luogo, in Palazzo Landriani, l'inaugurazione della mostra "Il continuo lavoro di un Maestro», che ripercorre e illustra con un catalogo critico, in una esplorazione sequenziale, dalle Origini al Novecento, il nostro divenire linguisticamente italiani. 

PARTE PRIMA 



\begin{abstract}
Angelo Stella
I LUOGHI DELLA LINGUA, I TEMPI DEGLI SCRITTORI

Premessa a UN PERCORSO BIBLIOGRAFICO
\end{abstract}

1. "Il continuo lavoro di un Maestro» è titolo segnaletico e cornice di questo incontro e della mostra critico-bibliografica, con cui l'Istituto nostro - e prima di Vincenzo Monti, Alessandro Manzoni, Graziadio Isaia Ascoli, Carlo Salvioni - invita e indirizza su un percorso privilegiato di studio che, tappa dopo tappa, ha esplorato la millenaria storia della lingua italiana. L'obbligo ci deriva, per ricordare una minima tra le molte e forti motivazioni, proprio dalla nostra protostoria istituzionale, illustrata da Maurizio Vitale nel saggio esposto, con proposito stimolante, nella sede dell'Accademia della Crusca nell'autunno 1983, su Lombardi e Toscani nella questione del Vocabolario (ora in la Veneranda Favella. Studi di storia della lingua italiana, Napoli, Morano, I988, pp. 487563): è a Milano che nel secondo decennio dell'Ottocento, nel contesto di un rinnovamento culturale voluto dagli uomini del Regno Italico e non accantonato dal governo austriaco, Luigi Lamberti, Giovanni Paradisi, Simone Stratico, Vincenzo Monti, promuovono «la compilazione del vocabolario rivoluzionario", alternativo a quello della Crusca, che si provano ad aggiornare su repertori lessicografici come «'Enciclopedia metodica francese delle arti e dei mestieri, il Dizionario francese delle arti e delle scienze del Lunier, il Dizionario delle arti e dei mestieri di Francesco Griselini e un rilevante numero di lessici speciali italiani e stranieri» (pp. 5 I4-I 5 ).

Posta la cornice, si prospettano, in sintetica successione, le pagine offerte agli studiosi da Vitale nell'arco di oltre sessant'anni, I95 I-20I2, sul «filo unitario» del tracciato di autori e di teorici toscani e non, che si dispongono in capitoli, in 
ordine cronologico, di una opera articolata e organica, che la memoria critica e bibliografica sa collocata negli annali della disciplina che ha guidato la cultura del nostro secondo Novecento, a fianco e in dialogo, per ricordare studiosi esemplari, con Alfredo Schiaffini, Bruno Migliorini, Giovanni Nencioni, Gianfranco Folena, Maria Corti.

L'accosto a queste pagine non può non essere cauto, come lo è stato per molti di noi, negli anni dell'apprendistato universitario: dall'ingresso non frequentato da lui segnalato, premessa alla scoperta di uno spazio affascinante, quel centinaio di pagine, La Lingua volgare della Cancelleria VisconteoSforzesca nel Quattrocento (1953, ma licenziato nel I952, e annunciato nel 1949 da un articolo su «Paideia»): era l'esito a stampa della tesi relata da Antonio Viscardi, con dedica (delle sue dediche il lettore dovrà tenere conto e dei sentimenti che le dettano) «Ai miei genitori». Intorno a questa monografia si erano disposti e progressivamente si disponevano altri contributi storico-linguistici, focalizzati in particolare sul dibattito culturale e grammaticale dell'Umanesimo e del Rinascimento volgari, e sul Purismo di Antonio Cesari, preparatori al programmato volume, evidentemente già mentalmente disegnato, su La Questione della Lingua: apparirà nel I960, a introdurre, in quasi contemporaneità e a latere della Storia della lingua italiana di Migliorini, il millenario dei Placiti cassinesi.

È doveroso, almeno nelle circostanze di questa giornata, segnalare che sono stati $\mathrm{i}$ "Rendiconti» dell'Istituto, a ospitare nel I95 I il contributo su L'Atteggiamento generale di G. F. Fortunio in ordine al problema ortografico, precorritore di un indirizzo di ricerca apprezzato decenni più tardi (ora in Studi di Storia della lingua italiana, Milano, LED, I 992, pp. 95-I Io).

Nella severa griglia dell'indagine sui documenti cancellereschi, nella apparente asetticità della interrogazione grammaticale, nei riscontri delle formule enunciative, si sente la sottesa presenza comparativa dell'altra grammatica, della lingua che 
la storia aveva già collocato a imprescindibile riferimento di ogni scripta, quella della tradizione letteraria fiorentina, la coinè elitaria, la gramatica, che consente di scalare ma anche di comprendere il variare degli usi municipali e regionali nei luoghi, nei tempi, nelle pagine e sulle bocche delle parole. E Vitale sembra preavvisarci, quasi in previsione dell'attenzione che stava per riversarsi sui testi 'popolari', che i suoi studi percorreranno le vette espressive, ma sempre misurando i sentieri e gli angoli della scrittura di un fedele cancelliere o di uno sconosciuto postulante, perché sono testi di lingua anche «le lettere degli uomini d'arme, le petizioni di umili popolani al duca e ai segretari ducali, i verbali degli interrogatori resi da delinquenti comuni o, comunque, da indiziati» (p. I9): è un imperativo dello storico della lingua ascoltare la voce di Manzoni e quella riportata di Gian Giacomo Mora. A volte, anche la loro parola si chiarisce nel registro del sottinteso.

Della via maestra, e di alta quota, erano confermati i passi di avvio e il tracciato, proprio in apertura alla Questione della lingua (p. 5):

Il fiorentino antico e scritto, quale si fissa con procedimenti d'arte nei grandi scrittori trecenteschi, e si impone mediante il prestigio letterario e culturale dei sommi auctores fiorentini e nel solco della prodigiosa fortuna di Firenze borghese e mercantile nell'età comunale, ed è codificato dalla regolamentazione grammaticale cinquecentesca, è il fondamento dell'italiano comune; si può dir meglio che la lingua comune e nazionale italiana è il fiorentino quale è venuto affermandosi e imponendosi attraverso una serie complessa di vicende culturali e sociali in Italia come lingua di tutta la nazione nel corso della nostra storia civile.

Direttrice, al cui altro capo, certo caposaldo inamovibile verso un arrivo sempre spostato in avanti, inscriveva il giudizio, da lui memorizzato e spesso ripetuto con passione incidente, dettato da Carlo Cattaneo, il più tenace oppositore del (non inteso) neofiorentinismo manzoniano, comunque concor- 
de nella difesa della «lingua ... il solo vìncolo della vita e del nome commune ... prezioso patrimonio dei pòveri e dei ricchi, dei dotti e del volgo, la lingua, la lingua, che, più dell'alpi inùtili e del mare non nostro, segna il confine e la divisa della nostra gloriosa nazione».

Non è lecito indagare quanto di rimpianti di nostalgie di invidie possano nutrire molti dei presenti per non avere ascoltato e verso quanti hanno ascoltato le lezioni, lectiones ex cathedra, di Maurizio Vitale, serrate dall'analisi dei dati, maieuticamente gravitanti alla scoperta di un approdo concettuale, distribuite in una architettura sintattica di cui la sua graduata dizione sapeva far comprendere e ammirare l'ordine e le gerarchie; architettura ridisegnata nell'equilibrio delle frasi complesse, dove si risente quella voce sicura graduare analiticamente dati e interpretazioni dei dati, marcando i segnali critici che convogliano a un giudizio, definitivo.

Gli annuari dell'Università degli Studi di Milano ci parlano dei corsi accademici, sempre nuovi nel tema, organici e progressivi nel metodo e nell'approfondimento, che hanno un primo significativo e quasi contemporaneo esito editoriale nelle due raccolte di fatto monografiche, la già citata Veneranda favella del I988, e L'Oro nella lingua. Contributi per una storia del tradizionalismo e del purismo italiano (MilanoNapoli, Riccardo Ricciardi Editore, I986: dedicato «Alla memoria di Riccardo Bacchelli e Raffaele Mattioli», forse nel ricordo delle notti di via Bigli).

Il codificarsi dell'italiano scritto della norma fiorentina, il virare e radicalizzarsi del tradizionalismo nel purismo: la strada maestra è naturalmente quella segnata dalle tre significative edizioni del Vocabolario della Crusca, la prima del i6 I2, la terza del I69I, stampata a Firenze e non a Venezia, la quarta, innovata, degli anni I 729-I 738, per giungere all'ingenuo tentativo restauratore del purismo cesariano cui va riconosciuto il «gran merito», alla vigilia della restaurazione dell'Accademia 
della Crusca voluta da Napoleone, e delle 'proposte' riformatrici di questo Istituto, "d'aver richiamato, in un periodo di forti spinte teoriche centrifughe e di diverse istanze di profondo rinnovamento culturale (con i possibili eccessi anche in fatto di lingua), il concetto di lingua unitaria e naturale e il principio del valore della tradizione pur nell'inarrestabile processo di modificazione linguistica» (p. 524).

Dello specifico «naturalezza», che Cesari monopolizza e si potrebbe postillare, senza offenderlo, brevetta con il recupero dei «valori linguistici antichi», nella misura in cui se ne appropriavano i comici fiorentini, da Leonardo Salviati, a Buonarroti il Giovane con Anton Maria Salvini, «il tradizionalista» più innovatore Salvini, a Lorenzo Lippi con i suoi annotatori, viene rivendicato un possesso primario al parlato popolare, emergenza storica che il nostro studioso deve affrontare e affronta nel saggio Di alcune rivendicazioni secentesche della «eccellenza» dei dialetti (del I980: ora in la Veneranda favella, pp. 305-24). Apodittici i rinvii di teorici minori, come l'anonimo identificatosi con l'ossimorico pseudonimo di Partenio Tosco, che richiamava ai "diritti della natura contro l'arte», o il milanese Biffi, che osava impudentemente sostenere: «el nost lenguag ... al se chonfa tant con la Natura ch'al nol chad sforzal naghot intel droual ... al ghe basta quel sprendò nassù ch'al à con lù inscì pur pur» (ivi, p. 3 I3).

L'attenzione dello storico della lingua stava focalizzandosi su autori che, in diversi tempi e in diversi luoghi, si muovono con «un orientamento intellettuale antipuristico, proprio nell'interesse rivolto al linguaggio orale e ordinario nel suo uso comunicativo e popolare» (ivi, p. 3is). È con la ferma convinzione di una definitiva scelta razionale e appassionata da una sempre rigorosa militanza ideologica, che Vitale legge sul paradigma di una lingua virtuale, quasi la gramatica rispetto ai volgari danteschi, la lingua italiana meno imperfetta, quella appunto della tradizione letteraria, che consente la 
comprensione reciproca tra quante sono grammaticalmente e lessicalmente divergenti, e di queste tutela la varietà continua, mentre ne misura le distanze e le convergenze.

2. Sia consentito richiamare l'ascolto dei presenti su tre monografie meno attese, per aspetti ancora controversi, o per il nome di due autori per diverse cause poco frequentati, da alcuni ostracizzati, in una esposizione colloquiale, che espone a sottotitolo «Invito alla rilettura di Giovanni Boccaccio, di Gian Giorgio Trissino, di Riccardo Bacchelli».

Si farà riferimento a tre delle analisi monografiche, datate rispettivamente 2002, 2010, I999, tra altre, anche più coinvolgenti, che a partire dal chiudersi degli anni Ottanta, sull'abbrivo delle raccolte sopracitate, Vitale ha dedicato (e sta dedicando), in una organica progressione, ai nostri 'grandi' scrittori, Manzoni (I 986 e I 992), Leopardi (I992), Petrarca (1996), Tasso (2007), Ariosto (20 I2), Parini (di prossima uscita: in attesa, sulla soglia del romitorio, Vico).

Autori regolati, non espressionistici, nelle cui pagine dato che si avverte facilmente la mescolanza di elementi discordanti nel pastiche, e meno nel composto, nel graduato, nell'armonico - è appena percepibile il sotteso contrasto e il trascolorare delle parole.

I due volumi del ricco, originale ed esaustivo saggio $\mathrm{Il} \mathrm{Ca}$ polavoro del Boccaccio e due diverse redazioni, vedono il contributo di Vittore Branca, Variazioni narrative e stilistiche (in copertina: stilistiche e narrative), integrarsi e arricchirsi con $L a$ Riscrittura del "Decameron». I mutamenti linguistici, di Maurizio Vitale. Che si rivolge in primo luogo, con la consueta elegante e perciò non dichiarata selettività, ai fedeli dell'antica accademia costituita da Dante Alighieri con il De vulgari eloquentia, alimentata nei secoli dalla parola di grandi scrittori e critici, da Leopardi e Manzoni, a Gadda, Pasolini (capolinea in La Questione della lingua. Nuova edizione, Palermo, Prin- 
cipato, I984, pp. 6I6-2 I), Meneghello, dai Deputati fiorentini alla revisione del capolavoro del Boccaccio ai moderni e recenti suoi esploratori, Pietro Fanfani, Adolfo Mussafia, Aldo Rossi, Francesco Bruni.

Critici e filologi non hanno accolto con una critica concorde la valutazione del manoscritto Parigino It. 482 e la valorizzazione del Berlinese Hamilton 90 compiute da Vittore Branca: con il compianto docente padovano, il capolavoro narrativo del nostro aureo Trecento entrava, come accaduto a Petrarca grazie ai manoscritti vaticani, nella esemplarità della critica degli scartafacci, dentro i suggestivi anditi delle varianti d'autore. Quasi a esporre un segnale di pericolo al confine filologico, Branca parla di «variazioni narrative e stilistiche»; e Vitale richiede, prima di consegnarci un lasciapassare, una documentata attenzione ai «tratti che connotano la grammatica del discorso propria dei codici esaminati» (p. 42). Discorso come espressione formale di un contenuto narrativo, formale perché di valori e di strutture linguistiche semplici e complesse. Non la notomia astratta di una metalingua prospettica, non lo schema di una potenzialità comunicativa, ma la fisiologia di un atto espressivo storicamente compiuto, in funzione di un pubblico contemporaneo: fiorentino, lombardo, apulo, europeo, femminile: Firenze rimane al centro e chissà dove i confini delle aree laterali, e chissà quale e quanta e dove la posterità.

Perché, nello spazio di due decenni, il Boccaccio sottopone a una «larga revisione» il suo capolavoro? Vitale focalizza (ci si precipiti a p. 545, al conclusivo capitolo La «ratio» correttoria nei suoi esiti significativi) i due momenti in «differenti condizioni culturali [...] con diversa e consapevole intenzione d'arte». Formula che, adattata alla fine ricezione di quanti hanno pur ereditato qualche cromosoma spirituale di Cisti fornaio, può essere in parafrasi: il Decameron è realizzato negli anni Cinquanta del Trecento in un tosco-fiorentino nobilitato dal 
selettivo ricorso (come nelle opere minori) al vocabolario 'romanzo' in diffuso dialogo con una coinè letteraria e mercantile sovramunicipale, affidato alla garanzia non solo sintattica del sotteso latino, ora «liviano», destinato alla competenza di un pubblico internazionale (parificando ovviamente - come allora nei fatti e oggi storicamente doveroso - nazione a comune) mediamente o meglio mezzanamente colto.

Negli anni Settanta del Trecento, grazie anche alla congiunta fortuna dell'insieme dell'opera boccacciana e della Commedia, il fiorentino non è più - sempre in àmbito di ricezione - la lingua di un municipio, ma è lingua di una vasta area culturale, ora la grammatica viene compresa, le forme e le formule tecniche realistiche e comiche di un oggi che non è più quello di Dante possono farsi gustare, nell'impasto della scrittura, come varietà, oltre e più che diatopica, espressiva. Così le due opposte operazioni della «azione» autoriale, del togliere o dell'aggiungere colori, magari gli stessi, in luoghi diversi, azione «larga» perché sistematica, risultano interdipendenti e artisticamente convergenti. Da una parte la ripulitura della patina letteraria dovuta alla tradizione novellistica 'europea', «forme e voci genericamente dotte e antiche», dall'altra voci «umili e basse» per la diffusione rastremata di altre «forme e voci divenute ormai comuni e correnti nelle abitudini scritte trecentesche». Un percorso - il riferimento aiuta a comprendere e a presentire l'affascinante difficoltà di una analisi micrometrica, perché entriamo nell'aula dell'edificio critico di Vitale - soprattutto nella pars destruens simile e diverso da quello che completerà Manzoni dal Fermo e dalla Ventisettana alla Quarantana, cancellando dal basso i lombardismi, dall'al to col decrescimento della letterarietà ( $L a$ Lingua di Alessandro Manzoni, Milano, Cisalpino, I992, pp. I8-28).

Rimanendo al Decameron, fissato il tratto di questa linea mediana, nella sua precisa proprietà denotativa e connotativa, diviene possibile, alla competenza interpretativa dei con- 
temporanei, e a noi lettori dell'oggi, misurare le deviazioni verso un uso marcato, sia nel "passaggio da forme usuali a idiomatismi e a popolarismi tosco-fiorentini» sia nella «introduzione, in luogo delle forme e delle voci dell'uso, di forme e voci genericamente dotte e inconsuete». Non si tratta di un processo di correzione contraddittorio, ma chiasmatico: gli aulicismi e gli idiomatismi vengono sacrificati in quanto e dove fanno da attrito alla regolarità e all'espandersi di un uso hic et nunc e, verrebbe da aggiungere, sociale (si intenda partecipato almeno da tutti coloro che seguivano la espositione sopra la Commedia di Dante), uso che permette un successivo recupero di quelle categorie rimosse in altri contesti, e di misurarne, ora, da tutti e insieme lo scarto espressivo. La distanza tra fenomeni in apparenza analoghi e la convergenza tra fenomeni opposti (sollicito > sollecito // lecito > licito) sigla la diversa connotazione contestuale che la scrittura di P e di B consegnano alle medesime categorie storico-stilistiche, con richiami di estensione culturale e/o mimetica.

$\mathrm{Se}$, come è vero, la direzione negativa o positiva delle forme e delle voci dotte o idiomatiche di $\mathrm{P}$ non si sovrappone neppure in un caso a quella di B, è doveroso esemplificare, per non apparire il presuntuoso Calandrino che ha scoperto la pietra di paragone dell'espressività, le trame, i diagrammi, lo slalom (è permesso?) sui pendìi scritturali, per confermare quando la medesima linea segna la fuga o l'arrivo, ordina l'abbandono o promuove un invito.

Qualche esempio risponde a una domanda: sul perché l'autore, gli autori conducono alla soglia dell'incanto variantistico, mettono in mano una planimetria e si congedano quasi con una sfida maliziosa e seducente: ai lettori la scelta e il piacere degli itinerari.

Si scelga, in attesa dell'ora conviviale, la coppia manicare $>$ mangiare. In due casi B corregge, e attua quindi «un trapasso da una forma popolare e più rara a una forma corrente $\mathrm{e}$ 
usuale» (pp. 292-93). Ma, avverte l'esatto referto, in altri sei casi manicare rimane negli ingredienti della tavola espressiva boccacciana, sia pure minoritariamente rispetto a mangiare (come del resto accade nella Commedia, dove manicar è ben drammaticamente sulla bocca del conte Ugolino). La prima cancellazione di manicare si incontra in apertura, e incide con l'allotropo più peccaminoso nella confessione di Ser Ciappelletto: «alcuna volta gli era paruto migliore il manicare > mangiare che non pareva a lui che dovesse parere a chi digiuna per divozione, come digiunava egli». L'acquolina e l'attesa gastrica si contorcono nella masticatura sintattico-retorica della coniugazione poliptotica del parere: "gli era paruto [...] che non pareva [...] che dovesse parere», e manicare suonerebbe qui forse troppo saporito all'ascetismo del presunto digiunatore. Il frate confessore, un santo che non si apparenta con alcun «brodaiuolo manicator di torte» (III, 7 52), non respinge, quasi fosse tentazione, il gusto della forma più squisita $\mathrm{e}$ rara, e può mantenerla, poche righe dopo, senza schiocchi di lingua: "A ogni uomo avviene, quantunque santissimo sia, il parergli dopo lungo digiuno buono il manicare e dopo la fatica il bere». La sapidità di manicare - potenzialmente più raffinato e meno mandibolare di mangiare (stante la sopravvivenza di manicaretto) - è misurata e confermata da quella di bere: del resto, appena prima, il moribondo confessa di avere, in giorni di digiuno, bevuto acqua: «con quello diletto e con quello appetito l'acqua bevuta aveva, e spezialmente quando avesse alcuna fatica durata o adorando o andando in pellegrinaggio, che fanno i gran bevitori di vino». Sulle labbra di tanto attore, il venir meno della neutralità sinonimica qui e per lui appare dunque contestuale, legato alla esecuzione (visto che Arrigo Castellani - certo tra i destinatari ideali dei saggi di Maurizio Vitale - ci vieta performance).

Il secondo caso di sostituzione è nella novella di Bernabò Lomellin da Genova e della moglie Ginevra (II, 9): al pa- 
ragrafo 64 si leggeva in $\mathrm{P}$ e non più in B: «la fa uccidere e manicare > mangiare a' lupi». Al successivo paragrafo 68 si leggeva, come ancora si legge: «data a uccidere a un suo fante e a mangiare a' lupi».

La divergenza di P e l'equiparazione operata da B sembrano controllare una diversa sfumatura stilistica tra i due termini concorrenti, anche se nella novella di Pietro Boccamazza e dell'Agnolella sono ancora i lupi a preferire - nella degustazione ironica del parlante - il più appetitoso e antropomorfico «manicare»: "questo ronzino [...] noi cel mettemmo in casa acciò che i lupi nol manicassero» (v, 3 33; si veda al successivo paragrafo 46: «trovò pastori che mangiavano e davansi buon tempo»).

È la medesima ragione stilistica a determinare nell'insieme quell'accrescimento della patina fiorentina che B attesta, perché il Boccaccio, nel momento in cui una scripta letteraria municipale-regionale era accettata come lingua di una letteratura sovraregionale, ne potesse partecipare i tratti dell'uso vivo e sottolinearvi la vitalità del parlato. Esempio estremo, ancora nella sicura opzione del nostro studioso, data la ricorrenza plurima di "figliuolo» sulla bocca di monna Giovanna, l'irruzione della forma monottongata «figliulo» (p. 323):

$\mathrm{E}$ in così fatto pensiero impacciata, come che ella fosse certissima d'averlo se 'l domandasse, senza sapere che dover dire, non rispondeva al figliuolo ma si stava.

Ultimamente tanto la vinse l'amor del figliulo, che ella seco dispose, per contentarlo, che che esser ne dovesse, di non mandare ma d'andare ella medesima per esso e di recargliele, e risposegli: «Figliuol mio, confortati». (v, 9 I 5-I6)

Rilevato che nel passo citato, oltre l'idiomatismo del monottongo, $\mathrm{B}$ corregge anche andare in d'andare, più autoprescrittivo, si prenda atto del rimprovero che la voce narrante indirizza all'eccessivo amore della madre per il figlio, strap- 
pando al suo linguaggio segreto la variante idiomatica della possessione esclusiva: a brevissima distanza di due fra le tante occorrenze di "figliuolo», la strizzatina d'occhio vorrebbe virgolettare come riportato il sintagma «l'amor del figliulo»: la madre fiorentina non si tradisce, a farla tradire è il narratore.

Altre forme, «nella loro oltranza», sono classificate come «espressionistiche». Termine e concetto l'«espressionismo» che ha frequenze minime nelle analisi di Vitale, data la sua predilezione per «espressivismo»: ma si impone (a p. 549) ed è riferito a eccessi idiomatici, a cromatismi forti (si parla di "abusio vera e propria»), messi in rilievo dalla difficile armonizzazione dei registri. Alcuni dei casi esemplati, al di là di «messer lo ploposto» della Ciutazza (vIII, 4 32), contemplano una suggestiva complicanza non solo morfologica relativa al numero del più.

IV intr., 3 I

lasciamo stare l'avere conosciuti gli amorosi basciari e i piacevoli abbracciari e i congiugnimenti dilettevoli > lasciamo stare gli aver conosciuti $[\ldots]$

IV 3, 7

l'amor di tre giovani e d'altrettante donne, come di sopra dissi, per l'ira d'una di loro di felici essere divenuti infelicissimi intendo con la novella mostrarvi $>$ [...] di felice essere divenuti infelicissimi $[\ldots]$

v 6, 25

i due amanti [...] potessero esser veduti: e appresso fossero arsi sì come avevano meritato. > [...] in appresso fossero arsi sì come avea meritato.

VIII 2, 39

quanti moccoli raccoglieva in tutto l'anno d'offerta non valevano la metà di cinque lire [...] pensare in che modo riavere il [il tabarro] potesse senza costo $>[\ldots]$ ricoglieva $[\ldots]$ non valeva $[. .$.$] riaver lo$ potesse $[\ldots]$. 
Casi singolarmente discussi e interpretati da Branca anche nella sua edizione mondadoriana del Decameron (1976). Riuniti, come fa Vitale, in un insieme di confronto singolare / plurale, coinvolgono le valenze dell'azione amorosa, ma non nella reciprocità del baciarsi, dell'abbracciarsi, bensì nel grado zero del baciare, dell'abbracciare, dove i due (o più) attori sono significati dal morfema plurale attribuito e aggiunto a indeclinabili: un'azionalità di gruppo, zooantropomorfica, che, a ritroso, itera e moltiplica l'infinito passato sostantivato «l'avere conosciuti», rendendo la rigidità grammaticale dell'ausiliare, che la sincope («aver») salda al participio, fluida e isomorfa agli oggetti.

Folgorante la opposizione in $\mathrm{B}$ di una condizione di felicità di gruppo all'infelicità dei singoli, perché è il venire meno di un amore «felice» per l'ira di una persona che, spezzando l'unità 'singolare' del sentimento, contempla in pezzi la pluralità di tanti infelici. E se il prete di Varlungo muta prospettiva, focalizzando la somma e non gli addendi, «valeva» rispetto a "valevano», nel caso dei «due amanti» si scorge il rinvio a un principio giuridico ("come meritato"), alla colpa da punire, non alla responsabilità individuale di «ciascuno».

Il Decameron rimane una difficile quanto affascinante lettura scolastica, nella espressività stilistica perfezionata dalle revisioni d'autore, e ora accertata dalla filologia e interpretabile con i nuovi metodi critici. I volumi di Branca e Vitale possono essere utilizzati didatticamente, cioè a meglio capire e far capire lo «stilo» di un grande, tolto dalla realtà tragica e carnevalesca, cortese e mercantile. Si prelevino, ancora dalla novella di Federigo degli Alberighi, tre brevi passi:

7. rimase povero, senza altra cosa essergli rimasa che un suo picciol poderetto > rimase povero, senza altra cosa che un suo poderetto piccolo essergli rimasa.

29. se figliuoli avessi o avessi avuti, [...] mi pare esser certa che in parte m'avresti per iscusata $>[\ldots]$ mi parrebbe esser certa [...] 
34. pensando che voi qui alla mia povera casa venuta siate $>[\ldots]$ venuta siete.

Nella «esuberanza e vivacità espressiva propria della riscrittura» (p. 549) filtra quell'attenzione alla parola dei personaggi che porta a calibrare, con il timbro diatopico (certo, tutti pensano alla veneziana Lisetta) e sociale, il timbro emotivo. Naturalmente senza letture anacronistiche, perché il repertorio semantico, anche nella sua connotazione, è quello trecentesco: picciol e piccolo si stringono la mano nel loro status di variante foneticamente ma non sintatticamente adiafora, la loro diversa posizione nel sintagma e del sintagma nella frase, il contrapporsi topologico e semantico di «rimase» e «rimasa» dicono di una diversa convivenza di Federigo con la "cosa». E la delicatezza di monna Giovanna, che con il condizionale modalizza come eventuale e desiderabile la certezza di «essere iscusata»? E la compiaciuta rinuncia di Federigo alle regole astratte della consecutio, per collocare con l'indicativo l'evento nel suo tempo contestuale di accadimento?

Quando Vitale cita il Petrarca, il Petrarca suo, che parla di «varietas» - «morumque varietate stili varietas excusatur» - pone in rapporto lo stile ai costumi, cioè la diversità di luoghi, tempi, culture, sesso, carattere, situazioni, all'eterna difficoltà di rappresentare con la propria voce demiurgica le voci degli altri nella scrittura non loro.

Maurizio Vitale, memore dei suoi raffinati e anticipatori (e dunque ahimè lontani) studi sulla tradizione comico-realisti$\mathrm{ca}$, ha interpretato ritmi e armoniche, dissonanze comprese, dello spartito boccacciano, e lo ha sottoscritto, in una delle sue più plastiche sintesi, come passaggio dal monostilismo plurilinguistico di $\mathrm{P}$ al plurilinguismo monostilistico di $\mathrm{B}$.

Il medesimo tracciato che lo aveva portato a Manzoni, che da una parte cancellava il diritto alla conflittualità letteraria di lingua e dialetto realizzandone la convergenza estrema; 
dall'altra apriva, con le trasparenze del suo palinsesto plurilinguistico, la strada alla virtualità dialettale di Verga e all'espressionismo gaddiano con il suo (di Gadda) perenne senso di colpa verso quel maestro che in una sola lingua - nella linea del grande e meditato certaldese - aveva scritto le parole di tutti:

Al terzo, come al primo, la maggiore abbondanza e varietà venne dalla natura degli argomenti; fatti, detti, costumi d'ogni genere. [...] quante forme di concetti, quante attitudini di linguaggio, in tanti e sentimenti e discorsi e vicende, di principi, di cavalieri, di gentildonne e di donne d'ogni condizione, d'uomini di corte e d'uomini di villa, di boni e di tristi, di generosi e d'abiettti, d'astuti e di sciocchi, di scienziati, di scolari, di corsari, di banditi! (Appendice alla Relazione, IV, I I)

3. Solo dopo le storiche pagine su Petrarca e su Tasso, e le ricchezze di poesia tesaurizzate, con la guida, lo schema e si direbbe lo schermo del cantore di Laura e del cantore di Silvia, di Clorinda e di Erminia, Vitale poteva affrontare il percorso impervio e inamabile del poema di Gian Giorgio Trissino, stampato nel biennio maggio I 547 -ottobre I 548 , in tre tomi, il primo a Roma, il secondo e il terzo a Venezia, dal Tipografo da lui prediletto e tormentato, Tolomeo Ianiculo da Brescia, o meglio «da Bressa», la città che nel poema viene onorata dell'epiteto «fertilissima» (x 392; XII 3 Io). Ma le date di stampa non ingannino: anche il poema nasceva negli intensi anni Venti trissiniani, se Giovanni Rucellai, «il Castellano» (morto il 3 aprile I 525), nell'offrire all'amico Le Api, scrive:

Deh poni alquanto, per mi' amor, da parte il regal ostro e i tragici coturni della tua lacrimabil Sofonisba, e quel gran Belisario che frenando i Gotti, pose Esperia in libertade, o chiarissimo onor dell'età nostra. (vv. 64-69) 
A séguito dei due monumentali saggi dedicati, nell'arco di un decennio, come detto, ai Rerum Vulgarium Fragmenta (1996) e alla Gerusalemme liberata (2007), il piacere intellettuale procurato dal computo dei fenomeni grammaticali e dalla scomposizione delle tessere retoriche, per attingere ai nuclei della ratio della poesia petrarchesca e tassiana, sembra avere imposto il complemento di un esercizio penitenziale: ecco L'Omerida italico: Gian Giorgio Trissino. Appunti sulla lingua dell'«Italia liberata da' Gotthi» (2010), ancora una volta un confronto con un'opera difficile, rimossa dalla vulgata critica, «antipoetica», per richiamare il lapidario giudizio di Carlo Dionisotti (I980).

La certezza che Vitale possa distillarne, con suggerimenti discreti, un quod sufficit di fascino espressivo tra i filari dei ventisette canti popolati da Goti e Bizantini aridi di poesia, invita gli studiosi a ricostruire globalmente la figura di questo protagonista, appartato e, come appena detto, emarginato, ma originalissimo del nostro pieno Rinascimento, e recuperare il significato della sua presenza: di scrittore insieme parco e prolifico, che si è provato nella commedia, nella tragedia, nella lirica, nell'epica, nella teoria linguistica e retorica, senza ripetersi, un testo per ogni genere. Trissino concentra in due anni una intensa attività poetica e trattatistica, tipograficamente unica (come la mostra testimonia): nel I 524, a Roma, con i tipografi Ludovico degli Arrighi Vicentino e Lautizio Perugino (Canzone a Clemente VII; Epistola de le lettere nuovamente aggiunte ne la lingua italiana; Sofonisba; Ritratti; Orattione ad Andrea Gritti); nel I 529, a Vicenza, con il citato Ianiculo (Epistola de le lettere nuovamente aggiunte ne la lingua italiana; De la volgare eloquenza; Il Castellano; Poetica; Grammatichetta; Rime; Sofonisba), per giungere, in un silenzio non inoperoso di quasi venti anni, alla chiusura e alla stampa del poema, cui seguiranno la commedia Similini ( I 548) e, postume, le parti quinta e sesta della Poetica (I 562).

Dalla Questione della lingua (ci si riferisce sempre alla edi- 
zione I978, nella ristampa I984) era ribadito che Trissino rimane nella storia, più che della poesia, della lingua italiana, per la sua sfida al primato della grammatica della Firenze a lui contemporanea, di Petrarca nella tradizione lirica, di Virgilio, come supremo modello, per la naturale osmosi di lingua e linguaggio, allo "stilo" dei nostri classici. Sfide dunque all'amico e quasi conterraneo Pietro Bembo, al ferrarese Ludovico Ariosto, al mantovano Baldassar Castiglione, tutte perdute. Gli rimane il merito della traduzione del De vulgari eloquentia di Dante, della provocatoria proposta (già avanzata, in un manoscritto riscoperto solo alcuni decenni fa, da Leon Battista Alberti) di un sistema grafico fonologico che distinguesse - nel sostanziale rispetto della fonetica fiorentina - anzitutto le vocali aperte dalle chiuse, le sibilanti e affricate sorde dalle sonore, $c$ palatale da $k$ velare (kjesa), per sostituire al trigramma $g l i$ per la laterale palatale il digramma $l j$ : sistema grafico subito obliterato dagli editori, ma tenacemente restaurato da Vitale in tutte le citazioni che dànno corpo a queste sue pagine saggistiche (con puntuale rilievo della evoluzione e delle incertezze 'ondivaghe' del sistema stesso).

Il poema, come del resto gli altri scritti dell'umanista Vicentino - sono informazioni del primo breve capitolo, Preliminari. Il Testo - dopo la riedizione Verona, Vallarsi, I729 (da cui si deve citare, pur non rispettando la veste grafica e anche fonetica della princeps), nel corpus dell'opera omnia, vedrà, in Italia, una censurata offerta per esigui lettori nel I 835, nella già sua Venezia, a un secolo e a decenni dalle due edizioni 'straniere', di Parigi (I729) e di Orléans (I787). Una quinta e importante edizione reca l'indicazione tipografica di Londra, I779, e anche le schede dell'ICcu la collocano in Gran Bretagna: ma l'avvertimento «Si vende in Livorno presso Giovanni Tommaso Masi e Compagni» certifica, qui come in altri ben noti casi, che i tre volumi sono stampati in Italia, e fanno parte della serie dei classici che, tra il 1778 e il I 784 , vedeva 
uscire nella città labronica - dove nel 1764 era stato stampato, da Marco Coltellini, un anonimo trattato, Dei delitti e delle pene - le opere di Dante, Guarini, Petrarca, Pulci, Tasso, Tassoni, Trissino appunto, Alamanni, Forteguerri, Berni, Chiabrera, Filicaia, Tansillo, Algarotti, un canone in cui oggi si riconoscerebbero come classici forse solo gli autori della Commedia, dei Rerum Vulgaria Fragmenta, e delle due Gerusalemme, la liberata e la conquistata: autori e titoli su cui altrove, anche nell'età dei lumi, non era da escludere qualche riserva censoria.

A un problema di censura, che coinvolgeva direttamente Trissino richiama preliminarmente Vitale (p. 5, dove rinvia a un saggio successivo), rilevando come della princeps «esistono [...] esemplari con le invettive antipretesche ed esemplari senza quelle invettive»: in una tiratura mancano trenta versi (570-599) del libro Xvi con la violentissima polemica verso la curia romana e i pastori della Chiesa intenti «ad aggrandire i suoi bastardi, e darli / Ducadi, e Signorie, Terre, e Paesi, / e conciedere ancor senza vergogna, / Prelatura, e Capelli a i lor cinedi», dunque verso gli amici e protettori Leone $\mathrm{x}$, Clemente vir e soprattutto Paolo III, che pochi anni prima, il 23 settembre I 545, aveva imposto il figlio Pier Luigi Farnese come duca di Parma e Piacenza. La distaccata dignità politica di Trissino sfocia in una invettiva 'luterana', che si coniuga coerentemente alla sua altera solitudine umana (si veda l'appendice testamentaria, pp. 21 5-28), e di innovatore eretico nelle contese delle humanae litterae del pieno Rinascimento.

L'appellativo antonomastico per l'aspirante poeta vicentino di omerida italico orienta la verifica sullo stile e sulla grammatica della sua tentata riforma all'interno del genere cavalleresco corretto in epico, ma non va assunto nella più facile accezione di 'epigono o imitatore di Omero', né lo colloca tra $\mathrm{i}$ "poeti moderni, che si ispirano al mondo omerico e, in generale, ellenico, o rinnovano antiche, gloriose tradizioni 
del proprio popolo», come spiega GDLI, con un passo di Eugenio Montale, che ne onora Mistral. Nell'ampio orizzonte rinascimentale contemplato, essere omerida significa ricreare competitivamente «il divino Omero», e alzare il proprio poema in endecasillabi sciolti al di sopra della serie - si oserebbe serial - cavalleresca in ottave.

Quindi, a contenuto, un argomento bellico quasi ignorato dalla storiografia rinascimentale, ma documentato da fonti inoppugnabili, ad altri inaccessibili, La Guerra gotica del greco Procopio, l'Epitome rei militaris del latino Vegezio, la Téchne taktiké di Arriano; una vicenda bellica di liberazione, non di conquista: i campi in parte sinonimici in parte antinomici della 'guerra giusta' se non 'santa', un binomio scomposto dal tormentato scrupolo semantico di Tasso: per lui non sarà necessaria la restrizione di un complemento di rimozione (non di azione) quale era "da' Gotthi»: se per Gerusalemme si sarebbe rivelato irrituale e impoetico precisare il nome dello straniero da scacciare, l'assoluto infedele, per l'Italia pontificia e spagnola occorreva risalire a un invasore antico, secondario, al momento in cui, pur disgregatosi l'impero romano d'Occidente, la penisola poteva essere - per l'ultima volta - legittimamente indicata come Italia, e non ancora come Longobardia e Romania, o dai nomi in sequenza geografica e cronologica dei suoi municipi e delle sue signorie. L'unitario nome geografico consuonava all'auspicio, intercettabile nella dedica a Carlo v "Massimo», nuovo Giustiniano, restauratore di una confederata unità nazionale, certo non il principe voluto da Machiavelli. Trissino, che si pone come continuatore degli auspici di Dante e Petrarca, celebra degli eroi «ch'andaro a liberar l'Italia oppressa» (II I 38 e passim) - offrendo un endecasillabo da iscriversi tra gli slogan risorgimentali, se il poema avesse trovato un lettore nell'età di Manzoni (nella sua biblioteca solo Il Castellano), di Cattaneo e di Verdi.

Tra le riflessioni dichiarate e suggerite dal secondo capito- 
lo, Orientamenti culturali e intenti poetici (pp. 9-18), coinvolge e persuade la illustrazione delle derivazioni dei personaggi trissiniani da quelli omerici: Agamennone, Aiace, Ulisse, Patroclo, hanno nell' Italia liberata una loro moderna controfigura, e perfino l'ha Tersite: «Anticalo fremeva, / ch'era di sangue assai famoso, e chiaro; / ma di parole inordinate, e molte, / e poco riputate da la gente. / Questi era il più brutt'uom, che fosse in Roma, / guercio, e sottil di gambe, e le sue spalle / gobbe, pareano quasi arco del petto» (pp. I6-I7 [XIV 76I67]).

Centrale nel saggio l'ampio capitolo terzo (pp. 19-208), dal titolo La forma espressiva, perché è qui che si scandisce il passo trissiniano sull'orma omerica, nella garante cornice della precettistica poetica ed ermeneutica di Aristotele. L'enérgheia dello storico della lingua - ben nota, ma qui in competizione con quella del suo poeta, a mostrare il viso dell'arme sia al precedente non predecessore Petrarca, sia al dissidente 'continuatore' Tasso - procede con la riconosciuta millimetrica dissezione del corpo fonetico e morfologico della parola, con sensibilità culturale ed estetica degli spessori del significato, facendo reagire i dati testuali nella collaudata complementare bipolarità della «sentenza» e della «elocuzione». Il lettore del saggio tassiano sa che i due termini sono aristotelici, e separano, diremmo oggi, lo stile dalla grammatica, la testualità dalla sintassi, la dicitura (écriture) dalla lingua, per rincorrere, con dicitura, il tecnicismo iponimo e quasi sinonimico a sentenza, con cui Vitale apre la sintesi conclusiva del capitolo IV e ultimo (Il sistema linguistico trissiniano nel disegno omerico, pp. 209-I 4): «La dicitura del Trissino nell'Italia liberata appare [...] di originale e singolare confezione. Essa si avvale di una sentenza pienamente conforme alle sue concezioni teoriche e alle sue dichiarazioni programmatiche e di una lingua del tutto consona alle sue posizioni linguistiche concettuali e prescrittive». 
L'obiettivo sperimentale perseguito da Trissino, fedele al predicato realismo del suo Omero, si realizza in una prosodia endecasillabica, dunque paraesametrica, su un registro tonalmente prosaico, che dichiara e non sempre accende l'epicità poetica. E, come sempre, Vitale non proclama, parametra, esemplifica, classifica: sollecita il lettore all'intelligenza della grammatica, dell'espressione e delle densità intratestuali, anche dove accerta che l'ispirazione non stacca i piedi da terra, elevandosi a un confronto solo 'comparativo' con il modello, raramente toccando il sovrasenso metaforico e simbolico.

Rinviando al saggio per gli spogli delle strutture retoriche come l'iperbole o l'accumulazione, dunque prevalentemente semantiche, o come l'iperbato, prevalentemente sintattico, spesso compresenti e cooperanti - «e si colpir con sì terribil colpi, / che parean proprio fulguri, o bombarde, / ch'urtino i sassi, e gli albori, e le torri» (xxIII 574-76), "perché la passion l'ingegno offusca, / e 'l giudizio impedisce, e la prudenza» (XXIV I I 2-I3) - ci si sofferma sulla comparazione, con esiti spesso non indegni di Omero (pp. 44-57).

I termini di paragone riportano molto spesso a un'area indigena, alla campagna veneta (e si pensa agli scorci naturalistici dei suoi grandi pittori), al cortile più che alla corte, $\mathrm{e}$ dunque a un realismo che si attesta in dialettalismi nobilitati. Cosi la vegetazione comprende, con le robuste quercie, i faggi, gli olmi, un'alta pioppa (due occorrenze). Gli animali laureati dall'epica, i leoni e gli orsi, i veltri, le colombe, i cigni convivono con le meno nobili anitre (avrebbe scritto Giorgio Orelli che questi anseriformi esigono la $i$ della trafittura e della illuminazione), con gli anadrotti, e le galline, un pollame contadino insomma, i cani, ma "con la coda ristretta fra le gambe», i conigli, i ranocchi e perfino le mosche, e con i sassi i cogoletti: 


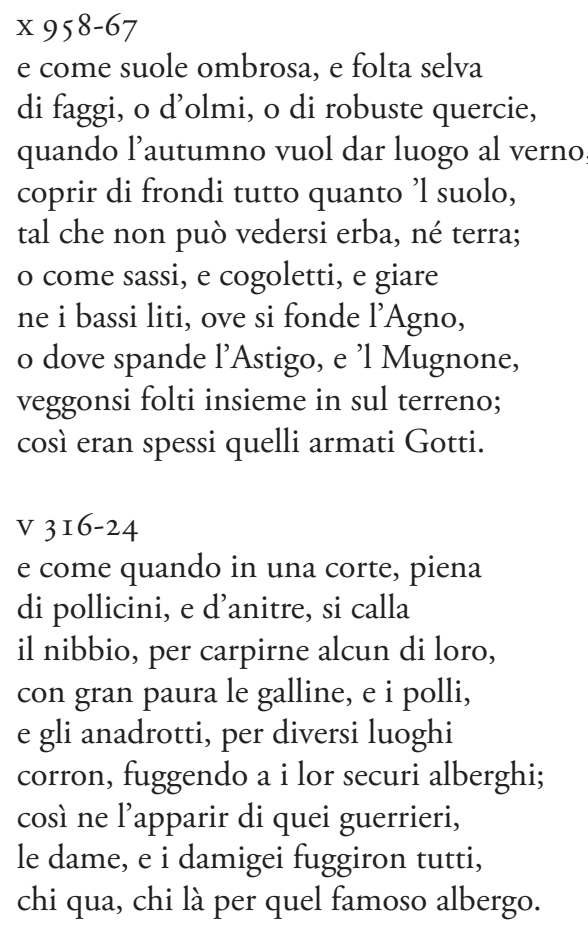

Dalle risorgive di Basiglio il lettore viene invitato a seguire - e qui si calcano con maggiore aderenza le orme di Omero - un fiero cavallo, quasi staccato dalla terra (lo stacco è dato dall'inciso non rilevato dalle virgole nell'ultimo verso), al galoppo nella libera natura delle sue praterie:

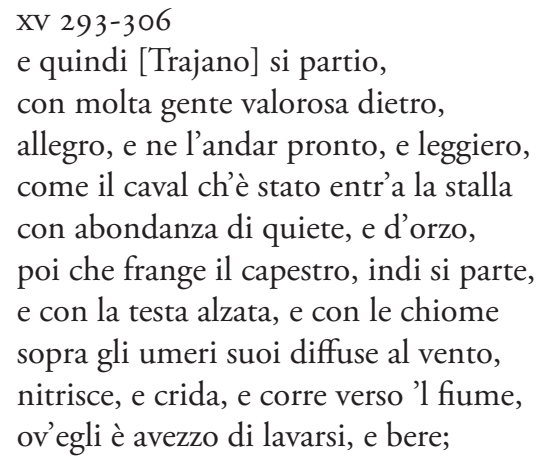


e vago, e lieto de la sua belleza,

sì leggiermente le genocchia innalza, per entro 'l piano, e per gli usati paschi, ch'appena tocca con le piante il suolo.

Gli accademici delle scienze sarebbero certo più coinvolti dal raggio luminoso che si rifrange nell'acqua di un vaso posto in alto nella penombra di un palco a soffitto (III 238-45: «se in un capace vaso, / che sia pien d'acqua tremolante, e pura, / entra il raggio del sol, che si rifletta / nel palco, e sotto le aggravate travi»), come il pensiero della «vaga, e giovinetta amante» Sofia; o dal bagliore del fuoco sotto la cenere, dalla violenza delle onde del mare, dall'irruenza di un torrente sulle campagne devastate (vII 69I-98), emersa la pietas per i contadini: «onde l'agricoltor si batte l'anca, / vedendo gir le sue fatiche a terra».

Le preziose collaboratrici familiari, a loro volta, potrebbero fare tesoro - con legittimi dubbi sulla disarmonia delle due situazioni a paragone - del prezioso interno casalingo, al riparo dal gelo invernale, forse ripreso da tela di pittore della realtà lombarda più che venetica:

Xv 590-600:

Come la fanticella, quando bolle

la pentola sul fuoco, e spande fuori

l'onda gonfiata, e la bollente schiuma,

corre a la secchia, e prende gelid'acqua

con la caccia di rame, e porta quella

per l'aspro fummo, e ponla entr'al pajuolo,

onde s'acqueta il suo bollir feroce;

così que' dui Baron, quando portaro

per l'empia nube di saette gotte,

la grave statua, e la gettaro a basso,

s'acquetò il gran furor di quella gente.

In questi significativi lacerti testuali, misurati i trabocchi dei termini di paragone, è facile - guidati dalla chirurgica incisi- 
vità dello spoglio - distinguere i materiali della costruzione espressiva, e insieme la grammatica, come si ricompongono nella seconda parte di questo terzo capitolo, dedicata alla «elocuzione». Si illustra come Trissino attinga, con misura di cultismo se non paritetica omologa, dal greco e dal latino, e dal contesto indigeno in cui vive e opera, resistente alla tentazione edonistica (per ricordare la fondamentale categoria definita da Cesare Segre), forse non immune dal preziosismo antiquario del restauratore (rappezzatore?) che, nel mosaico di vocaboli culti rustici tecnicamente precisi, per l'eccezionale come per il quotidiano, spartisce note distoniche: con il sergente e il caporale e il colonnello, tra i graduati dell'esercito, si incontrano il decure e il tergiduttore; e nella truppa, con i principali (i latini principes) e i triari, gli strasordenari, con le centurie e le turme le bande. Era il calibro della langue grammaticalmente 'comune', non solo cortigiana - come esigeva il suo De vulgari eloquentia - per l'estensione della dicitura, mista la grammatica, federativo lo stile.

In questa sua fatica - e credo che il termine sia condivisibile, se precisato da almeno due aggettivi, appassionata e felice - Vitale, pur in una ritenuta prudenza sulla poesia epica trissiniana, raccoglie e offre elementi per correggere la severità del giudizio vulgato. Certo la «forma espressiva» è umanisticamente artigianale, e quasi disturbano i rari abbandoni alle seduzioni per esempio allitterative, essendo qui vietato parlare delle autonomie del significante, come in due segmenti provocatorî:

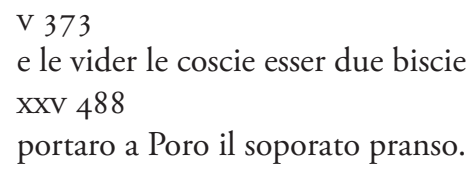

$\mathrm{Ci}$ viene insomma consegnato un Trissino documentarista e regista che, una volta inquadrato il campo, lascia fare alla 
macchina da presa. Lui, che attingeva da Vitruvio il lessico architettonico (pp. I96-200), e che ispirava a Palladio l'accordo della proporzione classica al contesto ambientale, con l'armonica dissonanza della sua scansione prosastica, ricorda, per rimanere nel territorio della promozione artistica, un pittore che in un tratto si ferma alla monocromia per instillare il desiderium del colore, in un altro eccede e satura il disegno di violenze cromatiche; uno scultore che per il suo monumento «aere perennius» opta per materiali ferrosi, calcolate schegge, frastagliate le superfici, appuntiti gli spigoli, prefigurata sulle volute sconnessioni la coesione seduttiva della ruggine.

4. Insisterebbe una piccola ombra, in questa festiva riflessione critica sull'opera del nostro Maestro, se non si accennasse alla sua empatica lettura e cura filologica dell'opera di Riccardo Bacchelli, che ci sollecita da via Borgonuovo, dove era la casa della sua adozione milanese, e da questo nostro Istituto, nella cui tradizione lo scrittore bolognese si è sentito onorato di entrare e farne parte, come illustrato dai documenti e dai testi che siglano l'ultimo capitolo della mostra qui presentata.

La tenace e solitaria fedeltà a Bacchelli è il punto prospettico, la postazione moralmente polemica, da cui Vitale ha guardato e guarda alla contemporaneità del Novecento, e ne interpreta i valori ideologici e gli esiti espressivi. Si comprende come la sua cognizione, profonda e aggiornata, della letteratura italiana e delle letterature straniere, nota a chi ha la fortuna di un dialogo diretto, derivi un canone autoriale fondato sulle intertestualità stilistiche del nuovo con la tradizione, la intertestualità positiva e, più virtuosistica, la negativa (I, 9-IO [si cita dall'edizione Mondadori I957]).

La gran ruota, l'ulà, come la chiamano, gira coll'andar del fiume reale, e la sua lenta cadenza diventa tutta alacre nella macina soprana, alata (e non chiamano ala, i mugnai, il giro esterno di essa?). 
Da questo passo, del Prologo al fluviale romanzo, o meglio al "poema molinaresco" - sottotitolato Quasi una fantasia (Delio Tessa non contrappuntava, con la cautela di un milanese quasi italiano, Sonada, quasi ona fantasia, il titolo del suo poema sulla ritirata di Caporetto, sofferta e narrata dallo stesso Bacchelli?) - Vitale, nella monografia Sul fume reale. La lingua del «Mulino del Po» (I999) - dedicata "Alla memoria di Ada Fochesati musa e consorte di Riccardo Bacchelli» -, ha guardato, unendo le rive semantiche della storia letteratura e della cultura artigiana e dialettale, all'intrecciarsi, non al mescolarsi, nella scrittura di «tradizione e modernità».

Il tecnicismo ulà, che preannuncia altri stilemi seduttivi, non è rivelato dal corsivo, perché qui tutte le parole, quelle prelevate sulla bocca di Lazzaro Scacerni o dalla pagina di Manzoni, o ancora da una più segreta tradizione prosastica, si rivestono di una medesima dignità, quella della 'normalită' letteraria. La successione prosodica, appena mascherata dalle cadenze sintattiche, del casuale endecasillabo sdrucciolo di apertura, se non avesse al séguito - non si dice a ruota - l'endecasillabo piano, più regolare, se non fosse quella sincope motoria di andar e l'accento di settima su fiume; replicato in successione con rima e assonanza interna - «e la sua lenta cadenza diventa» - che si protende nella clausola dodecasillaba, dove alacre (parossitono o proparossitono?) tende una mano, etimologica o paraetimologica, ad alata, in un impossibile ma desiderato - la parentesi e la virgola lo dicono - abbrivo di volo, quasi la ruota fosse mossa dal vento.

Il saggio, schivo, didascalico nei titoli, sembra resistere alle tentazioni di madonna allusività: manda in avanscoperta, sulla orchestrata linearità della pagina, la donna dello schermo, l'eternamente nubile grammatica, magari quella agghindata dal suo Cecco Angiolieri: per le frequentazioni mercantili e furfantesche di alcuni utenti del Delta padano. Con lei, come sempre, si accede ai tre capitoli che separano le componenti 
della letterarietà, della popolarità, del realismo storico e ambientale, rispettivamente per cento, cinquanta e quaranta pagine, come a dire - anche con l'imparzialità dei numeri - che la letterarietà fa aggio, come la farina sulla crusca, quest'ultima in partibus fidelium - tacito il consenso del «nitido» Accademico - apprezzata, se non più, almeno quanto «il più bel fior».

Eletto, sostenuto, marcato, fluente, dovizioso sono i termini impliciti ed espliciti di una valutazione critica sempre puntuale e tesa, che impone al lettore il registro alto, lo introduce alla lettura provveduto delle notizie necessarie a percepire una aulicità, un ornato, dei colori che i protagonisti della storia vorrebbero fossero loro, riconoscendovi gli abiti della assunzione epica con cui lo scrittore riveste la loro rustica dignità. Si prelevi, come esempio, a fronte delle indicazioni enucleate, della «opulenza linguistica», della "notabile eloquente esuberanza lessicale», dei «cumuli verbali», e della loro distensione orizzontale negli iperbati, nelle bilanciate distanze degli elementi coreferenti, nello scarto degli incisi, dalla levitazione traslata dei semantemi materiali, un passo espressivamente quasi neutro.

E da una boscaglia in golena sorgeva con agile impeto un pioppo a meraviglia alto e snello, affusolato, che ergeva in cielo la vetta e il tenero virgulto, in cui s'ingentilisce alla cima la gran forza saliente, lo stollo fogliuto ed ardito: nel cielo la ergeva, docile ai respiri dell'aria più lene, dal gran piano, dove la natura sembra che renda in profondo quanto leva di largo, e che dal precluso orizzonte richiami e drizzi ed intenda allo zenit, facendo più alto il cielo e più vicino insieme; sicché talvolta l'uomo sente voglia di sdraiarsi col volto e cogli occhi al cielo, e sprofondandosi si perde e riposa con l'animo nella luminosa cecità del vertice azzurro. (III, I90)

L'elemento più rilevabile, con fogliuto, è saliente, nella serie suffissale che accoglie vocaboli «meno consueti», come fastidiente, negghiente, ridolente, ecc. (p. 43). Si avvertono le ripetizioni, a distanza che sembra d'inerzia, della parola cielo: ma 
eccola incrociata dalla retorica del chiasmo: «ergeva in cielo la vetta ... nel cielo la ergeva»; l'impennata in climax della "natura», che sembra cercare compagni e poi sale da sola verso un altro cielo, a superare la dittologia ossimorica verticale di alto e di basso, nel 'sinonimico' «vicino" che presuppone l'uomo e un quarto cielo, il suo, dove non è adinato né ossimoro lo sprofondarsi nel vertice, atto solo spirituale. La trasparente filigrana leopardiana affiora qua e là, il picco più vistoso è da "quel precluso orizzonte» verso «l'ultimo orizzonte», fino al naufragio nell'alto, al perdersi nella superiore "luminosa» infinità. Lo sfumato riferimento intertestuale, per riecheggiare in verbo l'aggettivo di Bacchelli, 'lenisce' l'aggressione descrittiva, volta ad accostare, non rivelare, l'allusione culta alla nebulosa percezione memoriale dei popolani.

Aprendo una parentesi 'grammaticale', si annota che la $s$ - di sprofondandosi, secondo un'attempata stilistica, è «intensificativa", ma sarà sommersa, in un bilancio finale, dalle occorrenze di fratelli e sorelle «negativi» (a volte "fattitivi»): se non il nostalgico smemorare, certo i neologici e solo bacchelliani srepubblicanandosi (III, I92), detto del "partito radicale democratico, che veniva srepubblicanandosi d'anno in anno collo stare al potere»: gli invitanti sradicalizzarsi, sdemocratizzarsi, e, per revisione inerte, desinistrizzarsi - da interpretare, con sollievo, "uscire dai sinistri" -, a scasarsi (III, 200: «i più erano stati scasati dal fiume»), conversione a calco del dialettale stamplinar "sgombrar casa"”, recuperato a glossa dal narratore: come sottolineato dal suo critico (p. I78). Più scontato, e appena da avvertire, l'incontro di in-negativo con i concorrenti prevalenti e forti illativi (inciprignita, insolfanata), che quasi si eclissa in pagine coperte da un grigiore cronachistico, sommesse e sommerse, come quelle dedicate all'amore sfortunato di Orbino per Berta Scacerni: lei «più inattingibile che mai», lui innamorato e ingelosito (III, 435).

Nei rilievi sostanzialmente sintattici e lessicali che 
costituiscono - insieme al "contrappunto» paremiologico e sentenzioso - la trama della "popolarità», Vitale nota che «il dialetto [...] resta [...] sostanzialmente estraneo al discorso dialogato del romanzo» (p. I 35). La "popolarità» è cadenza, pacatezza, stilema familiare che Lazzaro si impone, fin dalle prime battute, e sembra trasmettere alla discendenza, buona e cattiva, naturale e spirituale: «Le lettere dell'alfabeto, il mio uomo, sono la bellezza di ventiquattro, due dozzine» precisa "con superiorità degnevole il bottegaio" (I, 80: per i suffissi in -evole, vd. p. 39) con la formula amicale, in realtà padronale, dell'articolo necessario al possessivo: «il mio uomo». Per ristabilire le distanze e, soprattutto, a fissare le gerarchie, Lazzaro ricorre a modo analogo, dove è il deittico a marcare il percorso e i moti divergenti dell'allocuzione:

E a voi, quell'uomo, a che serve sapere i fatti miei? Avete mai sentito di quello che sotto la forca domandò "Il nodo scorre?". [...] Sono puntiglioso come quello del nodo, che vi auguro presto. (I, 8I)

Il deittico individua - giocando sulla approssimazione che il popolano finge di non sapere rimediare - e proietta sullo sfondo esemplificativo due uomini, i protagonisti del dialogo, "quell'uomo», che è l'altro, e "quello del nodo», controfigura dell'io parlante, che, tramite il relativo e la pausa attante della virgola, passa «il nodo» all'interlocutore. Moduli semplici, naturali alla pacatezza ferrarese - ironica e connotata da quell'accento silenzioso cui Raf Vallone seppe cosi bene dare voce - sono la tematizzazione, con ripresa pronominale, magari con tema sospeso, l'e ipotattico, i dimostrativi rafforzati dall'avverbio, e altre riprese del parlato. Eccezione è l'irregolarità sintattica vera e propria, ben segnalata nel saggio, messa in bocca a Giovanni, di fronte al sergente che indaga sull'incendio del mulino San Michele: 
Vuol dire che avessimo dato fuoco a un mulino, anche se i contatori non fossero stati in regola, per risparmiare qualche centinaio di lire di multa. (III, 240)

Congiuntivo del dialetto e condizionale della lingua erano facile sesto grado cui appendere la provocazione, di li a poco replicata con un inconsueto gioco parafonico: «recidivi» dell'ufficiale provoca una domanda: «Redici... revidici... recidivi, lei perdoni l'ignoranza, che cosa vuol dire?» (III, 24I). Viene in mente un capolavoro dialettale del seicento romagnolo, la $\mathrm{Ba}$ tistonata, dove il popolano chiama - fingendo di sbagliare e sbagliandosi - un eselent, un 'eccellente', inselent, 'insolente'.

Del viaggio di Princivalle, come Manzoni per quello di padre Cristoforo, il narratore Bacchelli approfitta per alcune divagazioni storiche, non sulla vita di Ludovico, ma sulla storia risorgimentale d'Italia: «Intanto Princivalle Scacerni era arrivato alla Guarda» (III, 300). E se un lettore si azzarda a rilevare che $" \mathrm{Ci}$ sarebbe arrivato dieci volte [...], con tutte queste digressioni», ecco un'utile occasione per rivelare il segreto della scrittura:

Non sono digressioni, È cercar le cose per intiero [...]. E se non avesse per avventura, del ricercato e del sottile, aggiungerei che vuol essere, nel raccontare, lo stile del contrappunto.

È la presentazione di quei meccanismi interdiscorsivi e più sottilmente intertestuali che Vitale individua, anche lui, con la discreta sapienza del contrappuntista, sintetizzando, da storico della lingua qual è, la calibratura tra la letterarietà e la "costruita usualità incolta» del pigmento dialettale: «Il multiloquio, anche fra individui di ceti diversi e di differente cultura, ha la sua base nel lessico dimesso e a volte corposo della parlata corrente, istituita come lingua dello scambio abituale e comune» (p. 1555: si scorra al mistilinguismo anticipato, nel cultismo e nella naturalità 'maremmana', del lessico della 
prosa epistolare di Carducci [in Divagazioni linguistiche dal Trecento al Novecento, Firenze, Cesati, 2006, pp. I39-84]). Ma insieme base di uno stacco verso un registro più alto, realistico anche dove in altro scrittore parrebbe metaforico.

Così, «i frequenti verbi frasali» del sotteso parlato dialettale si connotano talvolta come «usanze ineleganti», tipo parar sù, o scapolarne fuori, quelle forme insomma che il Ponza rimproverava a Grossi per rimproverare a Manzoni: ma la misura di sorger fuori applica sul quasi polirema, sul 'venir al di sopra dell'acqua, fuori dalla sepoltura dell'acqua', la ricchezza poetica del 'risorgere': «Sorse fuori tutto allegro, scrollando, e spremendo l'acqua colle mani dai panni» (III, 735): è l'ultimo Scacerni, che nell'epilogo muore sul Piave, quasi a scontare la salvezza di Lazzaro sulle rive del Vop.

Questo sorgere fuori dialoga, intratestuale, con il grado zero e con quello sintagmatico del verbo, nella verghiana conclusione del romanzo, al ripescaggio del cadavere dell'Orbino ucciso da Princivalle Scacerni. (III, 666-70)

«Stanotte o domani, domani notte alla più lunga, lui sorgerà [...] tu mi puoi dire di sicuro dove lo porterà il fiume a sorgere» «sorge in questi due posti, se la piena non lo porta sino al mare» «Ma tu non hai fatto ancora quello che gli hai giurato. Ma i morti vogliono uno dei loro. Uno che gli abbia voluto bene, quando sorgono»

"Vuole lei, per quando sorgerà, lei, che si voglion tanto bene» «E nel fiume, lui; Scansafrasca sa dove sorge: ha da riceverlo Berta, che gli vuol bene; se no, s'offende»

"Andate con Scansafrasca, che sa dove sorgerà»

«Ma lui può sorgere fra poco».

L'ecolalia, illustrata con ricca e pertinente esemplificazione nel fraseggio individuale («So quel che dico: anche quando dormo mi spia! So quel che dico»), diviene ecolalia dialogica, a distanza di rimbalzi, non in ripetizione sintattica delle parole, ma in cadenze di scomparsa e di emersione emotiva, di 
rimorso e speranza. La grammatica della retorica svela il suo segreto stilistico. (III, 675-77)

Sorgeva l'ucciso.

"Se sto qui, lo offendo: non ha da incontrar la mia faccia ora che sorge».

II sole ardente già l'asciugava; l'acqua l'aveva lavato, quasi piamente, e gli aveva abbassate le palpebre, ravviati i giovani capelli, che celavano la ferita della nuca, Del resto, il volto era intatto, e della morte esso e la struttura della sua più nobil parte riceveva forza, ch'era terribile e serena, eterna sotto il sole perituro. Bello era più che da vivo; la morte ha tocco così leggiero e pietoso da ottener perdono da chi niente altro più invidia.

La filigrana è pluritestuale: la dieresi nascosta su «pïamente» avverte di Dante, del suo Manzoni, di Pascoli, del suo Carducci, nella piazza di San Petronio (con «il sol morituro»); qui, come altrove, «il multilinguismo e il multistilismo dell'opera appaiono risolti e assorbiti, con limpida coesione, in una cifra personale di inconfondibile efficacia espressiva, nella quale singolare forbita signorilità, accurata proprietà terminologica e fluente opulenza costruiscono» quella confectio linguistica felicemente sintetizzata in "policroma struttura ognora artisticamente omogenea» (pp. 225-26).

La inevitabile prospettiva di fine secolo non deve impedire - questo l'appello accorato di Vitale nella sua sicura attraversata storico-linguistuca - di collocare questo forte romanzo, che celebra la nobiltà della «rustica prole», nella dignità di un linguaggio per il quale la critica, pur prendendo atto delle molte citazioni del Grande Dizionario della Lingua italiana, non ha saputo, nell'àmbito degli -ismi novecenteschi, applicare la definizione rivelatrice. Un popolo coraggioso, misurato e violento, sentimentale e concreto, nel crepuscolo della sua storia e al confluire della nostra tradizione letteraria in una discorsività culturale ecumenica, veniva 'cantato', nella concertazione contrappuntistica delle voci educate dell'acca- 
demia a quelle fresche e sfrontate della strada, nel comporsi di una sinfonia intensa variata satura, che mostrasse quanto ancora poteva quella «lingua nostra» in una ultima esecuzione solenne.

Vitale individua le componenti linguistiche e retoriche dell'edificio narrativo, perché se ne assimili tecnicamente il fascino, perché si comprenda se e come quello che l'autore volle «contendere al tempo, addurre da esso al mondo delle forme, non ripugnò tutto al potere dell'arte». 

Isabella Gualandri

\author{
Il Tasso e Lucano \\ Dissonanze E CONSONANZE
}

La presenza nella Gerusalemme Liberata di numerosissimi echi dai grandi classici latini - Virgilio anzitutto, ma anche Ovidio, Orazio, Lucano, Stazio, Silio Italico, spesso difficili da riconoscere perché mediati dalle riprese già fattene nella tradizione poetica italiana - è stata segnalata fin dai primi commenti al poema, e ripetutamente e sparsamente analizzata nella ricchissima bibliografia dedicata al Tasso: temo quindi che appaia quasi come un gesto di hybris il fatto che chi, come me, si è sempre occupata di tutt'altro, pretenda di intervenire su un argomento così vasto e complesso, per di più di fronte a studiosi - primo fra tutti Maurizio Vitale - che hanno con l'autore una dimestichezza di gran lunga maggiore della mia. Ma confido che, sia pure in misura minima, ritornare sul problema consenta di aggiungere qualche piccolo tassello al grande mosaico. L'ampiezza del tema richiede di delimitare drasticamente il campo: mi concentrerò sull'emergere di tratti propri della poesia di Lucano nella Liberata.

Latteggiamento del Tasso nei riguardi di Lucano rivela a mio avviso un certo contrasto fra teoria e pratica.

Nei Discorsi dell'arte poetica e in particolare sopra il poema eroico, dedicati a Scipione Gonzaga e pubblicati a Venezia nel I 587 (ma iniziati nel I562), e alcuni anni dopo (I594) nei Discorsi del poema eroico, indirizzati al cardinale Aldobrandini, che ne riprendono ed elaborano i temi, il Tasso, riflettendo sulla materia di cui deve trattare il poema eroico, si sofferma, com'è noto, su tre punti essenziali. ${ }^{1}$ Osserva anzi-

${ }^{1}$ Torquato Tasso, Scritti sull'arte poetica, a cura di Ettore Mazzali, tomo I e 
tutto che la materia «o si finge... o si toglie da l'istorie»; ed è preferibile questa seconda soluzione che rispetta l'esigenza della verisimiglianza. ${ }^{2}$ Discutendo poi del «meraviglioso» egli critica quello legato alla mitologia pagana, ossia quello «che portan seco i Giovi e gli Apolli e gli altri numi de' Gentili», da lui giudicato "freddo ed insipido e di nessuna virtù». ${ }^{3}$ Bisogna quindi scegliere argomenti tratti «da istoria non gentile, ma cristiana od ebrea». ${ }^{4}$ Solo in questo caso è possibile conciliare meraviglioso e verisimile, presentando cioè interventi di Dio, angeli, demoni etc. o di "coloro a' quali da Dio o da' demoni è concessa questa podestà, i quali sono i santi, i maghi e le fate»..$^{5}$ Infine bisogna evitare di scegliere la storia di tempi molto lontani, ${ }^{6}$ che richiede si introducano particolari riguardanti costumi antichi (ad esempio sul modo di combattere e simili), il che produce un certo fastidio: e si ricorda Omero, i cui poemi, "come che divinissimi siano, paiono nondimeno rincrescevoli». D'altro lato non si possono unire storia antica e costumi nuovi; mentre a sua volta la scelta di un argomento di storia moderna, che evita questa difficoltà, toglie «quasi in tutto la licenza di fingere, la quale è necessarissima a i poeti, e particolarmente a gli epici».? Quindi bisogna fissarsi su tempi né troppo vicini né troppo remoti.

A chi legga queste pagine sembrerà subito di riconoscere la descrizione del Bellum Civile di Lucano: è impostato su un argomento storico, ma non di storia contemporanea

II, Torino, Einaudi, 1977.

2 Discorsi dell'arte poetica: Discorso primo, in Scritti sullarte poetica, t. I, p. 5 .

${ }^{3}$ Discorso primo, in Scritti sull'arte poetica, t. I, p. 8.

${ }^{4}$ Discorso primo, in Scritti sull'arte poetica, t. I, p. Iо.

${ }^{5}$ Discorso primo, in Scritti sull'arte poetica, t. I, p. 9.

${ }^{6}$ Discorso primo, in Scritti sull'arte poetica, t. I, pp. Iо- I 2.

${ }^{7}$ Discorso primo, in Scritti sull'arte poetica, t. I, p. I 2. 
al poeta, ${ }^{8}$ bensì precedente di un centinaio d'anni: il che consente l'invenzione di alcuni episodi, quali ad esempio la presenza di Cicerone sul campo di battaglia di Farsalo (7.62 sgg.), o la visita, in realtà mai avvenuta, di Cesare alle rovine di Troia (9.950 sgg.: un intenso brano sulla caduta fatale dei grandi imperi e delle città, che lascia traccia nel Tasso ${ }^{9}$ ). Inoltre il «meraviglioso» lucaneo non si affida agli dei della tradizione pagana, ma ad arti magiche, assimilabili a quelle che il Tasso collega ai demoni, quali la famosa scena di necromanzia del sesto libro in cui la maga Eritto, richiamando in vita un soldato caduto in combattimento, ne ottiene la rivelazione del tragico futuro che coinvolgerà Sesto Pompeo, la sua famiglia e Roma stessa. ${ }^{10}$

Ma se questi tratti sembrerebbero far supporre una evidente consonanza fra il Tasso e Lucano, Lucano viene poi assai raramente menzionato, per lo più per essere criticato: anzitutto (cito ancora dai Discorsi dell'arte poetica) per aver abbracciato "troppo ampia e copiosa materia»; dal momento che «non solo il conflitto di Farsaglia, come dinota il titolo, ma tutta la guerra civile fra Cesare e Pompeo... prese a trattare» ${ }^{11}$ (anche se il titolo attualmente in uso non è Pharsalia, ma Bellum civile): e in simili casi secondo il Tasso l'ampiezza della materia non lascia «luogo alcuno a l'invenzione ed a l'ingegno del poeta», anche se questo in verità non si può dire del poema lucaneo. Precisa inoltre il Tasso ${ }^{12}$ che se ritiene Lucano non

${ }^{8}$ Come non ne erano per altro mancati nel mondo latino: da Nevio ed Ennio a varie opere perdute del tempo delle guerre civili.

${ }^{9}$ Si veda infra.

${ }^{10}$ Bell. Civ. 6.4I 9 sgg.

${ }^{11}$ Discorso primo, in Scritti sull'arte poetica, t. I, p. I7. Alla critica a Lucano si associa quella a Silio Italico, che "tutta la seconda guerra Africana prese a trattare».

${ }^{12}$ Discorso secondo, in Scritti sull'arte poetica, t. I, p. 22. 
essere vero poeta, ciò non accade perché tratta avvenimenti veri. Non è poeta "perché talmente s'obliga a la verità de' particolari che non ha rispetto al verisimile in universale; e pur che narri le cose come sono state fatte, non si cura d'imitarle come dovriano essere state fatte». Si riconosce qui anzitutto, ovviamente, un'eco della teoria enunciata in Aristotele (Poet. $\mathrm{I} 45 \mathrm{Ib}$ ) a proposito della differenza fra lo storico e il poeta, che non risiede nell'uso della prosa o del verso, bensì nel fatto che lo storico "dice le cose che avvennero", il poeta dice le cose "quali potrebbero avvenire» (così va inteso il testo, come osserva Pierluigi Donini, che nella sua edizione della Poetica fa importanti precisazioni su questo passo). ${ }^{13} \mathrm{Ma}$ vi si coglie anche l'eco di critiche al Bellum Civile cristallizzatesi in età antica e ben rappresentate dal commento lapidario di Servio ad Aen. I.382 Lucanus... in numero poetarum esse non meruit quia videtur historiam composuisse non poema. ${ }^{14}$ Il giudizio è ribadito dal Tasso in modo più specifico nei Discorsi del poema eroico, ${ }^{15}$ dove viene chiamato in causa un elemento preciso, ossia l'ordine osservato da Lucano nella narrazione degli avvenimenti, che «non è l'ordine proprio de' poeti, ma l'ordine dritto e naturale in cui si narran le cose prima avvenute: e questo è commune a l'istorico». Ma nell'ordine che si conviene al poema eroico e che il Tasso chiama «artificioso» (o «perturbato» secondo la denominazione del Castelvetro), «alcune de le prime» - s'intende le prime cose - «deono essere dette primieramente, altre posposte, altre nel tempo presente deono esser tralasciate e riserbate a miglior occasione, come

\footnotetext{
${ }^{13}$ Aristotele, Poetica, a cura di Pierluigi Donini, Torino, Einaudi, 2008, p. 6I.

${ }^{14} \mathrm{Si}$ aggiunga Petronio, se lo specimen di Bellum Civile attribuito al personaggio di Eumolpo in Sat. I I9-I 24 deve esser visto in polemica con Lucano.

${ }_{15}$ Discorsi del poema eroico, libro III, in Scritti sull'arte poetica, t. II, pp. 220-23.
} 
insegna Orazio». ${ }^{16}$ Inoltre, non bisogna cominciare da un principio troppo remoto, e almeno in questo «merita maggior lode e minor riprensione Lucano di Stazio: perché l'uno, volendo cantar de le guerre civili, mette Cesare su 'l passo del Rubicone, dove, giudicato nemico dal Senato, fu costretto a far la guerra», mentre Stazio all'inizio della Tebaide comincia da troppo lontano, ossia «da le furie e da le maledizioni d'Edippo, che furono prima e fatal cagione de la discordia fra Eteocle e Polinice». "Nondimeno Lucano ancora avrebbe fatto meglio s'avesse posto Cesare in Tessaglia e collocatolo a fronte a Pompeo, e l'altre cose prima contenute avesse fatto raccontare».

Non manca infine un richiamo al noto parere di Quintiliano (Io.I.90), secondo il quale, dice il Tasso, Lucano «è più simile a l'oratore ch'al poeta» (per l'esattezza il classicheggiante e virgiliano Quintiliano, che sta passando in rassegna gli autori utili alla formazione dell'oratore, dice che Lucano, evidentemente per la forte impronta retorica, è più adatto ad essere imitato dagli oratori che dai poeti: magis oratoribus quam poetis imitandus).

Se però da simili affermazioni, di ordine teorico o comunque generale, si passa a esaminare come in concreto si comporti il Tasso nella Gerusalemme, si vedrà che, benché in misura infinitamente minore rispetto all'onnipresente Virgilio, anche Lucano ha lasciato una sua ben determinata impronta, e in qualche caso in momenti assai significativi del poema.

Mi riferisco anzitutto all'episodio più famoso, e da tempo ampiamente analizzato dagli studiosi: quello dedicato nel tre-

${ }^{16}$ Hor. ars 42-44 ordinis haec virtus erit et venus, aut ego fallor / ut iam nunc dicat iam nunc debentia dici, / pleraque differat et praesens in tempus omittat; / hoc amet, hoc spernat promissi carminis auctor. Al passo oraziano si richiama anche Servio, che, nella prefazione al commento all'Eneide, approva il fatto che Virgilio non cominci il suo racconto dalla caduta di Ilio. 
dicesimo canto della Gerusalemme alla selva di Saron, ${ }^{17}$ dove i Crociati hanno necessità di addentrarsi per ricavarne legna con cui preparare macchine da guerra, selva che gli incantamenti di Ismeno hanno trasformato in un impenetrabile luogo d'orrore. Benché la vicenda in sé - il ricorso alla selva per rifornirsi di legname - abbia un aggancio storico con quanto racconta la cronaca di Guglielmo di Tiro (8.6), principale fonte storica del Tasso, la situazione, com'è noto, coincide singolarmente con quella narrata da Lucano nel terzo libro del Bellum Civile, là dove Cesare, per le sue operazioni di assedio a Marsiglia, ordina ai soldati di abbattere i tronchi di un bosco vicino alla città, un bosco sacro, luogo di riti barbari, nei cui alberi, macchiati del sangue di sacrifici umani, sono rozzamente raffigurate sconosciute e paurose immagini di divinità (Bell. Civ. 3.399 sgg.). Ettore Paratore ha raccolto i molti elementi, in gran parte già segnalati dagli studiosi, per i quali il Tasso sembra essersi ispirato a questo passo lucaneo, ${ }^{18}$ sviluppandolo fino a creare un quadro terrificante in cui oscurità, putredine, suoni minacciosi, attestano la presenza di creature diaboliche che hanno trovato dimora negli alberi; non senza l'eco di un altro famoso brano del Bellum Civile, la fosca selva d'oltretomba del sesto libro, che fa da sfondo al rito di necromanzia della maga Eritto (Bell. Civ. 6.642 sgg.). Ma anche la sete che subito dopo, sempre nel tredicesimo canto, per l'eccessivo ardore del sole, tormenta i Crociati (XIII 72 sgg.),

${ }^{17}$ La descrizione della selva in Gerus. XIII I-5. Per il canto XIII si veda l'analisi di Marziano Guglielminetti, Canto XIII, in Franco Tomasi (a cura di) Lettura della Gerusalemme Liberata, Alessandria, Edizioni dell'Orso, 2005, pp. 3i 5-35.

${ }^{18}$ Ettore Paratore, De Lucano et Torquato Tasso, "Latinitas», I97I, pp. 23 sgg.; poi in Id., Dal Petrarca all'Alfieri. Saggi di letteratura comparata, Firenze, Olschki, I975, pp. 29I-3 I2. Per la descrizione lucanea del taglio degli alberi nella selva (Luc. 3.440-442) ripresa in Gerus. III 75-76, ma già fatta propria dall'Ariosto, vd. Guglielminetti, Canto XIII, p. 3 I 8. 
benché storicamente attestata da Guglielmo di Tiro (8.7), richiama la sete dei Pompeiani a Ilerda, assediati da Cesare che ha loro tagliato i rifornimenti di acqua (Bell. Civ. 4.292 sgg.). Del resto, il racconto dell'assedio di Marsiglia, nella parte del terzo libro che vien dopo l'episodio della selva e descrive la preparazione e l'uso delle macchine da guerra (torri, balliste, arieti) lascia tracce evidenti anche nel diciottesimo libro della Liberata, insieme con tratti della tendenza lucanea a soffermarsi con macabro compiacimento sulle descrizioni di stragi e massacri (come quello che accompagna, sempre nel terzo libro, la battaglia navale che si svolge davanti a Marsiglia, 3.656 sgg.: ma altri confronti si potrebbero istituire).

Una tale concentrazione di analogie ha fatto dire al Paratore che il tredicesimo e il diciottesimo canto della Gerusalemme si presentano quasi come florilegi lucanei. ${ }^{19}$

Che ciò accada in particolare nel tredicesimo canto a me sembra assai significativo, poiché si tratta di un canto centrale nell'economia del poema, come precisa il Tasso stesso in una famosa lettera del 27 aprile I 575 a Scipione Gonzaga; ${ }^{20}$ un canto che, per adoperare le parole del poeta, benché non sia a metà del «quanto», ossia dell'insieme dei venti libri della Gerusalemme, costituisce però «il mezzo... della favola», là dove cioè, dopo un crescendo di difficoltà e disavventure per i cristiani («son mal trattati nell'assalto; vi è ferito il capitano; è poi arsa la lor machina, ch'era quella che sola spaventava gli nemici; incantato il bosco, che non se ne possono far dell'altre; e sono in ultimo afflitti dall'ardore della stagione e dalla penuria dell'acque, e impediti d'ogni operazione») avviene una sorta di rivolgimento: scende la pioggia in seguito alle preghiere di Goffredo, e da questo momento la situazione volge al positivo.

\footnotetext{
${ }^{19}$ Paratore, De Lucano et Torquato Tasso, p. 3 I 2.

${ }^{20}$ Cfr. T. Tasso, Lettere poetiche, a c. di Carla Molinari, Parma, I995, pp. 45 sgg. (lettera VI).
} 
È stato efficacemente osservato da Ezio Raimondi che con questa asimmetria strutturale il Tasso in qualche modo si contrappone all'Eneide, che invece colloca proprio a metà, nel sesto libro, il racconto determinante della discesa di Enea all'Averno, quasi una iniziazione in cui l'eroe dalla visione dell'aldilà e dall'incontro con il padre trae la consapevolezza di ciò che l'attende e si stacca definitivamente dal suo passato, pronto alle nuove e risolutive vicende che caratterizzano la seconda parte del poema. ${ }^{21}$ Se è vero, come credo possibile, che «l'avventura dei crociati nella foresta della magia diabolica equivale a una discesa agli inferi, tanto più significativa allorché la si confronta con quella di Enea di cui vuol essere una trasposizione» (sono sempre parole di Ezio Raimondi), ${ }^{22}$ la presenza e la funzione di Lucano nell'ispirare un quadro così ossessivamente cupo e ostile in un episodio che fa da cardine nella struttura del poema, acquista, credo, un'importanza particolare. Non va dimenticato che era stato Lucano stesso, in primis, a contrapporsi a Virgilio, creando, con la descrizione del rito di necromanzia della maga Eritto, un equivalente della discesa di Enea all'Averno, e collocandolo proprio nel sesto libro del Bellum Civile, in allusiva corrispondenza numerica con il testo virgiliano, ma con asimmetria strutturale, almeno apparente, rispetto allo stesso, essendo il poema lucaneo, così come ci è giunto e come lo conosceva anche il Tasso, costituito di dieci libri (lasciamo da parte la questione su cui si sono concentrati successivamente gli studiosi di quale potesse essere il disegno complessivo nella mente dell'autore). ${ }^{23}$ Colori e asimmetria lucanei, quindi: in

${ }^{21}$ E. Raimondi, Poesia come retorica, Firenze, Olschki, I980, p. I02; E. Raimondi, Introduzione a T. Tasso, Gerusalemme Liberata, a cura di Bruno Maier, Milano, Rizzoli, $200 \mathrm{I}^{6}$, pp. xxxi sgg.

22 Raimondi, Introduzione, p. XLIII; cfr. anche Poesia come retorica, p. I02.

23 Com'è noto, partendo dalla constatazione che il poema sembra 
una sezione determinante del suo poema il «virgiliano» Tasso (tale appariva ad esempio al Marino, che qualificava invece sé stesso come ovidiano ${ }^{24}$ si ispirava a Lucano per discostarsi significativamente dal modello prediletto.

Non mancano inoltre momenti in cui, anche per semplici schemi narrativi e impostazioni strutturali, nella Gerusalemme traspare in filigrana la presenza di Lucano. È noto ad esempio che il personaggio del pio Goffredo in più di un caso è debitore al Cesare lucaneo, benché l' ethos dei due sia ben diverso. ${ }^{25}$ L'esempio più famoso è quello del discorso di Goffredo alle sue truppe ( $\mathrm{xx}$ I4-19), che riprende assai da vicino il discorso di Cesare ai suoi, prima dello scontro di Farsalo (Bell. Civ. 7.250-336), ripetendone non solo i temi (la certezza della vittoria; la svalutazione dei nemici; la conoscenza che il comandante ha, per lunga consuetudine, di tutti i suoi soldati), ma anche le movenze espressive. ${ }^{26}$ Soltanto, scompaiono nel di-

strutturato su gruppi di quattro libri, ciascuno concluso dalla morte di uno dei personaggi più significativi della guerra civile (Curione a fine del quarto; Pompeo a fine dell'ottavo) si ipotizza o una struttura in dodici libri (speculare all'Eneide e conclusa dalla morte di Catone) o una struttura in sedici libri (conclusa dalla morte di Cesare).

${ }^{24}$ Lo ricorda Paratore, De Lucano et Torquato Tasso, p. 29I.

${ }^{25}$ Che il pio Goffredo fosse modellato sul duro personaggio di Cesare era singolarità che colpiva gli studiosi: cfr. Stefano Verdino, Canto Xx, in Tomasi (a c. di) Lettura della Gerusalemme Liberata, p. 50I. Vd. anche David Quint, Epic and Empire. Politics and Generic from Virgil to Milton, Princeton N. J., Princeton University Press, I993, p. 2 I 6.

${ }^{26} \mathrm{Si}$ vedano i confronti indicati dai commentatori: Gerus. xx I4 "O de' nemici di Giesù flagello, / campo mio, domator de l'Oriente, / ecco l'ultimo giorno, ecco pur quello / che già tanto bramaste omai presente» e Lucano, Bell. Civ. 7.250 sgg. O domitor mundi, rerum fortuna mearum, I miles, adest totiens optatae copia pugnae. I Nil opus est votis...; Gerus. xx I6 "Quei che incontra verranci, uomini ignudi / fian per lo più, senza vigor, senz'arte, / che dal lor ozio o da i servili studi / sol violenza or allontana e parte» e Lucano 7.270 sgg. Grais delecta iuventus / gymnasiis aderit studioque ignava palaestrae / et vix arma ferens...; Gerus. Xx I 8 «Ma 
scorso di Goffredo, abbastanza ovviamente, le punte più aspre dell'allocuzione di Cesare, quelle che evidenziano la tremenda tragicità della guerra civile, come ad esempio l'invito ad uccidere impietosamente chi si troverà a combattere sull'altro fronte, fosse anche un congiunto o lo stesso padre. ${ }^{27}$ Di contro, il mesto discorso di Pompeo (Bell. Civ. 7.342 sgg.), che corrisponde a quello di Cesare - «mesto» lo definisce Luca$\mathrm{no}^{28}$ - con un intenso richiamo agli affetti più cari, invita a combattere come se si fosse sotto gli occhi delle madri, dei vecchi, dell'intero popolo di Roma che non vuol perdere la libertà. ${ }^{29}$ In quest'antitesi si ripete la contrapposizione fra i due personaggi splendidamente preannunciata nel primo libro del Bellum Civile attraverso due famose similitudini: Cesare

capitano i' son di gente eletta: / pugnammo un tempo e trionfammo insieme, / e poscia un tempo a mio voler l'ho retta. / Di chi di voi non so la patria o 'l seme? / quale spada m'è ignota? o qual saetta, / benché per l'aria ancor sospesa treme / non saprei dir se Franca o se d'Irlanda, / e quale a punto il braccio è che la manda?» e Lucano $7.285 \mathrm{sgg}$. Sed me fortuna meorum / commisit manibus, quorum me Gallia testem / tot fecit bellis. Cuius non militis ensem / agnoscam? caelumque tremens cum lancea transit, / dicere non fallar, quo sit vibrata lacerto. E le analogie continuano nei versi seguenti. - Che il Tasso, rispetto all'Ariosto, fosse «maggiore e più manifesto imitatore delle particolarità, percioché senza velo alcuno trapporta ciò che vuole imitare, usando assai forme di dire et elocuzioni latine, delle quali troppo evidentemente si serve» era parere di Giovan Battista Marino, La Sampogna, Lettera Iv, a cura di Vania De Maldé, Parma, Guanda - Fondazione Pietro Bembo, I993, p. 49.

${ }^{27}$ Bell. Civ. 7.320 sgg.: non vos pietatis imago / ulla nec adversa conspecti fronte parentes / commoveant: voltus gladio turbate verendos.

${ }^{28}$ A conclusione dell'allocuzione di Pompeo, che commuove i soldati, 7.382 s.: tam maesta locuti / voce ducis flagrant animi.

${ }^{29}$ Bell. Civ. 7.369 sgg.: credite pendentes e summis moenibus urbis / crinibus effusis hortari in proelia matres; / credite grandaevum vetitumque aetate senatum / arma sequi sacros pedibus prosternere canos, / atque ipsam domini metuentem occurrere Romam; / credite, qui nunc est populus populumque futurum / permixtas adferre preces: haec libera nasci, / haec volt turba mori. 
è come un fulmine che tutto travolge; Pompeo come un'antica quercia maestosa che non produce più foglie..$^{30}$ Non lo si dice, ma è ovvio, che il fulmine è destinato ad abbattersi sulla quercia: ma se Cesare è il vincitore, l'umana comprensione e la pietas di Lucano sono però dalla parte del vinto Pompeo. Nella Gerusalemme la situazione è naturalmente diversa e non consente questa sympatheia coi nemici; al discorso di Goffredo fa infatti riscontro nel campo avverso quello di Emireno (xx 24-27), più breve e non caratterizzato in senso lucaneo. ${ }^{31}$ $\mathrm{Ma}$ vorrei osservare che qualche tratto della malinconia del vinto Pompeo, a cui Lucano, descrivendone la fuga dal campo di battaglia, dedica nel settimo libro pagine tra le più belle del poema, ${ }^{32}$ si riverbera forse talora anche sugli infedeli sconfitti della Gerusalemme. Penso a Solimano, il quale (xx 73) mentre infuriava la lotta sotto le mura della città,
salse in cima a la torre ad un balcone
e mirò, benché lunge, il fer Soldano:
mirò, quasi in teatro od in agone,
l'aspra tragedia dello stato umano:
i vari assalti e 'l fero orror di morte,
e i gran giochi del caso e de la sorte.

Qui io riconosco Pompeo (Bell. Civ. 7.650 sgg. ${ }^{33}$ ) che, nel

\footnotetext{
${ }^{30}$ Cfr. Bell. Civ. I.I 35 -I 43 per Pompeo; I. I 5 I-I 57 per Cesare.

31 Sui due discorsi cfr. S. Verdino, Canto Xx, pp. 500 sgg. Su Cesare che altrove affronta i soldati rivoltosi (Bell. Civ. 5.285 sgg.) e sull'influsso del "clima morale tormentato del poema lucaneo" sul Tasso, cfr. Franco Pignatti, Canto VIII, in Tomasi (a cura di) Lettura della Gerusalemme Liberata, pp. 20 I sgg.

32 Pompeo fugge dal campo di battaglia (7.677-7II), sacrificando così la sua fama di condottiero, nella speranza che questo possa abbreviare il combattimento e risparmiare alcune vite.

33 Stetit aggere campi, / eminus unde omnes sparsas per Thessala rura / aspiceret clades...
} 
mezzo della battaglia, salito su un'altura contempla come uno spettacolo (e il tema dello «spettacolo» è stato studiato quale componente significativa del Bellum Civile) ${ }^{34}$ la grande strage che coinvolge genti di ogni parte della terra, in funere mundi, come lo definisce Lucano. ${ }^{35}$

Anche il decimo libro di Lucano, dedicato al soggiorno di Cesare in Egitto, al suo incontro con Cleopatra, alla descrizione della reggia di Cleopatra e del lussuoso banchetto da lei offerto a Cesare (Bell. Civ. Io. I I I-I7I) offre immagini e colori che si ritrovano nel Tasso, ad esempio nella descrizione del palazzo d'Armida (xvi 2.3 sgg.). E Armida stessa serba taluni tratti di Cleopatra: allo stesso modo che Cleopatra, privata del regno, facendo affidamento sulla sua bellezza, si reca da Cesare narrandogli come ha perso il potere, e concedendogli i suoi favori ne ottiene la protezione (Bell. Civ. Io.Io4 sgg.), simulatum compta dolorem (83), ${ }^{36}$ "adorna di un finto dolore», così a Goffredo si presenta a chiedere soccorso Armida (IV 39 sgg.) con il suo racconto di sventure, ostentando anch'essa un «finto dolor» (IV 77) che commuove molti fino alle lacrime, ma non convince il pio capitano (Iv 68), il quale cortesemente risponde che le porterà aiuto, ma solo dopo aver concluso la sua impresa. Di più: in Lucano la sosta di Cesare in Egitto, nell'ambiente fastoso e vizioso di Cleopatra, arreca di fatto danno al suo esercito consentendo ai pompeiani di riorganizzarsi, ${ }^{37}$ così come nella Gerusalemme il soggiorno di

\footnotetext{
${ }^{34}$ Matthew Leigh, Lucan. Spectacle and Engagement, Oxford, Clarendon Press, I997.

${ }^{35} 6 \mathrm{I} 7 \mathrm{sgg}$. impendisse pudet lacrimas in funere mundi / mortibus innumeris, ac singula fata sequentem / quaerere...

${ }^{36} 82$ sgg. quem formae confisa suae Cleopatra sine ullis / tristis adit lacrimis, simulatum compta dolorem / qua decuit, veluti laceros dispersa capillos etc.

${ }^{37}$ Cfr. Bell. Civ. I 0.77 sgg., Cesare oblitus Magni tibi Iulia, fratres / obscaena de matre dedit, partesque fugatas / passus in extremis Libyae coalescere regnis / tempora Niliaco turpis dependit amori, I dum donare Pharon, dum
} 
Rinaldo presso Armida è rovinoso per i Crociati. Del resto un collegamento tra Armida e Cleopatra è istituito dallo stesso Tasso, allorché rappresenta Armida che, scesa in battaglia con gli altri, fugge nel mezzo del combattimento, allo stesso modo che Cleopatra aveva abbandonato Antonio durante lo scontro di Azio: ${ }^{38}$ ma questa volta il modello che sta alle spalle della comparazione non è Lucano, bensì Virgilio, nella descrizione della battaglia Azio che appare sullo scudo di Enea ${ }^{39}$, o forse Orazio, nella famosissima ode dedicata alla morte di Cleopatra, nunc est bibendum. ${ }^{40}$

Ma a mio parere la presenza di Lucano si avverte anche in un motivo topico, di antica tradizione epica quale la rassegna delle truppe di Goffredo nel canto primo, ottave 37-44, che descrive lungamente i suoi guerrieri, principalmente Franchi. È mia impressione che il Tasso abbia avuto in mente l'insolito catalogo che appare nel primo libro del Bellum Civile, dove Cesare, per invadere l'Italia, richiama i soldati romani stanziati in varie parti della Gallia per tenere a freno le diverse tribù. La rassegna, rovesciando per così dire il tema tradizionale non è, in effetti, un catalogo delle truppe di Cesare, bensì delle tribù galliche che rimarranno senza controllo, e che sono brevemente ricordate per le loro caratteristiche fisiche,

non sibi vincere mavolt (con l'allusione anche ai figli che Cesare ebbe da Cleopatra). Su Armida-Cleopatra cfr. anche Quint, Epic and Empire, p. 39.

38 Gerus. xx I I 8 «Tal Cleopatra al secolo vetusto / sola fuggia da la tenzon crudele, / lasciando incontra al fortunato Augusto / ne' maritimi rischi il suo fedele, / che per amor fatto a se stesso ingiusto / tosto seguì le solitarie vele». ${ }^{39}$ Aen. 8.707 sgg. ipsa videbatur ventis regina vocatis / vela dare et laxos iam iamque immittere funis. Sul tema cfr. Raimondi, Poesia come retorica, pp. I 98 sgg.; Arnaldo Soldani, Canto XVII, in Tomasi (a cura di) Lettura della Gerusalemme Liberata, p. 425, n. I7.

${ }^{40}$ Che la rappresenta inseguita da Ottaviano: Hor. carm. I.37.I 6 s. Caesar ab Italia volantem / remis adurgens. 
per il modo di combattere, per i luoghi da loro abitati. I quali luoghi - le diverse parti della Gallia - sono in generale sovrapponibili a quelli dei guerrieri Franchi, il che rende più facili le consonanze: ricordo i lunghi crini dei Franchi (Gerus. I 39.3 «sotto l'elmo premendo i lunghi crini») e quelli dei Liguri o dei Caìci in Lucano, tipici della Gallia Comata (Bell. Civ. I.442 et nunc tonse Ligur, quondam per colla decore I crinibus effusis toti praelate Comatae; 463 crinigeros Caycos); il color biondo della capigliatura dei Fiamminghi nel Tasso (Gerus. I 43.I «seguia la gente poi candida e bionda / che tra i Franchi e i Germani e 'l mar si giace») e di quella dei Ruteni in Lucano (Bell. Civ. I.402 flavi...Ruteni). Analogie abbastanza ovvie, si dirà: forse meno ovvio il cenno, nel Tasso, alla violenza dell'Oceano sulle coste olandesi, là dove esso voracemente (Gerus. I 43.5) «non pur le merci e i legni, / ma intere inghiotte le cittade e i regni», che sembra sviluppare e per così dire aggiornare un suggerimento lucaneo sulla forza delle maree atlantiche (Bell. Civ. I.409 sgg. quaque iacet litus dubium quod terra fretumque / vindicat alternis vicibus, cum funditur ingens / Oceanus vel cum refugis se fluctibus aufert). Ancor più significativo a mio avviso il tratto che nella Gerusalemme si riferisce ai guerrieri di Guelfo, il quale, oltre a regnare per diritto ereditario sulla Carinzia e sui territori un tempo occupati da Svevi e Reti, conduce con sé, dalle terre da lui conquistate, gente coraggiosa (Gerus. I 42.3) «che prende a scherno / d'andar contra la morte, ov'ei comandi; / usa a temprar ne' caldi alberghi il verno, / e celebrar con lieti inviti i prandi». Questo disprezzo della morte, che nel Tasso è legato alla fedeltà al proprio comandante, si trova anche nella rassegna lucanea, ma viene attribuito alla particolare concezione che della morte hanno i Druidi, convinti che le anime si reincarnino immediatamente in un altro corpo, e che si muoia solo per rivivere: onde il commento del poeta (Bell. Civ. I.458-60) sui populi ... felices errore suo, quos ille timorum 
/ maximus haud urguet, leti metus. Ed è altra coincidenza significativa che in Lucano tali popoli siano definiti come nordici (I.458 quos despicit Arctos), così come uno scenario nordico è evocato dal Tasso: "gente... usa a temprar ne' caldi alberghi il verno, / e celebrar con lieti inviti i prandi». ${ }^{41}$

Una sottile consonanza con Lucano credo si possa inaspettatamente avvertire nel quindicesimo canto della Gerusalem$m e$, nell'episodio del viaggio di Carlo e Ubaldo, sulla imbarcazione guidata dalla Fortuna, da Ascalona verso Occidente fino alle isole Fortunate (Canarie) dove ha dimora Armida e dove è trattenuto Rinaldo. ${ }^{42}$ Penso all'ottavo libro del Bellum Civile, là dove Pompeo, in fuga dopo la rotta di Farsalo, giunge dapprima a Lesbo dove si trovava la moglie Cornelia (8.40 sgg.) e decide di muovere di lì verso l'Egitto, per rifugiarsi presso la corte di Tolomeo. ${ }^{43}$ Naturalmente le rotte dei due viaggi coincidono solo per un breve segmento, poiché quello di Pompeo muove da una parte più orientale del Mediterraneo e si conclude in Egitto, laddove quello di Carlo e Ubaldo ha inizio poco prima dell'Egitto, e punta poi verso un più lontano e misterioso occidente, oltre le colonne d'Ercole. Il modo di procedere è, ovviamente, analogo, attraverso l'enumerazione e (soprattutto nel Tasso) la descrizione dei vari luoghi che s'incontrano (fiumi, città, porti e simili): ma mi colpisce la presenza, nel viaggio di Carlo e Ubaldo, di un particolare che mi sembra abbia una precisa funzione allusiva a Lucano.

Nel Bellum Civile Pompeo (8.243 sgg.) tocca una serie di città dell'Asia Minore (Efeso, Colofone, Samo, Cos, Cnido,

41 Un tocco che sembra riecheggiare certi tratti della descrizione dei costumi dei Germani fatta da Tacito, ad es. Germ. 22 (i Germani che si lavano con acqua spesso calda, ut apud quos plurimum hiems occupat, e poi siedono a pranzo).

42 Gerus. Xv 8 sgg.

${ }^{43}$ Cfr. in 8. 44I-455 la proposta in questo senso di Lentulo, che viene accolta da Pompeo. 
Rodi, Telmesso, la Panfilia e la Cilicia, minuziosamente ricordate) per volgere poi (456) verso Cipro, l'Egitto, il delta del Nilo e nei suoi pressi (470) verso la zona del monte Casio, dove si trova il re Tolomeo. Proprio qui (539 sgg. perfida qua tellus Casiis excurrit harenis / et vada testantur iunctas Aegyptia Syrtes) Achilla, il comandante dell'esercito egiziano, scelto come sicario, uccide Pompeo e lo fa decapitare. E qui (729 sgg.) un personaggio per altro sconosciuto, Cordo, ne troverà il cadavere e provvederà ad una pietosa quanto improvvisata sepoltura, segnata da una semplice scritta, hic situs est Magnus (793). Ma, commenta il poeta, nessun visitatore del Nilo, nessun mercante che passi in queste zone mancherà di fare una deviazione verso il venerabile saxum di quella tomba, che sarà venerato più dello stesso Giove che sul monte Casio ha un santuario. ${ }^{44}$ Lassenza di una tomba sontuosa paradossalmente ricorderà Pompeo ai posteri più che un solenne e ricco monumento. ${ }^{45}$

É significativo, a mio avviso, che dopo che Carlo e Ubaldo hanno costeggiato Ascalona e Gaza (xv Io), il Tasso faccia loro apparire la visione del Casio (di cui per altro si tace il nome), e che il monte sia collegato al ricordo di Pompeo: (xv I $55 \mathrm{sgg}$.) «non lunge un monte poi le si scopriva», (s'intende alla barca della Fortuna) «che sporge sovra 'l mar la chioma altera / e i piè si lava ne l'instabil onde / che l'ossa di Pompeo nel grembo asconde». Sembra una sorta di omaggio a Lucano, volto quasi a confermare la persistenza della fama di quell'umile sepolcro, e a sfatare quanto Lucano mestamente afferma a conclusione del libro ottavo, profetizzando un futuro lon-

\footnotetext{
${ }^{44}$ Bell. Civ. 8.85 I sgg.: nam quis ad exustam Cancro torrente Syenen / ibit et imbrifera siccas sub Pliade Thebas / spectator Nili, quis rubri stagna profundi I aut Arabum portus mercis mutator Eoae, / Magne, petet, quem non tumuli venerabile saxum / et cinis in summis forsan turbatus harenis / avertet manesque tuos placare iubebit / et Casio praeferre Iovi?

${ }^{45}$ Bell. Civ. 8.858 sgg.: Nil ista nocebunt/ famae busta tuae: templis auroque sepultus / vilior umbra fores.
} 
tano in cui la modesta tomba di Pompeo scomparirà e la sua morte in Egitto sarà considerata favola mendace: ${ }^{46}$ il Tasso sembra voler dimostrare che non è così.

Mi chiedo infine se il particolare stesso che l'imbarcazione di Carlo e Ubaldo sia guidata dalla Fortuna (un tema, com'è noto, considerato poco ortodosso nell'ambiente del Tasso, e che non a caso verrà soppresso nella Conquistata) non possa essere stato suggerito proprio da Lucano, che in tutto l'ottavo libro menziona la Fortuna con frequenza quasi ossessiva (es. 86; 206; 27I; 3I 4; 335; 427; 486; 6I 5; 600; 70I; 767; 793; $86 \mathrm{I})$, in un punto, anzi (730), rivolgendosi a lei direttamente (730) e indicando Pompeo come sua creatura: Pompeius Fortuna tuus. ${ }^{47}$

Né mancano in questo episodio elementi che rinviano ad altre parti del poema lucaneo: il cenno a Faro, un tempo isola, ora congiunta al lido (Gerus. Xv I6 7 s.) «isola che già lunge I giacque dal lido, al lido or si congiunge», da confrontare con Bell. Civ. I0.509 sgg. nunc claustrum pelagi cepit Pharon. Insula quondam / in medio stetit illa maris sub tempore vatis / Proteos, ut nunc est Pellaeis proxima muris; o la visione dei resti di Cartagine, che evoca il tema della morte delle città: "Giace l'alta Cartago: a pena i segni / de l'alte sue ruine il lido serba. / Muoiono le città, muoiono i regni, / copre i fasti e le pompe arena ed erba, / e l'uom d'esser mortal par che si sdegni» (Gerus. XV 20, I-5): un tratto in cui, accanto a un passo del Sannazaro, ${ }^{48}$ è stato richiamato ${ }^{49}$ Lucano e il ricordo dell'epi-

${ }^{46}$ Bell. Civ. 8.867 sgg. pulveris exigui sparget non longa vetustas / congeriem, bustumque cadet, mortisque peribunt / argumenta tuae. Veniet felicior aetas, I qua sit nulla fides saxum monstrantibus illud; / atque erit Aegyptus populis fortasse nepotum / tam mendax Magni tumulo quam Creta Tonantis.

${ }^{47}$ Pompeo a sua volta così l'apostrofava in 7.665 s.: omnia quid laceras? quid perdere cuncta laboras?l iam nihil est, Fortuna, meum.

${ }^{48}$ De partu virginis 2.2 I 4-I9.

49 Bruno Basile, Il tempo e la memoria. Studi di critica testuale, Modena, 
sodio della visita di Cesare alle rovine di Troia nel nono libro, con la desolata visione dei suoi resti irriconoscibili e soffocati dalla vegetazione: Bell. Civ. 9.966-69 iam silvae steriles et putres robore trunci / Assaraci pressere domos et templa deorum / iam lassa radice tenent, ac tota teguntur / Pergama dumetis, etiam periere ruinae.

Ma, di là da tutti questi particolari, l'esempio a mio avviso emblematico del modo in cui si rivela la consonanza del Tasso con Lucano si trova là dove il suo ricordo si inserisce, con tratti di alto pathos, a trasformare il più sfumato tessuto espressivo di Virgilio.

Mi riferisco all'episodio della morte di Lesbino, il delicato giovinetto paggio di Solimano, che gioiosamente si lancia nel combattimento (Ix 83) ed è ucciso da Argillano, prima che il Soldano possa portargli aiuto: (Ix 85.7 sgg.) troppo tardi Solimano giunge e

vede, ahi dolor, giacerne ucciso

il suo Lesbin, quasi bel fior succiso.

$\mathrm{E}$ in atto sì gentil languir tremanti gli occhi, e cader su 'l tergo il collo mira; così vago è il pallore, e da' sembianti di morte una pietà sì dolce spira, ch'ammollì il cor che fu dur marmo inanti, e il pianto scaturì di mezzo a l'ira.

I particolari, il paragone col fiore, il collo che si abbandona inerte rimandano com'è noto, anche con corrispondenze precise, alla descrizione della morte di Eurialo in Aen. 9.433:

volvitur Euryalus leto, pulchrosque per artus it cruor inque umeros cervix conlapsa recumbit: purpureus veluti cum flos succisus aratro

Mucchi, I996, p. I 24. 
languescit moriens, lassove papavera collo demisere caput pluvia cum forte gravantur;

e ricordano la commozione e il gemito di Enea, turbato alla vista del morente Lauso, da lui ucciso: At vero ut vultum vidit morientis et ora, / ora modis Anchisiades pallentia miris, I ingemuit graviter miserans, etc. (Aen. I0.82 I-23).50

A questa vista Solimano piange; e spiccatamente lucaneo è l'intenso intervento del poeta (7-8) «Tu piangi, Soliman? tu, che destrutto / mirasti il regno tuo co 'l ciglio asciutto?». Allo stesso modo Cesare, narra Lucano nel nono libro, che con volto impassibile aveva calpestato i corpi dei senatori e senza una lacrima aveva guardato la strage nel campo di battaglia di Farsalo, non può frenare il pianto davanti al capo mozzato di Pompeo (Bell. Civ. 9.1043-46 qui duro membra senatus / calcarat vultu, qui sicco lumine campos / viderat Emathios, uni tibi Magne negare / non audet gemitus). È soprattutto significativa la movenza «tu piangi Soliman?», per quel modo di interpellare direttamente il personaggio, che, sostanzialmente estraneo a Virgilio ${ }^{51}$ è così comune invece in Lucano, il quale proprio qui - I046 sgg. - si rivolge a sua volta direttamente e lungamente a Cesare (buncine tu Caesar scelerato Marte petisti I qui tibi flendus erat? etc.). Ma Lucano lo fa per commentare sarcasticamente quelle lacrime che considera false. Un aspetto, quest'ultimo, che il Tasso preferisce ignorare, ${ }^{52}$ il che

\footnotetext{
${ }^{50}$ Sul tema, con i possibili richiami a figure di giovinetti in Virgilio, Stazio e nella tradizione rinascimentale, cfr. Roberto Gigliucci, Canto IX, in Tomasi (a cura di) Lettura della Gerusalemme Liberata, pp. 235 sgg.

${ }^{51}$ Il quale notoriamente interviene a commentare in prima persona solo in un luogo dell'Eneide, appunto nell'episodio di Eurialo e Niso, 9.446 sgg., per ricordare che grazie alla sua poesia non saranno dimenticati.

52 Tant'è vero che anche altrove, in un'epistola consolatoria (ep. 749, del I 587, a Dorotea Geremia negli Albizi, per la morte del marito) in cui elenca «eroi e re e capitani grandissimi, che hanno accresciuta quasi
} 
gli consente di recepire il motivo lucaneo spogliandolo della connotazione negativa per coglierne, con la propria sensibilità, la potenzialità affettiva: di fronte alla morte di un adolescente, dove un sentimento di stupore e pietas accompagna ancora una volta quel Solimano, altrove descritto come un lupo ingordo, ${ }^{53} \mathrm{di}$ demoniaca ferocia. ${ }^{54}$

Demetrio Falereo, nel suo De elocutione, che il Tasso conosceva nella traduzione latina attraverso i commentarii del Vettori ( 1562 ), ${ }^{55}$ descrive lo stile caratterizzato da deinotes, ossia gravitas, una qualità che il Tasso considera necessaria al poema eroico, come quello che richiede qualcosa di "veemente", "spezzato", ed è "simile a chi ferisce da vicino" (vehemens quiddam et concisum, et sane similis est ei qui hostem feriat cominus). Quanto questo richiami le caratteristiche dello stile lucaneo può esser dimostrato dal sintetico epigramma anonimo presente, a mo' di autoepitafio di Lucano, in vari manoscritti e nelle edizioni antiche del Bellum Civile, ma ora in genere dimenticato, in cui vien fatto parlare il poeta stesso: Corduba me genuit, rapuit Nero: proelia dixi, / quae gessere pares, hinc socer, inde gener. / Continuo numquam direxi carmina ductu,

dignità e riputazione al pianto ed a le lagrime», pone sullo stesso piano il pianto di Priamo per la morte di Ettore, di Enea per Anchise, di Achille per Patroclo, di Alessandro per Dario, di Annibale per Marcello, di Cesare per Pompeo. Cfr. T. Tasso, Epistolario. Con prefazione di Scipio Slataper, Lanciano, Carabba, I 9I 2, voll. 2, vol. II, pp. 35 sgg.

53 Gerus. x 2 "Come dal chiuso ovil cacciato viene / lupo talor che fugge e si nasconde, / che, se ben del gran ventre omai ripiene / ha l'ingorde voragini e profonde, / avido pur di sangue anco fuor tiene / la lingua e 'l sugge da le labra immonde, / tale ei se'n gia dopo il sanguigno strazio, / de la sua cupa fame anco non sazio».

${ }^{54}$ Gerus. IX 3 «quel Soliman di cui non fu tra quanti / ha Dio rubelli, uom più feroce allora, / né se per nova ingiuria i suoi giganti / rinnovasse la terra, anco vi fora".

${ }^{55}$ Cfr. Raimondi, Poesia come retorica, pp. 26 sgg. 
I quae tractim serpant: plus mihi comma placet. / Fulminis in morem, quae sint miranda, citentur: / haec vere sapiet dictio, quae feriat. ${ }^{56}$

I versi $3 \mathrm{sgg}$. definiscono con sintetica efficacia le tendenze dello stile di Lucano: "Non ho mai composto versi che fluissero in maniera continua e sinuosa: li preferisco spezzati. Ciò che si vuole susciti meraviglia, deve essere espresso alla maniera del fulmine. Sarà veramente efficace l'espressione che saprà ferire».

È l'immagine di una tensione espressiva da tutti riconosciuta a Lucano, che lo differenzia dal classicismo virgiliano per una costante tendenza al pathos e al sublime, ${ }^{57}$ in cui si colgono molti elementi comuni alle tragedie di Seneca, che hanno fatto parlare di «barocco» e di «manierismo».

E mi piace a questo proposito citare le parole con cui Maurizio Vitale, a conclusione del suo fondamentale lavoro sul Tasso epico, così sintetizza la ratio linguistica della Liberata: «i risultati artistici rendono, nel loro insieme, il poema singolare e affascinante e, concretando il passaggio dal classicismo rinascimentale, con la sua lingua regolata, e dal classicismo naturalistico tosco-fiorentino, con la sua lingua 'propria', al manierismo eletto, si pongono come iniziale modello della poesia barocca, a principiare dal Marino». ${ }^{58}$

Il Tasso si colloca, quindi, rispetto al classicismo rinascimentale, esattamente come Lucano rispetto al classicismo virgiliano: e in questo egli poteva avvertire una profonda sintonia con l'antico poeta.

\footnotetext{
${ }^{56}$ Anthologia Latina 668 Riese.

${ }^{57}$ Si ricordi che per Quintiliano (IO.I.90) Lucano era ardens et concitatus.

${ }^{58}$ Maurizio Vitale, L'officina linguistica del Tasso, Milano, LED, 2007, voll. 2, vol. II, p. 863 .
} 

Susanna Peyronel Rambaldi

\author{
ESULI ITALIANI «RELIGIONIS CAUSA» \\ E QUESTIONE DELLA LINGUA
}

1. Nel suo testo fondamentale su La questione della lingua, recentemente definito «la nostra bibbia per la questione della lingua», ${ }^{1}$ ma anche in numerosi altri studi, Maurizio Vitale esamina, tra i protagonisti del grande dibattito che animò la scena culturale italiana del primo Cinquecento, anche alcune figure di intellettuali e letterati, contigui o addirittura al centro di accademie, di gruppi e di circoli, coinvolti nel confronto religioso di quei decenni e nelle proposte di riforma o di rinnovamento spirituale, che maturarono anche in Italia. Non pochi furono oggetto di sospetti, di accuse e di processi da parte dell'Inquisizione, riorganizzatasi fin dal I 542 sotto il pontificato di Paolo III. Si tratta di episodi isolati, eppure significativi.

Questo intervento si occuperà di alcuni di essi, come Alessandro Citolini o il più famoso Ludovico Castelvetro, che sospettati e inquisiti divennero esuli religionis causa. In nome di una nuova letteratura, parteciparono ambedue a uno sforzo di affermazione e di disciplinamento critico del volgare italiano, ma questa apertura al rinnovamento non si limitò all'àmbito letterario, estendendosi anche al più intimo e insieme pericoloso àmbito spirituale.

In Italia, nella prima metà del secolo xvI, dissenso religioso e discussioni linguistiche si intrecciarono variamente, avendo

\footnotetext{
${ }^{1}$ Claudio Marazzini, Castelvetro linguista, in Ludovico Castelvetro. Letterati e grammatici nella crisi religiosa del Cinquecento, Atti della XIII giornata Luigi Firpo, Torino, 2 I-22 settembre 2006, a cura di Massimo Firpo Guido Mongini, Firenze, Olschki, 2008, p. I93; Maurizio Vitale, La questione della lingua, Palermo, Palumbo, $1984^{2}$.
} 
a volte i medesimi protagonisti. In una celebre pagina sulla letteratura italiana nell'età del Concilio di Trento, già Carlo Dionisotti osservava che, pur essendo «difficile trapassare con sicura competenza dalla storia letteraria del Cinquecento alla storia politica e religiosa, e viceversa", sarebbe stato utile riavvicinare alle "divergenze teologiche e politiche» anche altre divergenze, «linguistiche e letterarie», presenti nei medesimi uomini, appartenenti a una "vigorosa» società letteraria non necessariamente ribelle, ma neppure asservita, nei quali alternativamente quindi potevano prevalere esigenze «di libertà e di ordine, di eguaglianza e di qualificazione, di cordialità e di grandezza, di fedeltà al passato e di fedeltà a sé nel presente». ${ }^{2}$

Con l'affermarsi di una nuova lingua letteraria, tosco-fiorentina e culta, fu posto per tempo anche il problema delle traduzioni in volgare, e non solo per chi affermava essere il volgare "strumento idoneo all'espressione letteraria, anche più alta», ${ }^{3}$ dalla traduzione dei classici antichi, alle scienze $\mathrm{e}$ alla filosofia, ma anche per chi voleva rendere accessibili le Sacre Scritture, in un periodo, l'inizio del Cinquecento, in cui in tutta Italia erano impetuosi la curiosità e l'interesse per le novità religiose che venivano d'Oltralpe, cui s'intrecciavano sentimenti anticuriali e anticlericali già ampiamente radicati. ${ }^{4}$ Inizialmente fu soprattutto la lezione di Erasmo alla base di un'inquietudine culturale che caratterizzò molti ambienti umanistici, impegnati anche nella discussione sulla lingua e sull'importanza del volgare; dal caustico poligrafo Niccolò Franco, all'acuto linguista Claudio Tolomei o al grande letterato Benedetto Varchi, nella cui biblioteca era «massiccia la

\footnotetext{
${ }^{2}$ La letteratura italiana nell'età del Concilio di Trento, in Geografia e storia della letteratura italiana,Torino, Einaudi, I967, pp. 227-54.

${ }^{3}$ Vitale, La questione della lingua, p. 39.

${ }^{4}$ Massimo Firpo, "Disputar di cose pertinente alla fede». Studi sulla vita religiosa del Cinquecento italiano, Milano, Unicopli, 2003, pp. I2 I-40.
} 
presenza di Erasmo». 5 Le forme di erasmismo che si espressero in quei primi decenni del secolo furono varie. L'imitazione di Luciano da parte di Niccolò Franco nei Dialoghi piacevoli, ${ }^{6}$ ad esempio, portò lo sfortunato intellettuale, che pagò con la vita qualche decennio dopo la sua vena polemica e satirica contro il papato, ${ }^{7}$ a prediligere l'Erasmo "propagatore di un pensiero libero e indipendente», critico dell'ipocrisia del clero e delle degenerazioni del culto. ${ }^{8}$ Così nel Dialogo viII, di cui erano protagonisti «l'arte de i librari» e la stampa, e si pubblicizzavano le numerosissime traduzioni in volgare del tempo, esecrate dai «dotti», ma che i traduttori approntavano "per quegli che non intendono», Erasmo veniva difeso dalle censure non "perché ci sia scrupolo d'heresia», ma perché

il thedesco miracoloso t'ha concia in cordovana [ha ridicolizzato, fatto restare con un palmo di naso] tutta quella brigata [in Roma]. E perciò hanno pigliato in urto quel valent'uomo e non vogliono che in Roma compaia Erasmo. Ma non resta per questo ch'egli non si stampi e ristampi, non si venda e rivenda e non si legga e rilegga in ogni luogo.?

Nella feroce discussione culturale che attraversò il primo Cin-

\footnotetext{
${ }^{5}$ Sulla biblioteca del Varchi cfr. Massimo Firpo, Gli affreschi di Pontormo a San Lorenzo. Eresia, politica e cultura nella Firenze di Cosimo I, Torino, Einaudi 1997, pp. 254-57, in particolare p. 256.

${ }^{6}$ Emilio Mattioli, Luciano e l'umanesimo, Napoli, Istituto italiano per gli studi storici, I980, pp. I9I-97.

${ }^{7}$ Alberto Aubert, Paolo IV. Politica, Inquisizione e storiografia, Firenze, Le Lettere, I999, passim; Ottavia Niccoli, Rinascimento anticlericale, RomaBari, Laterza, 2005, pp. 258-73.

${ }^{8}$ Vedere Niccolò Franco, Dialoghi piacevoli, a cura di Franco Pignatti, Manziana (Roma), Vecchiarelli, 2003, Introduzione, pp. 28-29.

${ }^{9}$ Franco, Dialoghi piacevoli, pp. 303-04, Sulla difesa di Erasmo da parte del Franco nei Dialoghi piacevoli, cfr. anche Silvana Seidel Menchi, Erasmo in Italia. I 520-I 580, Torino, Bollati Boringhieri, I987, p. $28 \mathrm{I}$.
} 
quecento Franco si schierò dunque per gli «Erasmici» contro $\mathrm{i}$ «Ciceroniani», ${ }^{10}$

Nei medesimi anni il fiorentino Antonio Brucioli, invece, presentando il proprio volgarizzamento del Nuovo Testamento, riecheggiava l'Erasmo 'spirituale' quando scriveva che Cristo aveva voluto essere inteso «da tutte le lingue», ma soprattutto da «le semplici genti idiote». I volgarizzamenti erano offerti per «dilettare» gli animi, ma anche, come scriveva Erasmo, per un rinnovamento spirituale: andavano fatte traduzioni in volgare delle Sacre Scritture, perché anche le donnicciole, e «il tessitore, stando alla tela», e «il nocchiero intento al timone», potessero leggere l'Evangelo e consolare così «la sua fatica». ${ }^{11}$

Sovente i teologi, mettendo in relazione Umanesimo e Riforma, denunciarono l' «eloquenza degli eretici» come pericolosa, e l'uso della "grammatica» e della filologia riguardo alle Sacre Scritture come strumenti di sovversione religiosa. Ma la passione letteraria e religiosa nei confronti dei testi sacri e il cimentarsi nella loro lettura critica divennero negli anni Quaranta diffusissimi e vivi; addirittura una moda, che coinvolse la loquace e per molti versi litigiosa società letteraria, dall'Aretino allo stesso Bembo, che fu a contatto con «spirituali» poi sospettati di eterodossia, come i cardinali Contarini e Reginald Pole. Lo slancio divulgativo, culturale e religioso, che anche in Italia caratterizzò i primi decenni del Cinquecento, si intrecciò dunque con il problema del volgare e con la questione della lingua, e fu tra le cause dell'iniziale successo delle idee di riforma della Chiesa e di rinnovamento spirituale che si diffusero in quel periodo.

\footnotetext{
${ }^{10}$ Mattioli, Luciano e l'umanesimo, p. I9; Silvana Seidel Menchi, Alcuni atteggiamenti della cultura italiana di fronte ad Erasmo, in Eresia e Riforma nell'Italia del Cinquecento, Firenze-Chicago, Sansoni, Firenze-The Newberry Library, 1974, pp. 69-133.

${ }^{11}$ Seidel Menchi, Erasmo in Italia, p. 89.
} 
Non è un caso che tra i più autorevoli collaboratori di un grande editore come Aldo Manuzio ci fossero da un lato Erasmo e dall'altro Pietro Bembo: attraverso un editore, l'apertura culturale e pedagogica europea di un Erasmo poteva dunque coniugarsi con gli interessi linguistici e di rifondazione culturale di un Bembo. ${ }^{12}$ Tra gli anni Trenta e Quaranta, ad esempio, nella produzione di Niccolò Zoppino, che fu tra i più prolifici editori di questo periodo, accanto ai volgarizzamenti di classici si ebbero anche un impressionante numero di traduzioni italiane di Erasmo e addirittura i primi volgarizzamenti di testi di Lutero, editi senza il nome dell'autore. ${ }^{13} \mathrm{Ma}$ numerose furono anche le opere riguardanti il dibattito sulla lingua e su problemi grammaticali e ortografici, rilevanti anche per l'editoria: dalle Regole grammaticali della volgar lingua di Fortunio, pubblicate nel I 5 I6, al famoso Polito di Claudio Tolomei, edito da Zoppino nel I 53 I (la prima edizione romana era del 1525 ), un trattatello «scientifico di pronunzia e di grafia» che riformava l'ortografia e difendeva la «lingua toscana» come lingua che sembrava «di giorno in giorno farsi più bella», a beneficio "d'una università» di parlanti. ${ }^{14}$

Il senese Claudio Tolomei, definito da Maurizio Vitale «una delle menti linguistiche più acute e penetranti di tutto il Cinquecento", fu personaggio centrale nelle discussioni sulla lingua, anticipatore della linguistica scientifica e tra i primi a distinguere "la natura sociale e istituzionale della lingua dalla qualità letteraria che essa acquista ad opera degli scrittori, cioè l'uso della lingua dalla [sua] elaborazione letteraria» e arti-

${ }^{12}$ Luigi Severi, "Sitibondo nel stampar de’ libri». Niccolò Zoppino tra libro volgare, letteratura cortigiana e questione della lingua, Manziana (Roma), Vecchiarelli, 2009, pp. 34-35.

${ }^{13}$ Silvana Seidel Menchi, Le traduzioni italiane di Lutero nella prima metà del Cinquecento, "Rinascimento", I7 (I977), pp. 42-48.

${ }^{14}$ Vitale, La questione della lingua, p. 79; Severi, "Sitibondo nel stampar de” libri», pp. 3 I 2-I 8. 
stica. ${ }^{15}$ Personalità rappresentativa di una esuberante società letteraria, che condivise per un lungo periodo passioni culturali e interessi religiosi e che poi, proprio per motivi religiosi, si divise radicalmente e dolorosamente, Tolomei fu di salda ortodossia, in ottimi rapporti con la Chiesa di Clemente vII e di Paolo III, ma intrinseco, oltre che, come diremo, di Alessandro Citolini, dello «spirituale» Gasparo Contarini. Ammiratore della valdesiana Vittoria Colonna, fu in relazione con Pietro Carnesecchi, il protonotario apostolico giustiziato per eresia nel 1567 , e amico del senese Mino Celsi, che fuggirà dall'Italia religionis causa nel I $569 .{ }^{16}$

Letterato anzitutto e poi vescovo, fondatore dell'Accademia degli Intronati, esule politico da Siena, cortigiano a Roma nella corte fastosa del cardinale Ippolito de' Medici negli anni Venti e poi entrato nell'orbita dei Farnese, fu, come ha scritto Carlo Dionisotti, ${ }^{17}$ tra quegli «uomini nuovi» che avevano "voltato le spalle» alla tradizione umanistica latina, «decisi a produrre con tutta serietà, non per gioco, il loro sforzo nel solco fresco della tradizione italiana volgare», e devoti tanto al Bembo quanto a cardinali innovatori e poi sospettati di eterodossia come Contarini e Pole; «uomini nuovi» che "maneggiarono con uguale assiduità i testi di san Paolo e del Petrarca». Proprio al Tolomei il cardinale Gasparo Contarini dedicò, nel I 537 , una famosa e assai dibattuta lettera sulla predestinazione, sulla grazia e il libero arbitrio;

\footnotetext{
${ }^{15}$ Vitale, La questione della lingua, pp. 80-82. Sul Tolomei manca una biografia aggiornata; ancora utile Luigi Sbaragli, Claudio Tolomei, umanista senese del Cinquecento: la vita e le opere. Con prefazione di Guido Mazzoni, Siena, Accademia per le Arti e le Lettere, I939.

${ }^{16}$ Di queste relazioni sono testimonianza le lettere del Tolomei: De le lettere di M. Claudio Tolomei libri sette, con una breve dichiarazione in fine di tutto l'ordine de l'ortografia di questa opera, in Vinegia, appresso Gabriel Giolito de Ferrari, I 547.

${ }^{17}$ La letteratura italiana nell'età del Concilio di Trento, p. 233.
} 
argomenti divenuti brucianti dopo la protesta di Lutero. Nella città di Siena, infatti, a séguito di un violento scontro tra predicatori, era scoppiata una "gran confusione» nel popolo, e il Tolomei aveva chiesto che il cardinale si pronunciasse, perché la popolazione della sua città non giungesse «a subversione». La risposta del cardinale veneziano era stata, com'è noto, un lungo tentativo diplomatico di ritrovare una via media, che convincesse il Tolomei, tra predestinazione e libero arbitrio. ${ }^{18}$

Nel clima religioso incandescente dell'Italia di metà Cinquecento, tuttavia, queste discussioni erano ormai uscite dai circoli degli studenti universitari e dalle letture delle Lettere di san Paolo che si facevano tra dotti e, attraverso le parole dei predicatori, avevano raggiunto un vario pubblico cittadino.

Il letterato senese fu per altro tra i molti che apprezzarono le prediche del cappuccino Bernardino Ochino, allora conteso da tutte le città d'Italia, ammirandone la predicazione 'evangelica', perché, come si diceva, faceva "piangere le pietre». ${ }^{19}$ Quando, nel I 542, per sfuggire all'Inquisizione, l'Ochino andrà a Ginevra, anche Tolomei, come molti, resterà sbalordito: notizia «incredibile, e spaventosa», gli scriveva in una lettera famosa, che egli non riusciva a credere. Era infatti una "nuova» raccapricciante che un "huomo di molta prudenza, di bontà singolare, di somma religione» avesse potuto trasferirsi «dal campo de' catolici a gli alloggiamenti de' Luterani». Chiedeva all'Ochino addirittura di illuminarlo su una simile decisione, egli che predicava il nome di Cristo e la sua vera legge; egli che era «apprezzato, honorato, riverito e quasi [per] cosa divina adorato», considerato simile a un san Francesco.

\footnotetext{
${ }^{18}$ Giorgio Caravale, Sulle tracce dell'eresia. Ambrogio Catarino Politi (I484I 553), Firenze, Olschki, 2007, pp. 7I-78.

${ }^{19}$ Susanna Peyronel Rambaldi, Una gentildonna irrequieta. Giulia Gonzaga fra reti familiari e relazioni eterodosse, Roma, Viella, 20 I 2, pp. I I6-I 7.
} 
L'invito era a tornare indietro, per giustificarsi, per trovare perdono, perché in tempi tanto calamitosi l'unica salvezza stava nell'obbedienza alla Chiesa di Roma. ${ }^{20}$ Era la rottura, culturale e religiosa, di una societas comune, che Tolomei condivideva con molti, tra i quali anche Alessandro Citolini.

\section{Alessandro Citolini}

Alessandro Citolini di Serravalle (oggi nel comune di Vittorio Veneto) non solo fu uno di quegli «uomini nuovi» protagonisti del dibattito sulla lingua, ma anche tra coloro che si mossero tra umanesimo ed eterodossia. Allievo dell'irrequieto Giulio Camillo Delminio, famoso in tutta Europa per la sua grandiosa ambizione di racchiudere lo scibile umano in una "macchina» teatrale, ${ }^{21}$ il letterato di Serravalle, negli anni Trenta, seguì in Francia il maestro, che sperava nella liberalità di re Francesco i per la costruzione del suo teatro. Fu qui che probabilmente entrò in contatto con le prime idee eterodosse e, sebbene nulla si conosca di questo incontro con la cultura francese di corte - sono note tuttavia le simpatie filoriformate che in quegli anni caratterizzarono la cerchia della sorella del re, Margherita di Navarra ${ }^{22}$-, conobbe forse Johannes Sturm, amico di Melantone, che frequentava e stimava il Delminio, tanto da presentarlo nel I 533 al Butzer come «vir recondita eruditione, mirabili pietate». ${ }^{23}$ Certamente fu in contatto anche con la società colta francese, evocata dallo stesso Ca-

\footnotetext{
${ }^{20}$ De le lettere di M. Claudio Tolomei, cc. I89r-i 9 I $v$.

${ }^{21}$ Lina Bolzoni, Il teatro della memoria. Studi su Giulio Camillo Delminio, Padova, Liviana, I984; Cesare Vasoli, Su alcuni scritti «religiosi» di Giulio Camillo, in Filosofia e religione nella cultura del Rinascimento, Napoli, Guida, I988, pp. 279-32I.

22 Eleonora Belligni, Renata di Francia (I IIO-I 575). Un'eresia di corte, Torino, UTET, 20 I I.

${ }^{23}$ Giorgio Stabile, Camillo, Giulio, detto Delminio, in Dizionario biografico degli Italiani, Roma, Istituto della Enciclopedia Italiana, I974, vol. I7, p. 223.
} 
millo: da Guillaume du Bellay a Guillaume Budé, da Gérard Roussel, predicatore di Margherita di Navarra, a Lefevre d'Étaples. ${ }^{24}$ Di questa rete di relazioni tra Italia e Francia negli anni Trenta si sa ancora poco, ${ }^{25} \mathrm{ma}$ certo è che Citolini, ritornato a Serravalle, si fece notare per aver diffuso «de opinioni lutherane» nella sua città natale. ${ }^{26}$

Alla fine degli anni Trenta, malgrado sospetti e accuse, Citolini era a Roma, raccomandato ai letterati romani da Marcantonio Flaminio, anche lui di Serravalle; uno dei massimi esponenti degli «spirituali» italiani e autore occulto del Beneficio di Cristo, il più famoso libro eterodosso italiano. Fu quello un breve periodo durante il quale, con il cardinalato del Bembo nel I539, cultura e religione sembrarono coniugarsi e la riforma della Chiesa compiersi con il concorso degli studia humanitatis, non ostante l'espressa contrarietà di cardinali «intransigenti» come Gianpietro Carafa che, si dice, protestasse con il pontefice: «Padre santo, noi non habbi(a)mo in collegio di bisogno di huomini che sappiano fare i sonetti». ${ }^{27}$

Nella trionfante Roma farnesiana, all'inizio del papato di Paolo III, ${ }^{28}$ - quando ancora non era deflagrato nella Chiesa

${ }^{24}$ François Secret, Un témoignage oublié de Giulio Camillo Delminio sur la Renaissance en France, "Bibliothèque d'Humanisme et Renaissance», xxxiv (I972), pp. 275-78 e Vasoli, Su alcuni scritti «religiosi» di Giulio Camillo, pp. $283 \mathrm{sgg}$., che sottolinea le frequentazioni eterodosse del Delminio, soprattutto nei suoi soggiorni parigini.

25 Alcune osservazioni in Corrado Bologna, Lo "spirito" del "Cymbalum Mundi», in Le Cymbalum Mundi, Actes du Colloque de Rome (3-6 novembre 2000), a cura di Franco Giacone, Genève, Droz, 2003, pp. 207-I9. ${ }^{26}$ Adriano Prosperi, Un processo di eresia a Verona verso la metà del Cinquecento, "Quaderni Storici», I 5 (I 970), p. 785.

${ }^{27}$ Gigliola Fragnito, L'ultima visione: il congedo di Pietro Bembo, in Ead., In museo e in villa, Venezia, Arsenale, I988, p. 29.

${ }^{28}$ Massimo Firpo, "Navicula Petri". L'arte dei papi nel Cinquecento, RomaBari, Laterza, 2009, p. I 2 I. 
lo scontro tra intransigenti e spirituali e il cardinal Contarini veniva inviato, nel I 540, a Ratisbona nella speranza di un accordo con i protestanti, mentre cardinali moderati o riformatori speravano ancora di influenzare il promesso Concilio - Citolini frequentò il Tolomei e l'ambiente dei poeti che si raccoglievano attorno all'Accademia della Poesia nuova o della Virtù: Francesco Maria Molza, Annibal Caro, Luca Contile, Marcantonio Flaminio. «Famosi spiriti», «in tutte le scientie dottissimi» secondo il Contile, ${ }^{29}$ che condivisero passione poetica e ambiziosi progetti culturali, come quello del rinnovamento dell'architettura attraverso la traduzione in volgare e lo studio di Vitruvio, ma che in seguito si separarono sulle questioni religiose: gli uni per un'ordinata ortodossia, gli altri per un'inquieta eterodossia.

A Roma, probabilmente, Alessandro Citolini concepì la sua Lettera in difesa della lingua volgare, edita poi a Venezia nel I 540, ispirata alle discussioni con il Tolomei, testimoniate dal ben noto carteggio tra i due letterati. ${ }^{30}$ La Lettera si contrapponeva a umanisti come il friulano Romolo Amaseo e l'erudito Francesco Florido, elogiatori della nobiltà e universalità della lingua latina e denigratori del volgare. Per Citolini, invece, la lingua latina era "morta e sepolta ne' libri», mentre "la volgare è viva», una "vivente favella» parlata da tutti, uomini e donne, dotti e ignoranti, «e' come viva, cresce, genera, crea, produce, partorisce». ${ }^{31}$ Era una lingua, vera figliola e le-

\footnotetext{
${ }^{29}$ Alessandro Pastore, Marcantonio Flaminio. Fortune e sfortune di un chierico nell'Italia del Cinquecento, Milano, FrancoAngeli, I98 I, pp. 78 79 .

${ }^{30}$ Massimo Firpo, Citolini Alessandro, in Dizionario Biografico degli Italiani, Roma, Istituto della Enciclopedia Italiana, I982, vol. 26, pp. 39-46.

${ }^{31}$ La lettera d'Alessandro Citolini in difesa della lingua volgare; e i luoghi del medesimo con una lettera di Girolamo Ruscelli al Mutio, in difesa dell'uso delle Signorie, In Vinegia, Al segno del Pozzo, I 55 I, cc. 6r-v. Sulla Lettera in difesa della lingua volgare, cfr. Vitale, La questione della lingua, p. 50, p.
} 
gittima erede del latino, sempre rinnovata; lingua viva perché in bocca "de gli huomini, delle donne, de grandi, de piccioli, de dotti, de gl'ignoranti»; creativa, qualora anche non avesse alcuno scrittore al mondo, perché repertorio infinito e in continuo divenire, inventario aperto e duttile, ${ }^{32} \mathrm{da}$ chiunque in Italia comprensibile: «non è si basso Artefice, non è si vil Contadino; che di si fatti, e' così necessari vocaboli non sia meglio fornito, che tutta la lingua Latina». ${ }^{33}$ Nell'Italia delle "quattrocento Città», dei "Castelle senza numero, e villaggi senza fine», ${ }^{34}$ per essere intesi dai suoi numerosissimi abitanti bisognava dunque saper scrivere in volgare.

Non poteva, tuttavia, per Citolini, essere la sola lingua toscana il modello per dar vita a una lingua «tutta bella, tutta vaga, tutta ricca, e tutta perfetta», ${ }^{35}$ così come avevano fatto Petrarca e Boccaccio. L'immagine, molto suggestiva, della Toscana non come una prigione, ma come una «bella e spatiosa piazza dove tutti i nobili spirti d'Italia si riducono", si accompagnava a una fervida difesa della «libertà» letteraria, "tra tante ricchezze, e tanta abondanza di cose». ${ }^{36}$ Riprendendo il Tolomei, Citolini riaffermava che la lingua toscana era «pur senza dubbio la più bella, che ci sia», ${ }^{37}$ ma l'uso vivo della lingua stava forgiando un volgare perfetto non soltanto a «narrar favole, e amori», come sosteneva il Florido, ma anche a parlar «delle pietre», degli alberi, delle erbe, dei metalli, degli animali e dell'uomo, dell'economia e della politica, della guerra

\footnotetext{
I I 4; Anna Antonini, La riflessione linguistica di Alessandro Citolini, «Studi di Grammatica Italiana», I 8 (I999), pp. 257-82.

32 Ivi, p. 264.

${ }^{33}$ La lettera d'Alessandro Citolini in difesa della lingua volgare, c. $6 v$.

${ }^{34}$ Ivi, c. I $6 r$.

${ }^{35}$ Ivi, c. I $2 r$.

${ }^{36} \mathrm{Ivi}, \mathrm{cc} . \mathrm{I} 2 v$, I $4 r$.

${ }^{37}$ Ivi, c. I I $v$.
} 
e della pace, della morte e della vita, di agricoltura, delle arti e della medicina, e dunque anche, ed è questo l'aspetto che qui interessa, "della religione, della santità». ${ }^{38}$

L'elogio del volgare, dunque, si accompagnava all'entusiasmo di fronte all'ampliarsi degli spazi culturali, con l'esplosione editoriale che il nuovo secolo aveva veduto e la ricchezza infinita delle volgarizzazioni, dalla filosofia alla storia, dall'architettura all'astrologia. In ogni àmbito della cultura umana, in ogni aspetto dell'eredità dei classici greci e latini, spuntavano copiose le traduzioni in lingua volgare, e anche della «santa legge del nostro Dio insieme col suo commento, in lingua più vera, che non è la latina»:

noi habbiamo in questa lingua la Filosofia e' morale e' naturale, e sopra naturale; il che così a pieno non hebbero mai Latini [...]. Habiamo la santa legge dal nostro Dio insieme col suo commento, in lingua più vera, che non è la latina. Habbiamo assai trattati della scienza divina. Habbiamo assaissime historie. Habbiam più libri d'Architettura, habbiam l'Astrologia. Habbiam l'arte Poetica. Habbiam regole grammaticali, non pur di questa lingua, ma della Latina ancora. Habbiam diversi trattati di cose della Politica, e dell'Economica. Habbiam Comedie, Tragedie, Satire; e altre Poesie senza fine, e di molte più maniere che non ha la Latina, habbiam Livio, Cesare, Salustio, Terenzio [...] molte cose d'Aristotele [...] di Cicerone, di Boezio e di moltissimi altri e' Greci, e' Latini, trasportate in questa lingua. ${ }^{39}$

A questa ampia visione del volgare, Citolini accompagnava un sottile gusto dissacratorio, che rifletteva probabilmente i sentimenti anticlericali che a Serravalle, poco tempo prima, aveva condiviso con alcuni giovani del luogo, conosciuti perché «dicevano male de prelati e preti e frati, et religiosi» e dunque avversi «a li predicti capi chieregadi, che quelli have-

\footnotetext{
${ }^{38} \mathrm{Ivi}, \mathrm{c} . \mathrm{I} 7 v$

${ }^{39}$ Ivi, c. I $7 r$.
} 
vano paura de lori», come un inquisito per eresia confessò nel I 538 , accusando il Citolini di luteranesimo. ${ }^{40}$ La lingua latina si conviene ai dotti, sostenevano i suoi avversari. Ma quali dotti! irrideva il Citolini:

fusser dotti e intendenti come sono questi leggiadri preti, che vanno limosinando col breviario aperto in mano; per mostrar, che son dotti. De' quali ne potete veder le belle schiere per questa Città [Roma]. Oh non è da stimar più, senza comparazione, ogni picciol' homicciuolo ben' dotato dalla natura, quantunque ignudo di lettere latine, che centomila di questi tali di latino macchiati?

Ciò che, tuttavia, Citolini non poteva perdonare al proprio interlocutore era soprattutto che egli avesse detto male del Boccaccio, usando l'accusa «de non esser religioso; per haver detto mal de sacerdoti»:

$\mathrm{O}$ tristo che tu se Boccaccio, vatti confessa. - faceva il verso Citolini - Perché tu hai detto mal de sacerdoti non ti vogliam più leggere. Il tuo dir male delle persone religiose, ha fatto, che questa lingua non val più niente.

Boccaccio, invece, aveva soltanto mostrato «la corrotta, e abominevol vita de Preti; le incredibili, e infinite scelerataggini de Frati; la dishonesta, e sporca castità d'alcune Monache»; e dunque Citolini si domandava retoricamente: «chi fu manco religioso; o i sacerdoti, a fare il male, o il Boccaccio, a dirlo?».41 Egli era ancora lontano dall'immaginare quanto sarebbe divenuta inopportuna questa sua difesa del grande scrittore, che qualche anno dopo, nel I559, finì all'Indice. Il Decameron, com'è noto, fu sottoposto a una «rassettatura», cui l'insigne filologo e monaco benedettino Vincenzo Borghini si applicò volonterosamente ma inutilmente, dato che non soddi-

\footnotetext{
${ }^{40}$ Prosperi, Un processo di eresia a Verona, p. 785.

${ }^{41}$ La lettera d'Alessandro Citolini in difesa della lingua volgare, cc. I $6 v$, I $8 v$.
} 
sfece i censori. Il capolavoro venne in séguito spietatamente espurgato da Lionardo Salviati, divenendo questa versione, per molto tempo, l'unica legalmente concessa in lettura agli italiani. ${ }^{42}$

Tornato a Venezia, nel sensibile ambiente della capitale repubblicana, tra sentimenti anticlericali e ricerche di una pietà più autentica ed evangelica, Citolini fu legato a numerosi intellettuali veneti spiritualmente inquieti, come Orazio Brunetto e Giovanni Andrea Ugoni, Ludovico Domenichi e Jacopo Brocardo, una «consorteria spirituale» che abbracciava anche ambienti artistici, come ha mostrato Massimo Firpo, rappresentati dal pittore Lorenzo Lotto e dall'architetto Sebastiano Serlio. ${ }^{43}$ In anni più tardi, fu precettore dei figli di una gentildonna padovana, Caterina Sauli da Passano, la cui casa era divenuta negli anni Cinquanta un punto d'incontro di numerosi personaggi legati ai gruppi eterodossi di Venezia, Rovigo e Mantova (dove nel I 567 sarà processata per eresia). ${ }^{44}$ Condivise anche, e partecipò, al programma dell'Accademia padovana degli Infiammati, che faceva capo a Sperone Speroni, Benedetto Varchi, Daniele Barbaro, Bernardino Tomitano, e che aveva il proprio caposaldo nella difesa del volgare come lingua che poteva aprirsi anche ai generi alti, dalla filo-

\footnotetext{
${ }^{42}$ Rosa Mordenti, Le due censure: la collazione dei testi del Decameron "rassettati» da Vincenzio Borghini e Lionardo Salvati, in Le pouvoir et la plume. Incitation, contrôle et répression dans l'Italie du XVI.e siècle, Paris, Université de la Sorbonne Nouvelle, I982, pp. 253-74; Giuseppe Chiecchi - Luciano Troisio, Il Decameron sequestrato. Le tre edizioni censurate nel Cinquecento, Milano, Unicopli, I984; Le Annotazioni e $i$ discorsi sul "Decameron» del I 573 dei deputati fiorentini, a cura di Giuseppe Chiecchi, Roma-Padova, Antenore, 200 I.

${ }^{43}$ Massimo Firpo, Artisti, gioiellieri, eretici. Il mondo di Lorenzo Lotto tra Riforma e Controriforma, Roma-Bari, Laterza, pp. 97 sgg.

${ }^{44}$ Federica Ambrosini, L'eresia d'Isabella. Vita di Isabella da Passano signora della Frattina (I542-I60I), Milano, FrancoAngeli, 2005, passim.
} 
sofia, alle scienze. Così si rallegrava Citolini nella sua Lettera in difesa della lingua volgare: "Ogni dì qualche ornamento se le aggiugne. Ogni dì il numero de suoi amici s’aumenta. E ultimamente a difesa, et esaltazion sua la dottissima Academia de gl'Infiammati in Padova è suscitata».45

Soprattutto con lo Speroni Citolini fu in speciale sintonia. Ambedue polemizzavano con chi sosteneva che la lingua volgare non potesse parlare se non d'amore; ambedue manifestavano, come scrive Maurizio Vitale per lo Speroni, «una vivace coscienza democratica nell'àmbito della lingua e della cultura» che risultava "singolarissima e rivoluzionaria». ${ }^{46} \mathrm{Fa}-$ cendo parlare il Pomponazzi nel Dialogo delle lingue, «uno degli scritti più originali del lungo dibattito cinquecentesco» secondo Cesare Vasoli, ${ }^{47}$ lo Speroni, ad esempio, denunciava l'inutile «fatica dell'imparare» il latino e il greco per leggere gli autori classici, mentre "tutti i libri d'ogni scientia, quanti ne sono greci, et latini» avrebbero dovuto essere tradotti in volgare. I buoni studiosi sarebbero in questo modo divenuti assai più numerosi "che egli non è», mentre ora per apprendere quelle lingue "consumiamo la miglior parte de i nostri anni, la qual cosa non adivenia degli antichi». Provocatoriamente il Peretto sosteneva che

${ }^{45}$ La lettera d'Alessandro Citolini in difesa della lingua volgare, c. $23 v$.

${ }^{46}$ Vitale, La questione della lingua, p. 69. Sullo Speroni e «il clima ideologico d'avanguardia» che contraddistinse l'Accademia degli Infiammati e il gruppo di intellettuali che si radunarono attorno al letterato a Padova cfr. Francesco Bruni, Sperone Speroni e l'Accademia degli Infiammati, "Filologia e Letteratura», I3 (I967), pp. 24-7I.

${ }^{47}$ Cesare Vasoli, Sperone Speroni: la filosofia e la lingua. L'ombra' del Pomponazzi e un programma di volgarizzamento del sapere, in Il volgare come lingua di cultura dal Trecento al Cinquecento. Atti del Convegno internazionale, Mantova, I 8-20 ottobre 200 I, a cura di Arturo Calzona, F. Paolo Fiore, Alberto Tenenti, Cesare Vasoli, Firenze, Olschki, 2003, pp. 339-59, in particolare p. $34 \mathrm{I}$. 
i studi della lingua greca, et latina, sono causa della nostra ignorantia, che se il tempo, che in loro ci demo, si spendesse da noi in inparar philosophia, havrebbe forse la nostra novella età i platoni, et gli aristoteli dell'antica [...] tornati un'altra volta fanciulli altro non femo quindeci o venti anni anchora di nostra vita, che imparar a parlare chi latino, chi greco, et alcuni, se Dio vole, thoscano, li quali finiti, et finito inseme con loro, quel vigore, et quella prontezza d'animo, che naturalmente reca all'intelletto la gioventù, allhora procuremo di farsi philosophi, quando hoggimai non semo più atti alla speculazion delle cose. ${ }^{48}$

Era quanto già affermato dal Citolini nella sua Lettera sul volgare, non per la filosofia soltanto, ma anche per tutte le altre scienze: i giovani che andavano a studiare legge o medicina, scriveva, erano costretti a imparare il latino e il greco prima di aprire un solo libro delle loro discipline, cosicché «intorno a queste lingue si spende mezza questa vita così breve, prima che si incominci quell'arte così lunga. E poi si finisce la vita, prima che si arrivi a mezza l'arte». Era come se, scherzava il letterato, conosciuto come "huomo facetto e burlevole», ${ }^{49}$ chi volesse andare da Roma a Venezia passasse per l'Egitto e la Siria. Vedrebbe il mondo, ma non vedrebbe Venezia che è il suo «fine» e il suo «utile». 50

L'essenziale, come scrive Vasoli per quel che riguarda lo Speroni, stava "nello spostamento della quistione della lingua (e delle sue stesse radici storiche ed implicazioni politiche) dalla sua origine letteraria al problema effettuale della più vasta diffusione sociale del sapere». ${ }^{51}$ L'eredità dello Speroni venne dunque raccolta dall'Accademia della Fama, fondata da Federigo Badoer e operante tra il I557 e il I56I, cui col-

\footnotetext{
${ }^{48}$ Sperone Speroni, Dialogo delle lingue, a cura di Antonio Sorella, Pescara, Libreria dell'Università Editrice, I999, pp. I84-88.

${ }^{49}$ Ambrosini, L'eresia d'Isabella, p. I 8 I.

${ }^{50}$ La lettera d'Alessandro Citolini in difesa della lingua volgare, c. 2 I $r$.

${ }^{51}$ Vasoli, Sperone Speroni: la filosofia e la lingua, p. 347.
} 
laborò anche Citolini, e che aveva tra i vasti progetti culturali il volgarizzamento di opere filosofiche e scientifiche e la loro edizione, attraverso l'attività tipografica di Paolo Manuzio, figlio di Aldo. ${ }^{52}$

Nel I 565 , nonostante il persistente comportamento nicodemitico, il tribunale del Sant'Uffizio avviò a carico del Citolini un processo per eresia, che lo costrinse alla fuga, prima a Ginevra, poi a Strasburgo, e infine in Inghilterra. Nell'esilio come biglietto di presentazione e referenza presso la regina Elisabetta d'Inghilterra utilizzò la Tipocosmia, la sua opera più nota, composta negli anni Cinquanta e pubblicata a Venezia nel I 56I, ispirata certamente a Giulio Camillo Delminio e alla sua arte della memoria, ma anche, com'è noto, profondamente differente dal Teatro da questi immaginato, cosa, secondo il Citolini, "piu tosto da fanciulli, che da desiosi di sapere»..$^{53}$ Sorta di enciclopedia di tutte le arti liberali e rielaborazione del sapere a fini mnemonici, ma non macchina mnemotecnica artificiosa e meravigliosa come quella del Delminio, la Tipocosmia era piuttosto concepita come «un libbro di estrema grandezza», frutto maturo dell'arte della stampa, lontano da suggestioni ermetiche e cabalistiche. ${ }^{54}$ Citolini vi riversava tutta la sua sapienza lessicografica e cultura umanistica, facendo uso delle molteplici fonti di uno scrittorio in parte esibito, in parte occultato e cifrato.

Nel testo entrava gran parte della cultura classica rinascimentale, da Aristotele a Platone, da Cicerone a Plinio, e Quintiliano, Parmenide, Alberto Magno, Galeno, Diosco-

\footnotetext{
52 Ivi, p. 352.

53 Alessandro Citolini da Serravalle, La Tipocosmia, Venezia, appresso Vincenzo Valgrisi, I 56 I, p. 549.

${ }^{54}$ Lisa Della Giustina, La «Tipocosmia» di Alessandro Citolini (I 56I). Nuove forme di enciclopedismo nel XVI secolo, "Archivio Storico Italiano", CLVII (1999), pp. 63-87.
} 
ride, Teofrasto, Tolomeo, ma anche l'Erasmo degli Adagia, e autori dell'umanesimo riformato come Gioacchino Vadiano. ${ }^{55}$ Utilizzando i molteplici nascondimenti e cautele dei letterati del Cinquecento, inoltre, in alcuni passi molto noti, il nicodemita Citolini faceva abilmente affiorare il proprio dissenso, contrapponendo ad esempio una Chiesa primitiva, immagine trasparente di una Chiesa riformata, in cui «solamente» erano la grazia, i sacramenti, il battesimo, la cena del Signore, la fede, il decalogo, la penitenza, la confessione, la preghiera e soprattutto il Padre Nostro, alla Chiesa dei suoi tempi, la Chiesa di Roma, descritta in un interminabile elenco di pagine e pagine come costruzione esteriore di gerarchie e di riti, di «buone opere» e di libero arbitrio. ${ }^{56}$

Quel che più infiammava, tuttavia, l'eretico' Citolini era la difesa del volgare, di «questa nostra lingua» che

senza altro aiuto d'Imperii, a ciò secondo la opinion d'alcuni sovra ogni altra cosa necessarii, con questo mezzo solo, non pure allargherà maggiormente i già larghissimi termini suoi, ma senza paura che di lei intervenga quello che de l'altre tutte è intervenuto, potrà al paro de 'l Mondo durando tutta intera e salda mantenersi. ${ }^{57}$

Egli assegnava alla lingua italiana una missione internazio-

\footnotetext{
55 L'umanista e medico Gioacchino Vadiano, riformatore svizzero della città di San Gallo, fu autore di un atlante mondiale, l'Epitome trium terrae partium, Asiae, Africae et Europae - uno tra i primi atlanti ad includere l'America - ispirato alla geografia biblica e condannato fin dal I 549 nell'Indice di Venezia (Index des livres interdicts, a cura di Jesús Martinez De Bujanda, vol. III, Index de Venise I 549. Index de Venise et Milan I 554 , Genève, Droz, I987, pp. I69-70). Così veniva citato da Citolini: per sapere «come Adamo poté esserne scacciato fuori, non essendo però scacciato fuor de'l Mondo, leggete il trattato di Giovachin Vadiano, fatto sopra tal proposito", La Tipocosmia, p. 297.

${ }^{56}$ La Tipocosmia, p. I69.

${ }^{57}$ Ivi, p. 552
} 
nale, come già aveva fatto nella Lettera in difesa della lingua volgare, quando si rallegrava che questa lingua avesse

pur già passate l'alpi; e è in Francia da moltissimi conosciuta, amata, e havuta cara [...] Per la Spagna ancora [...] ella è assai ben conosciuta. E [...] nell'isole di Maiolica vi sono di lei le publiche scuole. E odo che nella Magna, e perfin nell'Inghilterra vi si trovan più conoscenti di questa lingua, che di quella. E non passerà molto, ch'ella si stenderà troppo più oltre, che i suoi nemici non pensano. ${ }^{58}$

La diffusione della lingua italiana in Inghilterra è fenomeno ben noto e studiato, soprattutto per quel che riguarda l'età elisabettiana. Assieme allo spagnolo e al francese fu lingua accreditata alla corte di Elisabetta, che già a diciassette anni si esercitava nel volgare italiano, dedicando a re Edoardo, suo fratello, una versione dall'italiano in latino di un sermone dell'esule Bernardino Ochino, acclamato predicatore negli ambienti di corte. ${ }^{59}$ Per Alessandro Citolini il «sermone italico» rappresentò dunque un importante salvacondotto. Nella sua Grammatica de la lingua italiana - dedicata ad un gran dignitario della corte, Cristoforo Hatton, tuttora inedita ma oggi ben nota, «una sorta di manuale per l'insegnamento dell'italiano agli stranieri» - egli presentava con molto orgoglio un particolare metodo di apprendimento e di pronunzia per «la vera, e naturale prolazione di essa lingua, senza andare in Italia ad apprenderla». ${ }^{60}$ Certamente già composta in Italia in seguito agli stretti rapporti d'amicizia con Claudio Tolomei, la grammatica era infatti uno strumento adatto per

\footnotetext{
${ }^{58}$ La lettera d'Alessandro Citolini in difesa della lingua volgare, c. I $5 v$. ${ }^{59}$ Vittorio Gabrieli, Bernardino Ochino: "Sermo de Christo». Un inedito di Elisabetta Tudor, "La Cultura», 2 I (1983), pp. I 5 I-74.

${ }^{60}$ Maurizio Vitale, Senesismi in un grammatico settentrionale del Cinquecento, in Id., Divagazioni linguistiche dal Trecento al Novecento, Firenze, Franco Cesati, 2006, pp. 45-52, con bibliografia.
} 
insegnare a stranieri come lo Hatton - «desideroso de la intera cognizione de la lingua Italiana, sì come si veggono essere tanti altri Principi, e nobili di questo Regno e di tante altre Nazioni ancora ${ }^{61}$ - un metodo di apprendimento e di pronunzia, un metodo ortofonico, ${ }^{62}$ per "gl'innamorati di questa lingua», lingua superiore a molte altre e che si stava diffondendo in tante corti straniere.

\section{La "questione della lingua» fuori d'Italia}

Il mondo degli esuli religionis causa fu dunque ricco di personalità che contribuirono alla diffusione della cultura e della lingua italiana nelle corti europee, soprattutto in Inghilterra, dove la politica di Edoardo vi e dell'arcivescovo Thomas Cranmer favorì l'immigrazione religiosa. Dalla penisola erano già arrivati a Londra Bernardino Ochino, Pier Martire Vermigli ed Emanuele Tremellio, famoso ebraista e traduttore; altri ne giungeranno in anni successivi. ${ }^{63}$ Sebbene l'Inghilterra dovesse apparire loro "un rifugio troppo estraneo e remoto» rispetto alle più vicine mete della Svizzera o della Francia, grazie all'attività dell'ex francescano fiorentino Michelangelo

\footnotetext{
${ }^{61}$ Lina Fessia, Alessandro Citolini esule italiano in Inghilterra con documenti inediti, "Rendiconti» del R. Istituto Lombardo di Scienze e Lettere, cl. Lettere, LXXIII (I939-I940), pp. 2I3-43 (in particolare pp. 235-36); Carmelina Naselli, Alessandro Citolini e la sua inedita grammatica italiana, "Lingua Nostra», IV (I942), pp. 5 I-56.

${ }^{62}$ Vitale, Senesismi in un grammatico settentrionale del Cinquecento, p. 47.

${ }^{63}$ Sul contributo culturale degli italiani alla Riforma europea gli studi sono vasti. Si veda il pionieristico e sempre illuminante saggio di John Tedeschi, The Cultural Contributions of Italian Protestant Reformers in the Late Renaissance, in Libri, idee e sentimenti religiosi nel Cinquecento italiano, Modena-Ferrara, Panini, I987, pp. 8I-I08; più recentemente Michael Wyatt, The Italian Encounter with Tudor England. A Cultural Politics of Translation, Cambridge, Cambridge University Press, 2005; M. Anne Overell, Italian Reform and English Reformation, c. I535-c. I585, Aldershot-Burlington, Ashgate Publishing Company, 2008.
} 
Florio, amico del Citolini e anch'egli esule fin dal I 550, si formò a Londra una comunità di rifugiati italiani religionis causa: la frequentavano molti inglesi, infatuati della moda, dei costumi, dalla civiltà italiana, "per assuefare l'orecchio all'italiano parlato con naturale scioltezza». ${ }^{64}$

L'influenza dei lontani dibattiti sulla lingua riecheggia anche negli scritti del Florio, prima predicatore della «Ecclesia peregrinorun» degli Italiani, poi esautorato e divenuto insegnante di lingua italiana tra la nobiltà di corte, presso la quale aveva cercato protezione e favori. Frutto dei suoi servizi nella casa del duca di Suffolk, Henry Gray, fu un'altra piccola grammatica, le Regole de la lingua thoscana, dedicata alla figlia del duca, Jane, la sfortunata lady, regina d'Inghilterra per soli nove giorni, condannata al patibolo da Maria Tudor. ${ }^{65}$ Testimonianza singolare di una convergenza di interessi linguistici e di protesta religiosa, la grammatica del Florio raccoglieva le regole e «l'instituzioni della nostra lingua thoscana, la quale ragionevolmente per la sua bellezza è da molti stimata, e in gran' pregio da i più belli, e dotti spiriti tenuta»: era ricca, la grammatica, di esempi provocatori: come quello per distinguere tra l'uso del congiuntivo e quello del condizionale: «S'io ubbidisse al papa, ad antichristo ubbidirei»; oppure per illustrare il participio: «quando fui tenuto in pregio dal Papa, non ero amato da Dio. Hora che dal Papa sono perseguitato, son' certo che da Dio sarò tuttavia diffeso»". ${ }^{66}$

Approdato in Valtellina negli anni Sessanta, il Florio intraprendeva la faticosa impresa della traduzione per conto del

\footnotetext{
${ }^{64}$ Luigi Firpo, La chiesa italiana di Londra nel Cinquecento e i suoi rapporti con Ginevra, in Id., Scritti sulla Riforma in Italia, Napoli, Editrice Politecnica, I 996, pp. I 17-94 (in particolare pp. I 22-23).

${ }^{65}$ Giuliano Pellegrini, Michelangelo Florio e le sue "Regole de la lingua thoscana», "Studi di Filologia Italiana», XII (I954), pp. 77-204.

${ }^{66}$ Ivi, pp. I70, I73.
} 
Froben di Basilea del Re metallica di Giorgio Agricola. ${ }^{67}$ Nella prefazione, riprendeva con vivacità le antiche discussioni, e in nome della internazionalizzazione della lingua italiana si schierava ormai apertamente tra le schiere anti-bembiane:

Io non dubito punto, benigno lettore, che alcuni capricciosi, de la lingua Toscana studiosi, non m'habbiano a tacciare in molte cose di questa mia tradozzione. Diranno primieramente che io non habbia osservate a puntino tutte quelle regole del parlare, e de lo scrivere, le quali essi o nel Bembo, o nel Fortunio si trovano haver studiate et apparate. Dipoi che io non mi sia servito, sicome harei potuto fare, di molti vocaboli usati dal Boccaccio, dal Petrarca, e da Dante. Diranno ancora che ad alcuni stromenti nominati in questo libro, io non habbia dato quei nomi apunto che fa la lingua Fiorentina: e forse anche si lasceranno uscir di bocca che in qualche passo questa mia tradozzione sia molto scura, e dico tanto che alcuni non ne potranno con questa agevolezza che vorrebbano, cavar costrutto.

La sua traduzione, tuttavia, non doveva esser letta dai contemporanei dell'età del Boccaccio «ma de la presente»: «i parlari da l'hora in qua si sono mutati, come dal di a la notte». In quanto esule, inoltre, egli aveva la prospettiva di insegnare l'italiano a «molti» che non comprendevano il toscano né avevano mai letto il Boccaccio, né le prose del Bembo:

Quantunque io m'avessi potuto agevolissimamente caminare per le pedate del Bembo, io non l'ho voluto fare, perché questa mia tradozzione non ha esser letta solamente da que' che avranno studiato minutamente le sue prose, ma da molti che eziandio che non l'havranno forse mai sentito nominare: et oltre a ciò che quando il leggessero, non l'intenderiano che tanto, o quanto, per non esser Toscani, né aver studiato le Cento novelle. ${ }^{68}$

\footnotetext{
${ }^{67}$ Firpo, Giorgio Agricola e Michelangelo Florio, in Id., Scritti sulla Riforma in Italia, pp. 245-59.

${ }^{68}$ Giorgio Agricola, L'arte de' metalli. Tradotto in lingua toscana da Michelangelo Florio, fiorentino, prefazione di Luigi Firpo, Torino, Bottega
} 
Questa traduzione, condotta quando il Florio era riottoso ministro riformato della piccola comunità di Soglio, in Val Bregaglia, riecheggiava le discussioni linguistiche conosciute in patria, ma rispondeva anche a esigenze diffuse tra molti degli esuli in quei decenni. Del longevo pastore di Chiavenna, Scipione Lentolo, ad esempio, era l'opera Italicae grammatices Praecepta et Ratio, edita a Ginevra nel I 567, scritta anche in questo caso, a quanto sembra, per insegnare la lingua italiana agli stranieri. ${ }^{69}$

Per la questione linguistica, tuttavia, la polemica antibembiana e la dichiarata intenzione di abbandonare "ogni affettazione de' fastidiosi Toscanismi», in nome dell'uso di parole ed espressioni piane e naturali, "per darla bene ad intendere a le persone semplici», venivano soprattutto dagli italiani esuli a Ginevra, impegnati in traduzioni in volgare, sia per la comunità italiana, sia per il mercato clandestino nella stessa Italia. ${ }^{70} \mathrm{Si}$ tratta di una "questione della lingua» che, in maniera affascinante, si era trasferita anche fuori d'Italia, tra gli esuli, a volte polemici nei confronti dei «toscanismi» e della "volgar lingua boccacesca» perché, come scriveva ancora il Florio nella sua traduzione dell'Agricola, «se a gli stromenti nominati in questo libro io havessi dato solamente $\mathrm{i}$ nomi usati a Firenze, gl'honorati Frobenii per li quali l'ho tradotto, si sarebbero potuti giustissimamente dolere di me, con dirmi

d'Erasmo, I969, «Lettera di Michelangelo Florio fiorentino al benigno Lettore».

${ }^{69}$ Tedeschi, The Cultural Contributions of Italian Protestant Reformers, $\mathrm{p}$. I05. Sul Lentolo e la sua grammatica cfr. Emanuele Fiume, Scipione Lentolo I 525-I 599. "Quotidie laborans evangelii causa", Torino, Claudiana, 2003, p. I I I.

70 Sulla presenza «di una riflessione locale sul volgare da utilizzare, di un'indigena "questione della lingua" nella Ginevra calvinista e italiana", cfr. Franco Pierno, Una retrodatazione di "Toscanismo» e appunti su una "questione della lingua» nella Ginevra di Calvino, "Lingua Nostra», LXv (2004), n. I-2, pp. 6-I 5, in particolare p. 7. 
che essi non me l'hanno fatto tradurre per venderlo solamente a Firenze, ma in ogni altra parte d'Italia». ${ }^{71}$

La traduzione del Florio fu anche "probabile frutto di contatti ${ }^{72}$ con un altro famoso esule in Valtellina, Lodovico Castelvetro, anch'egli in quegli anni nella vicina Chiavenna, perché già condannato in contumacia per eresia dall'Inquisizione nel novembre del I 560 , con accuse ancora oggi in parte oscure cui, com'è noto, aveva fatto da detonatore anche la famosa querelle con Annibal Caro.

\section{Lodovico Castelvetro}

Sul modenese Lodovico Castelvetro gli studi sono ormai vastissimi, dovuti all' «interesse crescente, quasi l'entusiasmo»" ${ }^{73}$ da parte degli studiosi, a partire dall'importante saggio di Ezio Raimondi, Gli scrupoli di un filologo. Lodovico Castelvetro e il Petrarca. ${ }^{74}$ L' «austero polemista - a detta del Raimondi - eversore di tutte le autorità e di tutti i giudizi tradizionali», «filologo razionalista» dalla "spigolosa intelligenza», uomo dal pessimo carattere ma con un orecchio e una memoria sensibilissimi, fu «un filologo riformato, un ribelle alle forme tradizionali, e tuttavia devoto, anche in esilio, a una grande letteratura». ${ }^{75}$ Per Castelvetro si è dunque parlato anche di

\footnotetext{
${ }^{71}$ Agricola, L'arte de’ metalli, «Lettera di Michelangelo Florio fiorentino al benigno Lettore», citato anche in J. Tedeschi, The Cultural Contributions of Italian Protestant Reformers, p. 99.

${ }^{72}$ Firpo, Giorgio Agricola e Michelangelo Florio, p. 259. Per Frances Amelia Yates, John Florio. The Life of an Italian in Shakespeare's England, Cambridge, Cambridge University Press, 20 Io (I ed. I934), p. 23, gli attacchi al Bembo che si ritrovano nel De Metallurgia sarebbero stati ispirati proprio dal Castelvetro e dalla sua polemica con il Bembo, che nel I 563 aveva fatto molto rumore.

${ }^{73}$ Marazzini, Castelvetro linguista, p. I90.

${ }^{74}$ In Rinascimento inquieto, Torino, Einaudi, I994, pp. 57-I 42.

${ }^{75}$ Ivi, p. I05.
} 
«eresia critica», speculare alla probabile eresia religiosa, accanita "contro ogni retorica del vago e dell'oscuro, ogni deposito mitologico e dottrinario affidato ad interpreti sacerdotali». ${ }^{76}$

Castelvetro, com'è noto, fu animatore a Modena, negli anni Quaranta, di un gruppo di letterati che si riunivano in modo informale, ma aperti alla società modenese e fortemente coinvolti nel dissenso religioso; all'interno di una Accademia, che nel I 545 fu sciolta dal duca d'Este proprio per accuse di eterodossia. I 'ragionamenti' che vi si svolgevano erano 'conversazioni civili' in un contesto municipale, di fatto contrapposti a quelli che avevano come scenario le corti. ${ }^{77}$ Sovente, dunque, la vis polemica del critico modenese, impegnato per anni nelle cariche cittadine e orgogliosamente convinto dell'indipendenza dei patriziati urbani, si volse contro le forme della letteratura posta al servizio dei potenti, «fuggendo egli - scrisse il nipote Lodovico - di servire re e principi grandi solamente per haver la libertà e tempo di poter studiare». ${ }^{78}$

${ }^{76}$ Giancarlo Mazzacurati, Aristotele a corte: il piacere e le regole (Castelvetro e l'edonismo) in Culture et société en Italie du Moyen-Âge à la Renaissance. Hommage à André Rochon, Paris, Université de la Sorbonne Nouvelle, I985, pp. 265-83 (in particolare pp. 270-7I). Per Mazzacurati, il «grammaticuccio» modenese era un lettore «luterano» anche dei classici, e parlava di un'arte poetica che doveva essere visibile e leggibile, non allusiva a sapienze arcane, allegoriche o simboliche, dedita alla ricerca di chiarezza e semplicità; il lettore doveva essere tutto rivolto al testo, alla cui comprensione bisognava dedicare ogni sforzo: «Ma lo 'ntendere i Poeti non consiste se non nel senso comune, e popolare».

77 Alberto Roncaccia, Il metodo critico di Ludovico Castelvetro, Roma, Bulzoni, 2006, pp. 33-8I. Sull'Accademia modenese lo studio più recente è quello di Lucia Felici, Frammenti di un dialogo. Ludovico Castelvetro e $i$ suoi rapporti con gli accademici modenesi, in Ludovico Castelvetro. Letterati e grammatici, pp. 3 I 5-55.

${ }^{78}$ Citato in Davide Dalmas, Dante nella crisi religiosa del Cinquecento italiano. Da Trifon Gabriele a Lodovico Castelvetro, Manziana (Roma), Vecchiarelli, 2005, p. I87. 
Convinzione che ne determinò tutta la vita, e che lo portò a schernire anche l'amico carissimo Filippo Valentini, essendo questi entrato nella famiglia del cardinal Contarini: "voi sete servitore altrui, né di questa servitù uscirete forse mai». ${ }^{79}$

Sua è una bella pagina 'democratica', nella Poetica di Aristotele vulgarizzata e sposta, sulla commedia e sulla tragedia antiche, che parlavano liberamente contro «esso tiranno o i re o i suoi cortigiani o i pochi»; e sulla commedia "nuova», che, invece, era "carissima allo stato de' tiranni, de' re e de' pochi», perché non rimproverava loro nulla, né li minacciava di punizione divina, né incitava il popolo «a conservare la libertà e a portare odio a' tiranni, sì come a persone ingiuste e dispiacenti a Dio»:

La commedia antica, che nominatamente metteva in favola le persone conosciute, non può aver avuto luogo sotto lo stato de' tiranni o de' re o de' pochi, percioché o esso tiranno o i re o i suoi cortigiani o i pochi, sì come conosciuti, e per la possanza prendendosi ogni licenza di fare e di dire contra le leggi e 'l dovero, sarebbero soggetto e segno nel quale ferirebbe tuttavia l'arco della commedia; sì come altresì la tragedia non sarà mai graziosa sotto questo stato, rimproverandosi a' tiranni o a' re o a’ pochi con l'essempio altrui le loro operazioni e minacciandosi loro la debita pena per giusta sentenzia divina. Ma la comedia nuova è carissima allo stato de' tiranni, de' re e de' pochi, percioché non rimprovera loro niuna loro operazione, né minaccia loro punizione niuna, né solleva il minuto popolo, né il commuove a passione niuna, essendo l'azioni rappresentate di dispiacere non grande e mitigato da sopravegnente alegrezza. Sì come d'altra parte la tragedia è di grandissimo utile e molto piace allo stato popolare, confermandosi il popolo a conservare la libertà e a portare odio a' tiranni, sì come a persone ingiuste e dispiacenti a Dio, li quali sotto l'esempio altrui sono proposti dinanzi a gli occhi del popolo. ${ }^{80}$

\footnotetext{
${ }^{79}$ Roncaccia, Il metodo critico di Ludovico Castelvetro, p. 85.

${ }^{80}$ Lodovico Castelvetro, Poetica d'Aristotele vulgarizzata e sposta, a cura di Werther Romani, Roma-Bari, Laterza, I978, p. 6I. Questa pagina è citata
} 
Come il Citolini, anche il Castelvetro, scriverà il Muratori, ${ }^{81} \mathrm{fu}$ «perdutamente innamorato» della lingua italiana e partecipò attivamente all'intensa discussione filologica e grammaticale del suo secolo, che avvenne a colpi di trattati, di commenti, di lettere private: negli anni Cinquanta Castelvetro sosteneva, ironicamente, di avere accese «cento liti grammaticali» in mezza Italia, da Venezia, a Bologna, a Firenze, e con molteplici avversari. ${ }^{82}$ Il suo metodo dissacratorio non riconosceva né culture accademiche dominanti, né principio di autorità, fosse questa letteraria, politica o religiosa; criticava persino il «gran Bembo», da cui pur dipendeva fortemente per la sua

dal Raimondi come "pagina che avrebbe entusiasmato, si può esserne certi, persino l'Alfieri», Gli scrupoli di un filologo, p. I42.

${ }^{81}$ Lodovico Castelvetro, Opere varie critiche, non più stampate colla vita dell'autore, a cura di Lodovico Antonio Muratori, Berna, Pietro Foppens, I727, p. 68.

${ }^{82}$ Enrico Garavelli, "Nelle tenzoni alcuna volta si commenda una sottigliezza falsa più che una verità conosciuta da tutti», in Omaggio a Lodovico Castelvetro (I 505-I 57I), Atti del seminario di Helsinki, I 4 ottobre 2005, a cura di E. Garavelli, Helsinki, Publications du Département des Langues Romanes de l'Université de Helsinki, I6 (2006), pp. 86-87. Era un dibattito che stava a cuore anche ad altri letterati modenesi a lui vicini, sostenitori della supremazia del volgare sul latino. In una ben nota disputa sulla questione della lingua, i modenesi Paolo Sadoleto e Antonio Fiordibello, appoggiati dal cardinale Jacopo Sadoleto, avevano sostenuto che «la lingua vulgare non era da adoperare se non in inscrivere cose famigliari, et si doveva scrivere senza riguardo, et nella guisa che di tempo in tempo si parla tra corteggiani di Roma, e le cose gravi, et degne di memoria, si dovevano scrivere nella latina, et nella latina del secolo di Cicerone». Gli accademici Giovanni Fallopia, Alessandro Melano, Francesco Camorana e lo stesso Castelvetro diedero allora incarico a Filippo Valentini di elaborare una risposta, «il quale fece un libro in pochi dì assai lungo et rispose in modo, che essi non scrissero più, né parlarono di questa lite mossa temerariamente» (Ludovico Castelvetro, Racconto delle vite d'alcuni letterati del suo tempo, citato in Filippo Valentini, Il principe fanciullo. Trattato inedito dedicato a Renata ed Ercole II d'Este, a cura di Lucia Felici, Firenze, Olschki, 2000, Introduzione, pp. 26-27). 
iniziale formazione, in nome della libertà di critica, ${ }^{83}$ tanto che Giovan Battista Marino, collocandolo nella sua Galleria, non si peritava di ironizzare chiamandolo «autore inemendabile e perfetto", che lo stesso Iddio non avrebbe voluto presente durante la creazione, nel timore che trovasse «nel Poema del mondo alcun difetto». ${ }^{84}$ La più recente storiografia gli riconosce soprattutto una originalità e una autonomia particolare nella questione della lingua: il suo contributo, ad esempio, alla ricostruzione delle origini dell'italiano e del passaggio dal latino al volgare è, secondo il Marazzini, tra le sue pagine più belle, in una prospettiva "nuova e di eccezionale interesse». ${ }^{85}$

Negli scritti del modenese, prodotti da «uno degli scrittoi più interessanti del secondo Cinquecento ${ }^{86}-$ con il metodo frammentario che gli era proprio, per appunti, per "quadernucci» ${ }^{87}-$ anche Castelvetro, come Citolini, introdusse pensieri e osservazioni che, in modo nicodemitico, alludevano alle proprie propensioni religiose, alla difficoltà di condividere convinzioni pericolose, alla facilità con cui si potevano ricevere accuse d'eresia. Gli atti di un recente convegno romano a lui dedicato si intitolano Filologia e ascesi, proprio per alludere al nesso tra rigore umanistico e sensibilità religiosa, con un richiamo a quel suo ruolo «straordinariamente atipico sul piano filologico e spirituale», e alla sua "filologia come ascesi», cioè come «esercizio strenuo, instancabile escavazione

\footnotetext{
${ }^{83}$ Roncaccia, Il metodo critico di Ludovico Castelvetro, p. I 36

${ }^{84}$ Citato in Stefano Jossa, Filosofi e letterati. Muratori e Fontanini interpreti della contesa tra Castelvetro e Caro, in Ludovico Castelvetro. Letterati e grammatici, p. I 28.

${ }^{85}$ Marazzini, Castelvetro linguista, p. 196.

${ }^{86}$ Matteo Motolese, Le carte di Lodovico Castelvetro, "L'Ellisse. Studi Storici di Letteratura Italiana", I (2006), pp. I63-9I, in particolare p. I63.

${ }^{87}$ Matteo Motolese, Il codice $\alpha$ S. 5 . I della Biblioteca Estense di Modena tra diacronia e sincronia. Alcuni appunti, in Lodovico Castelvetro, Filologia e ascesi, a cura di Roberto Gigliucci, Roma, Bulzoni, 2007, p. 48.
} 
semantica e servizio eroico della verità, ma anche scarnificazione lucida e razionale dell'oggetto senza preoccupazione di alcun dogmatismo o idea ricevuta». ${ }^{88}$

Petrarca veniva censurato nel suo "stile pagano» attraverso le Lettere di san Paolo; «l'ombra di Erasmo» si proiettava a volte sulle pagine; si insinuavano accenti e pensieri che richiamavano in modo coperto il pensiero dei riformatori, sulla grazia o sul libero arbitrio; e persino, in un passo molto elaborato, il pensiero di Calvino, quando Castelvetro discuteva sul bene e sul male "a gloria di Dio» e per "castigare gli eletti suoi». ${ }^{89}$ Nelle Chiose all'Eutifrone di Platone, in modo come sempre oscuro e allusivo, il letterato modenese introduceva anche una critica all'Inquisizione dei suoi tempi, quando sosteneva che nelle accuse di «rea credenza», e quindi di eresia, tra i pagani si «nominava l'accusatore, non che i testimoni [...] là dove appresso noi altri si celano gli accusatori, e i testimoni». Il controllo della «rea credenza» tra i pagani, per di più, era affidato alla politica, "al reggimento comunale», alla città che "come madre mai non si condurrebbe ad uccidere i figliuoli, quantunque dessero noia a gli altri fratelli», e non, come accadeva con i tribunali inquisitoriali, a persone esterne «che non fossero della cittadinanza». ${ }^{90}$ Osservazioni che sono disseminate ovunque, ma senza prendere partito, mantenendo la propria indipendenza, giocando a volte sull'ironia, come quando nella Sposizione a XXIX Canti dell'Inferno dantesco, composta negli anni dell'esilio, proponeva di porre accanto al purgatorio anche un 'premiatorio' «nel quale godessero per alcun tempo le anime dannate per gli beni fatti, che non sono a vita eterna». ${ }^{91}$

${ }^{88}$ Roberto Gigliucci, Premessa, in Ludovico Castelvetro. Filologia e ascesi, pp. 9-Io.

${ }^{89}$ Raimondi, Gli scrupoli di un filologo, pp. I04-07.

${ }^{90}$ Castelvetro, Opere varie critiche, p. 284.

${ }^{91}$ Dalmas, Dante nella crisi religiosa del Cinquecento italiano, p. 206. 
Testimone della vicinanza di Castelvetro alle idee riformate che circolavano negli anni Quaranta, com'è noto, è soprattutto la sua volgarizzazione clandestina di uno scritto di Filippo Melantone, Dell'autorità della Chiesa et degli scritti antichi, condotta sotto falso nome, fedelissima al testo anche nei suoi meccanismi retorici..$^{92} \mathrm{Il}$ «libricciuolo», tradotto probabilmente nel I54I per gli amici modenesi, e soprattutto per coloro che non sapevano le lingue, ${ }^{93}$ testimonia di un'adesione alla polemica protestante contro "la tirannaria» dei pontefici e contro il progressivo pervertimento della dottrina del Vangelo dai tempi della chiesa primitiva, contro le false "cerimonie» e l'idolatria insita nel culto dei santi, «la presente adoratione degl'idoli».94 Il metodo critico del Castelvetro, umanistico e razionalista, era influenzato dal Valla, ma anche da esponenti dell'umanesimo d'oltralpe, dall'Agricola al Melantone, dal Butzer al Pellikan; ${ }^{95}$ una compagine culturale umanistica europea, quindi, che aveva promosso un ampio dibattito antiscolastico sul rinnovamento della dialettica e per un linguaggio,

\footnotetext{
${ }^{92}$ Lorenzo Geri, Castelvetro traduttore, in Lodovico Castelvetro. Filologia e ascesi, pp. 24I-59.

${ }^{93}$ Edito in Ludovico Castelvetro. Filologia ed eresia. Scritti religiosi, a cura di Guido Mongini, Brescia, Morcelliana, 20 I I, pp. 173-249, in particolare p. 227.

${ }^{94}$ Interessanti sono anche le "Chiose de' vocaboli greci et d'alcune altre cose», edite dal Mongini (Filologia ed eresia, pp. 227-30), dove, ad esempio, l'etimologia della parola "messa" mostra le posizioni riformate del Castelvetro nei confronti dell'eucarestia e suona così: "Messa in lingua soriana, là dove furono le Chiese primiere, significa tributo, et colta. Et certo nella cena del signore si paga la gabella sacra, cioè il ringratiamento, le lodi, le limosine per gli poveri et la dimostratione di gratitudine per la salute ricuperata. Laonde anchora Hesichio interprete del Levitico dice di niuna altra cosa essere oblatione nella cena del signore che di gratie» (pp. 228-29).

95 Claudia Rossignoli, "Dar materia di ragionamento». Strategie interpretative della "Sposizione", in Lodovico Castelvetro. Filologia e ascesi, p. Ioo.
} 
come "paradigma della conoscenza della realtà», che potesse affrontare anche le scienze più ardue, dalla teologia, alla medicina, alla giurisprudenza, e che aveva applicato questa riforma linguistica ed epistemologica anche alle Sacre Scritture. ${ }^{96}$

La recente acquisizione da parte di Andrea Barbieri di un libro di conti del Castelvetro, della fine degli anni Quaranta, testimonia dello straordinario patrimonio librario del letterato modenese, messo a disposizione della collettività cittadina attraverso prestiti di libri a stampa e manoscritti della sua biblioteca: circa un centinaio di libri prestati e restituiti in otto anni, tra i quali si ritrovavano testi classici latini e greci, autori moderni come Valla o Poliziano, e anche, con «una eccezionale apertura di prospettiva sull'umanesimo d'oltralpe» soprattutto riformato, commentari biblici o traduzioni del Münster, di Erasmo, di Martin Bucero, di Filippo Melantone, oltre a Guillaume Budé, Rodolfo Agricola, Thomas Linacre, Lazare de Baï. «Si ha l'impressione, per l'ultima volta prima degli esiti del Concilio di Trento, di una comunità europea del sapere», ${ }^{97}$ appartenente a una internazionalità intellettuale, a una «repubblica di dotti», di letterati e grammatici, che l'azione dell'Inquisizione in Italia e dell'Indice dei libri proibiti scompaginò, venendo a incidere almeno apparentemente su una libera circolazione di uomini, di libri e di cultura. Castelvetro come Citolini, dunque, già prima della fuga e dell'esilio fecero saldamente parte in Italia di «una rete di rapporti (ancora in molta parte da indagare) tra intellettuali che si scambiano libri, discutono per lettera e a voce [...] si arrovellano sulle medesime problematiche grammaticali». ${ }^{98}$

\footnotetext{
${ }^{96}$ Ivi, pp. I I-02. Erika Rummel, The Humanist-Scholastic Debate in the Renaissance and Reformation, Cambridge, Harvard University Press, I995. ${ }^{97}$ Andrea Barbieri, Castelvetro, i suoi libri e l'ambiente culturale modenese del suo tempo, in Lodovico Castelvetro. Filologia e ascesi, p. 62.

${ }^{98}$ Matteo Motolese, Introduzione a: Ludovico Castelvetro, Giunta fatta
} 
Già in fuga nel 1560 , ma ancora nascosto in Italia, nella primavera del I 56I, il letterato modenese era costretto all'esilio, prima a Chiavenna, poi a Ginevra, infine a Lione. Nel I 569 era a Vienna, alla corte tollerante e cosmopolita di Massimiliano II d'Asburgo, ${ }^{99}$ al quale dedicherà la sua traduzione della Poetica di Aristotele. Era una corte dove «tutti parlavano l'italiano", ${ }^{100}$ dove la cultura italiana, dalle lettere alle arti, era tenuta in gran conto, come pure il pensiero umanistico e l'irenismo di ascendenze erasmiane. L'esule Castelvetro, dunque, si rivolgeva all'imperatore offrendogli «l'umile e 'l picciolo dono" della Poetica "volgarizzata e sposta da me», per ringraziare il sovrano che lo aveva accolto, insieme al fratello, entrambi travolti «da fiero e fortunoso temporale», in un «tranquillo e sicuro porto». Lodava l'imperatore perché «è veramente meravigliosa quella piena conoscenza e notizia che ha delle diverse e molte lingue, e spezialmente dell'idioma italiano, col quale favella così puramente e così vagamente che assai chiaro appare che non v'habbia posto meno studio che in alcuno degli altri».

Pensava, il Castelvetro, che quella sua fatica sarebbe stata gradita al sovrano perché scritta in una lingua, l'italiano, «nella quale non niego d'avere speso qualche tempo per impararla e per avanzarmi alquanto in essa». Era una traduzione che rifletteva le sue convinzioni più profonde sulla lingua, a dimostrare che era possibile che «con le voci proprie e naturali di questa lingua» si potessero esprimere

al ragionamento degli articoli et dé verbi di Messer Pietro Bembo, p. XIv. ${ }^{99}$ Howard Louthan, The quest for compromise. Peacemakers in CounterReformation Vienna, Cambridge, Cambridge University Press, I 997.

${ }^{100}$ Dirk J. Jansen, Gli strumenti del mecenatismo: Jacopo Strada alla corte di Massimiliano II, in «Familia» del Principe e famiglia aristocratica, a cura di Cesare Mozzarelli, Roma, Bulzoni, I988, pp. 7 I I-43. 
altri concetti della mente nostra che d'amore e di cose leggiere e popolari, e si potesse ragionare e trattar d'arti e di dottrine e di cose gravi e nobili, senza bruttare e contaminar la purità sua con la 'mmondizia delle voci barbere e scolastiche, e senza variare e alterar la semplicità sua con la mistura delle voci greche e latine, quando la necessità non ci costringe a far ciò, acciocché riconoscendosi la sufficienza e 'l valore di questa lingua ancora in questa parte, non resti priva più lungamente della debita sua lode. ${ }^{101}$

La consonanza di alcune posizioni del Castelvetro con quelle del Citolini, non solo dal punto di vista religioso, ma anche linguistico, mostra dunque in certo qual modo un percorso comune. L'acquisita convinzione che la «nostra lingua commune», l'«idioma italico», fosse ormai maturo per trattare ogni materia, per allargarsi a ogni disciplina e per conquistare anche l'Europa, sembra condivisa da questi esuli italiani che, separati dalla patria per le loro posizioni religiose, credevano profondamente che loro rimanesse il compito di propagandare in Europa il «valore» della lingua italiana.

${ }^{101}$ Castelvetro, Poetica d'Aristotele vulgarizzata, p. 4. 

PARTe SECONDA 



\title{
Silvia Morgana
}

\author{
«HO FATO TRANSLATARE IN LINGUA NOSTRA» \\ Tra Milano e Ticino ducale: \\ LINGUA CANCELLERESCA E VARIETÀ D'USO
}

Giuseppe Parini nelle sue lezioni di Belle Lettere (I770) ${ }^{1}$ tracciava ai suoi discepoli di Brera un ampio profilo di storia della lingua italiana, dal Duecento fino ai suoi tempi (una settantina di carte nell'autografo ambrosiano), ${ }^{2}$ e delineava la prima espansione della lingua toscana grazie alle Tre Corone del Trecento fiorentino: $\mathrm{i}$ «tre sublimi spiriti» che «in pochissimo tempo sì grandi ali le diedero, che fuori la spinsero dal suo nido e la fecer volare per tutta l'Italia con felicissimi augurj, e costor furono Dante Alighieri, Francesco Petrarca, e Gio[vanni] Boccaccio». ${ }^{3}$

Ho sempre trovato molto suggestiva questa immagine, con cui Parini intendeva spiegare ai suoi studenti «per qual ragione la favella, che ora è propria dei Toscani ebbe poscia tale predominio sopra tutti i dialetti delle altre Provincie», tanto che "sola poi divenisse la lingua nobile comune a tutta l'Italia». ${ }^{4} \mathrm{Mi}$ fa particolarmente piacere evocare in questa sede l'illustre professore «Milanese di Bosisio»:5 perché qui, tra le

\footnotetext{
${ }^{1}$ La cattedra di Eloquenza e Belle Lettere fu ricoperta da Parini dal I 770 sino alla fine del I798, cfr. Giuseppe Parini, Prose I. Lezioni. Elementi di retorica, a cura di Silvia Morgana e Paolo Bartesaghi, Milano, Led, 2003.

${ }^{2}$ Che cosa sia l'Arte del Dire. Articolo 4.to dell'origine, de' progressi, della natura, dell'uso e del presente sistema della Lingua Italiana, in Parini, Prose I. Lezioni, cit., pp. I98-25I.

3 Ivi, p. 206.

${ }^{4}$ Ivi, p. 202.

${ }^{5}$ Così lo definiva in tono spregiativo, per le origini brianzole, il Padre Onofrio Branda, suo avversario nel corso della celebre polemica del i76o.
} 
mura di Brera, ha passato molti anni insegnando l'eloquenza e il significato civile delle Belle Lettere a più di una generazione di giovani milanesi nell'epoca delle grandi riforme austriache. E poi anche perché è diventato, da un po' di tempo in qua, uno degli amici più cari del nostro Maestro, che oggi festeggiamo, perché l'accompagna quotidianamente nelle sue giornate di studio. I frutti di questo sodalizio li vedremo tra non molto. ${ }^{6}$

Dunque Parini storico della lingua italiana, riguardo alla funzione avuta dai «tre illustri scrittori» nel processo di italianizzazione della penisola, arrivava a concludere che «l'Italia dee spezialmente riconoscer da essi lo stabilimento e la perfezione della Toscana lingua, e dalle loro opere la promulgazione di essa talmente che poi è divenuta comune a tutti gl'Italiani, $e$ da ciò ha il nome più generale acquistato d'Italiana». ${ }^{7}$ Nella ricostruzione pariniana del processo di italianizzazione si può trovare una conferma ulteriore che l'italiano in quanto lingua di cultura fu percepito in epoca preunitaria «dalle Alpi alla Sicilia» come l'«elemento primario della identità nazionale», come fortissimo collante identitario, «nonostante la divisione e il frazionamento politico». ${ }^{8}$

Milano documenta esemplarmente questo processo: la sua storia linguistica e culturale si intreccia infatti con la storia

Cfr. G. Parini, Prose. Scritti polemici. Edizione nazionale delle opere di Giuseppe Parini, a cura di S. Morgana e P. Bartesaghi, Pisa-Roma, Fabrizio Serra editore, 20 I 2, p. 2 I I.

${ }^{6} \mathrm{Nel}$ volume in corso di stampa: Maurizio Vitale, La «dizione» formale dell' "Italo Cigno". Notazioni di stile e di lingua nella poesia e nella prosa di Giuseppe Parini.

${ }^{7}$ Parini, Prose I. Lezioni, p. 2 I 3 , miei i corsivi.

${ }^{8}$ Cfr. M. Vitale, La lingua italiana e l'unità nazionale, "Rivista di Storia della Filosofia», 4 (2012), p. 827. Su questi temi cfr. anche Francesco Bruni, Italia. Vita e avventura di un'idea, Bologna, Il Mulino, 2o Io; Lorenzo Tomasin, Italiano. Storia di una parola, Roma, Carocci, 20 I I. 
della progressiva espansione del toscano divenuto lingua italiana comune e poi, a partire dall'epoca illuministica, con il rinnovamento dell'italiano in direzione moderna ed europea, di cui si fa motore trainante. ${ }^{9}$ Devo cedere a questo punto ancora la parola al Maestro, che illustrando la prima diffusione dei modelli letterari e linguistici toscani nella Milano del secondo Trecento, ha sottolineato il ruolo fondamentale della presenza di Petrarca alla corte viscontea (I353-I36I) e il diverso quadro culturale tra la prima e la seconda metà del secolo:

L'influenza del tosco-fiorentino, limitata agli inizi del Trecento, ebbe peso dapprima sulla lingua della poesia più culta per il magistero toscano promosso a Milano dapprincipio da Fazio degli Uberti e testimoniato dall'attività di Brizio Visconti ed ebbe peso di poi per l'autorità del Petrarca nel suo lungo soggiorno milanese. Ma di decennio in decennio, specie nella seconda metà del secolo, con il concorso di poeti tosco-fiorentini e di rimatori veneti dei toscani primi fedeli imitatori, l'influenza toscana divenne sempre più forte e decisiva, consentendo altresì, nel momento della sua maggiore efficacia, l'eliminazione, in ossequio alle forme fiorentine, dei più crudi tratti dialettali anche nelle esperienze linguistiche popolari e locali. ${ }^{10}$

Se spostiamo lo sguardo dall'àmbito letterario a quello dei testi pratici, in un quadro per Milano molto avaro di testimonianze, già a fine Trecento si possono trovare tracce di una precoce infiltrazione del toscano nelle lettere di mercanti milanesi inviate alla compagnia di Francesco Datini di Prato, potente mercante di stoffe. ${ }^{11} \mathrm{Ma}$ è nel corso del Quattrocento

\footnotetext{
${ }^{9}$ S. Morgana, Storia linguistica di Milano, Roma, Carocci, 20 I 2.

${ }^{10}$ Cfr. M. Vitale, Cultura e lingua a Milano nel Trecento, in Petrarca e la Lombardia, a cura di Giuseppe Frasso, Giuseppe Velli, M. Vitale, RomaPadova, Editrice Antenore, 2005, pp. 3 I-49, a p. 49.

${ }^{11}$ Cfr. Morgana, Storia linguistica di Milano, cit., pp. 30-32; L. Frangioni,
} 
che la storia linguistica di Milano diventa progressivamente sempre meno municipale e sempre più italiana, per la convergenza di più fattori: in particolare, le esigenze comunicative e di scambio proprie della cancelleria ducale, che favoriscono, accanto al latino, l'uso del volgare, attestato dal I426, e gli orientamenti culturali e linguistici filotoscani alla corte sforzesca, che culminano nell'età di Ludovico il Moro. Vale la pena sentire ancora la voce di Parini che, con notevole sensibilità storiografica, indicava la politica culturale degli Sforza come determinante per il «risorgimento» del volgare:

Precipua cagione di un tale risorgimento [del volgare] fu il bongusto di Lorenzo de' Medici, autorevolissimo Cittadino Fiorentino, e la dichiarata protezione, ch'egli concedette a' Letterati per cui meritò il cognome di Padre delle Lettere. Coll'esempio e coll'autorità di costui cominciò in Firenze a ritornar nell'antico splendore la Toscana Poesia. Benchénon molto minor merito di Lorenzo ebbero in ciò Gio. Galeazzo Maria Sforza, e Ludovico il Moro zio di lui, amendue duchi di Milano, alla Corte de' quali tutti gli scienziati, e massimamente i Poeti italiani erano bene accolti e protetti. ${ }^{12}$

Grazie soprattutto agli studi di Maurizio Vitale possiamo seguire l'evoluzione nel corso del Quattrocento della koinè

Milano fine Trecento. Il carteggio milanese dell'Archivio Datini di Prato, Firenze, Opus libri, I994. Josh Brown nella sua tesi pho ha identificato e trascritto una ottantina di lettere di scriventi milanesi, inviate da Milano tra il 1380 e il I407, che documentano un forte ibridismo linguistico nella pratica della scrittura, ma anche l'inequivocabile presenza di tratti toscani (addirittura prevalenti, come nel caso dei condizionali, tipo farebe, vorebe) o di ipercorrettismi (quale la sovraestensione del dittongo uo), spia del tentativo di demunicipalizzare la scripta locale adeguandosi al modello linguistico dei corrispondenti (J. Brown, Early evidence for Tuscanisation in the Letters of Milanese Merchants in the Datini Archive, Prato I396-I402, voll. 2, phD Thesis, School of Humanities-Italian Studies, University of Western Australia, 20 I I).

12 Parini, Prose I. Lezioni, p. 22 I; miei i corsivi. 
cancelleresca milanese e della sua variabilità nelle scritture pratiche che la prendono a modello: ${ }^{13}$ una lingua composita, segnata da una patina latineggiante, sempre meno connotata localmente e sempre più contrassegnata da forme fiorentineggianti, che non vanno però considerate come «il semplice risultato [...] di indiretto conguagliamento italiano dovuto agli stretti commerci diplomatici e culturali sovraregionali», ma come il riflesso del grande prestigio della lingua e della letteratura tosco-fiorentina antica e moderna nella corte sforzesca. ${ }^{14}$ La scrittura cancelleresca milanese di fine Quattrocento, conclude Vitale alla fine della sua decisiva indagine,

anziché rivelarsi, nel suo assetto per quanto possibile conguagliato ed eletto, come il corrispettivo linguistico del sogno ludoviciano di potenza di un grande stato milanese, denuncia il suo lento anche se non sempre lineare processo di conformità alla lingua proposta dalla cultura di corte e rappresenta perciò uno dei primi precoci esempi, sul piano della scrittura pratica e prosastica, di capitolazione di una koinè locale di fronte al tipo linguistico tosco-fiorentino, il che equivale a dire al tipo linguistico italiano. ${ }^{15}$

\footnotetext{
${ }^{13} \mathrm{M}$. Vitale, La lingua volgare della cancelleria visconteo-sforzesca nel Quattrocento, Milano, Cisalpino, I953; Id., La lingua volgare della cancelleria sforzesca nell'età di Ludovico il Moro, in Id., La veneranda favella, Napoli, Morano, I988, pp. I69-242; Paolo Bongrani, Lingua e letteratura a Milano nell'età sforzesca. Una raccolta di studi, Parma, Istituto di Filologia moderna dell'Università, I986; Nicoletta Maraschio, Lingua, società e corte di una signoria padana fra Quattro e Cinquecento, in Ludovico Ariosto: lingua, stile e tradizione, a cura di Cesare Segre, Milano, Feltrinelli, I976, pp. 29-38; S. Morgana, Lingue e varietà di lingua nella Milano sforzesca, in Politica, cultura e lingua nella Milano sforzesca, Milano, Istituto Lombardo Accademia di Scienze e lettere, I995, pp. 7-29; Ead., Materiali per la storia della lingua non letteraria. Gride e documenti dell'ultima età sforzesca, in Studi di lingua e letteratura lombarda offerti a Maurizio Vitale, Pisa, Giardini, I983, voll. 2, vol. I, pp. 317-61.

${ }^{14}$ Vitale, La lingua volgare della cancelleria sforzesca nell'età di Ludovico il Moro, cit., pp. 2 IO-I I.

${ }^{15}$ Ivi, p. 226.
} 
Ma guardiamo ora un po'più a nord, nel territorio ticinese che nel Quattrocento faceva parte del Ducato. ${ }^{16}$ L'uso scritto del volgare in Ticino è testimoniato a partire dal XV secolo in vari tipi di testi di carattere pratico (amministrativo, giuridico, epistolare, notarile). ${ }^{17}$ La documentazione tarda accomuna quindi l'area ticinese a Milano e a quasi tutta la Lombardia, dove, fatta eccezione per Mantova, si registra la carenza di testi pratici in volgare almeno fino ai primi decenni del Quattrocento. ${ }^{18} \mathrm{Si}$ possono individuare alcuni elementi che hanno condizionato le vicende linguistiche del territorio e ne hanno determinato la specificità: l'essere terra di frontiera e di passaggio, fondamentale per importanza economica e strategica (con il ruolo chiave assunto da Bellinzona e da altri centri, come Lugano e Locarno), dunque "porta» tra il mondo tedesco e quello italiano, aperta alla pluralità culturale e linguistica; d'altra parte la situazione politica e amministrativa di appartenenza al ducato di Milano sotto i Visconti e gli Sforza, l'essere dunque terra lombarda a tutti gli effetti. Poi la particolare posizione geografica: l'essere cioè, da un lato, area periferica rispetto al centro

\footnotetext{
${ }^{16}$ Una versione più ampia di questo mio primo sondaggio apparirà in un volume di Storia del Ticino a cura di Giuseppe Chiesi e Paolo Ostinelli.

${ }^{17}$ Indispensabile il rinvio a Sandro Bianconi, I due linguaggi. Storia linguistica della Lombardia svizzera dal ' 400 ai nostri giorni, Bellinzona, Edizioni Casagrande, I989, pp. 25-34; Id., Lingue di frontiera. Una storia linguistica della Svizzera italiana dal Medioevo al 200o, Bellinzona, Casagrande, 200 I, pp. I 5-35; Ottavio Lurati, Il Canton Ticino, in L'italiano nelle regioni, a cura di F. Bruni, Torino, Utet, voll. 2, I 992-I994, vol I, pp. I45-48, vol. II (Testi e documenti), pp. I72-74. Sulle caratteristiche dei dialetti nel Ticino v. Id., Lombardia e Ticino, in Lexicon der Romanistischen Linguistik IV, a cura di Gerhard Holtus, Michael Metzeltin, Christian Schmitt, Tübingen, Max Niemeyer Verlag, I988, pp. 486-5 I6.

${ }^{18}$ Cfr. P. Bongrani - S. Morgana, La Lombardia, in L'italiano nelle regioni, cit., I992, I, pp. 84-I42, II, pp. I I I-70; Morgana, Storia linguistica di Milano, cit., passim; e cfr. Michele Colombo, Una confessio in volgare milanese del I3II, «Studi Linguistici Italiani», 36 (2010), pp. 3-26.
} 
amministrativo e politico del ducato, verso cui convergeva culturalmente anche per i modelli di lingua scritta. È significativo che i pochissimi frammenti poetici superstiti di area ticinese siano scritti dal segretario del comune di Bellinzona, Pietro Varrone, sui verbali comunali (I 460 circa). Si tratta di reminiscenze dantesche e guinizelliane («Vidi e chognobe lonbra de cholui / che per viltà fece il gran refuto»; «Amor che fa zentil ognia natura»), e di pochi versi che utilizzano tematiche e armamentario poetico tradizionale:
Revelie il verde de verde l'amore de verde reverdisce il mio inteleto per questo verde vedo per efeto che verde veste ognia zentil chore. Alegra il verde ciascun bel colore per verde ognia $\left[\ldots . .{ }^{19}\right.$

Certo troppo poco per ricostruire un quadro di influenze letterarie, ma sufficiente per testimoniare la circolazione e la fortuna della tradizione poetica toscana nei ceti colti bellinzonesi.

Anche nelle scritture di carattere pratico del Ticino si possono riconoscere gli elementi linguistici caratteristici della koinè cancelleresca milanese, di cui va sottolineata la funzione di modello di grande prestigio: esso viene usato infatti non solo nei documenti di cancelleria e nelle scritture pubbliche ufficiali, ma in tutte le scritture di carattere pratico, anche al di fuori dell'àmbito cancelleresco. I testi possono mostrare comunque notevoli differenze: esiste cioè una variabilità "verticale», di tipo diafasico, legata ai diversi usi e agli àmbiti funzionali di impiego del volgare (usi formali e ufficiali più controllati, usi informali e privati, familiari e meno sorveglia-

\footnotetext{
19 Sui frammenti letterari bellinzonesi cfr. Giuseppe Pometta, Vagiti del volgare a Bellinzona, "Educatore della Svizzera Italiana», I I 2 (I970), 3, pp. I 2-I 3 .
} 
ti), e una variabilità diastratica legata alla diversa competenza linguistica degli scriventi e alla loro formazione culturale. Occorre poi tener presenti le differenze «orizzontali», areali, che caratterizzano il processo di sprovincializzazione e italianizzazione delle scritture lombarde, per cui in zone geograficamente appartate e periferiche si possono mantenere a lungo usi linguistici più conservativi e connotati in senso locale in modo molto evidente.

Per la storia linguistica del Ticino quattrocentesco l'osservatorio privilegiato è costituito dal corpus di scritture provenienti dall'àmbito dell'amministrazione ducale e pubblicati nei monumentali volumi Ticino Ducale (d'ora in poi TD). ${ }^{20}$ L'adozione del volgare, che è attestata dalla metà secolo (il memoriale del conte Franchino Rusca, del marzo I 450, destinato probabilmente all'uditore ducale), ${ }^{21}$ riflette la prassi già in uso nella cancelleria milanese a partire dall'epoca viscontea (I 426) e la progressiva espansione del volgare, a scapito del latino. La base documentaria più ricca e significativa è rappresentata dalla corrispondenza ufficiale con Milano di cancellieri e funzionari ducali distribuiti nei centri nevralgici della regione ticinese e accomunati da una medesima formazione linguistica e culturale. Citiamo come esempio la lettera del

\footnotetext{
${ }^{20}$ I documenti finora pubblicati, che riguardano i territori ticinesi appartenenti in età sforzesca al Ducato di Milano e terre confinanti (Mesolcina, Valtravaglia, Valle d'Intelvi, Leventina, feudi dei Rusca), conservati nell'Archivio di Stato di Milano, sono compresi nei seguenti volumi: Giuseppe Chiesi - Luciano Moroni Stampa, Ticino Ducale, vol. I/I, Bellinzona Casagrande, I 993 (Francesco Sforza, I 450-I455); vol. I/II, ibid., I 994 (Francesco Sforza, I456-I46I); vol. I/III, ibid., I 995 (Francesco Sforza, I 462-66); G. Chiesi, Ticino Ducale, vol. II/I, ibid., I 999 (Galeazzo Maria Sforza, I 466-I 468); vol. II/II, ibid., 200 I (Galeazzo Maria Sforza, I 469I 472); vol. II/III, ibid., 2003 (Galeazzo Maria Sforza, I473-I476); vol. III/I, ibid., 2005 (Galeazzo Maria Sforza, I 476-I 477); vol. III/II, ibid., 20 Io (Galeazzo Maria Sforza, I 478).

${ }^{21} T D \mathrm{I} / \mathrm{I}, \mathrm{p} . \mathrm{I} 6$.
} 
commissario di Bellinzona Branda Pusterla al duca di Milano, per informarlo delle minacce rivolte dagli Svizzeri alla comunità di Locarno (6 settembre I468):

Illustrissime ac invictissime princeps et domine domine mi colendissime, humilima ac devota recomendatione premissa. Per una mia data a' tri del presente scripse ad vostra excellentia como quisti de la Ligha haveveno fate paci cum il dux de Ostorich et che erano tornati a casa molto alegri et arditi. Da poy soni tornati, vociferaveno de volere fare guerra ad le terre de prefata vostra excellentia, et precipue a Locarno, et che el sia vero, in questa sera il locotenente et il potestate d'esso Locarno me àno mandato una certa diffidanza mandata de quele parte pur in lingua todescha assay inepta la quale ò fato translatare in lingua nostra al meglio ò saputo e poduto, de la quale ne la mando la copia introclusa a vostra excellentia. ${ }^{22}$

Oltre a documentare la sostanziale identità di modelli linguistici con le scritture uscite dalla cancelleria milanese, questa lettera riveste un'importanza notevolissima, per la coscienza linguistica dello scrivente, che attribuisce un suo ruolo e una sua dignità al volgare, identificato come lingua del Ticino ducale (lingua nostra) in opposizione al tedesco della Lega svizzera. In alternanza col latino la lingua nostra comincia a essere adottata anche nelle lettere e nelle suppliche, che le comunità locali inviano al potere centrale. Citiamo come esempio la supplica rivolta ai duchi dalla comunità di Iragna, assalita e devastata nel I 467 da leventinesi e tedeschi:

Ex quo, illustrissimi signori, pregamo vostre signorie che se degnano deffendere da questa forza et providere come se convene a la indempnitate de li subditi et stato vostro, alyter a nuy sarà necessario, come forzati, abandonare lo payse et andare vagabondi. ${ }^{23}$

\footnotetext{
${ }^{22} T D$ II/I, p. 6 Iо.

${ }^{23}$ Ivi, p. 280.
} 
Lo stesso volgare di tipo cancelleresco risulta impiegato, sia pure saltuariamente, anche in scritture di ecclesiastici, tradizionalmente più fedeli al latino - ad esempio nelle suppliche dei canonici di Bellinzona al duca del $\mathrm{I} 463^{24}$ o del prete Pietro Rusca alla duchessa del I $466^{25}$ - e in alcune lettere inviate da gentildonne, ${ }^{26}$ dettate verosimilmente al segretario. La maggior parte delle scritture di àmbito ducale testimonia l'impiego di una varietà colta di lingua: l'affiorare talvolta di ipercorrettismi e ipertoscanismi negli scriventi indica la volontà di allontanarsi dagli esiti linguistici locali e di conformarsi al modello toscano: es. respuosta 'risposta', con sovraestensione del dittongo toscano; coci 'così', reazione ipercorretta all'esito assibilato locale, dove $c$ intervocalica $>s$; spigle 'spie', altra reazione ipercorretta all'esito locale palatizzato, tipo palea > paja, tosc. paglia. Non mancano tuttavia esempi di una varietà diastratica più bassa e conservativa di lingua, usata da scriventi meno colti; ne è un esempio la lettera di Antonio Brocchi da Lugano al duca ( 1465 ), da cui traspare una minore padronanza testuale e sintattica:

Unde non hè possibile che me possa partire cozì presto che ogi hè lo mercato qua a Lugano e sono caneparo de questa comunitade che ho da rezevere dinari da molte persone per diverse caxone. ${ }^{27}$

Il corpus documentario ducale è caratterizzato, sotto il profilo diafasico, da un registro linguistico prevalentemente formale, ufficiale: tuttavia in alcune lettere del carteggio è possibile riscontrare il passaggio a un registro più informale, evidenziato dall'uso di una sintassi più sciolta e di locuzioni colloquiali:

\footnotetext{
${ }^{24} T D$ I/III, p. 2 I 2.

${ }^{25} T D$ II/I, p. I 44.

${ }^{26} T D \mathrm{I} / \mathrm{II}, \mathrm{pp} .2$ I 5 e 360.

${ }^{27} T D \mathrm{I} / \mathrm{III}, \mathrm{p} .405$.
} 
Sono cum molti debiti a le spale, ${ }^{28}$ io ho pur tenuto uno puoco la briglia in mano $[\ldots]$ stentariano como cani, ${ }^{29} \mathrm{mi}$ strozaria mia isteso, ${ }^{30}$ vegnerò a quatro gambe se no' porò venire cum due. ${ }^{31}$

L'espressività colloquiale traspare soprattutto nelle lettere in cui ci sono esempi di discorso riportato e si alternano discorso indiretto e frammenti di discorso diretto: ${ }^{32}$ si veda ad esempio la vivacità della conclusione in volgare di una lettera in latino scritta da Antonio Besana a Giovanni Molo (6 novembre I 453):

Sumus hic repleti optimis novis, sed quid sit veritas quidve veraciter credamus satis ambigimus; tu ergo certiores nos facito. Universa familia mea per milies te salutat. El Porrino ha uno poco de male per le varole; pur ogni volta che 'l sente le campane da festa bixognia che 'l crida alta voce: «duca, duca», che l'è uno piacere. Habia al core el tuo Antonio. ${ }^{33}$

Di particolare interesse sono infatti le poche lettere di carattere familiare presenti nel carteggio, come la lettera di Pasello Paselli, commissario ducale di Lugano, a suo padre Giorgio (23 ottobre I466), in cui l'uso del discorso indiretto e il passaggio al discorso diretto mostrano in trasparenza tratti di colloquialità e si accompagnano a una mimesi onomatopeica della gestualità:

\footnotetext{
${ }^{28} T D \mathrm{I} / \mathrm{I}, \mathrm{p} \cdot 304$.

${ }^{29} T D \mathrm{II} / \mathrm{I}, \mathrm{p} .343$.

${ }^{30}$ Ivi, p. 345.

31 TD I/II, p. 242.
}

32 Sul discorso riportato si veda Bice Mortara Garavelli, La parola d'altri. Prospettive di analisi del discorso, Alessandria, Edizioni dell'Orso, 1985. Sulla presenza di tratti di parlato nello scritto: Scritto e parlato. Metodi, testi e contesti, a cura di Maurizio Dardano, Adriana Pelo, Antonella Stefinlongo, Roma, Aracne, $200 \mathrm{I}$.

33 TD I/I, p. 282. 
Io li disi per che caxone heri sira lui non volse vignire da mi, lui me rispose che quella non era hora da vegnire da mi, e che lui non li aveva voluto vignire. [...]

E più Franchino Castagna, cusino de dicto Zorzo, dise a dicto Zorzo: "Como farai te? Bisognarà andare a Milano». Dicto Zorzo fé uno dom dom cum lo brazo e dise: «O Milano o no». ${ }^{34}$

Le scritture ticinesi di carattere privato sono molto scarse e frammentarie: alcune lettere (da Aquila, I482; Tremona, I499, Lugano, I 493), una carta di conti (Carona, I 492) e una ricevuta di pagamento (Lugano, I493) non bastano per delineare un quadro di usi privati di scrittura, ma confermano comunque la diffusione del modello cancelleresco milanese anche in questi tipi di testo. ${ }^{35}$ Molto più consistente è invece la documentazione di carattere pubblico e ufficiale. Di grande importanza sono gli statuti delle comunità locali, che documentano il progressivo imporsi del volgare anche per gli usi giuridici e legislativi, prima dominio esclusivo del latino. Sono pervenuti in testo bilingue, latino e volgare, gli statuti di Centovalli (I 450), Carona (I 470) e Sonvico (I 473). ${ }^{36} \mathrm{Il}$ più antico, lo statuto di Centovalli, ${ }^{37}$ scritto su carta di produzione lombarda, mostra una forte commistione tra volgare e latino: "non vada [...] a pascolare in vineis in campis nec ortis in toto terratorio de Cenvaglie», dove Cenvaglie 'Centovalli' ricalca il toponimo locale Scenvài. Sono presenti lombardismi grafici e fonetici (ad esempio ughe 'uve', giande 'ghiande',

${ }^{34} T D$ II/I, p. I 37.

35 Sulle poche scritture private ticinesi quattrocentesche Bianconi, I due linguaggi, cit., p. 26; Id., Lingue di frontiera, cit., pp. 25-26.

${ }^{36}$ Per gli statuti volgari di comunità locali cfr. "Archivio Storico Ticinese», 93-94 (1983), pp. 258-83 (statuti di Carona), e pp. 284-304 (statuti di Sonvico); Romano Broggini, Statuti volgari e latini della comunità di Centovalli, «Verbanus», I4 (I993), pp. 59-Io9 (testo, studio storico-linguistico e glossario).

${ }^{37}$ Broggini, Statuti volgari e latini, cit., pp. 59-I09. 
dagno 'danno', pagni 'panni', husgi 'usci', masgio 'maschio', prede 'pietre'), e molti regionalismi in forma più o meno italianizzata, con le solite oscillazioni: camparo / campé 'sorvegliante dei campi', lavoradi / lavoré 'campi coltivati' (anche con una glossa esplicativa del termine locale: lavoré zoè in li prati), trozo 'sentiero sassoso', valegia 'piccola valle', biusare, ruscare 'pulire il bosco'. Numerosi i tecnicismi legati al diritto locale e alla regolamentazione dello spostamento del bestiame verso e dall'alpe (cargare e scargare, cioè 'caricare e scaricare l'alpe') e all'uso dei diritti di pascolo: giodenda 'pascolo chiuso da recinto', traso 'pascolo libero', trasare 'lasciar libero pascolo', tensa 'divieto di pascolo', tensare 'imporre la tensa', vacarisci 'diritti di pascolo', rodegare 'portare al pascolo a turno (roda)', pasturgheza 'funzione del pastore sull'alpe', fodro 'imposta in natura', guasti 'pascoli protetti', piadezare 'sporgere denuncia' ecc.

Appartengono invece agli ambienti della religiosità popolare $^{38}$ gli statuti della confraternita dei Disciplinati di Santa Marta di Daro, presso Bellinzona, trascrizione del testo dell'omonima confraternita di Como:

${ }^{38}$ Sul peso linguistico della devozione popolare e delle confraternite, vd. F. Bruni, Appunti sui movimenti religiosi e il volgare italiano nel QuattroCinquecento, "Studi Linguistici Italiani», 9 (1983), pp. 3-30. Per l'area ticinese Carlo Salvioni, Statuti della confraternita dei disciplinati di S. Marta di Daro, "Bollettino Storico della Svizzera Italiana», 26 (I 904), pp. 8 I-9I, ora in C. Salvioni, Scritti linguistici. Volume primo, Saggi sulle varietà della Svizzera italiana e dell'Alta Italia, a cura di Michele Loporcaro, Lucrezia Pescia, Romano Broggini, Paola Vecchio, Bellinzona, Edizioni dello Stato del Canton Ticino, 2008, pp. 562-73; sulle laudi dei Battuti di Santa Marta: Angelo Stella, Lombardia, in Storia della lingua italiana, a cura di Luca Serianni e Pietro Trifone, Torino, Einaudi, I994, voll. 3, vol. III, p. I96; Bruno Bordoni, Lugano. L'arciconfraternita della Buona Morte ed Orazione sotto il titolo di Santa Marta e di San Salvatore, Bellinzona-Lugano, Grassi IET, I97I, pp. I05 sgg. 
Quisti sono li capituli li quali deno seruare li deuoti de la disciplina e de la scola de madona sancta marta che sono fati per loro saluatione zoe de la comunita de le persone de la dita scola che stano in la cita de como. ${ }^{39}$

La veste linguistica notevolmente toscanizzata (ad esempio negli esiti chiama, dicono, voiamo, suo) e la patina solo debolmente settentrionale (ad esempio in forme come quili, rezevere, azo, lezuti, ognialonia) sembrerebbero indicare una datazione non anteriore alla metà del xv secolo. ${ }^{40}$ Gli statuti dei Disciplinati documentano comunque non solo la diffusione degli stessi modelli linguistici a Como e nelle valli ticinesi, ma la circolazione anche orale di una lingua sovraregionale, diversa dalle parlate locali, attraverso la pratica della lettura comunitaria degli statuti. Un articolo specifico ordina infatti esplicitamente che gli statuti siano letti una volta al mese nella scuola di Santa Marta:

XXI. Como li deuoti si deno odire ognia mexe li statuti una fiada per la sua salute.

Anchora ordenemo che azo che li conpagnioni non se domentagano quelle cosse che sono scrite per la sua discretione in li statuti che debieno essere lezudi in la scola de la disciplina onia mexe una fiada. ${ }^{41}$

La devozione popolare è stata anche per altri versi un canale importante di diffusione di modelli linguistici sovraregionali, «itineranti», non esclusivamente per via scritta: la presenza delle confraternite laiche dei Disciplinati o Battuti, che dall'Umbria e dalla Toscana si erano diffusi nell'Italia settentrionale, significa infatti che anche nell'area ticinese cir-

\footnotetext{
${ }^{39}$ Salvioni, Statuti, cit., p. 564 [82].

${ }^{40}$ Stella, Lombardia, cit., p. 196.

${ }^{41}$ Salvioni, Statuti, cit., pp. 567-68 [8 5-86].
} 
colavano preghiere e laudi che venivano cantate durante le processioni. È noto il codice dei Battuti di santa Marta di Como (circa I 420), ${ }^{42}$ i cui statuti, come s'è visto, servirono da modello a quelli di Daro, che contiene preghiere volgari e laudi di varia provenienza, tra cui una lauda jacoponica ("Quando t’alegri») e il Pianto della Vergine di Enselmino da Montebelluna: la lingua è composita e stratificata, e sotto la patina settentrionale sono riconoscibili componenti mediane, umbre e toscane. Ecco l'invocazione finale della laude di santa Marta, che doveva essere rivolta alla santa dal singolo devoto e dalla processione dei confratelli flagellanti:
E jesu cristo per mi prega
e per li toi servi desta scola
vestiti mo di bianca stola
santa marta nostra matrona
prega lo segnor kal ne perdona
santa marta nostra matrona
prega lo segnior kal ne perdona. ${ }^{43}$

Va poi ricordato che a Lugano la scuola di Santa Marta organizzava la Giobia santa, una rappresentazione dei misteri e della Passione collegata alla processione del giovedì santo: come le laudi, anche il teatro religioso di piazza era un importante canale di divulgazione di una lingua poetica sovraregionale.

Il peso della religiosità popolare nella storia linguistica del Ticino si può intravvedere anche nei frammenti quattrocenteschi superstiti in varie chiese di scritture esposte ai devoti (iscrizioni di San Pietro a Sonvico, di Santa Maria della Misericordia di Ascona; commenti didascalici a cicli pittorici). ${ }^{44}$ Largomento meriterebbe uno studio approfon-

\footnotetext{
${ }^{42}$ Stella, Lombardia, cit., pp. I94-95; Bordoni, Lugano, cit., pp. I05 sgg.

43 Bordoni, Lugano, cit., p. I 50.

${ }^{44}$ Sulle scritture volgari esposte nelle chiese ticinesi: Giovanni Rovelli,
} 
dito, soprattutto riguardo alla circolazione di libri devoti di diffusione interregionale e all'uso del volgare nella predicazione degli ordini religiosi, Umiliati e Mendicanti, documentata già dal xiv secolo a Milano, Como e Lugano con la fondazione di chiese e conventi: è nota fra l'altro la precoce diffusione in territorio ticinese del culto popolare per Bernardino da Siena. ${ }^{45}$

L'individualità linguistica del Ticino appare contrassegnata già dall'epoca medievale dal plurilinguismo, favorito dall'essere l'area ticinese "terra de passo e a le confine» ${ }^{46}$ tra il mondo tedesco e quello italiano, e dunque esposta a una molteplicità di rapporti e scambi che implicavano il contatto tra lingue diverse, con fenomeni conseguenti di interferenza lessicale tuttora presenti nelle parlate dialettali. ${ }^{47} \mathrm{E}$ anco-

La castellanza di Sonvico, Massagno, Tipografia S. Agostino, I927, p. 74; Virgilio Gilardoni, I monumenti d'arte e di storia del Canton Ticino. II/I. L'alto Verbano; Il circolo delle Isole (Ascona, Ronco, Losone e Brissago), Basel, I979, p. I28; Pittura a Como e nel Canton Ticino dal Mille al Settecento, a cura di Mina Gregori, Milano, I994, pp. 273-74 (su cui Bianconi, Lingue di frontiera, cit., p. 24).

45 Sulla fondazione di chiese: G. Pometta, Briciole di storia bellinzonese, Bellinzona, I977, serie X, II, pp. 464 sgg. Sull'uso del volgare nella predicazione degli ordini mendicanti cfr. Chiesi, Bellinzona ducale, cit., pp. I 82 sgg.; P. Ostinelli, Il governo delle anime. Strutture ecclesiastiche nel Bellinzonese e nelle Valli ambrosiane (XIV-XV secolo), Locarno, Dadò, I 998, pp. 275 sgg. Sul culto per san Bernardino da Siena cfr. Pometta, Briciole, cit., II, pp. 57 I sgg.; e ancora Ostinelli, Il governo delle anime, pp. 276-77.

${ }^{46} T D$ I/I, p. I 28. Lettera di Antonio Besana, commissario e podestà di Bellinzona, al duca di Milano: «Per tanto, illustrissimo signore mio, per fare de novo il devere mio verso vostra signoria, gli ricordo che questa terra non sta bene cusì senza qualche soldati, siando terra de passo e a le confine» (Bellinzona, 27 novembre I45 I).

47 Sull'immigrazione a Bellinzona cfr. Chiesi, Bellinzona ducale, cit., pp. 39 sgg. Sul plurilinguismo e sull'emigrazione in Ticino cfr. S. Bianconi, Lingua parlata uguale dialetto? Qualche ipotesi sulla situazione della Svizzera italiana nel Cinquecento e nel Seicento, "Archivio Storico Ticinese», 97 
ra il prezioso corpus di scritture ducali a offrire una variegata documentazione circa le occasioni di incontri plurilingui, come il passaggio di truppe e soldati mercenari tedeschi, ${ }^{48}$ la presenza di mercanti e di traffici commerciali («a la fera proxima de sancto Bartholomeo che se farà a Birinzona gli sarano molti mercadanti da Lozera» (I 454); ${ }^{49}$ "ge vegnarano [alla fiera di Bellinzona] de molti Thodeschi et Lombardi a modo consueto" (I 458); $;^{50}$ e perfino il transito di studenti mandati nelle Università italiane («alogiate in la vostra terra de Berinzona uno studente che andava secundo diceva luy a Padua» (1467). ${ }^{51}$ Esigenze politico-amministrative dovevano richiedere la pratica del bilinguismo o comunque la presenza di interpreti bilingui negli ambienti dei funzionari ducali a contatto con la Lega svizzera; così per esempio Antonio Besana riferiva al duca di un colloquio avuto con lo scoltetto ${ }^{52}$ e i consiglieri di Lucerna (I 463):

Ogi sono stati a disnare a caxa mia con mi quisti signori Lucernexi, cioè li sculteti et li megliori del loro consiglio secreto, et disnando lo primo parlare che hano fato è stato questo, che mo' mo' hano hauto litere da suoi ambasiatori che erano apè del re de Franza. ${ }^{53}$

E così Giacomo da Trezzo riferiva a Cicco Simonetta di un colloquio privato avuto con un mercante lucernese di passaggio:

Et trovandose qui in Birinzona uno Antonio Sceler, citadino et

(I984), pp. 2I-32 (in riferimento ai conti della compagnia di Brissago).

${ }^{48} T D \mathrm{I} / \mathrm{I}$, pp. 258-59.

49 TD I/I, p. 335.

${ }^{50} \mathrm{TD}, \mathrm{I} / \mathrm{II}, \mathrm{p} .2 \mathrm{I} 7$.

51 TD II/I, p. 479

${ }^{52}$ Lo scoltetto (ted. Schultheiss) o magistrato (borgomastro) della città.

53 TD I/III, p. 230. 
merchadante de Lucera [...] trovandose a mangiare cum mi me ha dito in secreto che 'l duca de Savoya è quello che ha fatto instancia de mandare al prefato nostro illustrissimo signore li dicti ambasadori. ${ }^{54}$

Oltre al contatto con il mondo di cultura e lingua tedesca, l'altro importante fattore di plurilinguismo è dato dalle prime correnti migratorie verso altre regioni italiane, che preannunciano i più massicci spostamenti dei secoli successivi (ma non è da sottovalutare nelle sue conseguenze linguistiche anche la presenza a Bellinzona e in altri centri di famiglie di provenienza lombarda e da altre regioni). Ne offre una significativa testimonianza il Libro della Confraternita del SS. Crocifisso di Brissago nella città di Fiorenza, che contiene le registrazioni di scriventi colti brissaghesi lì emigrati: le scritture, in volgare a partire dal primo Cinquecento, sono vivacemente plurilingui e si caratterizzano per la compresenza di elementi locali (disnà, canavé) e della koinè cancelleresca (prandio, canepario) con quelli toscani (colazione, camerlingo). ${ }^{55}$ Non meno importante è la documentazione relativa a una famiglia di fornaciai biaschesi emigrati a Volterra ai primi del '500:

A dì ultimo di luglio I 508 .

Giovanni mio nipote dé dare a dì soprascritto per uno mantello et per uno cintolo e per un paio di scharpette d'achordo ogni cosa somma lb. I I s. $2 .{ }^{56}$

Il quaderno di conti di Giovan Battista di Biasca (I 506I 508), che annota "gli è vera cosa che i’ ò chominciato ampare ('imparare') a scrivere da Nicholaio di Francescho Borrani-

\footnotetext{
${ }^{54} T D \mathrm{I} / \mathrm{I}, \mathrm{p} .334$.

55 Bianconi, Lingua parlata, cit., passim; I due linguaggi, cit., pp. 32 sgg.; Lingue di frontiera, cit., p. 34.

${ }^{56}$ Bianconi, Lingue di frontiera, cit., p. 32.
} 
ni da Volterra», ${ }^{57}$ testimonia infatti la conquista della nuova condizione di alfabetizzato, e attesta «l'avvenuta integrazione linguistica dell'emigrato nella cultura e nella lingua del luogo di emigrazione». ${ }^{58} \mathrm{Si}$ intravedono gli sviluppi sociolinguistici legati all'emigrazione ticinese dell'età moderna, in particolare l'estensione dell'alfabetismo e della scrittura anche agli illetterati, che costituirà uno degli elementi caratterizzanti e decisivi nella storia linguistica dei baliaggi dopo il distacco dal ducato di Milano. ${ }^{59}$ Siamo, ormai, alle soglie delle importanti trasformazioni che avvieranno anche in Ticino, ma in modi e tempi diversi rispetto all'area milanese e lombarda, il lento e discontinuo percorso verso l'italiano scritto e parlato. ${ }^{60}$

\footnotetext{
${ }^{57}$ Bianconi, I due linguaggi, cit., p. 34.

${ }^{58}$ Bianconi, Lingue di frontiera, cit., p. 33.

59 Ibid.

${ }^{60}$ Sul passaggio dall'oralità alla scrittura e sull'italiano dei semicolti, principalmente: G. R. Cardona, Culture dell'oralità e culture della scrittura, in Letteratura italiana Einaudi, a cura di A. Asor Rosa, II, Torino, Einaudi, I983, pp. 25-Ior; Bruni, L'italiano, cit., pp. 343 sgg.; Paolo D’Achille, L'italiano dei semicolti, in Storia della lingua italiana, II, pp. 4I-79; S. Bianconi, L'italiano lingua popolare. La comunicazione scritta e parlata dei "senza lettere" nella Svizzera italiana dal Cinquecento al Novecento, Firenze-Bellinzona, Accademia della Crusca-Edizioni Casagrande, 20 I3; EnricoTesta, Litaliano nascosto. Una storia linguistica e culturale, Torino, Einaudi, 20I4.
} 



\section{Francesco Bruni}

FRA TOSCANI E LOMBARDI

(CON UNA SECONDA SCHEDA SU «FLORENTINITAS»)

1. Per introdurre l'argomento di questo contributo dedicato a un aspetto, tra i molti, che Maurizio Vitale ha indagato e continua a indagare nella sua lunga, feconda carriera di studioso, mi soffermerò sinteticamente su un episodio della nuova filologia e linguistica nata in Italia all'indomani dell'Unità politica, e mostrerò come, in questo caso (non diversamente da altri, estranei all'argomento delle pagine che seguono), Vitale abbia fatto avanzare le conoscenze su alcuni problemi fondamentali che furono affrontati dai pionieri di questi studi.

Prendo le mosse da uno dei cinque romanisti nominati, dopo l'Unità, in altrettante Università del Regno, Francesco D'Ovidio, e in particolare dal suo libro Le Correzioni ai «Promessi sposi» e la questione della lingua ( I 893).

Il secondo dei due argomenti enunciati da questo titolo traccia uno dei primi bilanci sulla questione della lingua e la storia della questione, cui è dedicato il capitolo intitolato «Un po’ di discussione teorica e di esposizione storica della questione della lingua».

A proposito della fase cinquecentesca, durante la quale la questione della lingua sorse e fu discussa ampiamente, per continuare poi con intensità variabile nei secoli seguenti, D'Ovidio osserva, con un giudizio tuttora valido circa il dualismo toscano-lombardo e la disponibilità dei centri meridionali al fiorentino-toscano:

le controversie furon quasi interamente o fra toscani e lombardi (nome che allora aveva un senso men circoscritto potendo accennare a tutta l'Italia superiore alla Toscana), benché i secondi combattessero per lo più in nome della lingua usata in corte di Roma, 
ovvero tra fiorentini ed altri toscani; ma il Mezzogiorno fu quasi scevro di ribellione. Il nobilissimo Sannazaro, riverito e additato ad esempio da toscani e non toscani, era nella più cordiale intelligenza con tutti. ${ }^{1}$

L'interesse per la storia della questione della lingua nasceva dall'attualità, dal risorgere cioè della questione nei termini nuovi proposti dalla soluzione fiorentina indicata dalla teoria e dalla prassi narrativa di Manzoni non meno che dalla politica scolastica postunitaria, e dalla forte critica mossa a Manzoni e alla sua scuola da Graziadio Isaia Ascoli. La discussione aveva una rilevanza scientifica, nel clima della nuova linguistica storica che in Italia cominciava ad avere pochi ma validi rappresentanti, tra i quali era D'Ovidio. Questi era partito, per sua stessa dichiarazione, da una posizione di «manzoniano arrabbiato»: sono parole che si leggono in un articolo intitolato Lingua e dialetto, nel quale l'autore mostrava di aver modificato la sua posizione. Con Lingua e dialetto, infatti, uscito nel $1873,{ }^{2}$ D'Ovidio aveva formulato con grande prontezza una soluzione nuova sullo stimolo del Proemio

${ }^{1}$ Cfr. Le correzioni ai "Promessi sposi" e la questione della lingua. Terza edizione interamente rifusa per uso delle scuole, Napoli, Morano, I893, pp. I 5 I-52; poi nella quarta edizione, dallo stesso titolo, Napoli, Pierro, I 895, p. I 28 (di questa edizione, definitiva, si fece una ristampa postuma, Napoli, Guida, I933, come vol. vin delle Opere di Francesco D’Ovidio). Le citazioni sono dall'edizione del i 895 .

${ }^{2}$ Francesco D’Ovidio, Lingua e dialetto, nella neonata «Rivista di Filologia e di Istruzione Classica», I (I873), pp. 564-83; il lavoro fu ripubblicato con lo stesso titolo nei Saggi critici, Napoli, Morano, I878, pp. 437-65, e si può leggere anche nell'antologia degli Scritti linguistici, a cura di Patricia Bianchi, Introduzione di Francesco Bruni, Napoli, Guida, I982, pp. 4665 (secondo il testo del I878; le varianti apportate all'edizione del I873 sono registrate alle pp. I 53-56). L'espressione "manzoniano arrabbiato», caratteristica dello stile dovidiano, di natura accademica ma incline alla scioltezza saggistica, è all'inizio di Lingua e dialetto (Scritti linguistici, p. 46). 
di Ascoli (pubblicato, nello stesso I 873, nell' «Archivio Glottologico Italiano»), e avanzato una proposta conciliativa tra la tesi manzoniana e quella della nuova scienza linguistica di Ascoli.

In secondo luogo il problema, importante sul piano scientifico e intellettuale (necessariamente accessibile a pochi intendenti), aveva riflessi anche sulla collettività italiana, perché incideva sugli orientamenti della nuova scuola dell'Italia unita e della politica linguistica del giovane Regno, impegnato nell'alfabetizzazione obbligatoria della popolazione in età scolare e quindi nel potenziamento dell'istruzione elementare e postelementare.

Anche se non mancavano gli oppositori, a molti il romanzo di Manzoni appariva come un modello di prosa moderna da proporre nelle scuole della nazione: ma come un modello unico, o come un accesso privilegiato per invitare i giovani ad accostarsi anche ai classici della lunga tradizione letteraria italiana? E perché non invitare gli allievi a ripercorrere almeno in parte il lavorio correttorio dalla Ventisettana alla Quarantana in modo da indicare, ai tanti rimasti fino ad allora esclusi dall'istruzione scolastica, un esempio altissimo di conquista progressiva di una lingua moderna?

Conciliativo tra dottrina ascoliana e teoria e prassi narrativa manzoniana (quest'ultima riconosciuta pienamente da Ascoli nel suo valore altissimo), D'Ovidio passa contemporaneamente dalla discussione delle teorie linguistiche di Manzoni e Ascoli a un esame puntuale e rigoroso della prosa del romanzo e conduce un'analisi comparativa delle correzioni tra le due edizioni dei Promessi sposi. Nei Saggi critici del I 878 in cui ripubblica, con ritocchi, Lingua e dialetto del I873, include anche alcuni lavori inediti, uno dei quali, La lingua dei «Promessi sposi», ${ }^{3}$ è uno studio delle varianti, ricco di intel-

${ }^{3}$ Saggi critici, cit., pp. 539-602. 
ligenti valutazioni sul progresso artistico compiuto da Manzoni con l'approdo del i 840.

Vale la pena di seguire, ora, l'intreccio tra l'attività dovidiana su Manzoni e la lingua, e quella che, sugli stessi argomenti ma da un punto di vista un po' diverso, esercitò Luigi Morandi. Per seguire con chiarezza i loro punti di vista, che furono pubblicati su rivista, indipendentemente l'uno dall'altro, nel I 873, e che subito dopo dettero vita a una fitta serie di interventi lungo una franca, onesta discussione a base di repliche che rimbalzano da un libro all'altro, è utile tener sott'occhio la Scheda bibliografica qui di seguito proposta, che registra la successione degli scritti presi in esame o, almeno, menzionati.

I 873 D'Ovidio, Lingua e dialetto, «Rivista di Filologia e di Istruzione Classica» I, pp. 564-83.

I 873 Morandi, Di un pregiudizio letterario intorno $i$ "Promessi sposi», «Rivista Europea» 4, pp. 217-35 e 476-5II.

I 874 Morandi, Le correzioni ai "Promessi sposi» e l'unità della lingua. Lettera inedita di Alessandro Manzoni con un discorso di Luigi Morandi, Milano, Rechiedei.

Comprendono:

Lettera di Manzoni al Casanova (30 marzo I871), pp. 9-22;

Un pregiudizio letterario intorno $i$ "Promessi sposi». Discorso di Luigi Morandi, pp. 25-68;

Saggio comparativo della prima edizione dei «Promessi sposi» con la seconda, pp. 69-95 (il cap. xxxiv dei Promessi sposi è riprodotto dalle edizioni del I 827 e del I 840 su due colonne).

I878 D’Ovidio, Saggi critici, Napoli, Morano.

Comprendono:

Lingua e dialetto (1873), pp. 437-65;

La lingua dei "Promessi sposi», pp. 539-602.

I 879 Morandi, Le correzioni ai "Promessi sposi» e l'unità della lingua. [...] Terza edizione migliorata e molto accresciuta da poter servire anche alle scuole, Parma, Battei. 
Comprendono:

Lingua e dialetto (Risposta al prof. Francesco D'Ovidio), datato I 879, pp. I7I-209;

Pretesi e veri difetti di forma de' "Promessi sposi" (Parte seconda. Risposta al prof. F. D'Ovidio), datato I 879, pp. 26I-99;

Saggio comparativo della prima edizione de" "Promessi sposi» con la seconda, pp. 329-8o.

I880 D'Ovidio, La lingua dei "Promessi sposi» nella prima e nella seconda edizione. Seconda edizione, ad uso delle scuole ginnasiali e liceali, con varie Appendici, Napoli, Morano.

Comprende:

La lingua dei "Promessi sposi», pp. 2 I-95.

Appendice vi: Il libro del prof. Morandi, pp. I 5 I-233 (solo nell'indice, singolarmente: Critica del libro del Morandi, ed esame sommario della questione della lingua).

I 893 D'Ovidio, Le correzioni ai "Promessi sposi» e la questione della lingua. Terza edizione interamente rifusa per uso delle scuole, Napoli, Morano, con un'Avvertenza premessa alla terza edizione, datata settembre I 892 [la data si ricava dall'edizione del I 895], pp. VII-XIII.

I 895 D’Ovidio, Le correzioni ai "Promessi sposi" e la questione della lingua, Napoli, Pierro, con una Poscritta, datata ottobre I894, in coda all'Avvertenza riprodotta dall'edizione del I 893.

Luigi Morandi stampa nel 1879 Le correzioni ai «Promessi sposì e l'unità della lingua, ampliamento e aggiornamento di pubblicazioni precedenti, discute in Lingua e dialetto il saggio omonimo di D'Ovidio, in Pretesi e veri difetti di forma de' "Promessi sposi» risponde a La lingua dei «Promessi sposi», capitolo dei Saggi critici dovidiani. ${ }^{4}$ Morandi non accetta il

${ }^{4}$ Le correzioni ai "Promessi sposi" e l'unità della lingua, cit. Nella prefazione, Morandi cita come seconda l'edizione Rechiedei, I874. Il saggio Di un pregiudizio letterario era già apparso nella "Rivista Europea» 4 (I873), pp. 2 I 7-35 e 476-5 I I, da considerarsi come prima edizione. Il pregiudizio che Morandi intende dissipare è la convinzione della superiorità dell'edi- 
compromesso Manzoni-Ascoli proposto da D'Ovidio, gli rimprovera scarsa sensibilità per l'esigenza, centrale nella dottrina manzoniana, di un vocabolario del fiorentino-italiano, sostiene le ragioni dell'uso parlato di Firenze. Nel secondo studio critica alcune valutazioni di D'Ovidio troppo limitative della prosa narrativa e delle scelte di lingua manzoniane. Il dissenso è espresso con chiarezza, ma non mancano i punti d'incontro tra due studiosi entrambi sostenitori della lezione manzoniana.

Passa un altro anno e anche D'Ovidio scende in campo su un terreno divulgativo e scolastico, come aveva fatto Morandi con il volume del I 879: è del i 880 La lingua dei "Promessi sposi" nella prima e nella seconda edizione. Come si vede, il titolo riformula la prima parte del titolo di Morandi nell'edizione del 1879 (e già del I874). Nell'opera del I 880 confluiscono gli interventi di tipo militante, come Lingua e dialetto; lo studio dei Promessi sposi e della revisione linguistica della Quarantana; la proposta del romanzo come veicolo, però non esclusivo, della lingua nazionale nell'insegnamento scolastico; una critica "assai viva " (cioè vivace) all'opera di Luigi Morandi che, diversamente da D'Ovidio, era e restava un manzoniano ortodosso.

Nel I 893 D’Ovidio pubblica, e lo ristampa due anni dopo (cfr. le nn. I e 5), l' approdo ventennale di una discussione che rifonde aggiorna e modifica molti interventi precedenti. Il titolo è ora in parte conservato, in parte integrato: Le correzioni ai "Promessi sposi» e la questione della lingua: cambia il titolo originario ( I 878) e viene riprodotta la prima metà di

zione Ventisettana sulla Quarantana (come si sa, la Ventisettana continuò a essere ristampata a lungo, e a circolare tra lettori anche illustri e critici noti, poco sensibili all'innovazione della Quarantana).

${ }^{5}$ Così nell'Avvertenza premessa alla terza edizione del volume Le correzioni ai "Promessi sposi", che cito dall'edizione del I 895. 
quello di Morandi (1874 e I879): alla seconda metà del titolo di quest'ultimo, che era l'unità della lingua, D'Ovidio fa ora corrispondere una seconda metà, di suo conio: la questione della lingua. Lo studioso osserva che la sua tesi sulla «giusta conciliazione fra la dottrina manzoniana e le più o meno avverse, tra le quali capitalissima quella dell'Ascoli» si andava risolvendo «in teoria e ancor più in pratica»; tuttavia ritiene che non sia ancora giunto il momento in cui "la questione della lingua» possa considerarsi come un fatto puramente «retrospettivo e storico». ${ }^{6}$

La riedizione Morano I 893 e la ristampa Pierro si fondavano in gran parte sugli spogli del I880, ma furono ampiamente riscritte, pur senza cambiare il punto di vista sostenuto nei primi, più antichi interventi, come con giusta ragione osserva l'autore nella Poscritta:

Nel rifare l'edizione del 1880 io misi non poca cura e v'arrecai moltissimi mutamenti, non già nelle dottrine o nella qualità delle argomentazioni, ma per l'assetto della materia e pei tagli e le aggiunte fattevi; e ne risultò un libro sotto più rispetti nuovo, oltreché rinnovato da capo a fondo nella forma. Mi preme di notarlo, perché mi son dovuto accorgere come a più d'uno la cosa sia rimasta inavvertita, soprattutto per il capitolo dov'è delineata la storia della questione della lingua. ${ }^{7}$

Di particolare rilievo è per gli scopi di questo contributo l'ultima osservazione: se la seconda metà del titolo affiora solo nel I 893 e nel I 895, il motivo è che nelle Correzioni del I 880 sulla storia della questione della lingua c'è poco più che uno spunto di appena poche pagine, dedicate a una trattazione molto sommaria da Trissino a Monti: ${ }^{8}$ poche ma significati-

\footnotetext{
${ }^{6}$ Poscritta all'Avvertenza, nell'ed. I 895, pp. XIV e Xv.

${ }^{7}$ Le correzioni, cit., p. XIV.

${ }^{8}$ La lingua dei «Promessi sposi», I880, cit., pp. I88-96; solo dall'indice
} 
ve, perché nate al calore di un problema di critica militante, quale era la controversia sulla lingua tra Manzoni e Ascoli. In altre parole, dall'orientamento linguistico e scolastico da prendere nel presente e nel futuro prossimo, D'Ovidio fu indotto ad approfondire gli aspetti di quella questione come si era presentata nei secoli precedenti: l'attualità, insomma, era il punto di partenza, e non d'arrivo, dell'attenzione alla storia.

2. Il pensiero linguistico e critico di D'Ovidio di cui si è riassunta la storia, intrecciata con quella dei lavori di Morandi, interessa per due motivi. Il primo è che vi confluiscono due argomenti, l'analisi delle correzioni dalla Ventisettana alla Quarantana e la storia della questione della lingua, il primo dei quali, nella vasta produzione scientifica di Vitale, è stato oggetto di uno studio tanto breve quanto denso. ${ }^{9}$ All'altro polo, la questione della lingua, Vitale ha dedicato un'opera sistematica che, a distanza di trent'anni dall'edizione definitiva, resta fondamentale. ${ }^{10}$

Ora questi due argomenti, rampollati l'uno dall'altro, hanno in D'Ovidio e nella sua trattazione pionieristica il punto di partenza della moderna bibliografia scientifica. Al fine scrutinio variantistico compiuto da D'Ovidio si può solo accostare, in quegli anni, l'ottimo lavoro di Morandi; quanto alla parte riguardante la storia della questione della lingua, prima della trattazione di D’Ovidio si possono appena ricordare due lavori piuttosto modesti, di fatto con-

risulta il titolo che menziona la questione della lingua (si veda la scheda bibliografica di D'Ovidio I 895).

${ }_{9}$ M. Vitale, La lingua di Alessandro Manzoni, Milano, Cisalpino-Goliardica, 1986 (2.a ed., Milano, Cisalpino - Istituto Editoriale Universitario, I992).

${ }^{10}$ M. Vitale, La questione della lingua, Palermo, Palumbo, I984 (I.a ed. I960). 
temporanei o di poco anteriori. ${ }^{11}$ In altre parole, Vitale si è occupato, da par suo, di due questioni fondative (si potrebbe dire: dei fondamentali) dei nostri studi storico-linguistici, che in D'Ovidio hanno il loro capostipite. Per altri studi di Vitale si potrebbe richiamare l'origine prima, analogamente, negli studi di altri romanisti della prima generazione, cui appartenne D'Ovidio.

Il secondo motivo per la digressione su D'Ovidio sta nel felice spunto sulla polarizzazione lombardi contro toscani nella questione della lingua e nelle resistenze opposte dai primi al primato linguistico rivendicato dai secondi, mentre assai minori furono, nell'Italia centromeridionale, le resistenze al fiorentino-toscano. Anche a questo proposito, un episodio importante del dualismo linguistico di lombardi e toscani è stato studiato a fondo da Vitale nel 1985: Lombardi e toscani nella questione del Vocabolario. L'Istituto nazionale di scienze, lettere ed arti e l'Accademia della Crusca. ${ }^{12}$ Su alcuni momenti di questo dualismo, che attraversa in forme e modi diversi la lunga traiettoria dalle origini fino al Proemio di Ascoli incluso, vorrei soffermarmi in questa occasione, in omaggio al Maestro che si festeggia nell'occasione presente.

${ }^{11}$ Nella Bibliografia generale (La questione della lingua, cit., p. I3), prima del capitolo Un po' di discussione teorica e di esposizione storica della questione della lingua di D'Ovidio, nell'ed. del i 895, Vitale elenca solo Amedeo Crivellucci, La controversia della lingua nel Cinquecento, Sassari, Dessì, I 880 (circoscritto, come risulta dal titolo, al xvi secolo) e l'onesto lavoro, da Dante alla fine del xIx secolo, di Leone Luzzatto, Pro e contro Firenze. Saggio storico sulla polemica della lingua, Verona-Padova, Drucker, I 893. Il lavoro del Crivellucci (studioso noto soprattutto come storico) è ricordato da D’Ovidio, La lingua dei "Promessi sposi", I880, cit. p. I93, n. I, come «lavoro giovanile, abbastanza giudizioso e accurato». Una semplice menzione bibliografica di Crivellucci e Luzzatto è nell'ed. I 895, cit., p. I 24 n. 12 Il lavoro è stato ristampato nel volume La veneranda favella, Napoli, Morano, I988, pp. 489-563. 
3. Si sa che alla percezione del sé, tanto individuale che collettivo, contribuisce, in un intreccio nel quale sarebbe probabilmente illegittimo, oltre che difficile se non impossibile, distinguere a chi tocchi la mossa iniziale, la coppia dell'etero-, non solo dell'autopercezione (o autocoscienza). Non è il neonato che si attribuisce il nome proprio, e non diversamente le comunità sono non di rado designate dal vicino confinante: con il suo accento greco lo rivela la terra dei longobardi, la Lombardia, mentre la vicina Romagna (<Romània) con il suo accento latino (diversamente dalla Romanìa) è la terra dei Romàioi, dei Romani, cioè dei bizantini di lingua greca, rimasti in quella e in altre fasce della penisola anche dopo la conquista di gran parte dell'Italia continentale compiuta dai Longobardi.

In un capitolare Carlo Magno cede al figlio Pipino l'«Italia, quae et Lombardia dicitur», ${ }^{13}$ e molto più tardi Enrico di Crissey (prima metà del XIV secolo), commentatore dei Modi significandi di Jean Josse di Marville, propone, parlando di alcune lingue volgari, un'equivalenza tra Lombardi e Ytali$c i$ : «ydiomata multiplicia sunt apud Latinos, quia aliud est apud Gallos, aliud apud Germanos, aliud apud Lombardos seu Ytalicos». ${ }^{14}$ Vista dall'Europa settentrionale, da Oltralpe, la vicenda di Enea è modificata in vario modo nel Roman d'Eneas francese del XII secolo; l'eroe, tra l'altro, è detto originario della Lombardie (per es., vv. 339-342), sicché la

\footnotetext{
${ }^{13}$ Cito da Jacques Le Goff, L'Italia fuori d'Italia, in Storia d'Italia, a c. di Ruggiero Romano e Corrado Vivanti, II 2, Torino, Einaudi, I974, pp. I935-2248, a p. 1945, dove si trovano diverse interessanti variazioni sull'Italia come Italia settentrionale o sull'Italia distinta dalla Tuscia.

${ }_{14}$ Traggo la testimonianza da Gian Carlo Alessio, La cultura. Tradizione latina e origini romanze, in Manuale di Letteratura italiana, a cura di Franco Brioschi e Costanzo Di Girolamo, I, Torino, Bollati Boringhieri, I993, pp. 3-44, a p. 22.
} 
Lombardie, non l'Italia o il Lazio, è la sua terra promessa; ${ }^{15}$ e di essere additati come "questi lombardi cani» e perciò rapinati e forse trucidati hanno paura i due affaristi toscani che in Borgogna ospitano ser Ciappelletto (Decameron I I, 26). Tuttavia non mancano testimonianze più precise: gli Annales Stadenses del XIII secolo registrano all'anno I I 72 l'attività guerresca di «Christianus Mogontinus, archiepiscopus, imperialis aulae cancellarius et sedis apostolicae legatus, cum Brabantinis per Longobardiam et Tusciam omnia depopulans». Alla testa di truppe del Brabante il personaggio devasta l'Italia settentrionale lombarda e la Tuscia, con appropriata distinzione geografica alla quale corrisponde, nell'anno I I73, una nota sulla sua poliglottia: "Christianus archiepiscopus licet Teutonicus, scilicet Thuringus, disertus extitit et facundus, vir largus et illustris, utens lingua Latina, Romana, Gallica, Graeca, Apulica, Lombardica, Brabantina, uti lingua materna». ${ }^{16}$

Di qua dalle Alpi, il punto di vista è diverso e, inevitabilmente, più articolato. Dalla sua prospettiva lombarda, Salimbene de Adam racconta che nel 1285 morì un Barnaba da Reggio:

${ }^{15} \mathrm{Da}$ aggiungere alle molte, non sempre omogenee testimonianze raccolte da Gerhard Rohlfs nel suo breve contributo su Italia e Longobardia [I959], poi in Studi e ricerche su Lingua e dialetti d'Italia, Firenze, Sansoni, I972, pp. 3-5; di Rohlfs cfr. anche la Grammatica storica della lingua italiana e dei suoi dialetti, Torino, Einaudi, I966-I969, \$ I076. Inoltre si veda Francesco Bruni, Italia. Vita e avventure di un'idea, Bologna, Il Mulino, 20 Io, pp. 69-74; Ferdinando Raffaele, Raffigurazioni dell'Italia e dei suoi abitanti nelle chansons de geste (secc. XII-XIII), "Le Forme e la Storia», N.S., 3, I (2010), pp. 3I-43.

${ }^{16}$ MGH, SS., XVI, 347. Il problematico Romana difficilmente si riferisce a un idioma italiano, osservò giustamente Bruno Migliorini (Dante nella storia della lingua italiana, "Cultura e Scuola», I3-I4 (I965), pp. I38-43, a p. 138$)$. 
Hic multum fuit amicus meus. Et fuit solatium clericorum, canonicorum et omnium prelatorum, militum et baronum et omnium qui solatium requirebant, quantum ad verba sua. Nam optime loquebatur Gallice, Tuscice et Lombardice et aliis multis modis, scilicet qualiter pueri cum pueris pueriliter locuntur, qualiter mulieres cum mulieribus et cum commatribus suis familiari colloquio mutuo referunt facta sua. Et sciebat representare modum predicandi antiquorum predicatorum, secundum quod predicabant tempore Alleluie, quando intromittebant se de miraculis faciendis, ut diebus illis oculis meis vidi. ${ }^{17}$

Con la versatilità della mimica e della parola, il Barnaba da Reggio tratteggiato da Salimbene e intrattenitore ricercato da ecclesiastici e laici di condizione civile o militare aveva, come si vede, una doppia specialità: era un bravo imitatore e sapeva contraffare il linguaggio infantile e il chiacchiericcio e i pettegolezzi delle donne quando parlano tra loro e, passando dal linguaggio quotidiano dei bambini e delle donne (parlanti diversi dai maschi adulti) all'oratoria più enfatica, scimmiottava l'eloquenza dal pulpito dei predicatori vecchia maniera. In secondo luogo, Barnaba conosceva tre lingue, il francese, il toscano e il lombardo, sentite come distinte l'una dall'altra: il toscano è percepito come diverso e lontano dal lombardo non meno che dal francese. Benché altrove Salimbene lodi lo spirito comico dei fiorentini con riferimento specifico al loro idioma (ma si resta all'interno della pura oralità), per lui non si è ancora formato al di sopra della ricchezza idiomatica delle molte varietà linguistiche locali quel tetto italiano che trova la sua prima teorizzazione compiuta ed esplicita nel De vulgari eloquentia.

Non diversamente l'autore del cosiddetto Sirventese lombardesco si prova a comporre in lombardo, prendendo come pietra di paragone (e di eteropercezione, nella fattispecie di eteroglossia) il provenzale:

${ }^{17}$ Salimbene de Adam, Cronica, ed. Giuseppe Scalia, Bari, Laterza, I966, II, p. 864 . 
Poi qe neve ni glaza non me pot far guizardo, e qe dolzamentr'ardo en l'amor qe m'abraza, ben è rason q'eo faza un sirventés lombardo, qé del proenzalesco no m'acresco: - e fòra cosa nova, q'om non trova - sirventés lombardesco; ${ }^{18}$

cioè, nella parafrasi proposta da Alfredo Stussi:

Dato che né neve né ghiaccio non mi possono indebolire e dato che dolcemente ardo nell'amore che mi abbraccia, è giusto ch'io faccia un serventese lombardo poiché del serventese provenzale non posso glorificarmi: e sarebbe una novità, poiché non si compone serventese lombardo. ${ }^{19}$

Lombardo è in rima con guizardo; lombardesco (suffissato come latinesco o tedesco o francesco) è in rima semanticamente distintiva con proenzalesco: idioma in cui l'anonimo non intende comporre, scegliendo la novità (intesa positivamente o negativamente, come un ripiego?) del lombard(esc)o.

Invece una novella del Novellino (LXXxiv) è giocata su un equivoco linguistico nella Lombardia e nella Marca Trevigiana, secondo una prospettiva spiegabile dall'osservatorio toscano dell'anonimo autore della raccolta, il quale mette a

${ }^{18}$ Cfr. Alfredo Stussi, Note sul sirventese lombardesco, "Cultura Neolatina» 60 (2000), pp. 28I-3I0, a p. 294; cfr. anche Maria Luisa Meneghetti, "Vidas" e "Razos": sondaggi di stratigrafia funzionale (con una riflessione su fonti e significato del "Sirventes lombardesco»), nel volume I trovatori nel Veneto e a Venezia (Atti del Convegno Internazionale, Venezia 28-3 I ottobre 2004), a cura di Giosuè Lachin, Roma-Padova, Antenore, 2008, pp. 227-5I: la Meneghetti accosta al Sirventes un testo provenzale e svolge considerazioni, peraltro non decisive, sulla sua possibile attribuzione a Sordello (pp. 24I-5I).

${ }^{19}$ Stussi, Note, cit., p. 294. 
confronto toscano e lombardo. In una serie di episodi centrati su Ezzelino da Romano si legge questo aneddoto:

In Lombardia e nella Marca si chiamano le pentole ole. E la sua famiglia [di Ezzelino] aveano un dì preso un pentolaio per maleveria, e menandolo a giudice, messere Azzolino era nella sala; disse: "Chi è costui?» L'uno rispuose: «Messere, è un olaro». "Andalo ad impendere». "Come, messere, ch'è un olaro?» «E però dico che voi l'andiate ad impendere». "Messere, noi diciamo ch'elli è un olaro». «E ancor dico io che voi l'andiate ad impendere». Allora il giudice se n'accorse. Fecelne inteso, ma non valse: che perché l'avea detto tre volte convenne che fosse impeso. ${ }^{20}$

Analogamente, i burchi di color veneto ${ }^{21}$ in Inf. XVII I9-20 ("Come talvolta stanno a riva i burchi, / che parte sono in acqua e parte in terra») ricevono da Guido da Pisa un commento interessante per questi appunti: "sicut enim in partibus Lombardie naves, quas ipsi vocant 'burcos', stant aliquando medie in ripis et medie in aquis». ${ }^{22}$

Fra i barattieri dell'Inferno Ciampòlo navarrese si offre di far emergere dannati «Toschi o Lombardi» (Inf. xxII 99), per mostrarli a Virgilio e a Dante. A questa distinzione etnicoterritoriale corrisponde nella Divina Commedia un'identificazione anche linguistica, sicché dalla parlata di Dante Farinata riconosce in lui un Tosco (Inf. x 22) e il conte Ugolino un

${ }^{20}$ Il Novellino, a cura di Alberto Conte, Presentazione di Cesare Segre, Roma, Salerno Editrice, 200 I, p. I43. L'edizione stampa ollaro la seconda delle tre occorrenze di olaro, ma è evidente che, se pure la tradizione manoscritta toscana ha corretto la parola, su un olaroluno laro si regge il senso dell'equivoco (e cfr. ole), sicché la correzione è indispensabile.

${ }^{21}$ Cfr. Giovanni Nencioni, Il contributo dell'esilio alla lingua di Dante [1989], in Saggi e memorie, Pisa, Scuola Normale Superiore, 2000, pp. 3-2 I, a p. 7 .

${ }^{22}$ Guido da Pisa, Expositiones et Glose. Declaratio super 'Comediam' Dantis, a cura di Michele Rinaldi, voll. 2, Roma, Salerno Editrice, 20I3, vol. I, p. 56I. 
fiorentino (Inf. XXXIII I I ricordo incidentalmente che questo e altri luoghi sono continuamente citati e interpretati variamente durante le discussioni fiorentino-toscano-italiane sulla questione della lingua cinquecentesca). D'altra parte, entro un orizzonte italiano dai significati molteplici, la transizione dal natio loco (Inf. XIV I) alla regione (in senso lato) e all'Italia è continua: Virgilio, figlio di "parenti... lombardi» (Inf. I 68) e lombardo lui stesso, in lombardo ha congedato il greco Ulisse, come si apprende da Guido da Montefeltro che ne riporta le parole: "O tu a cu' io drizzo / la voce e che parlavi mo' lombardo, / dicendo "Istra ten va, più non t'adizzo" (Inf. XXVII I9-2I); e però nell' incontro con Sordello il passaggio da Mantova all'Italia è spontaneo, immediato: "e 'l dolce duca incominciava / "Mantua...", e l'ombra, tutta in sé romita, / surse ver' lui del loco ove pria stava, / dicendo: «O Mantoano, io son Sordello / de la tua terra!»; e l'un l'altro abbracciava. / Ahi serva Italia...» (Purg. vi 7I-76). ${ }^{23}$

Ricordo infine l'espressione, ricorrente nel De vulgari eloquentia, "vulgare latium» o «latium vulgare» (I x, 3, e più volte in seguito), ${ }^{24}$ che è volgarizzata nel finale del Teseida, dove Boccaccio così si rivolge alla sua opera: «ma tu, o libro, primo a lor [le Muse] cantare / di Marte fai gli affanni sostenuti, / nel volgar lazio più mai non veduti» (XII 84, 6-8) $)^{25}$. Il

${ }^{23}$ In questo paragrafo tocco brevemente questo e altri episodi, danteschi e no, esaminati in modo più dettagliato nel mio Fra "Lombardi", "Tusci" $e$ "Apuli": osservazioni sulle aree linguistico-culturali [1990], in Testi e chierici del medioevo, Genova, Marietti, I99I, pp. I I-4I, di cui è un'integrazione "Istra": una falsa ricostruzione dantesca?, in Omaggio a Gianfranco Folena, Padova, Editoriale Programma, I993, vol. I, pp. 4I9-28: qui aggiungo nuove testimonianze a ciò che ho raccolto in quelle sedi.

${ }^{24}$ De vulgari eloquentia, I x, 3, con il commento ad l. e i rinvii di Mirko Tavoni, in Dante Alighieri, Opere, Edizione diretta da Marco Santagata, Milano, Mondadori, 20 I I, vol I, pp. I240-4I.

${ }^{25}$ Teseida delle nozze d'Emilia, a cura di Alberto Limentani, in Giovanni 
volgar lazio di Boccaccio è però risemantizzato, perché equivale al volgar fiorentino, se è vero che, dieci e più anni prima di definire con modestia polemica il Decameron come composto da novellette scritte «non solamente in fiorentin volgare $\mathrm{e}$ in prosa $[\ldots]$ e senza titolo, ma ancora in istilo umilissimo e rimesso quanto il più si possono» (Iv Introduzione 3 ), ${ }^{26} \mathrm{a} \mathrm{Na}$ poli aveva dichiarato che il Filostrato (anteriore al Teseida) era scritto «in leggier rima e nel mio fiorentino idioma, con stilo assai pietoso" ( $\$ 29) .{ }^{27}$ Dunque in Boccaccio il significato di volgar lazio si allontana molto dal De vulgari eloquentia e dalla svalutazione delle quattordici varietà idiomatiche della penisola, senza alcuno sconto per il fiorentino, che Dante vi conduce. $^{28}$

4. Nel I5 I9 i Suppositi dell'Ariosto furono recitati con successo a Roma. Nel I 520 papa Leone x gli chiese un'altra commedia, e Ariosto revisionò rapidamente Il Negromante, che era rimasto a lungo nel cassetto. Il prologo scritto in occasione della prevista rappresentazione romana (che poi non

Boccaccio, Tutte le opere, a cura di Vittore Branca, Milano, Mondadori, I 964, vol. II, p. 66I.

${ }^{26}$ Decameron, a c. di Vittore Branca, Torino, Einaudi, I99 I ${ }^{6}$, pp. 459-60.

${ }^{27}$ Filostrato, a cura di Vittore Branca, in G. Boccaccio, Tutte le opere, cit., vol. II, p. 22.

${ }^{28}$ Una lucida intelligenza di latino nel senso di 'italiano' in Dante (e anche in Petrarca) è mostrata dal Trissino nel Castellano, dove pure è citato il luogo del Teseida sul volgar lazio (Scritti linguistici, a cura di Alberto Castelvecchi, Roma, Salerno Editrice, I986, pp. 69-72). Ottimo traduttore del De vulgari eloquentia, Trissino rende costantemente con volgare italiano il vulgare latium dantesco (si vedano i luoghi corrispondenti nella riedizione del suo volgarizzamento De la volgare eloquenzia, a cura di Francesco Montuori, in Dante Alighieri, Le Opere, edizione diretta da Enrico Malato, vol. III, De vulgari eloquentia, a c. di Enrico Fenzi, con la collaborazione di Luciano Formisano e Francesco Montuori, Roma, Salerno Editrice, 20 I 2). 
ebbe luogo) recita che la scena di Cremona, città in cui è ambientata la commedia, sia stata trasportata per arte negromantica a Roma, attraversando e assorbendo qualche parola lungo il viaggio: dallo Studio universitario bolognese e poi, attraverso Firenze e Siena, voci toscane; e tuttavia «in sì brieve termine / tanto appreso non ha, che la pronunzia / lombarda possa totalmente ascondere». ${ }^{29}$ Quattro anni dopo la prima edizione del poema, Ariosto poteva dunque ammettere, presentandosi a un pubblico non ferrarese, un colore lombardo che, lavorando a lungo sull'edizione definitiva dell'Orlando Furioso, non avrebbe poi dichiarato grazie alla guida linguistica del Bembo, al quale è dedicato un famoso omaggio nell'ultimo canto del poema. ${ }^{30}$

29 Tutte le opere, vol. Iv, Commedie, a cura di Angela Casella, Gabriella Ronchi, Elena Varasi, Milano, Mondadori, I974, p. 360 (vv. 52-54).

${ }^{30}$ Machiavelli, ammiratore dell'Orlando Furioso, nel Discorso intorno alla nostra lingua critica l'inadeguatezza dei motti nei Suppositi e, con un'incisiva contrapposizione fiorentino-lombarda, conclude il suo scritto in polemica contro i fiorentini persuasi delle tesi trissiniane e perciò «sì poco conoscitori de' beneficii ch'egl'hanno havuti da la nostra patria, che e' vogliono accomunare con essa lei nella lingua Milano, Vinegia, Romagna, et tutte le bestemmie di Lombardia» (ed. a c. di P. Trovato, Padova, Antenore, I982, p. 70). Dionisotti ha messo in rapporto la punta finale del Discorso machiavelliano e l'edizione, stampata a Firenze nel I 520 (all'incirca nello stesso tempo di composizione del Discorso) da Bernardo Giunta, dell'opera in terzine sull'arte della guerra del piacentino Antonio Cornazano: un'opera presentabile a un pubblico fiorentino solo a patto di essere - scrive Bernardo Giunta - liberata, compatibilmente con i vincoli imposti dalla rima e con la sua nativa e perciò inestirpabile barbarie, dai peccati gravissimi della lingua, dai vocaboli barbari, dagli errori grammaticali e dalle durezze retoriche, e insomma dalla lombarda barbarie (Machiavellerie, Torino, Einaudi, I980, pp. 352-363; a pp. 360-36I la prefazione di Bernardo Giunta). 
Tralasciando altri episodi, ${ }^{31}$ veniamo ai Ricordi del milanese Sabba Castiglione (circa I480-I 554), Cavaliere di Malta che, dopo aver prestato servizio a Rodi (I 505 - I 508), passò a impegni curiali in Roma; ricevuta nel i 5 i 5 la Commenda di Faenza, vi si ritirò progressivamente in una vita di studio o, petrarchescamente, di ozio religioso, concreta manifestazione di un cattolicesimo fedele agli studi umanistici e alle opere di pietà, e vissuto intensamente sul piano personale non meno che della difesa dell'ortodossia. ${ }^{32}$ A Faenza, dove trascorse il resto della vita, Sabba fondò infatti una scuola per bambini poveri e formò una biblioteca a utilità degli studiosi. ${ }^{33}$

Rispetto alla prima edizione dei Ricordi (I 546), la seconda, del I 549, è molto ampliata; leggeri incrementi sono nella terza (I 554), che è postuma. Le due edizioni in vita furono controllate, o almeno approvate, dall'autore, diversamente dalla terza. Quest'ultima, pur conservando la marcata impronta lombarda della precedente, mostra i segni di una continuativa

${ }^{31}$ Come uno spunto, segnalato nel mio Fra "Lombardi", "Tusci" e "Apuli", cit., p. 2 I9, dall' Orlandino del Folengo; sul quale indicazioni aggiuntive si ricavano da Mario Pozzi, Teofilo Folengo e le resistenze alla toscanizzazione letteraria [1978], nel volume Lingua, cultura, società, Alessandria, Edizioni dell'Orso, I989, pp. I37-55, alle pp. I48-50; Gianfranco Folena, Il linguaggio del "Caos" [ I 979], nel volume Il linguaggio del caos, Torino, Bollati Boringhieri, I991, pp. I47-I68, alle pp. I 53, I 59-60; Mario Chiesa, Don Teofilo Folengo "parteggiano" [2007], ora nel suo volume Saggi folenghiani, a cura di Giovanni Bàrberi Squarotti, Paolo Luparia, Mariarosa Masoero, Patrizia Pellizzari, Alessandria, Edizioni dell'Orso, 20I3, pp. 97-I I I, a pp. 99-IO2.

${ }^{32}$ Su Sabba è fondamentale Claudio Scarpati, Ricerche su Sabba Castiglione, nei suoi Studi sul Cinquecento italiano, Milano, Vita e Pensiero, I985, pp. 27-I 25; da vedere inoltre Sabba da Castiglione I480-I 554. Dalle corti rinascimentali alla Commenda di Faenza (Atti del Convegno, Faenza, I920 maggio 2000), a cura di Anna Rosa Gentilini, Firenze, Olschki, 2004.

${ }^{33}$ Scarpati, Ricerche, cit., p. 46. 
revisione linguistica in senso deregionalizzante, sicché è preferibile citare dal testo del I 549. ${ }^{34}$

Dall'opera, ricca di risentite riflessioni morali e satiriche, di critiche a quegli ambienti di corte che Sabba aveva abbandonato in età adulta rinunciando a una carriera che si annunciava promettente, ci limitiamo qui a estrarre ciò che è rilevante per il nostro argomento. Alla fine del libro, Sabba compone un'Epistola delle lingue d'Italia (tale è il titolo che figura in un testimone manoscritto) ${ }^{35}$ diretta a un Venerabile in Christo patre (di identificazione incerta, come si dirà in seguito), lettore dei Ricordi prima della stampa. Ne ha ricevuto un giudizio favorevole, ma con una riserva sulla lingua, ritenuta lontana dal toscano. Dell'argomento linguistico Sabba ha già parlato più volte a voce con l'interlocutore, e ora gli manda una replica articolata:

anchora ch'io conoscessi la lingua thosca essere la più dilicata, dilettevole, dolce, ornata, \& copiosa, che nessuna altra d'Italia, pure essendo io Italiano, mi è parso nelle mie poche compositioni usare sempre la mia Italiana lingua, \& massimamente la Lombarda, per essere io Lombardo, anzi pur Lombardozzo, come dice il thosco (c. CIX). ${ }^{36}$

Sabba cita il titolo delle ccnto novelle antiche (il Novellino), e sa che prima dei trecentisti correvano in volgare parole poi uscite dall'uso, mostrando di aver chiara la distinzione, certo appresa da Bembo, tra la lingua dei trecentisti e quella dei loro

\footnotetext{
34 Sulle edizioni a stampa e i testimoni manoscritti, cfr. Scarpati, Ricerche, cit., pp. 83-9I; sul colorito linguistico delle tre edizioni e la fortuna cinquecentesca dell'opera, Paolo Trovato, La lingua di Sabba, in Sabba da Castiglione I480-I 554, cit., pp. 73-93.

${ }^{35}$ Scarpati, Ricerche, cit., p. 87, e p. I I 4.

${ }^{36}$ Cito qui e in seguito dall'edizione del I 549, avvertendo che l'Epistola, si legge, con alcune varianti manoscritte, anche in Scarpati, Ricerche, pp. I 22-25.
} 


\section{predecessori duecenteschi. ${ }^{37}$ Secondo il suo giudizio le opere}

${ }^{37}$ Ricordi, ed. cit., c. cxx. Nelle Prose della volgar lingua uno dei significati (precisati dal contesto) di antico, anticamente denota una fase di lingua che precede l'età esemplare, dunque il tempo di Petrarca e Boccaccio. Nelle Prose le citazioni dal Novellino sono accompagnate costantemente dalla qualificazione dell'antichità linguistica, perciò da evitare (cfr. il commento di Carlo Dionisotti a Pietro Bembo, Prose e rime, Torino, Utet, I 960: III XVI, p. 2I0; XXV, p. 227; LI (bis), p. 265; LXVII, p. 289; LXVIII, p. 29I; LXX, p. 294). Il Novellino, tuttavia, non è mai indicato specificamente, sicché la nota dell'antichità poteva essere ricavata da Sabba più facilmente dall'edizione a stampa del testo: Le ciento novelle antike, Bologna, Benedetti, I 525, che quell'antichità (in senso assoluto, e relativo al Decameron) annunciava fin dal titolo. Nella dedica a Goro Gheri, Carlo Gualteruzzi (che pubblicava l'opera su diretta ispirazione del Bembo) ne scriveva: "di tutte le cose in prosa volgare scritte, che insino a questo dì sono alla mia notitia pervenute, giudico essere la più antica...». Le Ciento novelle antike (dove già antike del titolo esprime l'arcaismo ortografico) escono nel I 525, lo stesso anno delle Prose del Bembo; Sabba riferisce di aver incontrato Bembo a Padova nel I 527, sicché poté ben conoscere le fresche novità delle Ciento novelle e delle Prose (per l'incontro con Bembo cfr. Scarpati, Ricerche, cit., p. 45 e p. I I7). Che poi Bembo si sia detto d'accordo sulle idee linguistiche di Sabba è cosa che si può spiegare in vari modi (Sabba asserisce di essere stato approvato anche da Sannazaro, da lui incontrato a Napoli, al suo ritorno da Rodi, nel I 508, dunque in un'epoca di cultura linguistica molto lontana da quella di vent'anni dopo). Se poi è consentita un'osservazione che va oltre i limiti cronologici primocinquecenteschi, sulla scia delle Ciento novelle antike va considerata la seconda edizione a stampa del Novellino, e cioè il Libro di novelle, et di bel parlar gentile, Firenze, Giunti, I 572. Nella premessa, firmata da Filippo e Iacopo Giunti, in realtà di mano di Vincenzo Borghini, si fanno molte lodi al Bembo, «a cui tutte le buone lettere, ma particularmente la nostra Città et la nostra favella infinitamente debbe», indicato come l'ispiratore dell'edizione Gualteruzzi del I 525 . Nell'edizione del I 572 si legge ancora che Bembo non solo raccomandò la buona lingua, ma «non tacque delle [voci] più antiche et già tralasciate», fondandosi tra l'altro sulle cento novelle duecentesche; delle quali, tuttavia, Borghini corregge l'ortografia arcaica dell'edizione del I 525 (tralascio naturalmente le profonde differenze testuali intercorrenti tra le due edizioni, e rimando direttamente alla citata edizione del Novellino a cura di Alberto Conte). Logico corollario 
delle Tre Corone sono non solo di lingua toscana ma anche italiana; segnala l'entrata recente in italiano di francesismi e spagnolismi (che non guarda con favore, perché gli è chiaro che queste innovazioni linguistiche, di fronte alle quali Baldassar Castiglione si era mostrato favorevole nel Cortegiano I xxxIv ${ }^{38}$ sono l'effetto del pessimo stato politico dell'Italia dei suoi anni: Ricordi, cc. xxxvı $v$-xxxvII). L'idea dell'italianità (e non della pura toscanità) delle Tre Corone autorizza l'ipotesi di una conoscenza dell' italianismo linguistico del Trissino da parte di Sabba ${ }^{39}$ (che però non si riflette sul suo registro linguistico, come sono sufficienti a mostrare le citazioni già fatte e le successive). A Trissino accenna quest'affermazione, nella stessa Epistola al Venerabile in Christo patre:

Et però io mi risolvo, come ho di sopra detto, che sì come sono Italiano, così intendo di usare come sempre ho usato ne le mie inettie la Italiana lingua, con valerme de vocaboli di qual si voglia Provincia di essa (c. CXx).

Mi pare, tuttavia, che la posizione teorica di Sabba sia un po' oscillante, tra quest'ultima citazione di marca trissiniana ortodossa e la bandiera lombarda (se non lombardozza) dalla quale si ricava un orizzonte linguistico di tipo italiano con una netta propensione settentrionale: rispecchia, indipendentemente dalla lieve oscillazione teorica che sembra di cogliere, la situazione effettiva della prassi scrittoria di Sabba. Questi prosegue

dell'arcaicità linguistica del Novellino è, nell'edizione fiorentina del I 572, un glossario di otto pagine: "Dichiarationi d'alcune voci antiche, che si trovano per entro il libro». $\grave{E}$, con ogni evidenza, un interesse linguistico che avrà il suo esito nel dizionario della Crusca del I6I 2.

${ }^{38}$ Il libro del Cortegiano, a cura di Bruno Maier, Torino, Utet, I964², pp, I $4 \mathrm{I}-42$.

39 Secondo Scarpati, invece, Sabba non conoscerebbe Trissino (Ricerche, p. II9). 
dichiarando di scegliere «vocaboli buoni, usitati, aprovati, \& usati da gli Auttori autentici volgari», e di evitare gli «inusitati, plebei, \& contadineschi, i quali sanno di rancio»: parole che contengono lo spunto già ricordato sugli arcaismi del Novellino (c. CXX).

Dall'Epistola posta a conclusione dei Ricordi si può utilmente risalire a due testi di dedica: il primo Al pio et candido lettore; l'altro è un Proemio al nipote Bartolomeo Castiglione, anche lui Cavaliere di Malta, subentrato nel I 544 a Sabba nella Commenda di Faenza. Nel Proemio Sabba parla del problema linguistico, e definisce la sua prosa in termini negativi, perché vi manca «quel ornamento, \& quella elegantia del parlar toscano, o corteggiano quale hoggi universalmente se usa per la Italia» (c. vII): ribadisce dunque lo scostamento dal toscano, rimproveratogli dal venerabile in Christo patre, e si dice anche estraneo al parlar ... corteggiano invalso in Italia. La qualifica di corteggiano rinvia ovviamente alla Curia romana, frequentata molti anni prima da Sabba, e insieme (lo prova l'universale diffusione in Italia di quella lingua), il modo di parlare e di scrivere corrente nelle Corti signorili. Come è evidente, Sabba rinvia ai modi espressivi dei ceti dirigenti, dei quali ha scelto di non far parte e nei quali non s'identifica; e una valutazione analoga si estende, almeno per chi, come lui, non è toscano, alla varietà fiorentino-toscana.

Dalla prosa di Sabba, oltre che da queste insistite dichiarazioni, risulta la pratica di una lingua aliena da una toscanità artificiosa, perché non nativa, e da ornamenti retorici che, fuori di Toscana, contrassegnano l'espressione degli ambienti elevati. In termini positivi Sabba ricerca un volgare efficace, lontano dal dialetto e però stretto a un'espressione marcatamente lombarda e cioè settentrionale. Sabba ammette senza difficoltà il prestigio del toscano, cosa che non gl'impedisce di scrivere, rivolgendosi nel Proemio al nipote (e anzi, non fiorentinamente, nepote): 
essendo io lombardo, \& scrivendo a voi qual parimente sete lombardo, accioché meglio fosse inteso me parve a dovere scrivere in lingua lombarda la quale, anchora che non habbia quella leggiadria delicatezza, \& coppia che la toschana, pur quando io habbia con essa espresso li mei concetti della mente me contentarò haver usato el mio proprio idioma, quale ello se sia (c. viv.).

Il registro di Sabba non coincide con una scelta di assoluta dialettalità, non coincide cioè con la posizione estremistica difesa (solo teoricamente) da Pomponazzi nel Dialogo delle lingue di Sperone Speroni (scritto probabilmente all'inizio degli anni Trenta e pubblicato nel I 542): il filosofo mantovano polemizza aspramente con gli studia humanitatis che puntano sulla padronanza del latino classico e del greco dei filosofi antichi (di fatto di Aristotele e dei suoi commentatori, oggetto di studio nelle università), e non risparmia il nascente classicismo del volgare (di cui era corifeo il Bembo, uno dei personaggi del Dialogo speroniano). Secondo Pomponazzi sarebbe meglio non perder tempo nello studio dei verba e di forme linguistiche prive di contenuto, per impegnarsi direttamente nelle res, e cioè nella filosofia, lasciando a ognuno la facoltà di esprimersi nell'idioma materno. Tuttavia, tranne qualche battuta che si concedeva a lezione nel suo volgare settentrionale, i manoscritti dei corsi di Pomponazzi, e i suoi libri a stampa, sono nel disadorno ma tecnico ed efficiente latino della scolastica: era evidente che filosofare nei diversi idiomi locali avrebbe complicato il problema, per non dire della Babele linguistica in cui sarebbero precipitati gli Studi di Padova o di Bologna, frequentati da una popolazione italiana e internazionale, con una presenza importante di scolari di lingua germanica e slava, oltre che romanza.

La soluzione dialettale, accessibile al teatro di un Ruzante (cresciuto, peraltro, nell'ambiente dello Speroni), era impraticabile tanto per l'insegnamento universitario della filosofia quanto per la prosa dei Ricordi di Sabba; e però, lasciando da 
parte il latino scolastico e gli idiomi locali, e tornando al volgare, è anche chiaro che, nella media delle stampe settentrionali in volgare del Cinquecento maturo, la marcatura locale di Sabba è particolarmente intensa. È effettiva, dunque, e non solo enunciata, la tenuta di un registro linguistico al di sotto della lingua cortigiana e del toscano, benché gli argomenti morali, sociali, religiosi siano di forte impegno culturale, non riguardino cioè il mondo delle arti e dei mestieri e insomma delle cose, nel quale lo scrittore non toscano attinge più facilmente a un patrimonio lessicale regionale.

Una riprova si ottiene facilmente paragonando Sabba con il suo parente Baldassar Castiglione. Nella dedica del Cortegiano Castiglione insiste sulla differenza fra toscano e lombardo in fatto di latinismi: il toscano modifica l'origine latina, il lombardo la conserva, argomento favorevole a questo contro quello. Soprattutto, Castiglione rivendica, in modo non troppo dissimile da Sabba, il proprio abito linguistico:

né credo che mi si debba imputare per errore lo aver eletto di farmi più tosto conoscere per lombardo parlando lombardo, che per non toscano parlando troppo toscano; per non fare come Teofrasto, il qual, per parlare troppo ateniese, fu da una simplice vecchiarella conosciuto per non ateniese ${ }^{40}$.

La distanza dal toscano è in Baldassarre molto minore che in Sabba; non per nulla questi rifiuta la categoria della lingua cortigiana, mentre l'opera di Baldassarre è una costruzione integrale della cortigianìa, inclusa la lingua. ${ }^{41}$

${ }^{40}$ Il libro del Cortegiano, ed. cit., p. 75. Nelle sue Ricerche più volte citate Scarpati tende ad avvicinare eccessivamente Sabba Castiglione e Baldassar Castiglione.

${ }^{41}$ Diversamente interpreta Trovato, secondo il quale solo per un topos $\mathrm{di}$ modestia Sabba si definirebbe «al di sotto del parlare toscano e cortigiano»; e poco dopo lo stesso afferma che quella di Sabba sarebbe «una scelta di campo argomentata e inequivocabile: antifiorentina, ma interna e non al 
Tornando ora a Sabba, la dedica al Al pio et candido lettore, di cui non si è ancora detto, contiene un'informazione di grande interesse. In una dichiarazione antiretorica, da non prendere alla lettera, l'autore dice di avere scritto «nella sua volgar lingua, o veramente materna» (c. v), perché il suo pubblico non è formato dai dotti che conoscono il latino o il greco o che imitano Dante Petrarca Boccaccio, è composto insomma da lettori che sono alfabetizzati, ma che non intendono un volgare letterario, non conoscono il latino, necessario per capire la ricercatezza del toscano letterario e della lingua cortigiana o toscaneggiante. Ecco perché Sabba rifiuta le categorie dell'affettazione toscana o delle eleganze cortigiane, e, aggiungiamo ora, si sceglie come guida il «Reverendo Padre Frate Leandro delli Alberti da Bologna» (c. vv). A lui, domenicano e inquisitore a Bologna, Sabba si rivolge non solo come a sicuro custode dell'ortodossia, ma come a un alleato anche nell'idea di un volgare distinto da quello praticato dalla cultura elevata, e con il tono di chi sa che l'interlocutore gli darà il suo consenso. Questo nesso è sfuggito finora, perché gli interpreti hanno pensato al rapporto di Sabba con Alberti per il ruolo di quest'ultimo nel contrastare l'eresia luterana e per l'importantissima Descrizione di tutta Italia, uscita nel I 550: un'opera formalmente, e consapevolmente, volutamente dimessa che, per inciso, ebbe grande fortuna di ristampe nella seconda metà del Xvi secolo, lo stesso arco di tempo nel quale anche i Ricordi di Sabba conoscono un forte successo. Non è però nella Descrizione, ma in altre scritture, meno note, di Alberti, che va cercato il nesso con le idee controcorrente di Sabba. Nel I 54I Alberti pubblicò le Historie di Bologna, facendole precedere da alcune dichiarazioni sulla strategia espressiva dell'opera:

di sotto della lingua letteraria» (La lingua di Sabba, cit., pp. 84-85). 
Forse parerà alli sollevati ingegni non essere questa nostra opera per il loro gusto, etiandio dilettandosi della volgar lingua, per non essere scritta con quella polita e dolata lingua Thosca, come hora molti scriveno, non havendo seguito Dante il Boccaccio et il Petrarcha con le osservationi del Fortunio, Bembo, Claritio [Girolamo Claricio], Philoteo [Achillini], Tressino, e de molti altri, curiosi, radi e nobili scrittori di nostra età, della volgare lingua ristoratori, ma se consideraranno la cagione perché così l'ho scritta senza quelle osservationi m'haveranno iscusato. Ho pigliato questo incarico non curandomi di tanta osservanza, per beneficio del volgo, e di quelli non hanno intelligenza de latine lettere, né anchora son'occupati nelle osservationi della lingua Thosca, conciosia che quelli hanno peritia de latinità, potranno sodisfare a suoi honesti voti leggendo l'historie del facondo Bocchio [Achille Bocchi], et non manco gli osservatori della lingua Thosca, havendo cognitione de latini et osservati vocaboli del Petrarcha, di Dante et del Boccaccio, per li quali facilmente intendere si possono le cose latine, benché dal latino discosto sovente paiano. E così solamente ho atteso a descrivere la sostanza delle cose con vera integrità, non molto curandomi degli accidenti. Perché s'havess'io voluto ornare questa nostra opera, con quelli esquisiti vocaboli da molti eccellenti scrittori della volgare lingua hora usati, non essendo poi dal rozzo volgo inteso overo da pochi, sovente passarebbeno molti di mala voglia parte dell'historia senza intelligenza, e perciò serebbe senza frutto e senza piacere ${ }^{42}$.

\section{A ritroso nel tempo, è assai chiarificatrice la premessa a un testo agiografico volgarizzato, rimaneggiato, semplificato dall'Alberti, la Vita della Beata Colomba da Rieti ( I 52 I):}

\footnotetext{
${ }^{42}$ Libro primo della Deca prima delle Historie di Bologna, Bologna, Bartolomeo Bonardo e Marco Antonio Grosso, I 54I. Tra gli autori citati dall'Alberti figura l'Achillini, per le Annotationi della volgar lingua di Gio. Philoteo Achillino, M.D.xxxvi (nel colofone: In Bologna per Vicenzo Bonardo da Parma et Marcantonio da Carpo), un dialogo di cui è interlocutore l'Alberti: gli è attribuita una posizione fiorentino-letteraria di marca bembesca (contro la quale polemizza Achillini, sostenitore di un volgare cortigiano di colore prevalentemente bolognese: si veda M. Vitale, Dottrina e lingua di G. F. Achillini teorico della lingua cortigiana [1987], in Studi di storia della lingua italiana, Milano, LED, I992, pp. I I I-26), smentita dalla teoria e dalla prassi dell'Alberti.
} 
essendo ne' tempi nostri apparuta quella lampeggiante e vaga stella, e singolare imagine e specchio de vertuti, cioè la inclita vergine de Christo suore [sic] Colomba Reatina, (la quale ha con sue splendenti opre illuminato li mortali) acciò che tanto lume fosse profittevole a qualunche grado de persone, e non solamente alli Umbri, Etrusci [Toscani], e litterati, ma anchor a tutti Italiani, et massimamente a quelli non hanno peritia nelle latine lettere, ho.lli eretto e driciato questa celeberrima statoa, e cotesto eminente e soperbo arco triomphale, non di candido marmo, né di altra pietra, né ancho di duro metallo, ma di perpetue lettere. Conciosia che essendo gionti a mie mani, un libro iscritto in latino, composto dal Venerando padre Frate Sebastiano Perugino egregio dottore di sacra Theologia, huomo anticho, e di non puoca riputatione, che contiene la Vita di essa saggia sposa di Christo, [...] ho pigliato cotesta faticha [...] di tradurlo in volgare, et ancho breviarlo. ${ }^{43}$

La stessa mira a un pubblico ignaro di latino, per il quale le eleganze del volgare letterario modellato almeno in parte sul latino risultano incomprensibili, è alla base del criterio di Sabba, interessato a una prosa accessibile, e però ricca di cultura intellettuale e religiosa. Tralasciando il fatto che nella prima edizione i Ricordi si fregiano di una lettera in latino di caldo consenso per i contenuti morali e religiosi firmata proprio da Leandro Alberti, ${ }^{44}$ è sul terreno del volgare che le prose di Alberti e di Sabba, diverse per colorito linguistico, s'incontrano sui principi di fondo. Anche Sabba, infatti, si dirige a un pubblico inesperto di latino (che è poi quello maggioritario rispetto all'élite colta), e generalizzando dal nipote dedicatario dell'opera al pubblico dei lettori dichiara di

\footnotetext{
${ }^{43}$ Vita della Beata Colomba da Rieti dil Terzo ordine di S. Domenego Sepolta a Perugia, Bologna, Girolamo delli Benedetti, I 52 I. Notizie più circostanziate nel mio lavoro Edizioni di testi e storiografia: a proposito di due riedizioni parziali dell' "Italia illustrata» di Biondo Flavio e della "Descrittione di tutta Italia» di Leandro Alberti, "Giornale Storico della Letteratura Italiana», I 84 (2007), pp. 399-422.

${ }^{44}$ È riportata da Scarpati, Ricerche, cit., p. 84.
} 
scrivere "ad un giovene e cavaglieri e ad altre persone ch'hanno il natural desio di sapere, ma non sanno il latino; li quali senza comparazione sono assai più di quei che 'l sanno». ${ }^{45}$ Dopo aver preso le distanze da «questi gran professori delli nostri tempi della volgar lingua italiana», e da Dante, Petrarca, Boccaccio, oggetto del loro studio, dichiara di scegliere come guida il suo «reverendo padre frate Leandro delli Alberti da Bologna de l'ordine de' predicatori». Perciò non credo che con Leandro Alberti si possa identificare il venerabile Patre destinatario dell'Epistola $;^{46}$ con ogni cautela, avanzo l'ipotesi di un interlocutore polemico sulla posizione lombarda di Sabba (Alberti non avrebbe potuto chiedergli un più alto quoziente letterario), da cercare piuttosto in area toscana.

Come è noto, il volgare toscano o cortigiano è la linea da cui sorge l'italiano letterario del Rinascimento. Questo volgare è troppo difficile per il grosso del popolo cristiano, femminile non meno che maschile, di cultura modesta ma desideroso di leggere, ed è per questo pubblico che Alberti e Sabba individuano uno spazio linguistico volgare ben distinto da quello dei letterati e offrono i loro libri di salda base religiosa, e insieme veicolo di idee umanistiche trasmesse in una prosa semplice. Mentre il volgare letterario di Bembo, Ariosto, Castiglione raggiunge le altezze del latino umanistico, Leandro Alberti e Sabba Castiglione praticano una via umile e però abbondante di contenuti morali, e reinterpretano quell'esigenza comunicativa - l'altra faccia dello stretto rapporto con il latino - che

${ }^{45}$ Cito da Scarpati, Ricerche, cit., p. 93.

${ }^{46}$ Così suggerisce Scarpati, Ricerche, cit., pp. 72-73, I I 4-I I 5, che come espressione delle idee linguistiche dell'Alberti usa la testimonianza non molto attendibile di Achillini, nelle cui Annotationi la posizione toscanista dell'Alberti (affermata alle cc. 8; I 3v) è minoritaria, e cede alla rivalutazione del bolognese, sicché non escluderei che ad Alberti tocchi il ruolo di chi sostiene una tesi inaccettabile, con scarsa fedeltà al pensiero effettivo del personaggio (e cfr. la n. 4I). 
è coessenziale alla vita della Chiesa. Non saprei dire quanto questa via, parallela alla lingua colta, abbia avuto consistenza; se si fosse consolidata così come quella della grande letteratura cinquecentesca, la frattura tra l'aristocrazia e il popolo sarebbe stata assai meno profonda di quanto non fu.

5. In una novella del Bandello Zanina, moglie di ser Gandino bergamasco (uno dei tanti mariti gelosi della novellistica, destinati a esser traditi dalle mogli), si chiude nella sua camera per leggere opere della letteratura in volgare della quale fa, come la vedova del Corbaccio, un uso pornografico, mescolando con "la Nanna o sia Raffaella de l'Aretino" ${ }^{47}$ due delle Tre Corone e l'Ariosto: «il Petrarca, le Centonovelle o il Furioso». Prono ai capricci della moglie, «ser Gandino, a ciò che la moglie troppo leggendo non s'affaticasse, faceva egli il lettore, e con quella sua goffa pronunzia bergamasca le leggeva tutto ciò che ella comandava». ${ }^{48}$

È noto lo scarso prestigio del bergamasco, dialetto spesso portato sulle scene comiche del Cinquecento; e tuttavia il volgare letterario pronunciato e storpiato in modo marcatamente bergamasco da ser Gandino non è rappresentato (fatta la debita parte alla sprezzante connotazione comica incollata su quel dialetto) in modo sostanzialmente diverso dal dialetto di Milano (la città rimasta per sempre nel cuore del Bandello) in bocca alle sempre lodate donne milanesi:

a me, per dirne ciò ch'io ne sento, pare che niente manchi loro a farle del tutto compite, se non che la natura le ha negato uno idioma conveniente a la beltà, ai costumi e a le gentilezze loro. Ché in

\footnotetext{
${ }^{47}$ La Nanna è personaggio delle Sei giornate dell'Aretino, al quale per un errore di memoria Bandello attribuisce anche la Raffaella di Alessandro Piccolomini.

${ }^{48}$ Novella I xxxiv, in Matteo Bandello, Tutte le opere, a cura di Francesco Flora, Milano, Mondadori, I952 $2^{3}$, voll. 2 (I, p. 4I9).
} 
effetto il parlar milanese ha una certa pronunzia che mirabilmente gli orecchi degli stranieri offende. Tuttavia elle non mancano con l'industria al natural diffetto supplire, perciò che poche ce ne sono che non si sforzino con la lezione dei buon libri volgari e con il praticare con buoni parlatori farsi dotte, e limando la lingua apparare uno accomodato e piacevole linguaggio, il che molto più amabili le rende a chi pratica con loro. ${ }^{49}$

Non solo ai bergamaschi ma anche alle dame milanesi conviene migliorare la pronunzia leggendo libri volgari e conversando con buoni parlatori (maschi istruiti, evidentemente); si noti inoltre che il parlar milanese va inteso come dialetto, mentre ser Gandino piega a una forte prosodia bergamasca $\mathrm{i}$ testi in volgare letterario prediletti dalla moglie. Altrove si legge che quando elogia Milano, sua patria (ideale), Bandello trova dei contraddittori:

e se io domando loro per qual cagione non vogliono che io dica bene de la patria mia, altro insomma non mi sanno che rispondere se non che il parlar milanese è troppo più goffo che parlar che s'usi in Lombardia, e quasi che non si vergognano chiamarlo più brutto che il bergamasco. Ma io non trovo mai, - per l'ordinario, dico, - che i tedeschi parlino altro linguaggio che il loro, i francesi quello di Francia, e così ogni nazione il parlar suo nativo. Io non vo' già dire che la lingua cortegiana non sia più limata de la milanese, ché mi crederei dir la bugia; ma bene mi fo a credere che nessuna lingua pura che s'usi del modo ov'è nata, che sia buona. Si pigli pure e la toscana e la napoletana e la romana o qual altra si voglia, che tutte, non ne eccettuando alcuna, hanno bisogno d'esser purgate e diligentemente mondate, altrimenti tutte tengono un poco del rozzo ed offendono gli orecchi degli ascoltanti. ${ }^{50}$.

Dunque la lingua materna e locale (o parlar nativo) è inaccettabile, a Milano non meno che a Bergamo, con un giu-

\footnotetext{
${ }^{49}$ I IX, ed. cit., I, pp. I I 7-I 8.

${ }^{50}$ II xxxI, ed. cit., I, pp. 987-88.
} 
dizio, aggiungiamo, che vale per l'oralità e in modo ancora più netto per la scrittura. La natura del parlar nativo deve essere perfezionata dall'industria (altri nel XVI secolo avrebbe detto dall'arte), in modo da raggiungere un'espressione orale curata, consapevole, anche se ricca di contrassegni locali e regionali. Su un registro più alto è la lingua cortigiana, sapiente mescolanza mistilingue che riflette l'ambiente non provinciale delle corti; della conversazione cortigiana vogliono essere un'espressione le novelle di Bandello: che, come si sa, aderisce non già alla linea Alberti-Sabba da Castiglione ma a quella del Cortegiano di Baldassar Castiglione.

$\mathrm{E}$ in rapporto, anche se non immediato, con ciò che si è appena affermato la novella della fosca storia di Alboino e Rosmunda, che racconta la conquista longobarda e i primi tempi dei nuovi padroni in Italia. In apertura, prima della narrazione propriamente detta, lo scrittore menziona i marchesi Pallavicino, i Visconti, i Landriani, i Vicedomini, i Valvassori, i Cattanei e, fuori di Lombardia, i Malaspina, i Savorgnan, i Canossa, gli Este: tutte famiglie di origine longobarda (vera o presunta), il che autorizza la transizione alla novella, libero rifacimento della tradizione storica. ${ }^{51}$

Bandello era nato a Castelnuovo Scrivia, che nella geografia sua e del suo tempo faceva parte della Lombardia. Trovandosi nel paese natale l'autore, nella dedica della novella I XXIII (inviata significativamente al cugino Giacomo Francesco Bandello: ${ }^{52}$ c'è aria di famiglia), narra di una discussione sorta tra alcuni commensali sulla «fondazione de la patria nostra, essendoci chi voleva che l'origine sua da' goti venisse» e chi invece era convinto di un'origine longobarda. Interviene

\footnotetext{
${ }^{51}$ III XVIII, ed. cit., II, pp. 347-48.

52 Sui personaggi storici della novella cfr. Carlo Godi, Bandello. Narratori e dedicatari della prima parte delle "Novelle», Roma, Bulzoni, I996, pp. I 24-28.
} 
Matteo Bandello e persuade i presenti che Castelnuovo ebbe origine, nel tempo di Teodorico, da un insediamento di ostrogoti e di soldati romani. ${ }^{53}$ Segue una novella sulle origini di Castelnuovo, fatte risalire all'amore di un ostrogoto (manca nel racconto ogni traccia del gruppo etnico, quello dei soldati romani), Bandelchil figlio di Velamiro (si noti l'onomastica esotica) per la bella e nobile Aloinda figlia di Clisterdo. Grazie a un amico, Teialac, che fa da intermediario (secondo un topos narrativo di origine medievale), Bandelchil e Aloinda s'incontrano, si amano, si sposano, generano molti figli:

E temperandosi poi i vocaboli barbari con l'italiana pronunzia, i descendenti da Bandelchil si chiamarono Bandelli, come oggidì ancora sono chiamati. ${ }^{54}$

Oltre che l'origine ostrogota (e, in posizione poco marcata, anche romana) di Castelnuovo Scrivia la novella offre la mitica genealogia dei Bandello: si apprende infatti che, conservato fra gli «antichissimi scritti» posseduti da un Enrico Bandello, lo scrittore ha visto un "privilegio autentico d'Ottone, primo di questo nome imperadore», che dava ai Bandello una signoria nei pressi di Castelnuovo. Con il matrimonio di una "madonna Agnese Bandella» con un "messer Bernardo da la Torre», la famiglia seguì le sorti dei Della Torre, sconfitti dai Visconti ed esuli da Milano, sicché i Bandello rimasero privi «de la signoria de le lor terre, né mai quella ricuperarono»..$^{55}$ Né va dimenticato l'impagabile vezzo di arricchire l'album di famiglia con un «san Bandello goto» che avrebbe culto in Francia. ${ }^{56}$

Le vicende, sfortunate ma nobilitanti, dei Bandello clien-

\footnotetext{
${ }^{53}$ Ed. cit., I, p. 293.

${ }^{54}$ Ivi, p. 302.

${ }^{55}$ Ivi, p. 295.

${ }^{56}$ Ivi, p. 294.
} 
ti dei Torriani danno ragione del loro trasferimento a Milano, la città dove lo stesso Matteo si era formato. Dopo molte vicende dovute alle turbolenze militari e politiche di anni difficilissimi, tra le quali l'omicidio politico, organizzato da Alfonso D’Avalos, marchese del Vasto, di Cesare Fregoso (I 54I), uomo d'armi e informatore della Francia, Bandello, già segretario del Fregoso, restò fedele alla vedova Costanza Rangone (era stato lui a combinare il matrimonio), e passò l'ultima parte della vita in Francia. La vicenda dell'esilio in nome della fedeltà al proprio signore ripeteva dunque l'antico esilio, con ogni probabilità frutto d'invenzione, dei Bandello che avevano seguito nella mala sorte i Della Torre.

In questo clima acquistano maggior significato le note professioni bandelliane di lombardismo e di distanza da Firenze e dalla Toscana: dichiarazioni che importano per la lingua e lo stile e radicano la scelta della prosa in una mitizzazione familiare, etnica, territoriale alternativa alla romanità umanistica. ${ }^{57}$ Nella dedica Ai lettori, innanzi alla prima parte delle novelle:

Io non voglio dire come disse il gentile ed eloquentissimo Boccaccio, che queste mie novelle siano scritte in fiorentin volgare ${ }^{58}$, perché direi manifesta bugia, non essendo io né fiorentino né toscano, ma lombardo. E se bene io non ho stile, ché il confesso, mi sono assicurato a scriver esse novelle, dandomi a credere che l'istoria e cotesta sorte di novelle possa dilettare in qualunque lingua ella sia scritta. $^{59}$

\footnotetext{
57 Un po' diversamente intende A. Ch. Fiorato, Bandello entre l'histoire et l'écriture, Firenze, Olschki, I979, pp. 2-I3, da vedere comunque per la distinzione tra realtà storica e trasfigurazione mitizzante, in parte documentabile, nell'albero genealogico proposto dallo scrittore.

${ }^{58}$ Ripresa letterale (apocope compresa) dell'espressione usata nella difesa della IV giornata del Decameron $\left(\$_{3}\right)$.

${ }^{59}$ Ed. cit., I, p. 4.
} 


\section{E, dedicando la Prima parte a Ippolita Sforza Bentivoglio, dice di avere scritto le novelle per ispirazione della gentildonna:}

Se poi, come di leggiero forse avverrà, cose assai vi saranno rozze, mal esplicate, né con ordine conveniente poste, o con parlar barbaro espresse, a la debolezza del mio basso ingegno l'ascriva e al mio poco sapere, e pigli in grado il mio buon volere, pensando ch'io son lombardo e in Lombardia a le confini de la Liguria nato [...] e che, come io parlo così ho scritto. ${ }^{60}$

Naturalmente non si può credere a un parlare trasferito di peso nello scrivere, senza dire che il parlare di Bandello non era quello della pura lingua nativa, ma quello affinato, si può supporre, dall'esperienza cortigiana. Infine, nella dedica ai lettori della terza parte (si noti il giudizio di tipo trissiniano sulla lingua di Petrarca):

Ora ci saranno forse di quelli che [...] diranno ch'io non ho imitato i buoni scrittori toscani. A questi dirò io, come mi sovviene altrove d'aver scritto, che io non sono toscano, né bene intendo la proprietà di quella lingua, anzi mi confesso lombardo, anticamente disceso da quelli ostrogoti che, militando sotto Teodorico loro re ed avendo le stanze a Dertona, edificarono la mia patria ne la via Emilia tra i liguri cisapennini, non lungi da la foce de la Schirmia [...]. Essa colonia chiamarono Castelnuovo [...]. Non sarebbe adunque gran meraviglia se io talora usasse alcuna parola triviale, e poco usitata, che spirasse alquanto del gotico. Se la lingua tosca mi fosse stata natia o apparata l'avessi, molto volentieri usata l'averei, perciò che conosco quella esser molto castigata e bella. Nondimeno, per quello che a me ne paia, il coltissimo ed inimitabile messer Francesco Petrarca, che fu toscano, ne le sue rime volgari non si truova aver usate due o tre voci pure toscane, perché tutti i suoi poemi sono contesti di parole italiane, communi per lo più a tutte le nazioni de l'Italia ${ }^{61}$.

${ }^{60}$ Ed. cit., I, 8.

${ }^{61}$ Ed. cit., II, pp. 247-48. 
Dichiarazioni di lombardismo in chiave di distinzione dal toscano, o di opposizione, sono numerose: è stata segnalata ${ }^{62}$ una professione di lombardismo nei Paradossi (I 543) di Ortensio Lando, milanese, che nella dedica del secondo libro dice che avrebbe scritto volentieri in toscano,

ma ricordandomi d'esser nato nella città di Milano, et fra Longobardi longamente vissuto mi venne al cuore una certa diffidenza la quale di sorte m'impaurì che subito abandonai il pensiero di scrivere toscanamente, et ricorsi a quella forma di parlare che già preso havea, parte dalla mia nudrice, parte ancora da migliori scrittori ${ }^{63}$.

Senza insistere su altre testimonianze, mi limiterò a ricordare la presenza della dualità nella predicazione dal secondo Cinquecento alla metà del XviıI secolo. ${ }^{64}$

6. È ben nota la Rinunzia avanti nodaro degli autori del presente foglio periodico al Vocabolario della Crusca pubblicato da Alessandro Verri nel Caffe (I764), un energico attacco al fiorentino e al Vocabolario dell'Accademia della Crusca, bollato perché arcaizzante ed espressione di una cultura formalistica, paga delle parole e indifferente alle cose e alle idee. Dunque l'Accademia della Crusca non è apprezzata come custode gelosa della lingua e della sua tradizione, ma come un freno ai progressi della ragione: "gli autori del Caffe»» sono «estremamente portati a preferire

62 Da Alfredo Stussi, Scelte linguistiche e connotati regionali nella novella italiana [1989], ora in Id., Lingua, dialetto e letteratura, Torino, Einaudi, I993, pp. I 29-53, a p. I 52.

${ }^{63}$ Paradossi. Ristampa dell'edizione Lione I 543, Presentazione di Eugenio Canone e Germana Ernst, Pisa-Roma, Istituti Editoriali e Poligrafici Internazionali, I999, pp. I09-I0.

${ }^{64}$ Cfr. Giovanni Pozzi, L'italiano in chiesa [1995], in Id., Grammatica e retorica dei santi, Milano, Vita e pensiero, I997, pp. 3-46, che aiuta a comprendere il passo di Emanuele Tesauro citato da Claudio Marazzini, Da Dante alle lingue del Web, Roma, Carocci, 201 3, p. I07. 
le idee alle parole» e, si legge poco dopo, si preoccupano di «rendere meglio le loro idee». La convinzione è ribadita ancora: «è cosa ragionevole [razionale] che le parole servano alle idee, ma non le idee alle parole». ${ }^{65}$ Facendosi portavoce di un gruppo concorde, Verri fa «solenne rinunzia alla pretesa purezza della toscana favella ${ }^{66}$ e ai testi di lingua del Tre e del Cinquecento, riservandosi la libertà di usare parole non impiegate dagli scrittori antichi, e adottare termini estranei a Firenze e alla Toscana, raccolti da tutta Italia non meno che dalle lingue straniere:

perché se italianizzando le parole francesi, tedesche, inglesi, turche, greche, arabe, sclavone noi potremo rendere meglio le nostre idee, non ci asterremo di farlo per timore o del Casa o del Crescimbeni o del Villani o di tant'altri, che non hanno mai pensato di erigersi in tiranni delle menti del decimo ottavo secolo.

Lo spunto, caratteristicamente illuministico, sulla tirannide delle menti, va imputato non agli autori della tradizione, ai quali per altro Alessandro Verri non è interessato, ma all'organismo che li ha proposti come auctores: la Crusca, ovviamente, custode della lingua tradizionale, di cui Verri dichiara, con altra espressione che rende il linguaggio del tempo, di non voler «venerare gli oracoli», evidentemente arbitrari. ${ }^{67}$ Dice ancora Verri che un accanimento particolare è riservato alle regole ortografiche (segnalo con il corsivo le parole-bandiera del nuovo indirizzo intellettuale): ${ }^{68}$

${ }^{65}$ Cito da «Il Caffè» I764-I766, a c. di Gianni Francioni e Sergio Romagnoli, Torino, Bollati Boringhieri, I $998^{2}$, voll. 2, vol. I, pp. 47-50, alle pp. $47,48,49$.

${ }^{66}$ Ivi, p. 48 (corsivo dell'Autore).

${ }^{67}$ Ivi, p. 48. Diverso, come è noto, sarà in seguito lo sviluppo linguistico e intellettuale di Alessandro Verri (sul quale cfr. il libro recente di Leonardo Bellomo, Dalla "Rinunzia» alla Crusca al romanzo neoclassico. La lingua di Alessandro Verri in "Caffe» $e$ "Notti Romane», Firenze, Cesati, 2013).

${ }^{68}$ «Il Caffè», cit., p. 49. 
Porteremo questa nostra indipendente libertà sulle squallide pianure del dispotico Regno Ortografico e conformeremo le sue leggi alla ragione dove ci parrà che sia inutile il replicare le consonanti o l'accentar le vocali.

La ragione dovrebbe dunque occuparsi di consonanti doppie e di accenti; e alle consonanti doppie (classico problema ortografico - e fonetico - nell'Italia settentrionale) Alessandro Verri dedica un'attenzione particolare:

se il mondo fosse sempre stato regolato dai grammatici, sarebbero stati depressi in maniera gl'ingegni e le scienze che non avremmo tuttora né case, né morbide coltri, né carrozze, né quant'altri beni mai ci procacciò l'industria e le meditazioni degli uomini; ed a proposito di carrozza egli è bene il riflettere che, se le cognizioni umane dovessero stare ne' limiti strettissimi che gli assegnano i grammatici, sapremmo bensì che carrozza va scritta con due erre, ma andremmo tuttora a piedi. ${ }^{69}$

Per la verità non si capisce come la grafia, con una o due erre, possa incidere sulla carrozza; si capisce invece che il nobile illuminista Alessandro Verri è abituato a non andare a piedi, diversamente da Giuseppe Parini, che nobile non era e che dalle carrozze rischiava di essere investito. È chiaro per altro che l'articolo di Verri, di là dalla pura logica e, se si preferisce, dalla ragione, vale come rifiuto della tradizione linguisticoletteraria, e come polemica contro Firenze che, in età barocca e anche in seguito, non aveva tenuto il passo con Milano e la Lombardia, e perciò non poteva accampare pretese di tutela linguistica su Milano. Dopo tutto ciò, è singolare che nell'errata corrige annessa al primo volume la forma nodaro sia stata fiorentinizzata in notaio. ${ }^{70}$

\footnotetext{
${ }^{69}$ Ivi, pp. 48-49.

${ }^{70} \mathrm{Si}$ veda la documentazione nel commento dell'edizione citata, II, pp. I024-25. Da Milano a Venezia, la cultura linguistica ecletticamente con-
} 
7. Quarant'anni dopo, nella Milano napoleonica, il clima culturale è cambiato e il senso della tradizione fiorentino-italiana è riproposto da un'iniziativa editoriale che rilancia un'idea linguistico-letteraria unitaria, senza fratture con il passato. Nel I 802 si costituisce la Società Tipografica dei Classici Italiani, composta da tre soci: il banchiere Giovanni A. Borsa, l'ingegnere Innocenzo D. Giusti, il sacerdote Giulio Ferrario, che fra i tre è la persona effettivamente esperta nel merito culturale dell'impresa. ${ }^{71} \mathrm{Si}$ tratta di un'iniziativa imprenditoriale di natura privata che ottiene l'appoggio del governo nella persona di Francesco Melzi d'Eril, il vicepresidente della Repubblica Italiana. Per suo tramite, il governo s'impegna ad acquistare ottanta copie di ogni volume, e conferisce così prestigio e concreto aiuto alla serie dei volumi, pubblicati in tempi brevi da un gruppo di redattori destinato ad alimentare le migliori energie editoriali e intellettuali, che saranno attive anche nel periodo post-napoleonico.

ciliativa di Goldoni da un lato accosta il veneziano all'area lombarda, ma si guarda dal dissolvere l'idioma nativo in una vasta e non meglio definita area settentrionale: nella prefazione (I76I) al primo volume dell'edizione del teatro comico pubblicato a Venezia da Pasquali, e riferendosi al teatro in lingua, Goldoni scrive: «là dove sarò forzato di usare le parole o le frasi Veneziane o Lombarde, darò in piè di pagina la traduzione»; cito da Tutte le opere, a c. di Giuseppe Ortolani, Milano, Mondadori, I935-I956, I 4 voll. (I, p. 627); e sulle glosse che accompagnano varie commedie, e per altro, cfr. Gianfranco Folena, L'esperienza linguistica di Carlo Goldoni [1958], ora nel suo volume L'italiano in Europa, Torino, Einaudi, I983, pp. 89-I32, particolarmente le considerazioni e i rinvii della n. 7, pp. I 16 - I 7 7. Tre dei personaggi del Teatro comico sono contrassegnati da un asterisco, cui corrisponde questa avvertenza: «I tre Personaggi segnati colla * parlano il linguaggio veneziano, mescolato di qualche voce lombarda» (Tutte le opere, ed. cit., II, p. I047).

${ }^{71}$ Cfr. Marino Berengo, Intellettuali e librai nella Milano della Restaurazio$n e$, Torino, Einaudi, I980, pp. 8-2 I, e anche Roberto Tissoni, Il commento ai classici italiani nel Sette e nell'Ottocento (Dante e Petrarca), Padova, Antenore, I993, pp. I I4-I9. 
Nel 1803 i sottoscrittori dell'intera collana sono settecentotredici: alle seicentotrentadue copie per i privati vanno aggiunte le ottanta acquistate dal governo e quella sottoscritta da Napoleone in persona. Appena novantasei sono gli abbonati che non vivono nella Repubblica Italiana, un dato che mostra il forte potere separativo dei confini interni alla penisola.

Un manifesto del I 802 annuncia e pubblicizza il piano, che dodici anni dopo è già completato con la pubblicazione di ben duecentocinquanta, più esattamente duecentoquarantanove più un ultimo volume con il catalogo ragionato di tutta la serie. ${ }^{72}$ Dall'elenco si ricava un comodo sguardo d'insieme sulle scelte editoriali: i testi pubblicati vanno dalle origini al 1700 circa, lasciano fuori perciò gli ultimi cento anni della storia letteraria; forte è l'interesse per gli scrittori di storia. Il canone milanese degli autori italiani è modellato in gran parte sui testi, soprattutto fiorentini, posti a fondamento del vocabolario dell'Accademia della Crusca. È sensibile l'influenza della sistemazione storiografica della poesia italiana compiuta dal Crescimbeni, padre dell'Accademia dell'Arcadia (non per nulla ripudiato dalla Rinunzia di Alessandro Verri). Prevale dunque l'idea della gittata lunga di lingua e letteratura, che con i fiorentino-toscani abbraccia gli scrittori nativi di altre regioni che si siano adeguati ai modelli prevalenti; ed è importante, per la rappresentatività italiana della scelta, la rete nazionale intessuta dall'Arcadia. ${ }^{73}$ Dunque la Società milanese dei Classici Italiani ripropone il classicismo volgare della

\footnotetext{
72 Bibliografia od elenco ragionato delle opere contenute nella collezione de' Classici Italiani, Milano, Dalla Società Tipografica de' Classici Italiani, I 8 I 4. Un elenco conciso è in Bartolomeo Gamba, Serie dei testi di lingua $e$ di altre opere importanti nella italiana letteratura scritte dal secolo XIV al XIX, Venezia, Il Gondoliere, I $839^{4}$ (rist. anast., Torino, Bottega d'Erasmo, I965), pp. 74I-42.

${ }^{73}$ Cfr. Berengo, Intellettuali e librai, cit., p. I I, da cui ricavo anche altre indicazioni qui adunate.
} 
Crusca fiorentina e dell'Arcadia romana, anche attraverso il filtro di un dotto veneto, Bartolomeo Gamba, autore di un catalogo dei testi di lingua riprodotto nell'ultimo volume, e il gesto ribelle di Alessandro Verri si rivela il frutto di una stagione altra e diversa. ${ }^{74}$

Una conferma eloquente viene da un particolare minore e per ciò stesso significativo di uno degli autori pubblicati dalla Società dei Classici Italiani: nel I 809-1 8 I o escono in cinque volumi le Opere del Cavaliere Lionardo Salviati, fondatore dell'Accademia della Crusca: le Opere, non Tutte le Opere. Come avvertono Gli editori a' loro associati ed al colto pubblico:

È questa la prima volta, che vengono per nostro mezzo ristampate in un sol corpo tutte le opere del Cavaliere Lionardo Salviati, Scrittore sommamente benemerito dell'Italiana favella, ed uno de' principali ornamenti, anzi il fondatore della Fiorentina Accademia. Ma non tutte le opere del Salviati sono egualmente pregevoli ed interessanti. Alcune anzi ve n'ha fra di esse, dettate da critica troppo aspra, e che sebbene recar potessero qualche diletto ne' tempi, in cui il Salviati scrisse, nojose ora riescirebbero, e di niun profitto. Tali sono le opere ch'egli scrisse nell'occasione della guerra, che l'Accademia della Crusca dichiarò a Torquato Tasso, e che pubblicate furono sotto il nome dell'Infarinato nel i 585 e nel I 588 . [...] Queste $[\ldots]$ non avranno luogo nella nostra edizione. ${ }^{75}$

\footnotetext{
${ }^{74}$ Nella Prefazione all'edizione I 828 della sua Serie dei testi di lingua, riprodotta nell'edizione del I 839 cit. alla n. 7I, il Gamba (che era nato a Bassano), scrive: «trattasi di un bibliografico lavoro che comparve povero la prima volta l'anno i 805 da' torchi di Bassano, che meglio foggiato ricomparve l'anno i 8 I 2 da' torchi della Regia Stamperia di Milano, e che col titolo di Bibliografia de' Classici Italiani si volle a corredo d'Indice Generale nella Collezione di Opere italiane, pubblicata in Milano dall'anno I 804 all'anno I 8 I 4 , in 250 volumi in forma di ottavo" (p. v).

${ }^{75}$ Lionardo Salviati, Opere, Milano, Dalla Società Tipografica de' Classici Italiani, I 809, voll. 5, vol. I, pp. v-VI, con riproduzione dei corsivi usati nella stampa.
} 
La polemica fiorentinista di Salviati contro Tasso, al quale l'acre grammatico fiorentino attribuiva la stessa origine bergamasca (con le conseguenze denigratorie del caso) del padre Bernardo, fu dunque giudicata controproducente dalla collana milanese di primo Ottocento, che proponeva una letteratura in lingua italiana prevalentemente dei secoli XIV e XVI: modello di lingua, anche se invecchiata sul piano delle idee. ${ }^{76}$

Non per ciò il dualismo tra Milano e Firenze può considerarsi scomparso: riemerge a Milano negli ultimi anni della dominazione napoleonica e nei primi della Restaurazione austriaca. È questo l'episodio illuminato da Vitale nel suo studio, già ricordato $(\$ 2)$, Lombardi e toscani nella questione del Vocabolario. L'Istituto Nazionale di Scienze, Lettere ed Arti e l'Accademia della Crusca. Di fondazione napoleonica, l'Istituto, con sede a Milano e sezioni a Venezia, Bologna, Padova e Verona, ricevette nel I8 33 dal Ministro dell'interno del Regno italico l'invito, equivalente a un ordine, a concepire e organizzare un dizionario della lingua italiana; e a questo scopo fu nominata una commissione. Se alcuni soci, interessati alla lingua, producevano contributi settoriali di lessicografia ita-

\footnotetext{
${ }^{76}$ Invece, poco più di dieci anni prima della frattura, poi rientrata, di Alessandro Verri e della sua Rinunzia, il Torquato Tasso (I75I) del Goldoni aveva portato sulla scena, insieme al poeta, con la sua debolezza psicologica e con l'amore impossibile per una dama, anche una macchietta, «il Cavalier del Fiocco, l'acerrimo cruscante» (in rima con pedante; a. I, sc. II), riduzione caricaturale del Salviati, presentato come nemico letterario e personale del Tasso, che alla lingua della Liberata contesta tra l'altro l'eccesso dei latinismi e dei lombardismi (si tratta di un'accusa tradizionale). Questi e altri difetti fanno di Tasso un «lombardo ignorantissimo» (a. I, sc. Ix e x; Opere, ed. cit., v, pp. 779, 785). La marchesa Eleonora, della corte ferrarese, si esprime, riformulando inconsapevolmente ciò che aveva asserito Bandello: «Eे vero che i Lombardi non han lingua perfetta, | ma studiano gli autori, scelgon di loro il buono....» (a. I, sc. XI; ed. cit., p. 786). Con ciò, tuttavia, Goldoni non voleva certo rinfocolare le antiche polemiche, sulle quali nella Milano napoleonica si preferiva, invece, tacere.
} 
liana o studi d'interpretazione filologico-linguistica, l'Istituto nel suo complesso non era realmente attrezzato per compilare un dizionario di vasto respiro. ${ }^{77}$

Intanto, a Firenze, nel i 8 I I Napoleone aveva richiamato in vita l'Accademia della Crusca, con un'iniziativa piuttosto strumentale, che doveva servire a tacitare le preoccupazioni di molti toscani, che mal sopportavano l'energica francesizzazione imposta negli usi pubblici della lingua anche alla Toscana, sicché il futuro di Firenze come tradizionale centro linguistico del fiorentino-italiano era tutt'altro che assicurato. ${ }^{78} \mathrm{La}$ Crusca rinata si dava a studiare e pubblicare i testi del buon secolo della lingua, con una scelta culturale che ne confermava l'orientamento trecentistico ma risultava ben poco efficace sul piano del giornalismo, dell'amministrazione, degli usi pubblici dell'italiano; ciò però consentiva agli Accademici della Crusca di ribadire una sorta di privilegio e quasi di monopolio sulla lingua riservato alla Toscana e, con sottinteso evidente, alla Crusca, custode del tesoro fiorentino-toscano. ${ }^{79}$ Dal canto loro i soci dell'Istituto milanese coltivavano la convinzione della propria superiorità culturale rispetto a una Toscana nella quale il progresso filosofico e scientifico era stato molto meno sensibile; Firenze, infatti, era rimasta relativamente poco permeabile all'Illuminismo così come, nel xvir secolo, alla cultura

${ }^{77}$ Qui e in seguito mi servo del lavoro di Vitale, Lombardi e Toscani, cit.

${ }^{78}$ Sull'argomento è preziosa la ricerca di Piero Fiorelli, L'italiano, il francese, la Toscana e Napoleone, negli Studi in onore di Manlio Udina, Milano, Giuffrè, I975, voll. 2, vol. II, pp. I 579-1602.

${ }^{79}$ Vitale, Lombardi e Toscani, cit., pp. 547-50 e p. 492 n. 7, dove si ricorda, insieme con esplicite, significative testimonianze nel senso appena indicato (alle quali si può aggiungere un passo dello Zannoni riportato da Claudio Marazzini, L'ordine delle parole, Bologna, Il Mulino, 2009, p. 255), che nel I 823 Vieusseux, uomo estraneo a ogni sorta di particolarismi, dichiarava che l'Antologia non avrebbe accolto lavori ispirati alla polemica tra toscani e lombardi. 
barocca. Da quella superiorità culturale doveva risultare anche una superiorità sul piano di una lingua colta, che non si limitasse alla registrazione del parlato o di testi antichi, e sapesse nominare le nuove idee e le nuove scienze.

I distinti sviluppi culturali delle due città si incontrarono (e scontrarono) quando, dopo la caduta del regime napoleonico, l'idea del dizionario fu riproposta all'Istituto dalle autorità austriache, in continuità con l'orientamento di età napoleonica, e rincalzata dall'invito a chiedere la collaborazione dell'Accademia della Crusca. L'orgoglio lombardo per i progressi della cultura milanese si tradusse nella proposta di un vocabolario cui collaborassero i dotti di tutta Italia, guidati di fatto dai milanesi; l'opera avrebbe dovuto fondarsi sul lessico delle idee filosofiche e scientifiche moderne diffusesi nell'Italia moderna, non sui testi di lingua riproposti pochi anni prima, proprio sull'esempio della Crusca, anche a Milano dalla collana di cui si è detto. L'invito ai dotti italiani, poi, conferiva all'iniziativa un orizzonte nazionale e quindi una larghezza di vedute assai più ampia del nobile ma circoscritto municipalismo fiorentino della Crusca, cui nell'impresa si assegnava di fatto il ruolo di una fra le tante collaborazioni. Le pretese di egemonia culturale sottese alla proposta milanese, e la diversa impostazione linguistico-culturale spiegano come la Crusca rifiutasse la proposta (I 8 I 6); e anche senza insistere sulla poca voglia di far confluire le energie per un fine comune, né Milano né Firenze erano davvero in grado di produrre lo sforzo collettivo di un grande vocabolario. Sfumata la proposta di collaborazione, avanzata dall'Istituto di Milano con più di una riserva mentale, l'ipotesi della convergenza cedeva alla brillante satira della Proposta di alcune correzioni ed aggiunte al Vocabolario della Crusca (I 8 I7-I 824) di Monti e dei suoi collaboratori. La Proposta prendeva di mira la Crusca veronese del Cesari, e dava luogo a un nuovo episodio di accesa polemica tra lombardi e toscani. 
8. La questione dei lombardi e dei toscani si ripropone lungo l'Ottocento in termini nuovi. Mentre uscivano gli ultimi volumi della Proposta del Monti, Manzoni si cimentava nel Fermo e Lucia (1 82 I-23), per il quale scriveva una prima introduzione, poco dopo aver avviato la scrittura dell'opera e poi, a libro finito, una seconda introduzione, ricca di indicazioni sul divenire dello scrittore e del suo pensiero linguistico, stimolato dalle esigenze di quel genere nuovo per l'Italia che era il romanzo. Giunto infatti al termine del Fermo e Lucia, l'autore riflette sulla riscrittura dell'Anonimo seicentesco e sulla lingua del libro, dichiarando francamente: «noi abbiamo conservate non solo nei dialoghi, ma anche nel racconto vocaboli, modi proverbiali, frasi assolutamente lombarde». ${ }^{80}$ Peggio ancora, prosegue lo scrittore, il romanzo è

un composto indigesto di frasi un po' lombarde, un po' toscane, un po' francesi, un po' anche latine; di frasi che non appartengono a nessuna di queste categorie, ma sono cavate per analogia e per estensione o dall'una o dall'altra di esse. ${ }^{81}$

Dunque il lombardismo convive con il toscanismo, il gallicismo, il latinismo, la coniazione analogica: non si ripresenta perciò l'alternativa polemica toscani-lombardi, perché il problema è l'insufficienza o l'inadeguatezza, a Milano come altrove, di una lingua nazionale. Firenze non è chiamata in causa in modo esplicito, ma implicitamente Manzoni non può

${ }^{80}$ Cito dalla nuova edizione dei Promessi sposi I 82 I-I 827 , diretta da Dante Isella, Prima minuta (I82I-I823). Fermo e Lucia, a cura di Barbara Colli, Paola Italia e Giulia Raboni, Milano, Casa del Manzoni, 2007 $7^{[2]}$, voll. 2, vol. I, p. 5, dalla quale risulta che la seconda introduzione al Fermo $e$ Lucia è ricca di correzioni, e che dal suo tronco si staccò un ramo collegato con il resto; il testo non fu omogeneizzato, e rimase incompiuto, perché le idee dell'autore erano in movimento, sicché l'interessantissimo scritto fu abbandonato.

${ }^{81}$ Ivi, I, p. 6. 
che constatare l'inesistenza, in Italia, di "parole e frasi adottate esclusivamente per convenzione generale»: ${ }^{82}$ un uso linguistico nazionale, dunque, non è stato garantito dal toscano.

Dalle premesse problematiche che informano il Fermo $e$ Lucia muove una nuova, lunga meditazione teorica sull'italiano e sulla natura delle lingue in generale che, prima dell'Unità, portò Manzoni a pubblicare Sulla lingua italiana. Lettera a Giacinto Carena ( 1847 ) nella quale indicava nel fiorentino dell'uso la soluzione del problema linguistico nazionale, e i Promessi sposi. Dopo l'Unità, il pensiero linguistico, molto prestigioso ma non da tutti condiviso, di Manzoni, assumeva una dimensione non solo culturale ma pubblica, con effetti sulla politica linguistica del Regno d'Italia, grazie alla relazione Dell'unità della lingua e dei mezzi di diffonderla (I 868) (33 $^{83}$ e soprattutto al suo effetto pratico, il Novo vocabolario della lingua italiana, del I870-I897, citato comunemente come Giorgini-Broglio, espressione del fiorentinismo di Manzoni. ${ }^{84}$

Con la posizione linguistica di Manzoni e il celebre attacco di Ascoli, nel Proemio all' "Archivio Glottologico Italiano»

\footnotetext{
${ }^{82}$ Ivi, I, p. 8.

${ }^{83}$ Si legge negli Scritti linguistici, a c. di Angelo Stella e Luca Danzi, in Scritti linguistici e letterari, t. II, Milano, Mondadori, I990 (A. Manzoni, Tutte le opere, a c. di Alberto Chari e Fausto Ghisalberti, Milano, Mondadori, vol. v), pp. 603-25; e si veda ora l'attenta, ricca analisi di Angelo Stella, Appendice manzoniana al Manzoni, in Graziadio Isaia Ascoli. Convegno nel centenario della morte (Roma, 7-8 marzo 2007), Roma, Accademia Nazionale dei Lincei, 2010, pp. 243-307. Una nuova edizione è Dell'unità della lingua e dei mezzi di diffonderla. Edizione critica a cura di Claudio Marazzini e Ludovica Maconi, [Roma], Società Dante Alighieri, 20 I I.

${ }^{84}$ Se ne ha una ristampa anastatica in quattro volumi, Firenze, Le Lettere, I979, con importante introduzione di Ghino Ghinassi che mostra come l'opera, poco diffusa e fortunata per la sua mole e per altre ragioni, fu tuttavia il modello dei dizionari che entrarono nelle classi scolastiche dell'Italia Unita.
} 
del 1873 , il dualismo di lombardi e toscani rivive in modi nuovi e inediti, che tuttavia si lasciano anche ricondurre alla lunga tradizione di cui si sono ripercorsi qui alcuni episodi. La parte dei toscani è difesa dal milanese Manzoni: non già nei modi del tradizionale fiorentinismo dell'Accademia della Crusca, ma in chiave decisamente nazionale e non municipale; per non dire che l'avere spezzato il binomio di uso e tradizione letteraria, al quale la cultura fiorentina era stata fedele nel tempo ${ }^{85} \mathrm{fu}$ accolto sfavorevolmente dalla sezione fiorentina della Commissione nominata dal ministro Broglio.

In margine alla relazione (quasi una contro-relazione) inviata dalla commissione di Firenze, Manzoni scrisse una lunga, ricca Appendice alla Relazione (I 869) nella quale l'anziano scrittore sa trarre dalla novità della rete ferroviaria che si andava costruendo nell'Italia postunitaria un esempio efficace: si tratta di documentare un aspetto della frammentazione dell' $i$ taliano cui la scelta coerente del fiorentino dovrebbe porre rimedio. Un francese, dunque, avrebbe notato la «mancanza in Italia di nomi comuni per significare cose comuni», e chiesto spiegazioni a un amico di Manzoni «che si trovava in un vagone con lui, andando da Milano a Firenze». ${ }^{86}$ Manzoni riporta la lettera inviatagli dall'amico:

Il mio interlocutore era un giovinotto francese di bonissimo garbo, che non era mai stato in Italia, e giacché c'era venuto, voleva almeno spenderli giustificati. Osservava, interrogava e notava in un suo taccuino che era sempre in ballo. Durante la fermata del treno a Pistoia, mi domandò che cosa voleva dire una parola dipinta in verde a gran caratteri sopra una porta. La parola era egresso. Risposi

${ }^{85}$ Il binomio riaffiora nella relazione della sottocommissione fiorentina, che non fu consultata da Manzoni e, dopo la pubblicazione della relazione milanese, inviò al ministro Broglio una relazione sua propria, per più aspetti evidentemente anche se implicitamente divergente da Manzoni (cfr. gli Scritti linguistici, cit., pp. 642-52).

${ }^{86}$ L'Appendice è negli Scritti linguistici, II, cit., pp. 703-77 (p. 763). 
che voleva dire sortie. Tirò fuori il taccuino, e dopo averlo consultato, soggiunse: - Alla stazione di Milano c'è uscita. - E se avrete, soggiunsi io, la pazienza d'arrivare a Firenze, alla Stazione di Firenze, troverete sortita; e non c'è nessuna ragione perché andando più in là non troviate, esito, uscimento, evacuazione o che so io ${ }^{87}$.

L'amico del Manzoni (che è forse il Giorgini) precisa che nessuno di quei nomi trascrive la parola del dialetto locale: «a Milano nella lingua, o se volete nel dialetto del luogo, non si dice uscita, né a Pistoia egresso, né a Firenze sortita»: ${ }^{88}$ sono l'effetto di un serbatoio lessicale ricco di sinonimi (segno, nella concezione manzoniana, di povertà), cui attingono in modo casuale gli uffici di uno stato che pure aveva un ordinamento tendenzialmente centralizzato. Anche dalla tratta ferroviaria MilanoFirenze, dunque, Manzoni ricavava argomenti per difendere un'idea del fiorentino che non convinceva la commissione di Firenze, e che a Milano aveva adepti ma anche oppositori; tra questi il più energico era certo l'Ascoli.

La parte dei lombardi e forse, più esattamente, di Milano, è rappresentata invece da un milanese d'adozione, il goriziano Graziadio Ascoli. Due protagonisti milanesi, dunque; e il fautore di Milano rigetta la dottrina manzoniana, non meno che i criteri del Novo vocabolario, e rifiuta il primato linguistico della Toscana, "una terra così fertile d'analfabeti». ${ }^{89}$ Senza entrare nel merito di aspetti più noti (il ruolo della lingua scritta in Ascoli di contro al ruolo della lingua parlata e scritta in Manzoni) e meno noti (il rapporto tra lingua e pensiero, che convergono e quasi s'identificano in Ascoli, diversamente da Manzoni) che stanno a fondamento del deciso antimanzonismo ascoliano, ricordo l'avvio del Proemio, e la critica del nòvo

\footnotetext{
87 Scritti linguistici, II, cit., pp. 763-64.

${ }^{88}$ Scritti linguistici, II, cit., p. 764.

${ }^{89}$ G. I. Ascoli, Scritti sulla questione della lingua, a cura di Corrado Grassi, Torino, Einaudi, I975, pp. 28-29.
} 
che figura nel titolo del Giorgini-Broglio. Ascoli spiega che dalla $o$ breve latina (in sillaba libera) si apre il dittongo $u o$ tonico, mentre l'esito della $o$ lunga latina è $o$ tonica chiusa; in nota osserva che una distinzione analoga tra vocale breve e lunga

si riproduce, in modi diversi, anche in un numero infinito di altri vernacoli italiani. Così, per esempio, l'o breve latino è l'oe di Milano: noef, moef, moer, coer, roeda, ecc; laddove l'o lungo latino è l'ó di Milano: vóć, famós, colór, patrón, sióra, ecc. E similmente all'altra estremità dell'Italia, l'o breve latino è sempre $o$ a Palermo: nòvu, lòcu, focu, jòcu, scòla, còciri, ecc.; laddove l'o lungo latino è sempre $u$ a Palermo: sùli, amùri, siñura, cùda, scùpa, ecc. ${ }^{90}$

Si noti come Ascoli parta da Milano e si trasferisca mentalmente e linguisticamente all'altra estremità dell'Italia, a Palermo. Si veda ora un passo del Proemio in cui sono comparate tre locuzioni sinonimiche in toscano, in veneziano e in siciliano, con un'equa distribuzione tra aree linguistiche settentrionali, centrali e meridionali:

nessuno avrebbe mosso rimprovero al veneziano se egli offriva alla letteratura italiana il suo mettere il cervello a segno, malgrado il pericolo che a Firenze così non si dicesse, o ivi piuttosto mettessero il cervello a bottega od a partito. Vero è che il siciliano, per significare il medesimo, sarebbe forse uscito col suo metter pensiero (mèttiri pinsèri), unione di parole che altrove può valere "dare apprensione”. Ma ognun vede, dopo il primo sgomento, che l'equivoco non può facilmente avvenire od anzi è a dirittura impossibile; poiché "metter pensiero", quando porti il significato di "dare apprensione", deve reggere di necessità un dativo, che nell'altra significazione deve di necessità mancare; e il metter pensiero, locuzione parallela al metter radice, sostenuto da una Sicilia che emulasse in attività civile la Sassonia, cioè che mandasse al continente italiano i suoi milioni di chilogrammi di libri, avrebbe potuto fare ben legittima fortuna, poiché l'autorità legittima è l'energia operosa ${ }^{91}$.

\footnotetext{
${ }^{90}$ Ivi, p. 6 n.

${ }^{91}$ Ivi, p. 22.
} 
Ascoli immagina una gara di attività scientifica tra la Sassonia e la Sicilia, e però sono convinto che Ascoli scriva Sicilia ma mentalmente si collochi all'altra estremità dell'Italia e insomma intenda la sua Milano come avanguardia del progresso intellettuale italiano, di contro alla Toscana: di nuovo Milano come centro guida del paese, come negli anni a cavallo tra la Milano napoleonica e la Milano della Restaurazione.

Non so se dopo questo episodio si possano registrare altri significativi momenti del confronto lombardo-toscano: certo è che dopo l'Unità se ne affermerà uno diverso, tra la Milano dell'economia (e talvolta capitale morale del paese) e la Roma della politica. Ma questa è un'altra storia.

9. Alcuni anni fa mi è avvenuto di retrodatare di circa un quarto di secolo la comparsa di fiorentinità: la prima attestazione nota della parola è infatti, secondo i dizionari storici, nell'Ercolano di Benedetto Varchi, il lungo dialogo sulla lingua cui l'autore lavorò fino alla morte (1565) e fu pubblicato postumo (I 570). In latino, un'attestazione anteriore si legge in una lettera, edita ma poco conosciuta, dell'umanista Giovanni Corsi, cui Guicciardini, suo amico, aveva dato da leggere la Storia d'Italia. Corsi risponde (forse nel I 538 - I 539 , comunque prima del I 540, anno della morte di Guicciardini) lodando l'opera e avanzando qualche riserva sulla sua veste formale, non del tutto adeguata alla precisa conoscenza dei fatti per la quale Guicciardini supera di gran lunga tutti gli altri storici; lo stile è grave, le orazioni e i discorsi indiretti eleganti e degni dell'eloquenza di Livio. Tuttavia la scrittura e la scelta delle parole si allontanano talora dalla solennità senatoria per scendere in piazza, sicché l'opera manifesta un sapore troppo acuto di florentinitas, e scarseggiano i colori delle figure e le metafore. Il latino conservava una sua capacità creativa, e Corsi conia una florentinitas capace di assorbire il significato positivo dell'urbanitas della retorica classica e quello critico 
della patavinitas riscontrata da qualche lettore antico nella storia di Livio. ${ }^{22}$

Una nuova occorrenza di florentinitas, indipendente da quella ora menzionata, si trova nell'Hypercalipsis. Nel I 809 Pindemonte pubblica un volume comprendente la traduzione dei primi due canti dell'Odissea; di qui prende lo spunto Foscolo per pubblicare l'anno dopo, sugli «Annali di Scienze e Lettere» diretti dal suo amico Rasori, un articolo Sulla traduzione dell'«Odissea». Pindemonte riceve qualche lode e qualche garbata riserva, ma in realtà il saggio di Foscolo, privo di una linea discorsiva coerente, si occupa, come scrive verso la fine lo stesso recensore, de rebus omnibus et de quibusdam aliis, ${ }^{93}$ risolvendosi in una serie di digressioni eterogenee in materia di traduzioni dal greco e insomma in un pretesto per criticare con virulenza alcune personalità: da Anton Maria Salvini, accademico della Crusca e grecista, a Melchiorre Cesarotti, fino a pochi anni prima nume tutelare della cultura padovana, ad Algarotti, perfino a Parini; né mancano allusioni critiche e spesso criptiche ad autori che restano innominati (tra i quali Vincenzo Monti). Non è strano che nei salotti, nelle lettere private, nella stampa l'articolo di un Foscolo solo contro tutti (e a tutti superiore) accendesse a Milano una polemica letteraria. Foscolo reagì con altri testi polemici, che rimasero inediti; tra questi l'Hypercalipsis, l'originale satira di stile biblico pubblicata, dopo la fuga da Milano, a Zurigo, in un momento di massima precarietà entro una vita che fu sempre agitata. Nell'operetta Foscolo si esibisce in una delle esibizioni fantabibliografiche nelle quali è maestro, e sfrutta

92 Francesco Bruni, «Fiorentinità» e «florentinitas». Una scheda per il lessico intellettuale cinquecentesco, "Lingua e Stile», 39 (2004), pp. 4564 .

${ }^{93} \mathrm{Si}$ veda il saggio foscoliano in Lezioni, articoli di critica e di polemica (I809-I8II), a cura di Emilio Santini, Firenze, Le Monnier, I933, pp. I97-230, a p. 228. 
i suoi alter ego letterari. ${ }^{94}$ Il frontespizio recita: Didymi Clerici prophetae minimi Hypercalypseos liber singularis; dunque l'opera è firmata da Didimo Chierico, lo pseudonimo dietro il quale Foscolo si era nascosto quando, nel I8 I3, aveva pubblicato il Viaggio sentimentale di Yorick lungo la Francia e l'Italia, facendo di Didimo Chierico il traduttore e l'editore del romanzo di Sterne; ${ }^{95}$ di Didimo, però, si legge che mancano notizie, non si sa neppure se sia vivo o morto. Editore dell'Hypercalipsis è allora "Laur[entius] Alderanus» cioè il «Lorenzo $A^{* * *}$ » editore delle Ultime lettere di Jacopo Ortis del i 80 i e del I 802, nella forma piena di Lorenzo Alderani editore dello stesso romanzo nell'edizione di Londra I 8 I 4 (in realtà Zurigo I 8 I 6: anno di pubblicazione dell' Hypercalypsis, presso lo stesso stampatore, il Füssli).

Come ben noto, Didimo Chierico è detto autore dell'Hypercalypsis; quanto a Lorenzo Alderani, già amoroso collettore ed editore delle lettere di Ortis suicida, nella dedica a un inesistente patrizio inglese riferisce di aver avuto da Didimo il permesso di copiare l'Hypercalipsys, a patto di non renderla di pubblico dominio, perché Didimo voleva evitare che il testo, documento negativo del rissoso costume letterario italiano, offrisse un nuovo esempio di contrasti tra scrittori. Oggetto di un interesse quasi morboso da parte del pubblico, un'opera polemica attira gli editori spregiudicati, pronti a dare in pasto al pubblico uno spettacolo miserevole, dal quale si ripromettono un guadagno. È accaduto, però, che un grammatico abbia volgarizzato e commentato parte dell'operetta di Didimo, sicché Lorenzo Alderani si sente sciolto dall'obbliga-

${ }^{94}$ Cfr. Maria Antonietta Terzoli, Foscolo, Roma-Bari, Laterza, 200o, pp. I $40-44$.

95 Didimo è infatti il titolare della dedica (Didimo Chierico a' lettori salute); in coda alla traduzione, una Notizia intorno a Didimo Chierico (Prose varie d'arte, a cura di Mario Fubini, Firenze, Le Monnier, I95 I, pp. 39-40, I73-86), che è riprodotta anche al termine dell'Hypercalypsis. 
zione di tenerla presso di sé. L'inventiva di Foscolo si spinge fino a definire la qualità dell'inesistente traduzione, in termini che riflettono le sue esperienze tra Milano e Firenze, e il dualismo di lombardi e toscani: "Quamquam in illa paraphrasi neque paginam, neque fortasse lineam reperies, quin Insubrem idiotismòn redoleat; interdum etiam Florentinitatem quaesitam: quod sane indicium Insubris grammatici est». ${ }^{96}$ Dunque il volgarizzamento risentirebbe di un colore municipale (per ripetere una felice espressione della seconda introduzione al Fermo e Lucia) lombardo e di un' esibizione fiorentina che per essere esibita si palesa, per ipercorrettismo, non fiorentina. ${ }^{97}$ Di qui Foscolo-Alderani prende lo spunto per polemizzare con la cultura linguistica fiorentina, prona al servilismo gallicizzante (cfr. la n. 78), non meno che contro la milanese, di matrice arcaizzante (e in questo caso il riferimento è alla collana dei classici italiani di cui si è detto al $\$ 7$ ).

La florentinitas del Foscolo è certo una riconiazione, perché la lettera del Corsi fu pubblicata solo nel i919; probabilmente fu stimolata da qualche testimonianza in lingua volgare del sedicesimo o, piuttosto, del diciottesimo secolo, e con ogni probabilità fu indotta dall'episodio delle controversie tra lombardi e toscani studiato nel contributo di Maurizio Vitale da cui hanno preso le mosse gli appunti qui riuniti.

\footnotetext{
${ }^{96}$ Cito da Ugo Foscolo, Opere, II, Prose e saggi, ed. dir. da Franco Gavazzeni, con la coll. di Gianfranca Lavezzi, Elena Lombardi, Maria Antonietta Terzoli, Torino, Einaudi-Gallimard, I995, pp. 358-59.

${ }^{97}$ Ricordo l'aneddoto di Teofrasto citato, fra gli altri, da Castiglione $(\$ 5$ ) o, per dirla con l'endecasillabo coniato subito dopo: «Troppo Toscano il non Toscano accusa» (Hypercalypsis, ed. cit., p. 359).
} 


\section{Vittorio Spinazzola}

\section{LA POLITICITÀ DEI «PROMESSI SPOSI»}

In uno scritto del I990, Maurizio Vitale pone a raffronto, con la consueta incisività, Manzoni e Dante, per sostenere che "l'importanza dell'opera di teorico della lingua e di scrittore di Alessandro Manzoni è comparabile solo a quella di Dante». Non si tratta dunque solo di un confronto e quasi un pareggiamento di valori estetici nei capolavori dei due scrittori, quanto piuttosto del riscontro di una analogia nelle rispettive fisionomie intellettuali: in entrambi i casi la vocazione alla creatività letteraria si congiunge all'impegno forte per lo studio delle questioni linguistiche, secondo un'ottica che oggi forse diremmo d'indole funzionalistica: l'attività di elaborazione testuale è chiamata a tenere conto di tutte le esigenze maturate nel cosmo sociale di appartenenza.

In questa prospettiva, afferma il grande linguista milanese, «il Manzoni si mostra un teorico acuto nel proporre soluzioni coerenti ai mutati problemi linguistici della società ottocentesca e un artista inarrivabile nel creare, con la sua mirabile prosa di romanzo faticosamente conquistata, un linguaggio nuovo nella storia letteraria e civile italiana». E l'accostamento della personalità manzoniana alla figura dantesca, caratterialmente così diversa, pone in rilievo un fatto decisivo: la loro riconoscibile fratellanza trova fondamento in una comune intentio auctoris di tipo suasorio, per non dire ideologico. Sia il romanzo storico ottocentesco sia il poema narrativo del Trecento intendono giovare alla recristianizzazione del mondo per loro moderno, con un'opera di evangelizzazione immaginosa. Perciò appunto sia l'uno sia l'altro scrittore esibiscono una visuale totalizzante delle istituzioni del viver collettivo che auspicavano cambiassero nel profondo: in altri 
termini, entrambi mostrano di riferire il loro immaginario poematicamente o prosasticamente realistico a una istanza politica religiosamente concepita.

In una sia pur succinta focalizzazione dei due casi è però opportuno un chiarimento di metodo preliminare: la distinzione netta fra i concetti di politicità letteraria e di letteratura politica. Il primo infatti indica un connotato immanente a qualsiasi impresa intellettuale destinata a incidere sullo stato delle relazioni interpersonali e sui canoni di comportamento vigenti in una determinata formazione di civiltà. Siamo dunque su un piano di oggettività socioculturale ineludibile, anche da parte di chi non nutra alcun interesse politico in senso specifico: ogni romanzo implica una esemplificazione delle modalità di rapporti dell'io singolo con l'essere collettivo. Il secondo concetto invece ha un'indole più restrittiva, in quanto riguarda la consapevolezza soggettiva dell'autore, nel suo proposito di svolgere un discorso che investa esplicitamente gli ordinamenti del potere, gli apparati statali, le strutture legislative secondo un punto di vista partigiano.

La politicità immanente a qualsiasi testo letterario risiede anzitutto nei procedimenti di lavoro adottati dallo scrittore, cioè nelle norme di linguaggio, nei criteri di tecnica rappresentativa cui si è attenuto. La loro elaborazione infatti è sempre funzionale al progetto di entrare in rapporto e captare il consenso di gruppi e strati di interlocutori storicamente, socialmente, culturalmente determinati. Appunto per questo, pubblicare un libro è comunque un atto a connotato politico, giacchè implica la scelta del pubblico elettivo cui rivolgersi, dotto o indotto, tradizionalista o innovativo. È questa opzione primaria a decidere la strumentazione espressiva cui dare luogo, con un grado maggiore o minore di originalità inventiva e coerenza sistematica.

La politicità letteraria dei Promessi sposi obbedisce in prima istanza alla deliberazione di scrivere un "libro per tutti", teso 
ad aprire un dialogo non solo coi ceti umanistici qualificati ma con il largo pubblico "non letterato, né illetterato", come chiarisce il Manzoni stesso, costituito dalla nuova classe dirigente che si affacciava alla scena storica. Stile, canoni di genere, caratterizzazione dei personaggi, modi d'intreccio, tutto è funzionale all'obiettivo di catalizzare la genesi di un pubblico allargato, che in concreto aveva la fisionomia della borghesia in via di sviluppo nel centro urbano milanese.

A ciò il Manzoni era spinto proprio dallo zelo religioso, che gli indicava un grande compito: moltiplicare le risorse del suo ingegno di scrittore ponendole al servizio di una impresa di riconquista cattolica del mondo moderno. La parola letteraria doveva attingere la massima affabilità nell'atto di improntarsi all'accento sacrale del Verbo. Il linguaggio profano della civiltà borghese veniva dunque a essere riplasmato con perizia eccezionale, appunto per riportarlo a uno spirito di verità rivelata, immutabile nei tempi.

Nell'organismo stilistico dei Promessi sposi, così leggibile e così raffinato, c'è tuttavia una contraddizione: il Manzoni rinunzia in realtà a rinnovare, personalizzandolo, il linguaggio della fede: si limita a intonarlo secondo i moduli tradizionali della catechesi. Quando il narratore esalta in modo più diretto il sentimento religioso, si ha un calo di originalità nella tensione espressiva, e a prevalere è una fraseologia retorica già ben collaudata nella letteratura sacra. Il lettore laico rilutta a riecheggiarla, interiorizzandola. Così il pubblico universale cui lo scrittore ambisce rivolgersi rischia di assumere le sembianze di un pubblico ecumenico, già intimamente e quasi ancestralmente partecipe di un credo confessionale che occorre soltanto riproporgli, con suadente fervore apologetico.

La discontinuità tra linguaggio narrativo e linguaggio apologetico riverbera su tutti i livelli di composizione testuale del capolavoro manzoniano: anzitutto le strutture di racconto. I Promessi sposi assumono la forma romanzo come la più tipica 
della modernità borghese, in quanto promette un colloquio più cordiale con la generalità del pubblico, intrattenendolo $\mathrm{e}$ avvincendolo con la raffigurazione di circostanze in cui chiunque possa sentirsi coinvolto. In particolare Manzoni sceglie il genere del romanzo storico perché l'intreccio fra grandi eventi pubblici e modesti fatti d'invenzione gli consente meglio di metter a fuoco il nodo centrale delle sue preoccupazioni: il rapporto sempre drammatico tra i diritti di libertà dell'uomo e del cittadino, anzitutto lo jus nubendi, e i doveri di responsabilità morale, sociale, civile verso i propri simili.

Questo rapporto eminentemente politico viene strutturato in una trama romanzesca che intende offrire una esemplificazione esauriente dei termini in cui si svolge sempre l'esperienza esistenziale, secondo un proposito di totalità rappresentativa organica. A muovere la macchina narrativa sono alcuni personaggi appartenenti alle classi subalterne della Milano secentesca. Ma le loro peripezie si proiettano in una dimensione sempre più vasta, secondo una tecnica di allargamento prospettico per onde concentriche e inserti digressivi, sino a incontrare, coinvolgere, attraversare tutti i modelli della vita di relazione in una civiltà preborghese, col suo assetto di disordine costituito.

La dimensione politica rappresenta insomma il vero presupposto su cui si impianta la vicenda romanzesca. Manzoni non è, dal punto di vista biografico, un intellettuale engagé, come tanti altri scrittori risorgimentali: ma ha una lucidità senza pari nell'inquadrare il problema del rapporto fra governanti e governati, fra il potere statale e i cittadini, come soggetti singoli e come ceti, corporazioni, caste. L'energia dinamica del libro proviene da una accettazione piena dei principi del liberalesimo politico e liberismo economico. Nessun dubbio quindi che il regime borghese sia infinitamente superiore a quello gentilizio, in quanto più vicino a una normativa cui il romanziere attribuisce un crisma di naturalità suprema. 
Il progresso della civiltà implica un doppio assunto: il destino dell'individuo è sempre connesso alla sorte della collettività di appartenenza; ma allora, o si è liberi tutti o non è libero nessuno. L'equanimità delle leggi non può ammettere mezze misure.

L'atteggiamento politico manzoniano ha però un altro versante, assolutamente coessenziale. Sta sempre e solo all'io singolo decidere se agire per il vantaggio comune o rinserrarsi nel culto dei propri interessi particolari. La facoltà di scelta fra il bene e il male è patrimonio inalienabile di ogni coscienza. Manzoni vuole portare a coincidenza il credo del liberalesimo con la dottrina del libero arbitrio, e proprio perciò vede nella politica la dimensione operativa di ogni sforzo di umanizzazione dei rapporti interpersonali. Ma nello stesso tempo egli la deprime e umilia senza scampo. Ai suoi occhi, essa non è che il regno perenne della corruzione, della frode, del sopruso. L'unica garanzia di rispetto per i diritti originari della persona è fornita dai precetti religiosi di cui l'istituzione ecclesiastica è depositaria per mandato divino. Fuori della Chiesa non c’è salvezza, né per i cittadini né per gli stati.

La convivenza civile appare destinata a rimanere un caos inestricabile di insensatezze dolorose, se non ci si fortifica attraverso il ricorso alle certezze della fede. Torniamo così alla premessa prima e alla conclusione ultima dell'apologo romanzesco: l'invito perentorio a condividere un messaggio di verità rivelato metafisicamente. I Promessi sposi non potrebbero essere pensati fuori di questo afflato missionario, che ne pervade la compagine e ne sorregge la struttura. Il romanziere infatti non si limita a proclamare la necessità della trascendenza cristiana. Nega ogni riconoscimento positivo a qualsiasi esperienza di vita morale non riconducibile con immediatezza all'alveo delle dottrine custodite dalla Chiesa.

In questa luce si inquadra l'accanimento posto nel deprezzare ogni ipotesi di valore autonomo della politica. Come 
nell'Adelchi, nei Promessi sposi essa è soltanto l'arte di mascherare l'uso feroce della forza con una parvenza di diritto. Il narratore non concede alcuna nobiltà d'intenti, e nemmeno dignità di atteggiamenti, alla categoria dei politici, agli esponenti del potere laico, grandi o mezzani o piccoli, accomunati nella stessa condanna sprezzante e inappellabile. Il timor Domini è l'unico inizio non solo della sapienza morale, sì anche dell'avvedutezza politica.

Per rendere plausibile questo intransigentismo, Manzoni attua uno stratagemma geniale: preseleziona i personaggi narrativi, in modo che sulla scena romanzesca non compaiano, mai, figure nelle quali non traluca, per quanto obliterata, la presenza attiva dell'insegnamento evangelico. In effetti $I$ Promessi sposi non offre il resoconto di alcun conflitto tra religione e irreligione, fideismo e laicismo. La vera questione da dirimere che il libro prospetta è tutta interna alla cristianità: riguarda il contrasto tra chi vuol vivere integralmente il messaggio cristiano, nella sua luminosità perenne, come il cardinal Federigo o come fra' Cristoforo, e chi lo mistifica, compromettendolo nelle preoccupazioni mondane, come don Abbondio o il padre provinciale. Ai puri di cuore spetta di portare la loro innocenza disarmata, ma non inerme, al cospetto di Dio. Fede, speranza e soprattutto la virtù attiva della carità pongono nella disposizione migliore per trarre profitto da quegli eventi inattesi, casuali se non provvidenziali, che si producono nelle cose umane e lasciano aperto un varco alla fiducia nella possibilità di affermazione dei valori di una socialità positiva.

Il disegno di esperire una conciliazione tra cattolicesimo e mondo moderno non produce insomma una vera sintesi: e non per difetto ma per eccesso di motivazioni. Lo scrittore infatti giustappone un liberalesimo rigoroso a un integrismo confessionale perentorio. Diventa allora inevitabile che il secondo termine prevarichi irreversibilmente sul primo. In que- 
sto senso, I Promessi sposi è un libro eminentemente antipolitico: in nessun caso però apolitico. Si può anche sostenere che Manzoni si limita a aggiornare e riargomentare l'affermazione del primato dell'etica sulla politica, irrigidendolo. L'operazione tuttavia implica una scepsi analitica sui criteri e modi di gestione della cosa pubblica, da cui c'è tutto da imparare, pur se svolta in chiave di contestazione unilaterale. Pochi scrittori italiani hanno mostrato altrettanta perspicacia e competenza nell'illuminare i meccanismi del dominio politico, esemplati sulle vicende della Lombardia neofeudale.

I Promessi sposi esprimono l'auspicio di un risorgimento integralmente cristiano: ossia di una rivoluzione dei rapporti interpersonali che coincida con una restaurazione della fede avita. Questo è il punto di forza del libro, ma anche di debolezza. Manzoni non svolge una riflessione sui problemi teologici, né manifesta propositi strutturati di renovatio Ecclesiae: si limita ad affermare la necessità di presenza della Chiesa nella storia, in quanto solo la comunione ecclesiale offre asilo a quei diritti civili che l'essere collettivo calpesta. Non basta. Ogni istituzione insediata sulla terra, sia pure su mandato divino, risente della fallibilità intrinseca di tutti gli sforzi volti a ordinare le cose umane secondo canoni di giusta giustizia. Anche la Chiesa, nel suo assetto organizzativo, nei suoi rapporti di potere gerarchico, è sottoposta a una tensione lacerante: quanto maggiore è l'autorità esercitata da un uomo sui propri simili, tanto più forte è la tentazione, il rischio di orientarla verso mete sbagliate.

L'antiautoritarismo personalistico di Manzoni perviene alle conseguenze estreme sul piano coscienziale. Solo Dio è padre, solo Dio è giudice infinitamente giusto, nella sua misericordia inesauribile. Ogni acquisto di potestà terrena, laica o ecclesiastica, è occasione di pericolo, di morte per l'anima. E non basta ancora, non si tratta solo di questo. Allo sguardo inflessibile del romanziere nemmeno la più schietta intenzio- 
ne benefica appare adeguata per assicurare un valore positivo ai nostri atti. Neppure lo spirito di fede più puro garantisce di comportarsi secondo rettitudine: così è anche per Lucia, per Cristoforo, per Federigo. Nessuno è mai al riparo dalle insidie della nostra malfida natura.

Da questo pessimismo angoscioso derivano il criticismo instancabile, l'inquietudine problematica che percorrono il libro investendone uno a uno i personaggi. Qui sta il motivo essenziale per cui nella rappresentazione romanzesca non possiamo non sentirci coinvolti, anche se non ne condividiamo le premesse di fede. La coerenza esigentissima dell'idea di uomo manzoniana, religiosamente fondata, rende impareggiabile l'acume dell'indagine sui vizi e limiti, debolezze e incongruenze dei nostri costumi di vita.

Che poi il narratore abbia voluto soffondere di un sorriso olimpico la spietatezza delle sue analisi psicosociali, costituisce solo la conferma di un fatto: egli non sa e non vuole negare una apertura di credito agli svolgimenti futuri della civiltà borghese, purchè si conformi ai dettami della morale cattolica. E tuttavia, pur mentre lavora a un libro concepito come un atto di fiducia nella possibilità di intervenire positivamente, cioè cristianamente, sull'assetto dell' universo sociale, permane in lui l'assillo di uno scetticismo invincibile. Di nuovo emerge la contraddizione tra la sfiducia profonda nella natura umana e il desiderio struggente di un miglioramento, un progresso delle forme di vita consociata.

Non per nulla l'esperienza del gran romanzo resta senza seguito, quasi che lo scrittore abbia voluto lasciarsi alle spalle i tumulti menzogneri della vita attiva per volgersi alla pace appagante della vita contemplativa. Ma l'ansia di collaborazione al miglioramento dell'esistenza collettiva non si estingue: si trasferisce dal terreno dei componimenti misti di storia e d'invenzione a quello degli studi di lingua, in una prospettiva energicamente socializzante. Scrive Maurizio Vitale, nello 
studio già citato: "Il Manzoni era infatti convinto che l'avvento di una lingua nazionale unitaria avrebbe grandemente contribuito a determinare una elevazione culturale dei ceti più poveri e incolti, costretti alla separazione e alla sudditanza dalla limitata e povera abitudine dialettale, consentendo loro un più facile accrescimento delle cognizioni e un largo incremento delle possibilità espressive."

Parrebbe dunque di trovarsi sul piano di una pragmatica con forte slancio di immediatezza operativa. Ma lo stesso illustre storico della lingua compie un'aggiunta altamente significativa, sostenendo che «l'unità linguistica così caldamente patrocinata pareva certo allo stesso Manzoni, per i tempi, 'una bella utopia”. E più avanti ecco sottolineato che «l'affermazione e accettazione totale in tutta la nazione di un definito tipo linguistico, si presentava - nel progetto democratico e giacobino manzoniano- come fatto inedito, nuovo, di valida operante suggestione».

Manzoni utopista, Manzoni democratico e giacobino, non nella gioventù ma nell'età matura, e sia pure in un àmbito assai dissimile da quello della creatività letteraria: non potrebbe esserci attestazione migliore della vocazione alla militanza di uno scrittore quanto mai alieno dal perbenismo appartato e rassegnato. 



\section{Alfredo Stussi}

\section{Conclusioni}

Le relazioni che abbiamo ascoltato presentano, pur nella loro notevole varietà di temi e di impostazioni, un tratto comune perché sono in rapporto più o meno stretto, ma sempre evidente, con indirizzi di ricerca coltivati da Maurizio Vitale. Dei sei relatori, tre sono storici della lingua italiana, ma di questi solo Silvia Morgana è stata sua allieva diretta, non Francesco Bruni, né Angelo Stella. Tuttavia i tre loro interventi rendono omaggio a Maurizio Vitale collegandosi a settori della ricerca dove egli è stato ed è indiscusso Maestro. Lo dicono chiaramente già titoli come "Ho fato translatare in lingua nostra». Tra Milano e Ticino ducale: lingua cancelleresca e varietà d'uso (Morgana) e Toscani e Lombardi (Bruni); a maggior ragione ha tale caratteristica la sintesi preliminare di Stella (I tempi della lingua, i luoghi degli scrittori) dedicata alle ragioni e ai contenuti della Mostra di stampe e manoscritti direttamente connessi all'attività di ricerca cui Vitale si è dedicato, e si dedica con intatta passione e giovanile energia, da oltre sessant'anni.

Tuttavia ancor di più importa notare che gli altri tre relatori non sono storici della lingua. Mi sembra insomma significativo questo coinvolgimento di 'esterni', che hanno trovato in opere fondamentali di Vitale sulla questione della lingua, su Tasso, sugli esuli religionis causa e su Manzoni, uno stimolo efficace per intraprendere indagini in altre direzioni, per fornire approfondimenti o prospettive complementari.

Grazie dunque a Isabella Gualandri abbiamo riconosciuto nella Gerusalemme liberata ulteriori tracce di classici latini, e precisamente quelle del Bellum civile di Lucano; Susanna Peyronel ci ha mostrato come Alessandro Citolini e Ludo- 
vico Castelvetro fossero perseguitati quali eretici per la loro adesione a idee di rinnovamento spirituale e di riforma religiosa; Vittorio Spinazzola ha ricondotto la discontinuità tra linguaggio narrativo e linguaggio apologetico nei Promessi sposi alle ragioni e ai modi in cui si realizza la politicità del romanzo, cioè l'intento manzoniano di rivolgersi a un pubblico universale.

Gualandri, Peyronel e Spinazzola testimoniano dunque quanto l'opera di Vitale irraggi benefici effetti anche al di là del perimetro più strettamente professionale in cui si colloca; ma mentre si può dare per scontato il coinvolgimento di storici e critici della letteratura italiana, non altrettanto scontato pare quello delle due studiose prima nominate, cultrici, rispettivamente, di filologia classica e di storia moderna. Che ciò succeda è senza dubbio sintomatico del fatto che al centro dell'attività scientifica di Vitale ci sono temi nodali nella storia linguistica italiana, dalla questione della lingua alla lingua dei più grandi scrittori, temi dunque che esercitano una forte attrazione non solo su studiosi di varia specializzazione, ma in genere sulle persone colte.

Però non si tratta solo di questo, e a me pare che conti molto anche l'innata attitudine dialogica della persona. Mi spiego: attivo in una grande Università come la Statale di Milano, Maurizio Vitale ha sempre prestato attenzione a quanto di serio e di importante avviene in settori della ricerca altri dal suo; nello stesso tempo ha saputo cogliere le occasioni di interscambio culturale offertegli da quei crogiuoli di competenze diverse che sono l'Istituto lombardo e l'Accademia dei Lincei. In ciò la curiosità intellettuale e l'apertura mentale dello studioso si accompagnano al prezioso ingrediente della socievolezza, cioè della disponibilità a ricevere e a dare in ugual misura, ma non indiscriminatamente, perché la benevolenza o addirittura l'affetto di Maurizio Vitale si manifestano solo dopo attenta selezione dei destinatari. Altrettanto 
evidente è quella che chiamerei la sua capacità di governo, cioè di individuare con immediata lucidità i termini di un problema, di indicare la strada da percorrere quando altri ancora brancolano nel buio, di rimettere sui binari giusti un dibattito. Il che non vuol dire essere infallibile e tanto meno pretendere di esserlo, ma essere capace di individuare il punto cruciale su cui confrontarsi, senza divagazioni inutili. In ciò si manifesta dunque un'attitudine che con tutta evidenza caratterizza, mutatis mutandis, lo studioso: l'analisi più accurata e minuziosa deve dar luogo a una sintesi che ne dichiari il senso complessivo, le ragioni che l'hanno determinata e guidata, senza indugiare o smarrirsi strada facendo.

Occorre insomma interrogarsi sul 'senso della storia', nella fattispecie della storia linguistica. Questa sorta di imperativo categorico determina la caratteristica complessiva di oltre sessant'anni di attività scientifica impegnata su un fronte larghissimo, ma funzionale, come ha sottolineato Angelo Stella, a una prospettiva ben ferma: quella che individua nella lingua un fattore di coesione del popolo italiano nel suo lento e faticoso percorso verso l'unità nazionale (basterà ricordare, come ha fatto anche il Presidente dell'Istituto Lombardo, il saggio una sorta di manifesto programmatico - intitolato La lingua: fondamento della nazione italiana). Di qui le fondamentali e pionieristiche ricerche sulla cosiddetta questione della lingua e sui grandi scrittori che della nostra lingua hanno determinato importanti caratteristiche, e non solo al livello letterario. Tuttavia l'attenzione alle grandi linee di sviluppo dal punto di vista linguistico e letterario non comporta che siano messi in secondo piano i caratteri specifici dei singoli testi, compreso l'iter spesso tormentato della loro genesi.

Spicca da ultimo la monografia dedicata all'Orlando furioso dove la serrata descrizione della lingua della prima redazione ( 5 I6) consente a Vitale, confrontandola con le "correzioni» successivamente apportate, di fornire una sostanziosa e ori- 
ginale messa a punto in merito alla forma linguistica assunta dal poema nell'edizione definitiva del I 532 . Ma prima di Ariosto, questo tipo di analisi, che fa tesoro delle correzioni, era stato riservato a Petrarca, Boccaccio, Leopardi, Manzoni (e forse qualcosa dimentico), dando luogo a saggi che - ricordo quello dedicato a I criteri della prassi correttoria manzoniana - valgono come modelli da proporre a chi volesse intraprendere analoghe indagini su altri scrittori. Nell'utilizzo delle varianti d'autore Vitale occupa dunque una posizione di primo piano e per il numero e per l'importanza delle ricerche effettuate. Ricerche nelle quali si manifesta quella sensibilità e quel gusto per il testo letterario che altrove viene direi quasi represso, come se potesse turbare il ferreo rigore con cui sono condotte analisi linguistiche che non lasciano niente di inesplorato, niente che non sia interpretato grazie a una perfetta padronanza dei più efficaci strumenti di indagine.

Col che si viene a dire che Vitale è uno storico della lingua completo, di un tipo cioè ormai raro e forse addirittura scomparso, perché ha studiato la lingua come fenomeno in largo senso culturale, e in pari misura la lingua nelle sue caratteristiche intrinseche, cioè fonetiche, morfologiche, sintattiche. Lo ha fatto precocemente, dando alle stampe nel 1953 La lingua volgare della Cancelleria Visconteo-sforzesca nel Quattrocento e nel 1960 La questione della lingua, libri che per molti di noi, allora apprendisti, hanno contato non poco.

Sempre il suo è stato un impegno sul piano scientifico accompagnato da esemplare onestà intellettuale: infatti Vitale non rinuncia mai ad andare a fondo dei problemi anche quando sono di scoraggiante difficoltà e magari potrebbero essere accantonati con un'accorta dissimulazione; insomma non risparmia tempo ed energie per l'accertamento scrupoloso dei fatti, inteso quale indispensabile base di ogni interpretazione. Anche per questo è un Maestro da additare come modello alle giovani generazioni di studiosi. 


\section{Abstracts}

\section{SIRONI}

L'Istituto, cui il ministro napoleonico Lugi Vaccari conferiva duecento anni fa l'incarico di costruire un nuovo vocabolario della lingua italiana, e che con l'opera dei suoi grandi maestri - Vincenzo Monti, Simone Stratico, Giovanni Gherardini, Alessandro Manzoni, Graziadio Isaia Ascoli, Carlo Salvioni - ha risposto assiduamente e genialmente a quella sollecitazione, in questo "Incontro di studi» rende onore a Maurizio Vitale, suo decano, per quattro decenni docente di Storia della lingua italiana presso l'Università degli Studi di Milano, Maestro della disciplina, in una ricerca sempre viva e continua. Illuminante premessa e prospettica cornice agli interventi di scolari e di amici, che seguono, restano le pagine da lui lette all'Accademia dei Lincei su La lingua italiana e l'unità nazionale, che stimolano, soprattutto oggi, al recupero e al rinnovamento della nostra identità civile e culturale nella condivisione della casa comune che è la lingua nostra.

The Institute, which was invited two hundred years ago by Luigi Vaccari, minister in Napoleon's Italic Kingdom (Regno Italico), to compile a new dictionary of the Italian language and which, through the work of its greatest masters - Vincenzo Monti, Simone Stratico, Giovanni Gherardini, Alessandro Manzoni, Graziadio Isaia Ascoli, and Carlo Salvioni to name the most famous - responded diligently and imaginatively to the invitation, organized a study day in honour of its dean, Maurizio Vitale, professor of History of the Italian Language at the University of Milan for four decades and undisputed master of the discipline, with the presentation of outstanding current research. Vitale's lecture La lingua italiana e l'unità nazionale, which he read to the Accademia dei Lincei, provides an eloquent ntroduction and backdrop to these essays by his students and friends, encouraging us, in these days especially, to rediscover and renew our civic and cultural identity as we inhabit the many mansions that go to make up the Italian language.

\section{STELla}

Gli scritti che Maurizio Vitale ha proposto nell'arco di oltre sessant'anni si organizzano nella riflessione degli studiosi della storia della lingua italiana, e non solo, come monografie, capitali, paragrafi di una summa che della «lingua nostra» ricostruisce interpreta giudica le vicende dalle Origini al 
presente. In questa prospettiva, tenendo conto della prassi metodologica e del rigore culturale, si riprendono qui alcune osservazioni stimolate da tre suoi contributi sottovoce, quelli dedicati alle varianti d'autore nel Decameron, al poema dell' 'omerida» Trissino, L'Italia liberata da' Gotthi, alla "saga molinaresca» del dimenticato, da altri, Bacchelli. L'esattezza numerica degli spogli grammaticali, lessicali, retorici e il loro complementare intrecciarsi in strutture pluriespressive, conducono e sospingono, con invitante forza valutativa ed assertiva, oltre la soglia della lingua, alla forma stilistica dello scrittore.

In the panorama of reflection by historians of the Italian language (and not only), Maurizio Vitale's writings, spanning a period of more than sixty years, function in a way not dissimilar to the subdivisions of a summa, reconstructing, interpreting and assessing the various developments in the language from its origins to the present. This article seeks to do justice to Vitale's rigorous methodological culture and practice by focusing on three of his less well-known works in particular: his essay on the authorial variants of Boccaccio's Decameron, his monograph on the narrative poem L'Italia liberata da' Gotthi by the slavish Homeric imitator Trissino, and his work on the Il Mulino del Po trilogy by the now largely forgotten (but not by Vitale) Riccardo Bacchelli. The mathematical precision of Vitale's analysis of these authors' grammar, lexis and rhetoric, and the force and persuasiveness with which he shows how individual items are weaved into expressive structures, invite the reader to look beyond the confines of the writers' language, to consider questions of their style.

\section{Gualandri}

Benché nei suoi trattati sul poema eroico il Tasso più di una volta critichi diversi aspetti del poema di Lucano, tuttavia il suo influsso traspare in molti passi della Gerusalemme liberata, sia per la struttura di vari episodi, sia per reminiscenze formali. Il contributo, concentrandosi su alcuni passi, cerca di mettere in luce come la poesia di Lucano, audace e possente e spesso considerata manieristica o barocca, potesse essere in consonanza col gusto del Tasso.

Although Tasso is critical of aspects of Lucan's poem on several occasions in his discourses, the latter's influence is nonetheless visible in various passages of the Gerusalemme liberata, in terms of the structure of several episodes and also formal reminiscences. This paper focuses on several such assages, seeking to demonstrate how the poetry of Lucan, bold and daring in many ways rather than manneristic and Baroque as it is often held to be, was in fact very much in keeping with Tasso's own aesthetic. 


\section{Peyronel}

Tra i molti protagonisti del dibattito sulla lingua, che animò la scena culturale italiana del primo Cinquecento, furono alcuni intellettuali e letterati interessati al dibattito religioso di quei decenni e alle proposte di riforma, o di rinnovamento spirituale, che maturarono anche in Italia. Alcuni di questi letterati, come Alessandro Citolini o il più famoso Ludovico Castelvetro, furono oggetto di sospetti, accuse e processi da parte dell'Inquisizione, e divennero esuli religionis causa. Lo slancio divulgativo culturale, che caratterizza i primi decenni del Cinquecento, si intrecciò con la questione della lingua e con la volgarizzazione della cultura, anche religiosa ed eterodossa. Questi letterati, nei loro scritti sulla lingua, introdussero pensieri ed osservazioni che, in modo nicodemitico, alludevano anche alle proprie pericolose propensioni religiose. Costretti alla fuga, portarono fuori d'Italia tutta la passione per quello splendido volgare ormai maturo per trattare ogni materia, per allargarsi a ogni disciplina e per conquistare anche l'Europa.

Some of the leading protagonists of the questione della lingua which dominated the Italian cultural arena in the early Cinquecento were intellectuals and men of letters involved in the religious debate of those decades, and in the ideas of reformation - or spiritual renewal - developing in Italy at the time as well as the rest of Europe. Some of these men, such as Alessandro Citolini or the better-known Ludovico Castelvetro, were the target of suspicions, accusations and trials on the part of the Holy Inquisition, and elected to go into exile on religious grounds as a result. In this way the spread of Italian culture which was such a feature of the early decades of the sixteenth century took place in conjunction with the linguistic debates and the translation of non-conformist religious texts. These men of letters often introduced thoughts and observations in their linguistic writings which alluded cryptically to their dangerous religious tendencies. Forced to flee, they took with them their passion for the glorious Italian vernacular, now mature as vehicle for even the noblest of subjects, helping it progress beyond the confines of Italy its conquest of all disciplines as well as the rest of Europe.

\section{MorganA}

Alcuni fondamentali saggi di Maurizio Vitale documentano l'evoluzione della koinè in uso nella Cancelleria del Ducato di Milano nel corso del secolo XV, e la sua graduale convergenza verso il modello fiorentino. Questo saggio analizza la situazione linguistica delle aree svizzere di confine, al tempo parte integrante del dominio visconteo-sforzesco, riferendosi al cospicuo corpus delle lettere inviate agli uffici amministrativi milanesi dai funzionari ducali, come pure ai testi statutari, ad altre testimonianze 
documentali, a scritti di comuni cittadini, residenti o emigrati. La ricerca evidenzia che la koinè progressivamente si impone e viene accettata non solo nella scripta ufficiale, ma anche nell' uso quotidiano e privato. Si rileva dunque come caratteristico del Ticino, già nel Medioevo, un mistilinguismo determinato da una situazione geopolitica, propria della «Lombardia elvetica», terra di confine e di transito, tra il mondo germanico e la Lombardia 'italiana'.

A series of seminal articles by Maurizio Vitale documents the development in the koine used in the Duchy of Milan Chancellerry in the course of the fifteenth century, showing how it gradually converged towards the Florentine model. This essay analyses the linguistic situation in the Swiss border regions, which at the time were an integral part of the Visconti-Sforza dominion, focusing in particular on the substantial corpus of letters sent by the Duchy's officers to the Milanese administrative offices, the texts of statutes and other documentary testimonies and writings by common citizens, both residents and émigrés. The research shows that the koinè gradually became established and was accepted not only in the official scripta, but also in daily and private use. This kind of hybrid linguistic situation emerges as typical of the Ticino region from as early as the Middle Ages, due largely to the geopolitical situation of "Swiss Lombardy" as a region of border and transit between the Germanspeaking world and its Italian counterpart.

\section{BRUNI}

Punti di partenza del lavoro sono l'opera di Maurizio Vitale sulla questione della lingua e il suo contributo Lombardi e toscani nella questione del Vocabolario, che analizza il difficile rapporto in materia di vocabolario della lingua italiana tra l'Istituto di Milano e l'Accademia della Crusca di Firenze, negli ultimi anni del dominio napoleonico e nei primi della restaurazione austriaca. Il dualismo tra toscani e lombardi in fatto di lingua consiste nella coscienza di un tetto linguistico comune e nello stesso tempo nel rifiuto, da parte del centro culturale milanese, del primato linguistico comunque rivendicato da Firenze. La controversia grammaticale, lessicografica e anche filologica Milano-Firenze accompagna la storia culturale italiana, e affiora più volte nella traiettoria dalle origini medievali al Proemio (1872) di Ascoli, con le specifiche connotazioni di luoghi, di tempi, di circostanze, di autori. Da qui la rilettura critica di episodi variamente esaminati: il Serventese lombardesco, alcuni luoghi di Dante, Il Negromante dell'Ariosto, Sabba da Castiglione, il Bandello, un articolo di Alessandro Verri nel "Caffè», la collezione milanese dei "Classici Italiani», e infine la concezione linguistica di Manzoni a fronte della polemica antimanzoniana di Ascoli. 
This essay discusses Maurizio Vitale's work on the questione della lingua, in particular his article Lombardi e toscani nella questione del Vocabolario which analyses the difficult relationship between the Milanese Istituto and the Florentine Accademia della Crusca on the issue of the Italian language dictionary promoted by the Napoleonic kingdom in its final years prior to the Habsburg restoration. The Tuscan-Lombard rivalry arises from the fact that, despite an awareness on both sides of sharing the same language, the Milan cultural centre has always refused to acknowledge the linguistic primacy which Florence insists on claiming. The Milan-Florence dispute, played out on grammatical, lexicographical and philological levels, has been a constant refrain throughout Italian cultural history, flaring up on several occasions from its medieval origins to Graziadio Ascoli's Proemio in 1872, in various forms and means according to the specific places, times, circumstances and authors concerned. This scenario forms the context for a series of critical reexaminations: the Serventese lombardesco, several passages of Dante, Ariosto's Il Negromante, Sabba da Castiglione, Bandello, an article by Alessandro Verri published in "Il Caffè», the Milanese collection of "Classici Italiani», and, finally, Manzoni's understanding of language in view of Ascoli's antiManzonian polemics.

\section{SPINAZZOLA}

La politicità del capolavoro manzoniano si fonda sul proposito dello scrittore di porre il suo ingegno letterario al servizio di una impresa di riconquista cattolica del mondo moderno. Manzoni sceglie la forma del romanzo storico perché l'intreccio fra grandi eventi pubblici e modesti fatti d'invenzione lo porta a metter a fuoco meglio il rapporto fra i diritti di libertà del cittadino, anzitutto lo jus nubendi, e i doveri di responsabilità verso il prossimo, custoditi dalla Chiesa.

The political dimension of Manzoni's masterpiece rests on the author's decision to put his literary creativity at the service of a Catholic initiative to reconquer the modern world. Manzoni chose the historical novel, because in it, the interaction between major public events and the humbler fictional episodes enabled him to highlight more effectively the relationship between the individual citizen's right to liberty (ius nubendi in particular) and the duty of care towards one's neighbour, under the responsibility primarily of the church.

\section{STUSSI}

Le relazioni presentate in questa sede hanno una evidente correlazione con gli svariati temi alla cui discussione Vitale ha offerto un contributo duraturo. Di particolare interesse è il modo in cui studiosi, attivi in ricerche in campi diversi dalla storia della lingua italiana, hanno trovato spun- 
to nei suoi scritti, a conferma che le tecniche e le metodologie sviluppate da Vitale hanno dettato una lettura della storia della lingua e della letteratura italiana come parte essenziale della storia d'Italia nel suo insieme. Il lavoro di Vitale, studioso e docente, rappresenta un esemplare modello per tutti i giovani ricercatori.

The papers presented here have an obvious connection with various themes to the discussion of which Maurizio Vitale has made a long-lasting contribution. Particularly interesting is the way in which scholars working in fields other than the history of the Italian language have taken stimulus from his writings, confirming that Vitale's techniques and methodologies promote a view of the history of language and literature as part of the broader history of Italy. Vitale's work as both scholar and teacher thus constitutes a relevant model for young researchers in general.

Versione inglese di David Gibbons 


\section{INDICE DELLE PERSONE a cura di Giovanni Battista Boccardo}

Achillini, Giovanni Filoteo I 48 e n, I 5 on

Agricola, Georg 88 e n, 89, 90n, 96

Agricola, Rodolphus 97

Alamanni, Luigi 28

Alberti, Leandro I 47-I 50, I 53

Alberti, Leon Battista 27

Alberto Magno, santo 83

Aldobrandini, Pietro 45

Alessio, Gian Carlo I $32 \mathrm{n}$

Alfieri, Vittorio 93n

Alfonso III d'Avalos I 55

Algarotti, Francesco 28, I72

Alighieri, Dante I6, I 8, I9, 27-

29, 42, 88, I03, I 3 In, I36-I 38 ,

I 47, I 48, I 50, I75

Amaseo, Romolo Quirino 76

Ambrosini, Federica 8on, 82n

Angiolieri, Cecco 36

Antonini, Anna 77

Aretino, Pietro 70, I 5 I e n

Ariosto, Ludovico I6, 27, 5on,

54n, I 38, I 39, I 50, I 5 I, I 88

Aristotele 30, 48 e n, 78, 83, 98,

I 45

Arriano 29

Arrighi, Ludovico 26

Ascoli, Graziadio Isaia 6, I I, I 24, I 25, I 28-I3I, I67, I69-I7I

Asor Rosa, Alberto I 2 I

Aubert, Albert 69n

Bacchelli, Riccardo I4, I6, 35 ,

$36,38,40$

Badoer, Federico 82

Baï, Lazare de 97
Bandello, Giacomo Francesco I 53

Bandello, Matteo I 5 I-I 56, I63n

Barbaro, Daniele 80

Bàrberi Squarotti, Giovanni I 40n

Barbieri, Andrea 97 e $n$

Barnaba da Reggio I 33, I 34

Bartesaghi, Paolo I03n, I04n

Basile, Bruno 6In

Bauer, Georg vd Agricola, Georg

Belligni, Eleonora 74n

Bellomo, Leonardo I $58 \mathrm{n}$

Bembo, Pietro 27, 70-72, 75, 88, 90n, 93, I39, I4I, I42n, I45,

I 48 , I 50

Berengo, Marino I6on, I6 In

Bernardino da Siena, santo I I 8

e $n$

Berni, Francesco 28

Besana, Antonio I I 3, I I 8n, I I 9

Bianchi, Patricia I 24n

Bianconi, Sandro Io8n, I I 4n,

I I 8 n, I 20n, I 2 In

Biffi, Giovanni Ambrogio I 5

Boccaccio, Giovanni I6, I7, 2 I,

77, 79, 88, I03, I37, I3 8 e n,

I 42n, I 47, I 48, I 50, I 5 5, I 88

Bocchi, Achille I 48

Boezio, Severino 78

Bologna, Corrado 75n

Bolzoni, Lina $74 \mathrm{n}$

Bongrani, Paolo I07n, I08n

Bonomi, Ilaria 6

Bordoni, Bruno i I 5 n, i i $7 n$

Borghini, Vincenzo 79, I42n

Borranini, Niccolò I 20-I 2 I

Borromeo, Federico I 8o, I 82

Borsa, Giovanni Angelo 
Branca, Vittore I6, I7, 23, I38n

Branda, Paolo Onofrio I03n

Brioschi, Franco I $32 \mathrm{n}$

Brocardo, Iacopo 80

Brocchi, Antonio I I 2

Broggini, Romano I I 4n, I I 5 n

Broglio, Emilio I67, I68 e n, I70

Brown, Josh I06n

Brucioli, Antonio 70

Brunetto, Orazio 8o

Bruni, Francesco I7, 8 In, I04n, IO8n, I I 5 n, I 2 In, I 24n, I33n,

I $72 \mathrm{n}, \mathrm{I} 85$

Budé, Guillaume 75, 97

Bujanda, Jesús Martinez de $84 \mathrm{n}$

Buonarroti, Michelangelo,

il Giovane I 5

Butzer, Martin 74, 96, 97

Calvino, Giovanni 95

Calzona, Arturo 8 In

Camillo, Giulio vd Delminio

Camorana, Francesco 93n

Canetti, Elias 7

Canone, Eugenio I 57n

Carafa, Gian Pietro 75

Caravale, Giorgio 73n

Cardona, Giorgio Raimondo

I 2 In

Carducci, Giosuè 4I 42

Carlo Magno I 32

Carlo v d'Asburgo 29

Carnesecchi, Pietro 72

Caro, Annibale 76, 90

Cartago, Gabriella 6

Casa, Giovanni della I 58

Casella, Angela I39n

Castagna, Franchino I I 4

Castellani, Arrigo 20

Castelvecchi, Alberto I $38 \mathrm{n}$

Castelvetro, Ludovico 48, 67,
90-99, I 86

Castiglione, Baldassarre 27, I 43,

I 46 e n, I 5O, I 53, I74n

Castiglione, Bartolomeo I 44

Castiglione, Sabba I40-I 47, I49,

I 50, I 53

Cattaneo, Carlo I 3,29

Celsi, Mino 72

Cesare, Gaio Giulio 47, 49-5 I,

$53-57,62-64,78$

Cesari, Antonio I 2, I 5, I 65

Cesarotti, Melchiorre 172

Chiabrera, Gabriello 28

Chiari, Alberto I 67n

Chiecchi, Giuseppe 8on

Chiesa, Mario I 4on

Chiesi, Giuseppe Io8n, I Ion,

I I $8 \mathrm{n}$

Cicerone, Marco Tullio 47, 78,

83, 93n

Citolini, Alessandro 67, 72, 74-

$87,93,94,97,99,185$

Claricio, Girolamo I 48

Clemente viI, papa 28, 72

Colli, Barbara I66n

Colombo, Michele ro8n

Colonna, Vittoria 72

Coltellini, Marco 28

Contarini, Gasparo 70, 72, 76, 92

Conte, Alberto I $36 \mathrm{n}, \mathrm{I} 42 \mathrm{n}$

Contile, Luca 76

Cornazano, Antonio I39n

Corsi, Giovanni I7I, I74

Corti, Maria I 2

Cranmer, Thomas 86

Crescimbeni, Giovan Mario I 58 , I6I

Cristiano di Magonza I 33

Crivellucci, Amedeo I 3 In

D'Achille, Paolo I 2 in 
Dalmas, Davide 9in, 95 n

Danieli, Gian Antonio 6

Danzi, Luca I67n

Dardano, Maurizio I I 3 n

Datini, Francesco ro5

Della Giustina, Lisa 83n

Della Valle, Alfonso, marchese di

Casanova 126

Delminio, Giulio Camillo 74,

75,83

De Maldé, Vania $54 \mathrm{n}$

Demetrio Falereo 64

Di Girolamo, Costanzo I $32 \mathrm{n}$

Dionisotti, Carlo 26, 68, 72,

I39n, I42n

Dioscoride, Pedanio 83, 84

Domenichi, Ludovico 80

Donini, Pierluigi 48 e n

D’Ovidio, Francesco I 23-I 3 I

Du Bellay, Guillaume 75

Edoardo vi Tudor 85, 86

Elisabetta I d'Inghilterra 83, 85

Ennio, Quinto 47n

Enrico di Crissey I 32

Enselmino da Montebelluna I 7

Erasmo da Rotterdam 68-7I, 84,

95, 97

Ercole II d'Este 9I

Ernst, Germana I $57 n$

Esichio 96n

Ezzelino III da Romano I 36

Fallopia, Giovanni 93n

Fanfani, Pietro I7

Farnese, Pier Luigi 28

Federico III d'Asburgo I I I

Felici, Lucia 9 In, 93n

Fenzi, Enrico I $38 \mathrm{n}$

Ferrario, Giulio I 60

Fessia, Lina 86n
Filicaia, Vincenzo da 28

Fiorato, Adelin Charles I $55 \mathrm{n}$

Fiordibello, Antonio 93n

Fiore, Francesco Paolo 8 in

Fiorelli, Piero I64n

Firpo, Luigi $67 n, 87 n, 88 n$, 9on

Firpo, Massimo 67n, 68n, 69n,

75n, 76n, 8o e n

Flaminio, Marcantonio 75, 76

Flora, Francesco i 5 in

Florido, Francesco 76, 77

Florio, Michelangelo 86, 87, 89,

90

Fochessati, Ada 36

Folena, Gianfranco I2, I40n,

I6on

Folengo, Teofilo I 4 on

Formisano, Luciano I $38 n$

Forteguerri, Niccolò 28

Fortunio, Giovanni Francesco 7I, 88, I 48

Foscolo, Ugo I72-I 74

Fragnito, Gigliola 75n

Francesco d'Assisi, santo 73

Francesco I di Valois 74

Francesco i Sforza I I on, I I 8n

Francioni, Gianni I $58 \mathrm{n}$

Franco, Niccolò 68-70

Frangioni, Luciana I05n

Frasso, Giuseppe 6, I05n

Fregoso, Cesare I 55

Froben, Johann 88, 89

Fubini, Mario I73n

Füssli, Johann Heinrich 173

Gabrieli, Vittorio 85n

Gadda, Carlo Emilio I6, 25

Galeazzo Maria Sforza I I o

Galeno 83

Gamba, Bartolomeo I6In, I62

e $n$ 
Garavelli, Enrico 93n

Gavazzeni, Franco I74n

Gentilini, Anna Rosa I40n

Geri, Lorenzo 96n

Gherardini, Giovanni 6

Gheri, Goro I42n

Ghinassi, Ghino I67n

Ghisalberti, Fausto I67n

Giacomo da Trezzo I I 9

Giacone, Franco 75n

Gian Galeazzo Maria Sforza Io6

Gianicolo, Tolomeo 25, 26

Gigliucci, Roberto 63n, 94n, 95n

Gilardoni, Virgilio I I 8n

Giorgini, Giovan Battista I67,

I69, I70

Giovan Battista di Biasca I 20

Giunta, Bernardo I39n

Giunti, Filippo I42n

Giunti, Iacopo I 42n

Giusti, Innocenzo Domenico I60

Godi, Carlo I 53n

Goldoni, Carlo I6on, I63n

Gonzaga, Scipione 45, 5 I

Grassi, Corrado I69n

Gray, Henry, duca di Suffolk 87

Gray, Jane 87

Gregori, Mina I I 8n

Griselini, Francesco I I

Grossi, Tommaso 4I

Gualandri, Isabella I 85, I 86

Gualteruzzi, Carlo I42n

Guarini, Battista 28

Guglielminetti, Marziano son

Guglielmo di Tiro 50, 5 I

Guicciardini, Francesco 7, I7 I

Guido da Pisa I 36 e n

Hatton, Cristopher 85, 86

Holtus, Gerhard I08n

Jansen, Dirk Jacob 98n

Jossa, Stefano 94n

Josse de Marvilla, Johannes I 32

Isella, Dante I66n

Italia, Paola I66n

Kürschener vd Pellicanus

Lachin, Giosuè I 35n

Lamberti, Luigi I I

Lando, Ortensio I 57

Lautizio Perugino vd Rotelli

Lavezzi, Gianfranca I $74 n$

Lefèvre d'Étaples, Jacques 75

Le Goff, Jacques I $32 \mathrm{n}$

Leigh, Matthew 56n

Lentulo, Scipione 89 e $n$

Leone $\mathrm{X}$, papa 28 , I 38

Limentani, Alberto I $37 \mathrm{n}$

Linacre, Thomas 97

Lippi, Lorenzo I 5

Livio, Tito I 8, 78, I7I, I72

Leopardi, Giacomo I6, I 88

Lombardi, Elena $\mathrm{I} 74 \mathrm{n}$

Loporcaro, Michele I I $5 \mathrm{n}$

Lotto, Lorenzo 80

Louthan, Howard 98n

Lucano, Marco Anneo 45-50,

$52-65$, I 85

Ludovico di Savoia I 20

Ludovico Sforza, detto il Moro

Io6

Lunier I I

Luparia, Paolo I 4on

Lurati, Ottavio Io8n

Lutero, Martin 7 I, 73

Luzzatto, Leone I 3 In

Machiavelli, Niccolò 29, I39n

Maconi, Ludovica I67n 
Maffei, Lamberto 6

Maier, Bruno 52n, I43n

Malato, Enrico I $38 \mathrm{n}$

Manuzio, Aldo 7 I, 83

Manuzio, Paolo 83

Manzoni, Alessandro 6, I I, I3,

I6, I 8, 24, 29, 36, 40-42, I 24-

I 26, I 28, I30, I66-I69, I75,

I77-I 8 I, I 83, I 85, I 88

Maraschio, Nicoletta I07n

Marazzini, Claudio 67n, 9on, 94

e n, I 57n, I64n, I67n

Margherita d'Angoulême 74, 75

Maria I Tudor 87

Marino, Giovan Battista 53, 54n,

65, 94

Masi, Giovanni Tommaso 27

Masoero, Mariarosa I 4on

Massimiliano II d'Asburgo 98

Mattioli, Emilio 69n, 7on

Mattioli, Raffaele I 4

Mazzacurati, Giancarlo 9in

Mazzali, Ettore 45n

Mazzoni, Guido $72 \mathrm{n}$

Medici, Ippolito de' 72

Medici, Lorenzo de' 106

Melano, Alessandro 93n

Melantone, Filippo 74, 96, 97

Melzi d'Eril, Francesco I 60

Meneghello, Luigi I7

Meneghetti, Maria Luisa I 35n

Metzeltin, Michael Io8n

Migliorini, Bruno I 2, I33n

Mistral, Frédéric 29

Molo, Giovanni I I 3

Molza, Francesco Maria 76

Mongini, Guido 67n, 96n

Montale, Eugenio 29

Monti, Vincenzo 6, I I, I 29, I65, I 66, I72

Montuori, Francesco I 38 n
Mora, Gian Giacomo I 3

Morandi, Luigi I 26-I 30

Mordenti, Rosa 8on

Morgana, Silvia 6, I03n-I05n,

I07n, I08n, I85

Moroni Stampa, Luciano i ion

Mortara Garavelli, Bice I I 3 n

Motolese, Matteo 94n, 97n

Mozzarelli, Cesare 98n

Münster, Sebastian 97

Muratori, Ludovico Antonio 93

e $\mathrm{n}$

Mussafia, Adolfo I7

Napoleone I Bonaparte I6I

Naselli, Carmelina 86n

Nencioni, Giovanni I 2, I36n

Nevio, Gneo 47n

Niccoli, Ottavia 69n

Ochino, Bernardino 73, 85, 86

Omero 28, 29, 31, 32, 46

Orazio Flacco, Quinto 45, 49, 57

Orelli, Giorgio 3 I

Ortolani, Giuseppe I6on

Ostinelli, Paolo Io8n, I I 8n

Ottone I di Sassonia I 54

Overell, Ann 86n

Ovidio Nasone, Publio 45

Palladio, Andrea 35

Paolo, santo 72, 73, 95

Paolo III, papa 20, 67, 72, 75

Paolo Iv, papa vd Carafa, Gian

Pietro

Paradisi, Giovanni I I

Paratore, Ettore 50 e n, 5 I e n, $53 \mathrm{n}$

Parini, Giuseppe I6, I03 e n, I04

e n, I06 e n, I 59, I72

Parmenide 83 
Pascoli, Giovanni 42

Paselli, Giorgio I 3

Paselli, Pasello I I 3

Pasolini, Pier Paolo I6

Pasquali, Giambattista I6on

Pastore, Alessandro 76n

Partenio Tosco I 5

Pellegrini, Giuliano 87n

Pellicanus, Konrad 96

Pellizzari, Patrizia I 4on

Pelo, Adriana I I 3 n

Pescia, Lucrezia I I $5 \mathrm{n}$

Petrarca, Francesco I6, I7, 24,

$25,27-30,72,77,88,95, \mathrm{IO} 3$,

I05, I $38 \mathrm{n}, \mathrm{I} 42 \mathrm{n}, \mathrm{I} 47$, I 50, I 5 I,

I 56, I 88

Petronio Arbitro 48n

Peyronel Rambaldi, Susanna 73n, I 85 , I 86

Piccolomini, Alessandro I 5 in

Pierno, Franco 89n

Pignatti, Franco 55n, 69n

Pindemonte, Ippolito 172

Pipino re d'Italia 132

Pisapia, Giuliano 6

Platone 83

Plinio Cecilio Secondo, Gaio 83

Pole, Reginald 70, 72

Poliziano, Angelo 97

Pometta, Giuseppe Io9n, I I 8n

Pompeo, Gneo 47, 49, 53-55,

59-6I, 63, 64n

Pompeo, Sesto 47

Pomponazzi, Pietro 8I, I45

Ponza, Michele 4I

Pozzi, Giovanni I $57 \mathrm{n}$

Pozzi, Mario I 40 n

Procopio di Cesarea 29

Prosperi, Adriano 75n, 79n

Pulci, Luigi 28

Pusterla, Branda I I I
Quint, David 53n, 57n

Quintiliano, Marco Fabio 49,

$65 \mathrm{n}, 83$

Raboni, Giulia I66n

Raffaele, Ferdinando I33n

Raimondi, Ezio 52 e n, 57n, 64n, 90, 93n, 95n

Rambaldi, Enrico Isacco 6

Rangone, Costanza I 55

Rasori, Giovanni 172

Rinaldi, Michele I36n

Rohlfs, Gerhard I33n

Romagnoli, Sergio I $58 \mathrm{n}$

Romani, Werther 92n

Romano, Ruggiero I32n

Roncaccia, Alberto 9In, 92n, 94n

Ronchi, Gabriella I39n

Rossi, Aldo I7

Rossignoli, Claudia 96n

Rotelli, Lautizio di Meo de' 26

Roussel, Gérard 75

Rovelli, Giovanni I I 8n

Rucellai, Giovanni 25

Rummel, Erika 97n

Rusca, Franchino I Io

Rusca, Pietro I I 2

Ruzante, Angelo Beolco detto

I 45

Sabatini, Francesco 6

Sadoleto, Iacopo 93n

Sadoleto, Paolo 93n

Salimbene de Adam I33, I34 e n

Sallustio Crispo, Gaio 78

Salviati, Leonardo I 5, 80, I62 e

$\mathrm{n}, \mathrm{I} 63$ e $\mathrm{n}$

Salvini, Anton Maria I 5, I 72

Salvioni, Carlo 6, i i, i I 5n, i i 6n

Sannazaro, Iacopo 6I, I 24, I42n

Santagata, Marco I $37 \mathrm{n}$ 
Santini, Emilio I72n

Sauli da Passano, Caterina 80

Sbaragli, Luigi $72 \mathrm{n}$

Scalia, Giuseppe I34n

Scarpati, Claudio I 40n-I43n,

I 46n, I 49n, I 5on

Sceler, Antonio I 20

Schiaffini, Alfredo I 2

Schmitt, Christian ro8n

Secret, François $75 \mathrm{n}$

Segre, Cesare 34, I07n, I36n

Seidel Menchi, Silvana 69n-7 In

Serlio, Sebastiano 8o

Servio Mario Onorato 48, 49n

Severi, Luigi 7 In

Sforza, Ippolita I 55

Silio Italico 45, 47n

Simonetta, Cicco I I 9

Slataper, Scipio 64n

Soldani, Arnaldo $57 \mathrm{n}$

Sordello da Goito I 35n, I 37

Sorella, Antonio 82n

Speroni, Sperone 80-82, I 45

Spinazzola, Vittorio I 86

Stabile, Giorgio 74n

Stazio, Publio Papinio 45, 49,

63n

Stefinlongo, Antonella I I $3 \mathrm{n}$

Stella, Angelo 6, i I 5n-I I 7n,

I $67 \mathrm{n}, \mathrm{I} 85, \mathrm{I} 87$

Sterne, Laurence 173

Stratico, Simone 6, I I

Sturm, Johannes 74

Stussi, Alfredo I 35 e n, I 57n

Tacito, Publio Cornelio 59n

Tansillo, Luigi 28

Tasso, Bernardo I 63

Tasso, Torquato I 6, 25, 28-30, 45 e n, 47-65, I62, I63 e n, I 85

Tassoni, Alessandro 28
Tavoni, Mirko I $37 \mathrm{n}$

Tedeschi, John 86n, 89n, 9on

Tenenti, Alberto $8 \mathrm{In}$

Teodorico I 54, I 56

Teofrasto di Ereso 84, I 46, I74n

Terenzio Afro, Publio 78

Terzoli, Maria Antonietta I73n,

I74n

Tesauro, Emanuele I $57 \mathrm{n}$

Tessa, Delio 36

Tessitore, Fulvio 6

Testa, Enrico I 2 In

Tissoni, Roberto I6on

Tolomei, Claudio 68, 7I-74, 76, 77,85

Tolomeo, Claudio 84

Tomasi, Franco 5on, 53n, 55n,

57n, 63n

Tomasin, Lorenzo I04n

Tomitano, Bernardino 80

Traiano, Marco Ulpio 32

Tremellio, Emanuele 86

Trissino, Gian Giorgio I6, 25-3 I,

34, I 29, I 38 n, I 43 e n, I 48

Troisio, Luciano 8on

Trovato, Paolo I39n, I4In, I46n

Uberti, Farinata degli vd. Farinata

Uberti, Fazio degli ros

Ugolino della Gherardesca 20,

I 36

Ugoni, Giovanni Andrea 80

Vaccari, Luigi 6

Vadiano, Joachim 84 e n

Valentini, Filippo 92, $93 \mathrm{n}$

Valla, Lorenzo 96, 97

Vallone, Raf 39

Varasi, Elena I39n

Varchi, Benedetto 68, 69n, 80,

I7 I 
Varrone, Pietro I09

Vasoli, Cesare 74n, 75n, 8 I e n,

82 e $n$

Vecchio, Paola I I $5 \mathrm{n}$

Vegezio Renato, Flavio 29

Velli, Giuseppe I05n

Verdi, Giuseppe 29

Verdino, Stefano 53n, 55n

Verga, Giovanni 25

Vermigli, Pier Martire 86

Verri, Alessandro I 57-I 59, I6I-

I 63

Vettori, Piero 64

Vico, Giambattista I 6

Vieusseux, Giovan Pietro I64n

Villani, Giovanni I 58

Virgilio Marone, Publio 27, 45, 49 e n, 52, 57, 62, 63 e n, I36,

I 37
Viscardi, Antonio I 2

Visconti, Bruzio I05

Vitale, Maurizio 5-7, I I, I3-I 8, 20, 22-28, 30, 3I, 34-36, 39, 40, $42,43,45,65$ e $n, 67$ e n, 68n, 7I e n, 72n, 76n, 8I e n, 85n, 86n, I04-I07, I23, I30 e n, I3 I e n, I48n, I63, I64n, I74, I75, I 82, I 85 -I 88

Vitruvio Pollione, Marco 35, 76

Vivanti, Corrado I $32 \mathrm{n}$

Watt, Joachim von vd Vadiano

Wyatt, Michael 86n

Yates, Frances Amelia 9on

Zannoni, Giovan Battista I64n Zoppino, Niccolò 7 I 


\section{INDICE DELLE OPERE DI \\ Maurizio Vitale}

Atteggiamento $(L)$ generale di $G$. F. Fortunio in ordine al problema ortografico $\mathrm{I} 2$

Capolavoro (IL) del Boccaccio e due diverse redazioni vd Riscrittura (La) del «Decameron»

Cultura e lingua a Milano nel Trecento I05

Di alcune rivendicazioni secentesche della «eccellenza» dei dialetti i 5

Divagazioni linguistiche dal Trecento al Novecento 4I, 85

"Dizione» (La) formale dell' "Italo Cigno». Notazioni di stile e di lingua nella poesia e nella prosa di Giuseppe Parini I 6 Io4n

Dottrina e lingua di G. F. Achillini teorico della lingua cortigiana I48n

Lingua ( $\mathrm{La}$ ) del Canzoniere (Rerum vulgarium fragmenta) di Francesco Petrarca I6, 26

Lingua (La) della prosa di G. Leopardi: le "Operette morali” I6

Lingua (La) di Alessandro Manzoni I 8, I 30

Lingua (La): fondamento della nazione italiana I 87

Lingua (La) italiana e l'unità nazionale 5, Io4n

Lingua padana e koinè cortigiana nella prima edizione dell'Orlando furioso I 6

Lingua (La) volgare della Cancelleria visconteo-sforzesca nel Quattrocento I 2, IO7n, I 88

Lingua (La) volgare della cancelleria sforzesca nell'età di Ludovico il Moro I $07 \mathrm{n}$

Lombardi e Toscani nella questione del Vocabolario I I, I 3 I, I63, I64n, I74 
Officina (L') linguistica del Tasso epico. La «Gerusalemme liberata»16, 26, 65

Omerida (L') italico: Gian Giorgio Trissino. Appunti sulla lingua dell'«Italia liberata da' Gotthi» 26

Oro (L') nella lingua. Contributi per una storia del tradizionalismo e del purismo italiano 14

Questione (La) della Lingua I 2, I 3, I 6, 26, 67, 68, 7 I, 72n, 76n, 81, I30, I 3 In, I 88

Riscrittura (La) del «Decameron». I mutamenti linguistici I6

Studi di Storia della lingua italiana I 2, I48n

Sul fiume reale. Tradizione e modernità nella lingua del "Mulino del Po» di Riccardo Bacchelli 36

Veneranda (La) Favella. Studi di storia della lingua italiana I I, I4, I 5 , I07n, I3 In 


\section{INDICE GENERALE}

\section{Gianpiero Sironi}

"Abitare LA LINGUA»

PARTE PRIMA

Angelo Stella

I LUOGHI DELLA LINGUA, I TEMPI DEGLI SCRITTORI

Premessa A UN PERCORSO BIBLIOGRAFICO

Isabella Gualandri

IL Tasso e Lucano

DissonanZE E CONSONANZE

Susanna Peyronel Rambaldi

ESULI ITALIANI «RELIGIONIS CAUSA»

E QUESTIONE DELLA LINGUA

PARTE SECONDA

Silvia Morgana

«HO FATO TRANSLATARE IN LINGUA NOSTRA»

Tra Milano e Ticino ducale:

LINGUA CANCELLERESCA E VARIETÀ D'USO

Francesco Bruni

FRA TOSCANI E LOMBARDI

(CON UNA SECONDA SCHEDA SU «FLORENTINITAS»)

Vittorio Spinazzola

LA POLITICITÀ DEI «PROMESSI SPOSI» 
Alfredo Stussi

Conclusioni

I75

$\begin{array}{ll}\text { Abstracts } & \text { I89 }\end{array}$

$\begin{array}{lr}\text { INDICE DELLE PERSONE } & \text { I95 }\end{array}$

INDiCe Delle Opere di Maurizio Vitale 203 
Finito di stampare

da Corrado e Luigi Guardamagna

Tipografi in Varzi

il 7 novembre 2014 
